\title{
Refined computational modeling of SOFCs degradation due to trace impurities in coal syngas
}

\author{
Hayri Sezer
}

Follow this and additional works at: https://researchrepository.wvu.edu/etd

\section{Recommended Citation}

Sezer, Hayri, "Refined computational modeling of SOFCs degradation due to trace impurities in coal syngas" (2014). Graduate Theses, Dissertations, and Problem Reports. 6607.

https://researchrepository.wvu.edu/etd/6607

This Dissertation is protected by copyright and/or related rights. It has been brought to you by the The Research Repository @ WVU with permission from the rights-holder(s). You are free to use this Dissertation in any way that is permitted by the copyright and related rights legislation that applies to your use. For other uses you must obtain permission from the rights-holder(s) directly, unless additional rights are indicated by a Creative Commons license in the record and/ or on the work itself. This Dissertation has been accepted for inclusion in WVU Graduate Theses, Dissertations, and Problem Reports collection by an authorized administrator of The Research Repository @ WVU.

For more information, please contact researchrepository@mail.wvu.edu. 


\title{
REFINED COMPUTATIONAL MODELING OF SOFCS DEGRADATION DUE TO TRACE IMPURITIES IN COAL SYNGAS
}

Hayri Sezer

\author{
Dissertation submitted to the \\ Benjamin M. Statler College of Engineering and Mineral Resources \\ at West Virginia University \\ in partial fulfillment of the requirements \\ for the degree of \\ Doctor of Philosophy \\ in \\ Mechanical Engineering \\ Ismail Celik, Ph.D., Chair \\ Ed Sabolsky, Ph.D. \\ Harry Finklea, Ph.D. \\ Kirk Gerdes, Ph.D. \\ Nianqiang (Nick) Wu, Ph.D.
}

Department of Mechanical and Aerospace Engineering

Morgantown, West Virginia

2014

Keywords: Keywords: Solid Oxide Fuel Cells, Contaminant Degradation, Automatic differentiation, Sensitivity Analysis, Impedance, Polarization, Nickel migration, Electromigration.

Copyright 2014 Hayri Sezer 


\section{ABSTRACT \\ REFINED COMPUTATIONAL MODELING OF SOFCS DEGRADATION DUE TO TRACE IMPURITIES IN COAL SYNGAS}

\section{Hayri Sezer}

The Solid Oxide Fuel Cell (SOFC) is a good alternative for clean and efficient power generation. These cells can be operated directly on a wide variety of fuels including biogas, hydrocarbon fuels and synthesized coal gas (syngas), which is a promising avenue for utilization of coal with much less environmental impact. One of the challenges in this technology is poisoning of SOFC anodes by trace impurities contained in coal syngas. One such impurity, phosphine is known to cause catastrophic failure of SOFC anode even at $<10 \mathrm{ppm}$ concentrations. Fuel impurity degradation patterns can vary by different operating conditions such as humidity, applied current, temperature and anode thickness.

In the present study, more detailed models are developed to predict the typical degradation behaviors observed in SOFC anode due to phosphine by extension of an in-house one-dimensional computational code. This model is first used to predict the effect of steam concentration on phosphine induced degradation in anode supported SOFCs. The model is refined based on the experimental observation, which indicate that the phosphine degradation is less severe in the absence of steam. Simulations results showed good agreement with experimental data. Then, a sensitivity analysis, using dual numbers automatic differentiation (DNAD) is performed to investigate the influence of empirical model parameters on model outputs, electrical potential, ohmic and polarization losses. Further, the refined one-dimensional model is extended to a threedimensional model to study the phosphine induced performance degradation in relatively large planar cells operating on hydrogen fuel. The empirical model parameters are calibrated using button cell experiments and sensitivity analysis as a guide. These parameters are then used in planar cell simulations. The results from the three dimensional model show that the contaminant coverage of nickel and fuel distribution inside the anode is highly non-uniform. These non-uniform distributions are caused by the geometrical alignment of gas channels and current collectors, as well as the variation of gas concentration along the flow direction. The non-uniform deactivation of anode gave rise to the altering of current distribution inside the planar cell such that the cell can still produce current even when some regions of the anode are partially inactive. In addition, to assess the overall cell performance at any given degradation stage, additional simulations are performed to evaluate the electrochemical behavior (polarization and impedance) of the cell. The simulation results are assessed in comparison to experimental observation whenever possible. Finally, a physics based transport model for nickel migration is formulated based on experimentally observed elemental redistribution in a SOFC anode and it is integrated into refined one-dimensional phosphine degradation code. Simulations show that the proposed mechanism of 
Ni diffusion driven by secondary phase formation, the electrical force, and humidity can explain the experimentally observed accumulation of Ni and secondary phases on the SOFC anode surface.

Keywords: Keywords: Solid Oxide Fuel Cells, Contaminant Degradation, Automatic differentiation, Sensitivity Analysis, Impedance, Polarization, Nickel migration, Electromigration. 


\section{ACKNOWLEDGEMENTS}

I wish to express my deepest gratitude to my advisor, Dr. Ismail Celik for his guidance throughout this study. I thanks for all his contributions of time, ideas and funding to make my Ph.D. experience productive. His knowledge, wisdom, experience and hard work have inspired me during these years of my Ph.D. Special thanks to the other members in my graduate committee; Dr. Ed Sabolsky, Dr. Harry Finklea, Dr. Kirk Gerdes and Dr. Nianqiang(Nick) Wu for taking time from their busy schedule to review my work and provide guidance with their questions. I am also grateful to United States Department of Energy (USDOE), National Energy Technology Laboratory (NETL), West Virginia EPSCoR program and West Virginia University for funding this work.

I also thank to the research team members for their friendship and support during my study: Dr. Raju Pakalapati, Dr. Nihan Cayan, Dr. Tao Yang, Dr. Steven Rowan, Albio Guiterrez, Serigo Escobar, Jose Escobar, Sharad Chand, Jerry Hunter Mason, and Satish Guda for good times we had together.

Special thanks to my parents Guley and Seyho Sezer and my sisters, Yildiz, Zeynep, Nuray, my brothers, Salim, Celal, Ihsan, Ulku, Mehmet and Erkan Sezer for their great support, encouragement during my these years of Ph.D. 
This thesis is dedicated

to the memory of my mother, Guley and to my father, Seyho Sezer 


\section{TABLE OF CONTENTS}

LIST OF FIGURES viii

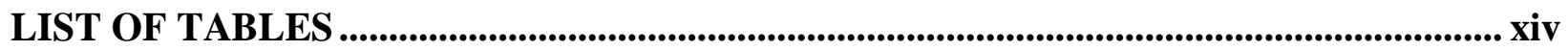

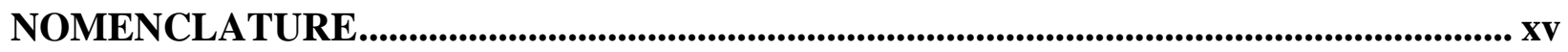

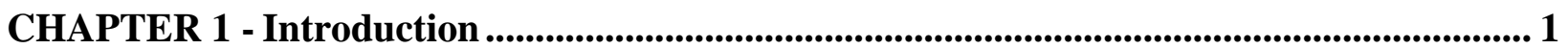

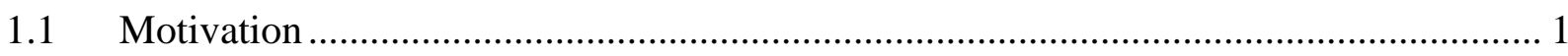

1.2 The Solid Oxide Fuel Cell (SOFC) ….................................................................. 4

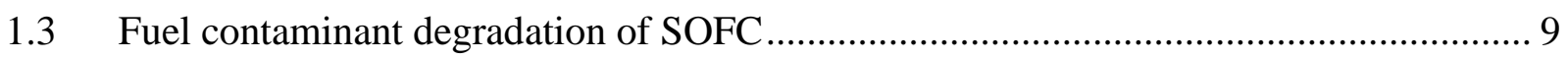

CHAPTER 2 - Literature review, background and objectives............................................ 15

2.1 One dimensional degradation model and polarization, impedance analysis................ 15

2.2 Sensitivity analysis of the model parameters .................................................... 18

2.3 Extension to three dimensional planar cell ......................................................... 20

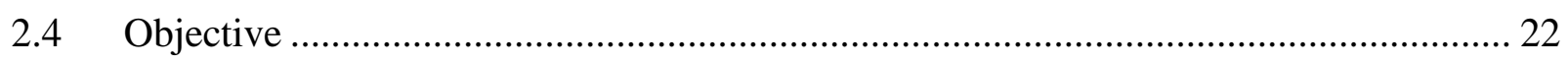

CHAPTER 3 - One dimensional model and polarization, impedance analysis ..................... 23

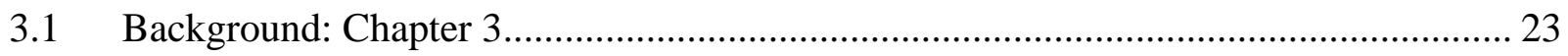

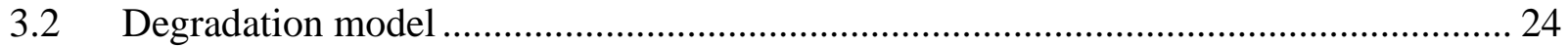

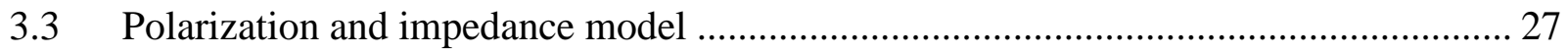

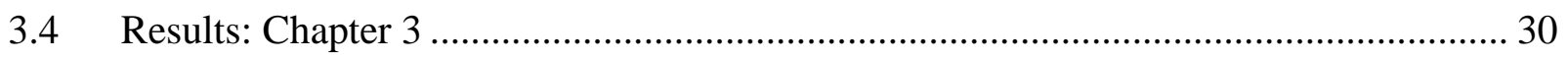

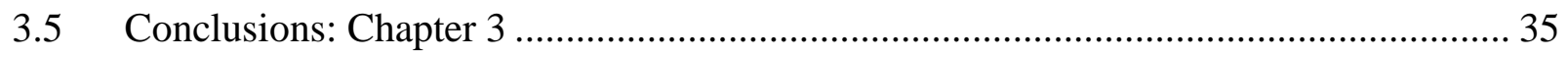

CHAPTER 4 - Sensitivity analysis of the model parameters ................................................... 37 


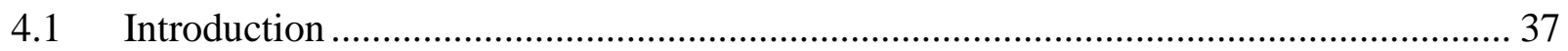

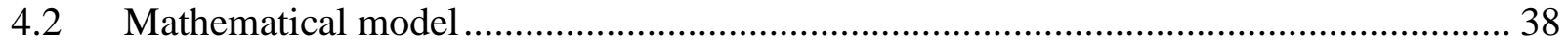

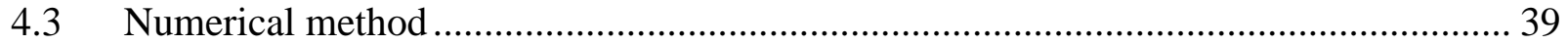

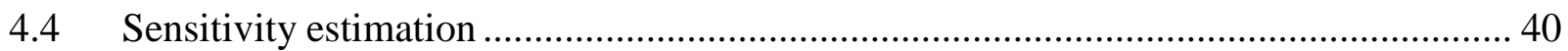

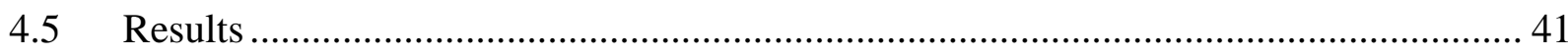

4.5.1 Application to contaminant performance degradation model........................... 45

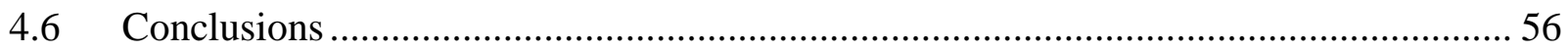

CHAPTER 5 - Extension of the degradation model to three-dimensional planar cell......... 57

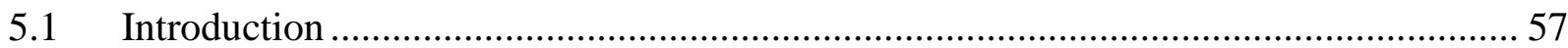

5.2 Validation of the degradation model using PNNL experiments ................................ 61

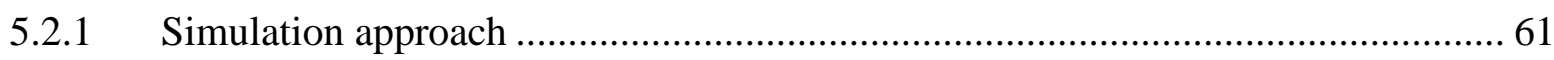

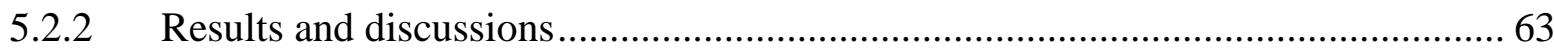

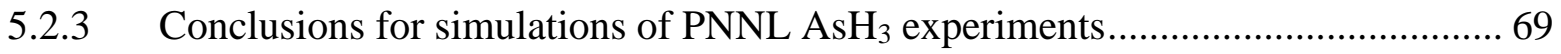

5.3 Electrochemical evaluation of a planar SOFC under phosphine induced degradation .. 70

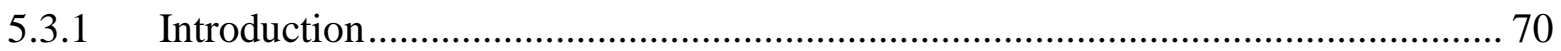

5.4 Planar SOFC computational domain and operation conditions ................................ 71

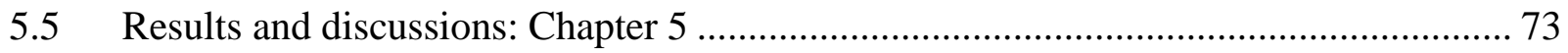

5.5.1 Simulations with ten fuel and ten air channels ........................................... 73

5.5.2 Simulations with one air and one fuel channel ............................................ 78

5.5.3 Electrochemical evaluation of the degraded cell ........................................... 86

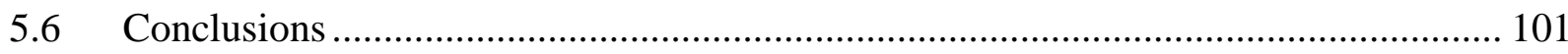

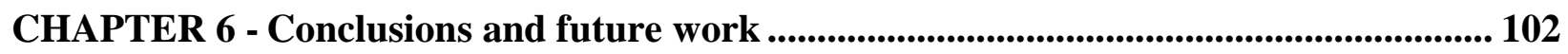

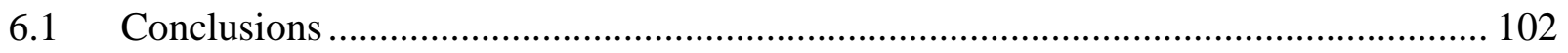

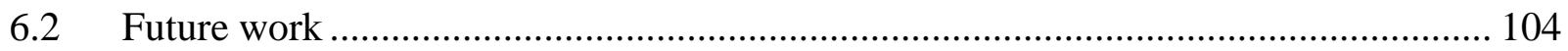

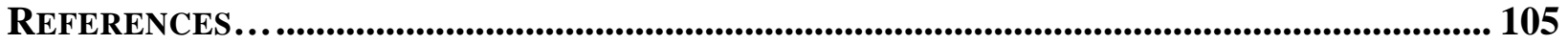

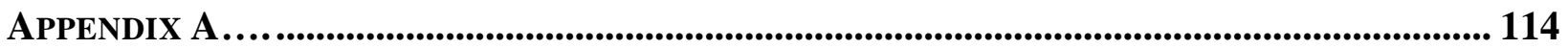

A.1 Comparison of planar cell and button cell............................................................. 114

A.2 Additional V-I curves for different part of the cell ................................................ 116 
A.3 Simulated time-traces of exciting current and resulting potential relaxation plots ......... 117

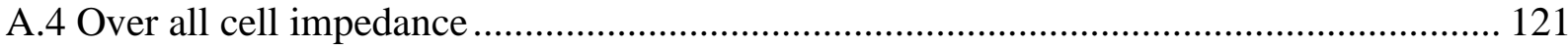

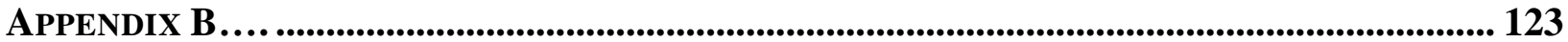

Phosphine induced Nickel Migration in SOFC Anodes: A Computational Study ................ 123

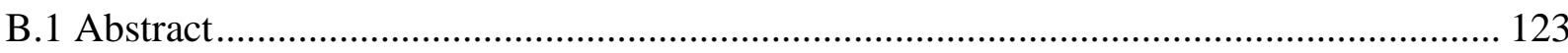

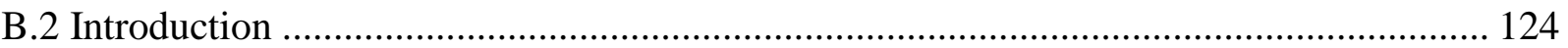

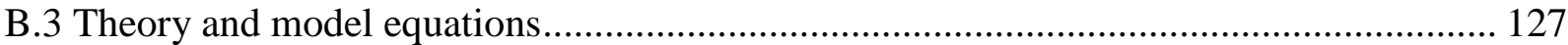

B.3.1 Integration with one dimensional degradation model ........................................ 127

B.3.2 Diffusion and formation of secondary phases ............................................... 130

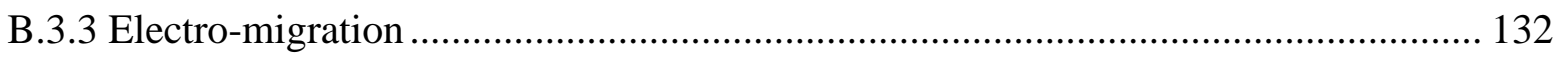

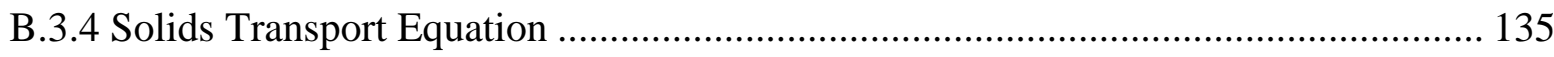

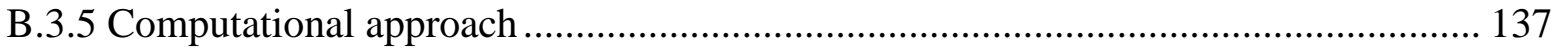

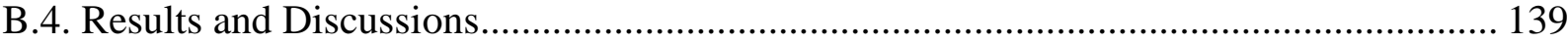

B.4.1 The influence of the Steam Concentration and Loading Current ........................... 144

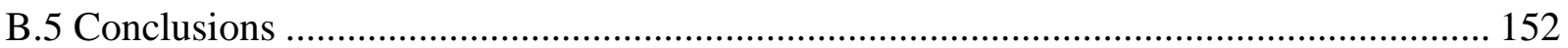




\section{LIST OF FIGURES}

Figure 1.1: World energy consumption by year, data adopted from Roper. [1] ....................... 2

Figure 1.2: World annual coal consumption, data adopted from Tverberg. [3]......................... 3

Figure 1.3: Species, electron and ion transport phenomena and reaction mechanism in SOFC.... 6

Figure 1.4: Oxygen vacancies inside SOFC dense electrolyte, YSZ [11] .............................. 7

Figure 1.5: Representation of typical triple phase boundary of a SOFC . ............................... 8

Figure 1.6: Contaminant transport and coverage formation for a SOFC exposed to fuel

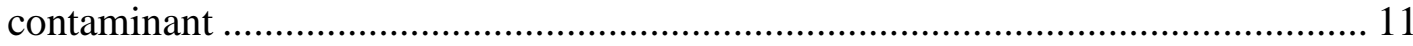

Figure 1.7: Fuel contaminant performance degradation model ........................................... 12

Figure 1.8: Computational domain for finite volume method (FVM). The terms, W, P, E, represent west, center and east nodes respectively. The terms, dwz, dez are the distance of west and east nodes from central node respectively and the term dpz is the length of control volume

Figure 3.1: (a) 1D Anode supported SOFC (b) Model dependency to contaminant's coverage. 25

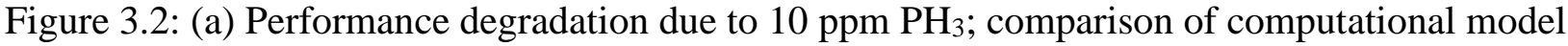
with experimental results for wet and dry fuel cases. (b) Losses during gradual degradation for wet fuel case predicted by the degradation model

Figure 3.3: (a) Normalized activation losses for wet and dry fuel cases (b) $\mathrm{PH}_{3}$ coverage at active layer for wet and dry cases

Figure 3.4: (a) Normalized $\mathrm{PH}_{3}$ coverage profile along the anode during gradual degradation time for wet fuel case. (b) Normalized porosity profile along the anode during the gradual degradation time for wet fuel case.

Figure 3.5: Predicted performance degradation for wet fuel case; performed for SOFCs with 250

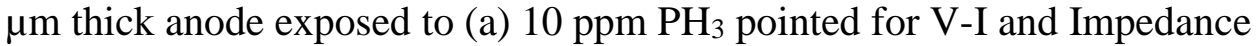
simulations (b) 10 ppm, 5 ppm and 2 ppm $\mathrm{PH}_{3}$

Figure 3.6: Predicted V-I (a) and impedance (b) curves at different times of phosphine exposure for wet hydrogen. 
Figure 4.1: a) The first derivative of the analytic function, $\phi$, with respect to u: using dual numbers (DNAD), finite differences (FD) and analytic derivative. b) the deviation of dual number (DNAD) and finite difference (FD) derivatives from analytic derivative.

Figure 4.2: a) The first derivative of analytic function, $\phi$, with respect to $\Gamma$ : using dual numbers (DNAD), finite differences (FD) and analytic derivatives. b) The deviation of dual (DNAD) and finite difference (FD) derivatives from analytic derivative.

Figure 4.3: a) The first derivation of analytic function, $\phi$, with respect to $C$ : using dual numbers (DNAD), finite differences (FD) and analytic function. $b$ ) The deviation of dual numbers (DNAD) and finite differences (FD) derivatives from analytic derivative 44

Figure 4.4: Error in derivative with respect to $u$ of the numerical solution on different grids using finite volume method with exponential scheme: a) Error in the Dual numbers (DNAD), b) Error in the finite differences (FD)

Figure 4.5: Flow chart of transient one-dimensional performance degradation model and its transport equations.

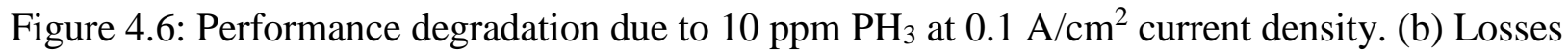
during gradual degradation for wet fuel case predicted by the degradation model. . 51

Figure 4.7: Dimensionless derivative of (a) electrical potential, (b) ohmic losses, (c) mass transfer losses (d) activation losses with respect to parameter $\mathrm{m}$ 52

Figure 4.8: Dimensionless derivative of (a) electrical potential, (b) ohmic losses, (c) mass transfer losses (d) activation losses with respect to parameter $n$

Figure 4.9: Dimensionless derivative of (a) electrical potential, (b) ohmic losses, (c) mass transfer losses $(\mathrm{d})$ activation losses with respect to parameter $\mathrm{p}$ 54

Figure 4.10: Dimensionless derivative of (a) electrical potential, (b) ohmic losses, (c) mass transfer losses $(\mathrm{d})$ activation losses with respect to parameter $\mathrm{q}$............................ 55

Figure 5.1: DREAM SOFC integration with degradation model 59

Figure 5.2: Geometrical representation of Channel and SOFC's anode by Stevenson et al. [63] 63

Figure 5.3: (EDS) area atomic concentrations profiles after $50 \mathrm{~h}$ of $\mathrm{AsH}_{3}$ exposure by Stevenson et al. [63]. 65

Figure 5.4: $\mathrm{AsH}_{3}$ concentration inside anode after $50 \mathrm{~h}$ of $1 \mathrm{ppm} \mathrm{AsH}_{3}$ 66

Figure 5.5: Normalized $\mathrm{AsH}_{3}$ coverage concentrations inside porous anode at 16, 33, and $50 \mathrm{~h}$ of anode exposed to $\mathrm{AsH}_{3}$. 
Figure 5.6: Normalized atomic concentrations profiles at $50 \mathrm{~h}$ of arsine exposure from EDS analyses by Stevenson et al. [63]. and normalized arsine coverage at $50 \mathrm{~h}$ of arsine exposure from simulations performed with two different arsine mass transfer coefficients (hmass $=1 \times 10-5$ and $1 \times 10-6)$ from fuel channel to porous anode

Figure 5.7: 3D-Schematic representation of the computational domain for three-dimensional planar SOFCs with 10 channels.

Figure 5.8: 3D-Schematic representation of the computational domain for three-dimensional planar SOFCs with one channel.

Figure 5.9: Transient variation of temperature (colored by red) and potential (colored by green)

Figure 5.10: Contours of current density at anode/electrolyte interface (top) and cathode/electrolyte interface (bottom) at steady state without phosphine exposure 75

Figure 5.11: Contours of current density $\left(\mathrm{A} / \mathrm{cm}^{2}\right)$ at inlet (top) and at outlet (bottom) in steady state without phosphine exposure 76

Figure 5.12: Contours of hydrogen and oxygen mass fraction at anode/electrolyte interface (top) and cathode/electrolyte interface (bottom) respectively at steady state without phosphine exposure

Figure 5.13: Cell voltage drop (top) and phosphine coverage formation at electrolyte/anode interface (bottom) during phosphine induced performance degradation.

Figure 5.14: Contours of hydrogen mass fraction at anode/electrolyte interface at steady state (top-left) after $2 \mathrm{~h}$ (top-right), $4 \mathrm{~h}$ (bottom-left) and $6 \mathrm{~h}$ (bottom-right) of phosphine exposure

Figure 5.15: Contours of oxygen mass fraction at cathode/electrolyte interface at steady state (top-left) after $2 \mathrm{~h}$ (top-right), $4 \mathrm{~h}$ (bottom-left) and $6 \mathrm{~h}$ (bottom-right) of phosphine exposure

Figure 5.16: Contours of phosphine coverage at $\mathrm{x} / 2$ (y-z plane) after $2 \mathrm{~h}$ (top), $4 \mathrm{~h}$ (middle) and 6 $\mathrm{h}$ (bottom).

Figure 5.17: Contours of current density $\left(\mathrm{A} / \mathrm{m}^{2}\right)$ at anode/electrolyte interface at steady state (topleft) after $2 \mathrm{~h}$ (top-right), $4 \mathrm{~h}$ (bottom-left) and $6 \mathrm{~h}$ (bottom-right) of phosphine exposure

Figure 5.18: Predicted V-I curves of planar cell at different times of phosphine exposure for dry hydrogen.

Figure 5.19: Planar cell partitioning for detailed impedance analysis. 89 
Figure 5.20: Time trace of exciting current and potential relaxation (exponential step $\tau=10-5$ ) for impedance analysis of planar cell simulated by DREAM-SOFC

Figure 5.21: Predicted impedance curves for planar cell in different regions (inlet, middle and outlet) at steady state condition of the cell: Nyquist representation (top) and Bode representation (bottom).

Figure 5.22: Predicted impedance curves for planar cell in different regions (inlet, middle and outlet) at $2 \mathrm{~h}$ of phosphine exposure: Nyquist representation (top) and Bode representation (bottom).

Figure 5.23: Predicted impedance curves for planar cell in different regions (inlet, middle and outlet) at $4 \mathrm{~h}$ of phosphine exposure: Nyquist representation (top) and bode representation (bottom).

Figure 5.24: Predicted impedance curves for planar cell in different regions (inlet, middle and outlet) at $6 \mathrm{~h}$ of phosphine exposure: Nyquist representation (top) and bode representation (bottom).

Figure 5.25: Hydrogen mole fraction (top) and Temperature (bottom) variation along the fuel channel at different times of phosphine exposure

Figure A.1: Predicted cell voltage under 10 ppm $\mathrm{PH}_{3}$ exposure: button cell (top), planar cell (bottom).

Figure A.2: Predicted V-I curves of planar cell at different times of phosphine exposure for dry hydrogen in different sections of the cell along the channel. 122

Figure A.3: Time trace of exciting current and potential relaxation (exponential step $\tau=10-4$ ) for impedance analysis of planar cell simulated by DREAM-SOFC in different regions (inlet, middle and outlet) at steady state condition of the cell.

Figure A.4: Time trace of exciting current and potential relaxation (exponential step $\tau=10-4$ ) for impedance analysis of planar cell simulated by DREAM-SOFC in different regions (inlet, middle and outlet) at $2 \mathrm{~h}$ of phosphine exposure

Figure A.5: Time trace of exciting current and potential relaxation (exponential step $\tau=10-4$ ) for impedance analysis of planar cell simulated by DREAM-SOFC in different regions (inlet, middle and outlet) at $4 \mathrm{~h}$ of phosphine exposure 125

Figure A.6: Time trace of exciting current and potential relaxation (exponential step $\tau=10-4$ ) for impedance analysis of planar cell simulated by DREAM-SOFC in different regions (inlet, middle and outlet) at $6 \mathrm{~h}$ of phosphine exposure 126

Figure A.7: Comparison of simulation and experiment at steady state condition ..... 127 
Figure A.8: Predicted impedance curves for planar cell at different time of phosphine exposure (initial, 2h, 4h, 6h): Nyquist representation (top) and bode representation (bottom)

Figure B.1: Integration of nickel migration and one dimensional performance degradation code.

Figure B.2: Nickel diffusion coefficients from lattice, grain boundaries and surfaces at high temperatures. 136

Figure B.3: Charged ions transport mechanism under the electron wind force due to the momentum transfer and electrical force due to the electrical field.

Figure B.4: (a) First stage of Ni-YSZ anode (b) second stage: formation of coverage (c) third stage: formation of secondary phases (d) fourth stage: nickel and secondary phases diffusion

Figure B.5: (a) One dimensional SOFC's anode geometry, (b) Discretization of computational domain....

Figure B.6: Initial conditions of anode (a) initial spatial species and porous volume distribution along anode. (b) Initial spatial Nickel mass (gram) distribution along anode. ....... 146

Figure B.7: Wet (\%25) fuel case; performed for SOFCs with $60 \mu \mathrm{m}$ thick anode exposed to 10 ppm $\mathrm{PH}_{3}$ under $0.25 \mathrm{~A} / \mathrm{cm}^{2}$ current loading (a) Predicted cell potential drop (b) Phosphine coverage distribution at different gradual degradation time.

Figure B.8: Wet (\%25) fuel case; performed for SOFCs with $60 \mu \mathrm{m}$ thick anode exposed to 10 ppm $\mathrm{PH}_{3}$ under $0.25 \mathrm{~A} / \mathrm{cm}^{2}$ current loading (a) anode porosity and (b) anode conductivity distribution at different gradual degradation time

Figure B.9: Wet (\%25) fuel case; performed for SOFCs with $60 \mu \mathrm{m}$ thick anode exposed to 10 ppm $\mathrm{PH}_{3}$ under $0.25 \mathrm{~A} / \mathrm{cm}^{2}$ current loading (a) surface diffusion inside anode (b) Spatial distribution of grain boundary diffusion at different gradual degradation time

Figure B.10: Wet (\%25) fuel case; performed for SOFCs with $60 \mu \mathrm{m}$ thick anode exposed to 10 ppm $\mathrm{PH}_{3}$ under $0.25 \mathrm{~A} / \mathrm{cm}^{2}$ current loading (a) variation of drift velocity inside anode (b) Spatial distribution of Nickel at different gradual degradation time .... 149

Figure B.11: Wet (\%25) fuel case; performed for SOFCs with $60 \mu \mathrm{m}$ thick anode exposed to 10 ppm $\mathrm{PH}_{3}$ under $0.25 \mathrm{~A} / \mathrm{cm}^{2}$ current loading (a) Ni-P phases (b) sum of Ni and Ni-P phases at different gradual degradation time along anode thickness. 150

Figure B.12: Comparison of the sum of $\mathrm{Ni}$ accumulation under wet $\left(25 \% \mathrm{H}_{2} \mathrm{O}\right)$ at $\mathrm{OCV}$ and 0.25 $\mathrm{cm}^{2}$ current load, and dry at $0.25 \mathrm{~cm}^{2}$ current load: (a) Region I (b) Region II .... 152 
Figure B.13: Comparison of the sum of Ni-P (secondary phases) accumulation under (25\% $\mathrm{H}_{2} \mathrm{O}$ )at $\mathrm{OCV}$ and $0.25 \mathrm{~cm}^{2}$ current load, and dry at $0.25 \mathrm{~cm}^{2}$ current load: (a) Region I, (b) Region II.

Figure B.14: Comparison of the sum $\mathrm{Ni}+\mathrm{Ni}-\mathrm{P}$ (secondary phases) accumulation under wet (25 $\% \mathrm{H}_{2} \mathrm{O}$ ) at $\mathrm{OCV}$ and $0.25 \mathrm{~cm}^{2}$ current load, and dry at $0.25 \mathrm{~cm}^{2}$ current load: (a) Region I, (b) Region II

Figure B.15: Comparison of the sum of $\mathrm{Ni}$ accumulation under wet $\left(3 \% \mathrm{H}_{2} \mathrm{O}\right)$ at $\mathrm{OCV}$ and 0.25 $\mathrm{cm}^{2}$ current load, and dry at $0.25 \mathrm{~cm}^{2}$ current load: (a) Region I, (b) Region II ... 155

Figure B.16: Comparison of the sum of Ni-P accumulation under wet $\left(3 \% \mathrm{H}_{2} \mathrm{O}\right)$ at $\mathrm{OCV}$ and $0.25 \mathrm{~cm}^{2}$ current load, and dry $0.25 \mathrm{~cm}^{2}$ current load: (a) Region I, (b) Region II 155

Figure B.17: Comparison of the sum $\mathrm{Ni}+\mathrm{Ni}-\mathrm{P}$ accumulation under wet $\left(3 \% \mathrm{H}_{2} \mathrm{O}\right)$ at $\mathrm{OCV}$ and $0.25 \mathrm{~cm}^{2}$ current load, and dry $0.25 \mathrm{~cm}^{2}$ current load: (a) Region I, (b) Region II 156

Figure B.18: The sum of Ni-P (secondary phases) accumulation under $\left(25 \% \mathrm{H}_{2} \mathrm{O}\right)$ at $\mathrm{OCV}$ and $0.25 \mathrm{~cm}^{2}$ current load, and dry at $0.25 \mathrm{~cm}^{2}$ current load in region I. 157

Figure B.19: The sum $\mathrm{Ni}+\mathrm{Ni}-\mathrm{P}$ (secondary phases) accumulation under wet $\left(25 \% \mathrm{H}_{2} \mathrm{O}\right)$ at OCV and $0.25 \mathrm{~cm}^{2}$ current load, and dry at $0.25 \mathrm{~cm}^{2}$ current load in Region I ... 158 


\section{LIST OF TABLES}

Table 3.1: Model parameters: $\mathrm{kf}, \mathrm{x}, \mathrm{kb}, \mathrm{x}$ are forward and backward reaction rate of species $\mathrm{x}$ respectively, $\mathrm{m}, \mathrm{n}, \mathrm{p}, \mathrm{q}$ are model parameters.

Table 4.1: Model parameters and cell operating conditions 46

Table 5.1: Model parameters used for $\mathrm{AsH}_{3}$ degradation and test conditions for the PNNL experiments

Table 5.2: Geometry and operating conditions parameters for planar SOFC

Table 5.4: Roughly impedance calculations at low and high frequencies

Table 5.5: Polarization and ohmic resistance of the cell at operating current load in different times of degradation

Table B.1: Conditions and parameters for simulations 


\begin{tabular}{|c|c|}
\hline As & arsenic \\
\hline$a_{E}$ & east computational node \\
\hline$a_{P}$ & center computational node \\
\hline$a_{W}$ & west computational node \\
\hline$D_{\theta}$ & transport coefficient, $\mathrm{m}^{2} \cdot \mathrm{s}^{-1}$ \\
\hline$D_{\text {eff }}$ & effective diffusion coefficient, $\mathrm{m}^{2} \cdot \mathrm{s}^{-1}$ \\
\hline$E_{a c t, i}$ & activation energy barrier, J.mole ${ }^{-1}$ \\
\hline$E_{N}$ & Nernst potential, $\mathrm{V}$ \\
\hline$F$ & Faraday’s constant, C.mole ${ }^{-1}$ \\
\hline$i$ & total current density, A.m ${ }^{-2}$ \\
\hline$i_{\text {ref }}$ & reference current density, A.m ${ }^{-2}$ \\
\hline$i_{o}$ & exchange current density, A.m ${ }^{-2}$ \\
\hline$i_{F}$ & Faradic current \\
\hline$k_{f, H}, k_{b, H}$ & $\begin{array}{l}\text { forward and backward reaction rate constants } \\
\text { for hydrogen adsorption, } \mathrm{s}^{-1}\end{array}$ \\
\hline$k_{f, X}, k_{b, X}$ & $\begin{array}{l}\text { forward and backward reaction rate constants } \\
\text { for contaminant } X \text { adsorption, } \mathrm{s}^{-1}\end{array}$ \\
\hline
\end{tabular}




\begin{tabular}{|c|c|}
\hline$h_{m}$ & mass transfer coefficient, $\mathrm{m} . \mathrm{s}^{-1}$ \\
\hline $\mathrm{m}$ & degradation model parameter \\
\hline $\mathrm{n}$ & degradation model parameter \\
\hline $\mathrm{Ni}$ & nickel \\
\hline Ni-P & nickel-phosphate phases \\
\hline$P$ & pressure of the system, $\mathrm{Pa}$ \\
\hline $\mathrm{P}$ & phosphorous \\
\hline $\mathrm{p}$ & degradation model parameter \\
\hline q & degradation model parameter \\
\hline$R_{u}$ & universal gas constant, J.mol ${ }^{-1} \cdot \mathrm{K}^{-1}$ \\
\hline$R_{i}$ & $\begin{array}{l}\text { molar production rate of species } i \text { due to } \\
\text { homogenous reactions, mole. } \mathrm{m}^{-3} \cdot \mathrm{s}^{-1}\end{array}$ \\
\hline$u$ & drift velocity $\mathrm{m} / \mathrm{s}$, convection velocity \\
\hline$S_{i}$ & $\begin{array}{l}\text { net production rate of species } i \text { due to surface } \\
\text { reactions, mole. } \mathrm{m}^{-3} \cdot \mathrm{s}^{-1}\end{array}$ \\
\hline$T$ & temperature of the system, $\mathrm{K}$ \\
\hline$T_{m}$ & melting temperature, $\mathrm{K}$ \\
\hline $\mathrm{t}$ & time, $\mathrm{s}$ \\
\hline
\end{tabular}

\section{Greek}

$\begin{array}{ll}\alpha & \text { transfer coefficient } \\ \delta & \text { anode thickness, } \mathrm{m}\end{array}$


porosity

$\varepsilon_{0}$

porosity at zero percent contaminant coverage

$\phi \quad$ electrical potential, $\mathrm{V}$

$\eta_{i} \quad$ overpotentials, $\mathrm{V}$

$\Gamma \quad$ transport coefficient , mol.m $\mathrm{m}^{-2}$

$\theta_{i} \quad$ surface coverages

$\sigma \quad$ electrical conductivity, S.m ${ }^{-1}$

$\sigma_{0}$

electrical conductivity at zero percent contaminant coverage, S.m ${ }^{-1}$

$\omega_{\theta_{i}} \quad$ source terms due to surface adsorption reactions 


\section{CHAPTER 1 - Introduction}

\subsection{Motivation}

Energy is available in nature in different forms. Matter itself is the physical vessel containing energy; in many important cases is stably held in natural reservoirs including coal, oil, and natural gas. Therefore, it is necessary to transform energy from a less useful state (bound chemical energy) to a more useful state (electricity). Initially, this transformation was carried out by burning the matter. In the last few centuries, within the industrial revolution, the energy was first obtained by combustion of natural fossil fuels products, like coal and petroleum.

If one just takes a moment and thinks how much energy is needed to survive in this modern time, then easily he/she can conclude that energy is needed for every movement that we have in our daily life. In all manufacturing, transportation, telecommunication, lighting industries and health care systems, energy is required. Our modern life and the developed industry have increased the energy demand rapidly in last century and it is estimated that it will increase faster in the next century (Figure 1.1). It is important to decide which technique is convenient to transform energy from matter to its useful forms. As it is mentioned earlier, in the last century, combustion of fossil fuels has been used to obtain useful form of energy. Especially, a rapid increase in coal consumption is observed over last two centuries (Figure 1.2). 


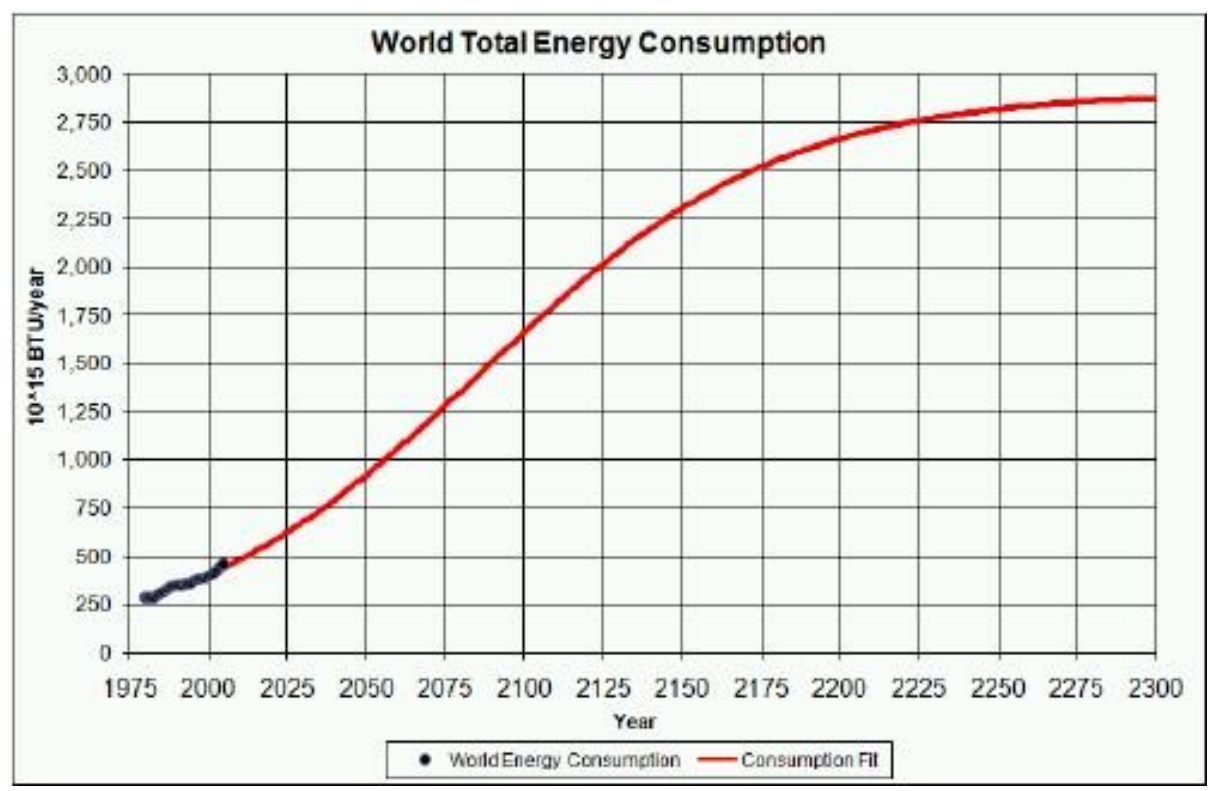

Figure 1.1: World energy consumption by year, data adopted from Roper. [1]

Combustion of fossil fuels releases pollutants which may cause risks for human health and the environment. Our modern life requires an increase in energy consumption, and our health requires a clean environment that is not polluted. So, in order to compensate the requirements of both a healthy and modern life, an alternative energy generation method should be investigated. At this point, fuel cells, and in particular solid oxide fuel cells (SOFCs), are one alternative that can be considered to meet these requirements. SOFC or in more general form, a fuel cell, is a device that converts chemical energy of a fuel,(e.g. hydrogen and carbon monoxide) into electricity via an electrochemical reaction. SOFCs can be operated directly on a variety of fuels, including coal syngas. Since coal is the most abundant [2] and economically available fuel in the world, it is desirable to use coal-syngas in SOFC to generate clean power from coal. 


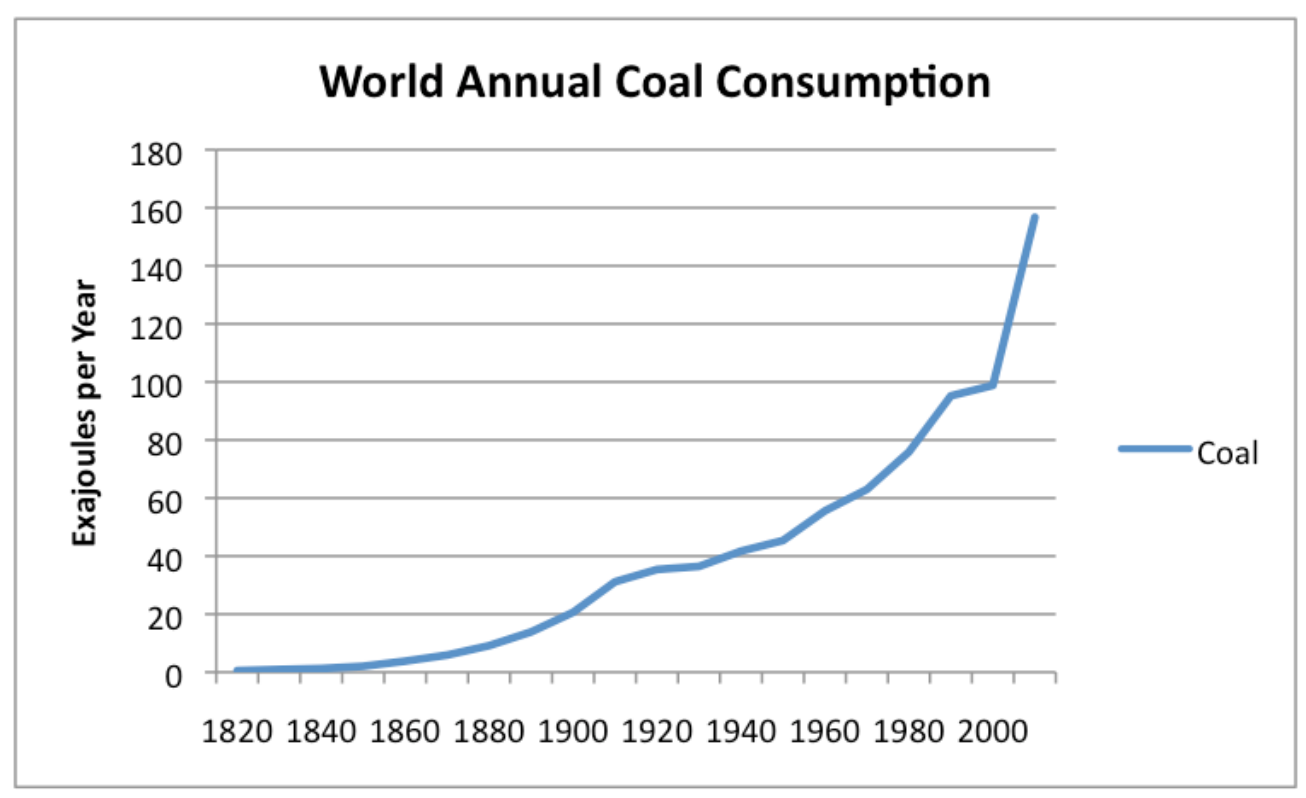

Figure 1.2: World annual coal consumption, data adopted from Tverberg. [3]

However, coal syngas operated SOFCs have significant performance losses due to the coal syngas impurities such as phosphine, arsenic, zinc and selenium. Contaminants in low levels (1 ppm-20 ppm) inside the fuel react chemically with the catalyst materials which causes deactivation of the catalyst and also can lead the migration of the catalyst from the electrochemically active region to anode open surface (fuel channel/anode interface). In SOFCs the electrochemical reaction occurs on a layer (electrochemically active layer) that is close to the anode/electrolyte interface. The catalytic activity of this layer is crucial for sustainable performance of the SOFCs.

There are two basic SOFCs design, the electrolyte-supported and electrode supported. The first one is electrolyte supported SOFC consists of a thick electrolyte that has both sides coated with a thin electrode. The anode supported cell which has a thick anode that has one side coated with thin electrolyte and cathode. Cathode supported SOFC's are rare but a few examples can be also found in the literature. Both anode supported and electrolyte supported SOFCs will be used in this study. It is important to mention that the anode supported cell has advantage due to its smaller ohmic 
resistance as compared to the electrolyte supported design. The ohmic losses are mainly because of the ionic conductivity of electrolyte. They can be reduced by decreasing the thickness of the electrolyte. Investigation of the contaminant performance degradation of SOFCs is vital in the pursuit of sustainable energy power generation systems. Many experimental and computational works have been performed to predict the fuel contaminant performance degradation of the SOFCs $[4,5,6,7]$. Experimental works have also been carried out to analyze the behavior and the chemical characteristic of the contaminant degradation of anode supported cell with phosphine, arsenic, selenium and the other poisoning elements $[8,9,10]$. Experimental results and phase diagrams show that contaminants form different chemical compounds with the nickel catalyst. Consequently, their effects on the performance of the cell need to be investigated in detail.

Mathematical models can reveal important features of a system that can be used during the research and development stage of any product. Mathematical models are usually cheaper and often faster than experiments. Experiments are always required to validate a mathematical model, but once the computational model is validated it can be used to study different cases without need of a new experiment.

The motivation behind this work is the need of such computational model that can help the designers to design and develop efficient and durable SOFCs that are operated with coal syngas.

\subsection{The Solid Oxide Fuel Cell (SOFC)}

The Solid oxide fuel cell (SOFC) is an electrochemical device that converts chemical energy of a fuel into electricity. It has three main components which are anode, electrolyte and cathode. The porous anode of a SOFC is a mixed ionic and electronic conductor which is also used as a catalyst 
to accelerate the rate of electrochemical reaction by decreasing the activation energy of the reaction that is occurring between anode/electrolyte interfaces. A commonly used anode material is made of nickel/yittria-stabilized zirconia (Ni/YSZ) cermet. Nickel is used as a catalyst and electron conductor, whereas YSZ is used to increase the oxygen ionic conductivity inside the active layer so that the triple phase boundary (TPB) will increase. TPB is very important for the reaction to occur and it is defined as the three phase boundary of gas phase, electrolyte and electronic conductor. The dense electrolyte of a SOFC is a good oxygen ion $\left(\mathrm{O}^{2-}\right)$ conductor and electron insulating ceramic membrane that is usually made of YSZ. Cathode material of a SOFC is also a mixed ionic and electronic conductor. The inter-connect of a SOFC should be electronically conductive and it should avoid any gas leakage from the SOFC's stack. Since the SOFC is a high temperature fuel cell, its components should be designed so that a sustainable performance can be obtained.

Species transport, ionic and electrical conductivity, and chemical reaction mechanism in SOFC (Figure 1.3) are the most important physical phenomena that are essentially responsible for the performance and durability of the cell. These areas have been investigated by many research groups over the last several decades. 


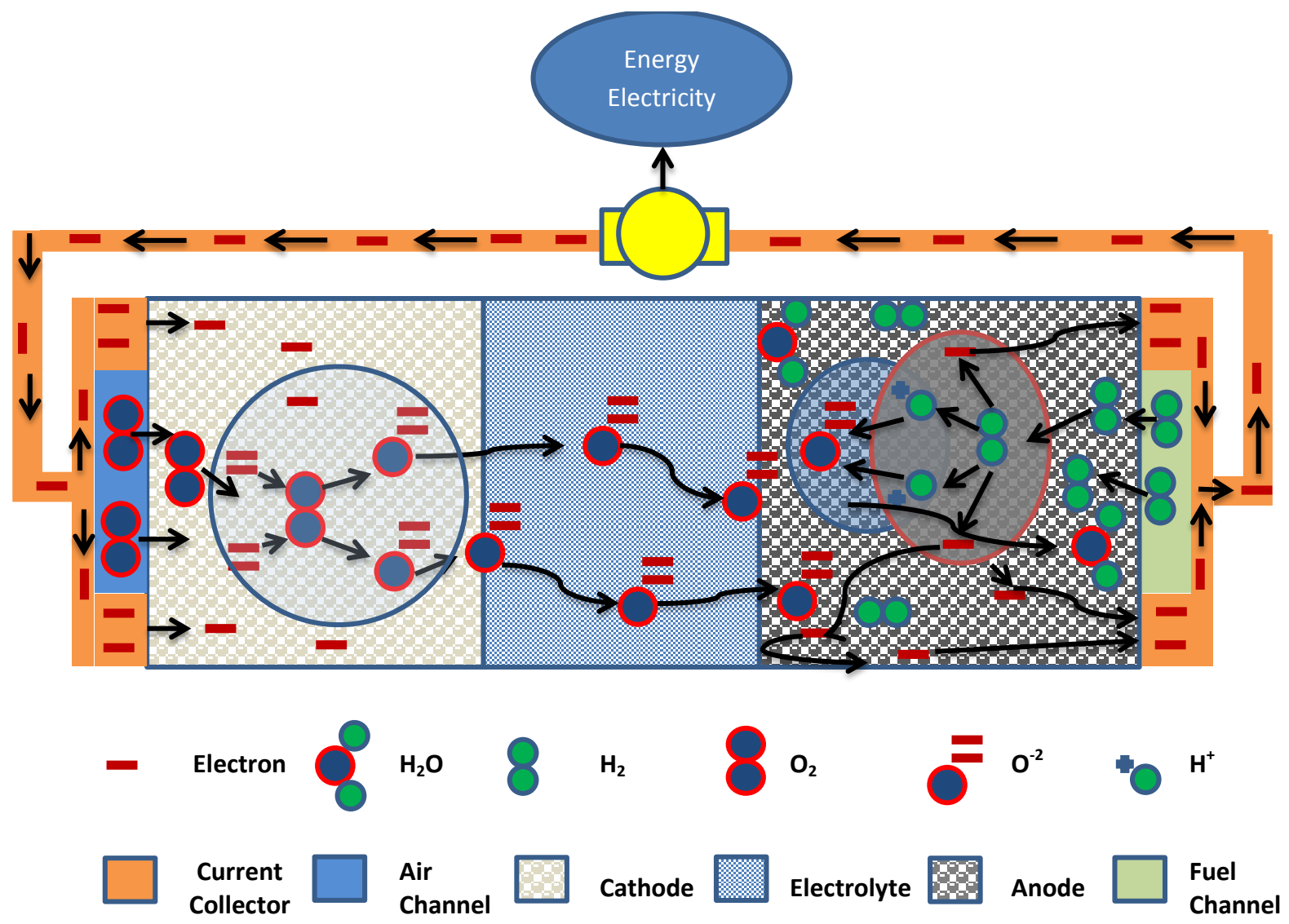

Figure 1.3: Species, electron and ion transport phenomena and reaction mechanism in SOFC

The transport phenomena in SOFC are composed of species transport of oxidizer and fuel. Oxidizer and fuel transport occur inside the cathode and anode, respectively. Both cathode and anode materials must be porous to allow species transport and must have good mechanical properties (optimum porosity) so that the cell can work properly in high temperature conditions. Concentration losses are attributable to the transport of species. When the cell operating current density is increased, there will be a consumption of the oxidizer and fuel. This consumption causes partial pressure drop, which in turn leads a drop in overall cell voltage. This drop in cell voltage is the so called concentration over-potential. 
Ionic and electrical conductivity are the physical properties that are responsible for the ohmic losses. Ions are transported through the dense electrolyte. Electrolyte material design is very important for fuel cells. In SOFC, a solid dense ceramic is used as an electrolyte and ionic transport occurs via electrolyte's available oxygen vacancies as it is shown in Figure 1.4. A thinner electrolyte can ease the transport of ions; therefore, the ohmic loss due to ionic transport can be reduced. Electrons are created inside the anode via half-cell reaction Eq. (1), and then the created electrons travel in an electronically conductive path which is composed of anode, current collector and cathode (Figure 1.3). Electrons are captured by $\mathrm{O}_{2}$ to produce oxygen ions $\left(\mathrm{O}^{-2}\right)$ via half-cell reaction in cathode. The conductive path must provide high electronic conductivity so that ohmic loss due to electronic conduction can be reduced.

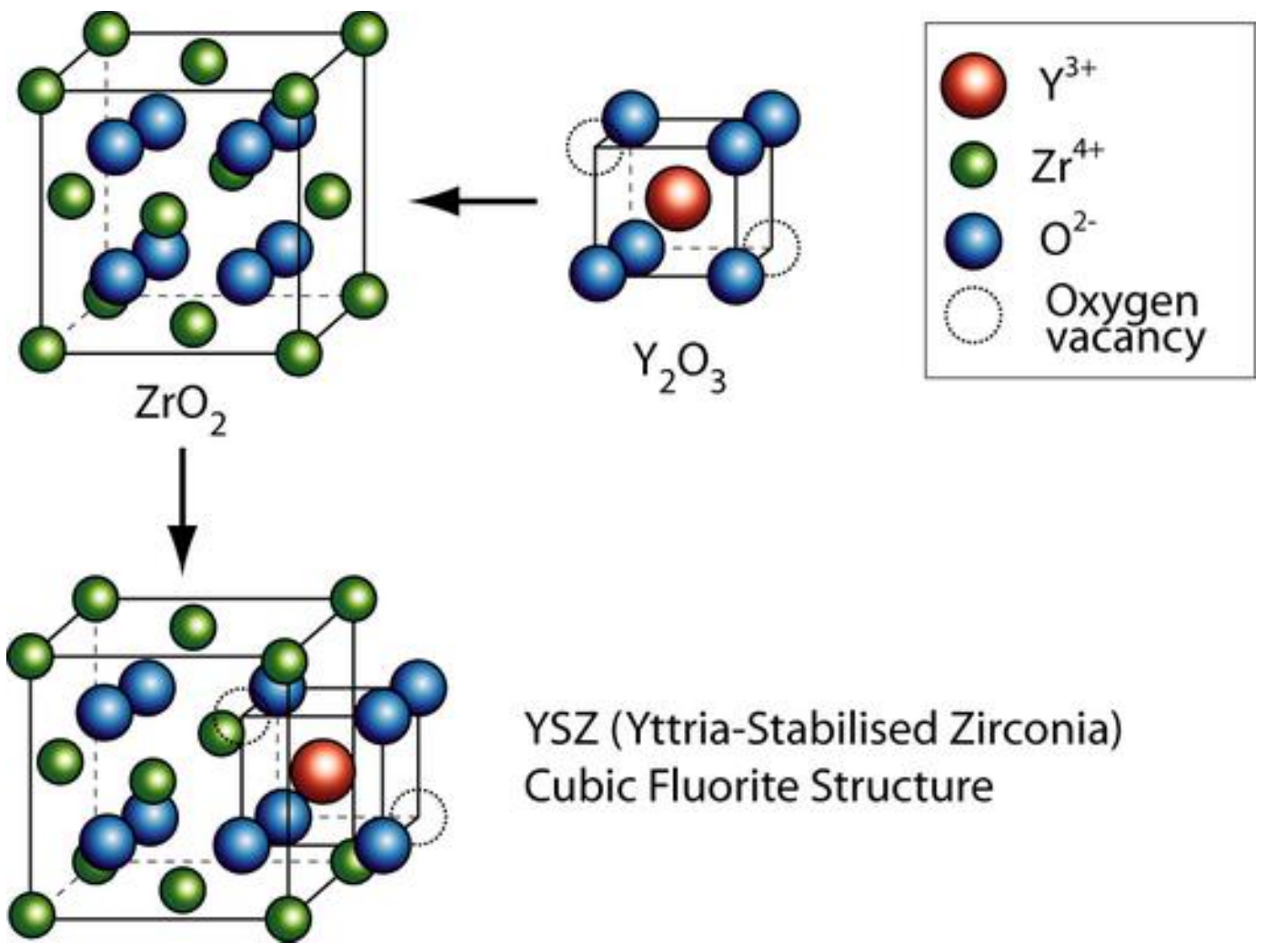

Figure 1.4: Oxygen vacancies inside SOFC dense electrolyte, YSZ [11] 
A chemical reaction mechanism is the process in which chemical energy is converted to electricity directly and exhaust species are produced. These reactions are half-cell electrochemical reactions Eqs. (1.1) and (1.2) which take place in anode and cathode, respectively, and overall cell chemical reaction (Eq. (1.3)) that occurs in the cell.

$$
\begin{aligned}
& \mathrm{H}_{2}+\mathrm{O}^{=} \underset{\leftarrow}{\leftarrow} \mathrm{H}_{2} \mathrm{O}+2 e^{-} \\
& \frac{1}{2} \mathrm{O}_{2}+2 e^{-} \stackrel{\rightarrow}{\leftarrow} \mathrm{O}^{=} \\
& \mathrm{H}_{2}+\frac{1}{2} \mathrm{O}_{2} \stackrel{\rightarrow}{\leftarrow} \mathrm{H}_{2} \mathrm{O}
\end{aligned}
$$

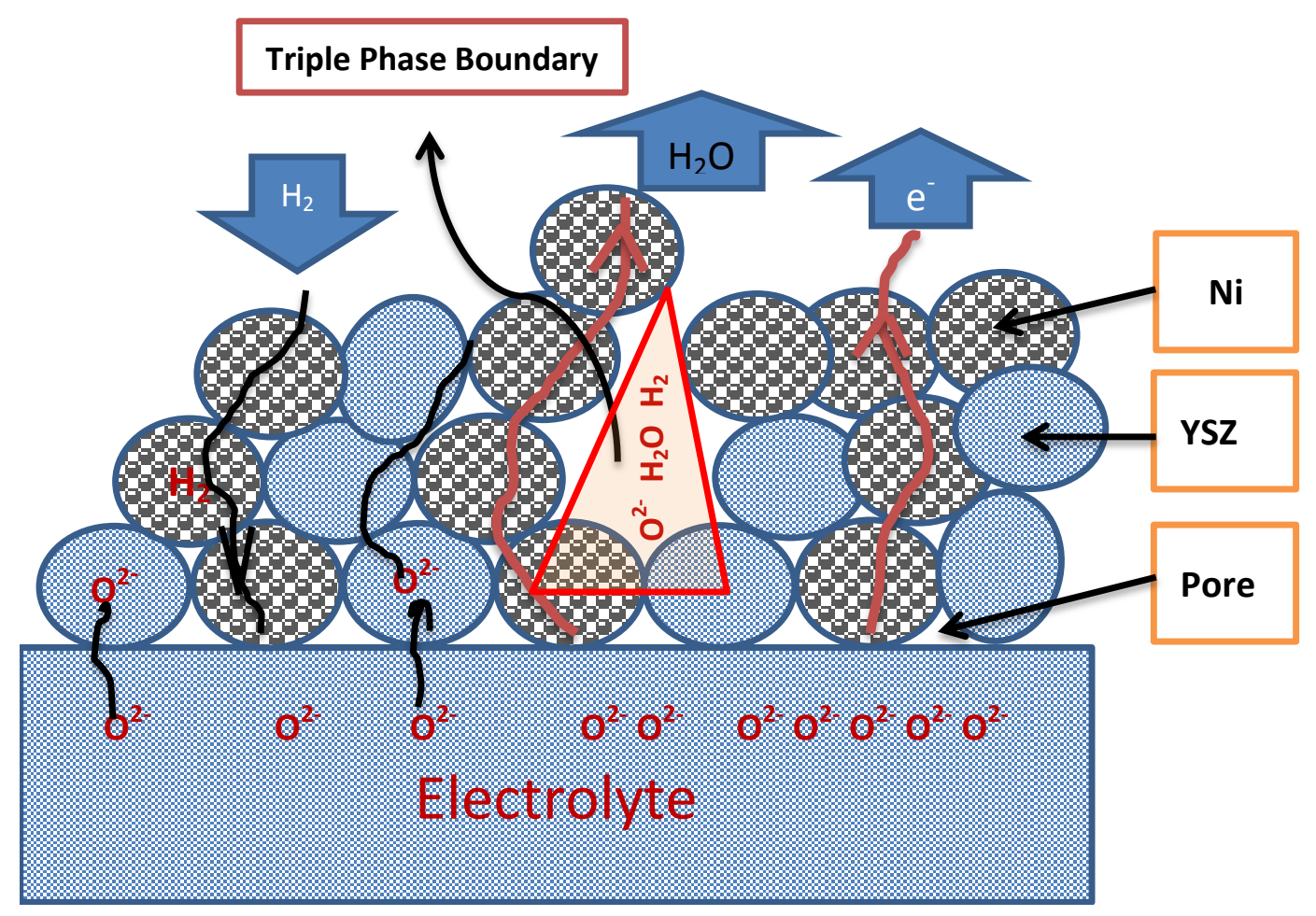

Figure 1.5: Representation of typical triple phase boundary of a SOFC. 
This phenomenon essentially governs the activation over potential which is related to speed of electrochemical reaction. The rate of electrochemical reactions is very important for fuel cells to sustain high performance. There are several ways to enhance the speed of the electrochemical reactions, such as using a catalyst, increasing operation temperature, enhancing the surface of catalyst porosity, raising the partial pressure of reactants and others. For SOFC, if the catalyst material and the operation temperature are known, then to improve the speed of electrochemical reaction, the TPB and partial pressure of reactants must be enhanced. Partial pressure of reactants can be increased by enhancing the transport of reactants by increasing porosity. TPB can be increased by increasing porosity, contact area between catalyst and ionic conductor as it is shown in Figure 1.5.

\subsection{Fuel contaminant degradation of SOFC}

The utilization of coal syngas in SOFC makes the fuel contaminant performance degradation of SOFC an important area. This is the main objective of the current study. When SOFC is utilized with coal syngas with impurities even at low concentrations $(\leq 10 p p m)$, its performance decreases dramatically due to the various physical and chemical phenomenon that take place inside the anode. These phenomena have been investigated over the last decade by experimental observations. However, long term experiments are required at low contaminant concentration for proper life cycle analysis. Therefore, as it is the case for most scientific problems, development of computational models could reduce the cost and time of fuel contaminant performance degradation experiments. It is essential to emphasize that computational models cannot replace experiments or vice-versa. Actually, one could be used to complement the other. 
In order to predict experimentally observed degradation behavior of SOFC's anode due to fuel impurities, a one-dimensional phenomenological computational model was developed by Cayan et al. [12]. This model is developed based on the following itemized list. Transport phenomena and chemical reaction mechanism of the model are depicted in Figure 1.6.

i. Impurity molecules are chemically active to form secondary phases along the anode thickness.

ii. These secondary phases (contaminant coverage) are assumed to block the active sites of catalytic material. This causes a decrease in the kinetic rate of half-cell reaction that takes place inside anode. Therefore the activation overvoltage increases rapidly.

iii. Contaminant coverage and secondary phase formation are believed to block pores inside the anode, hence effective diffusion of gaseous species and triple phase boundary decreases. This causes an increase in concentration over potential and activation over voltage. 

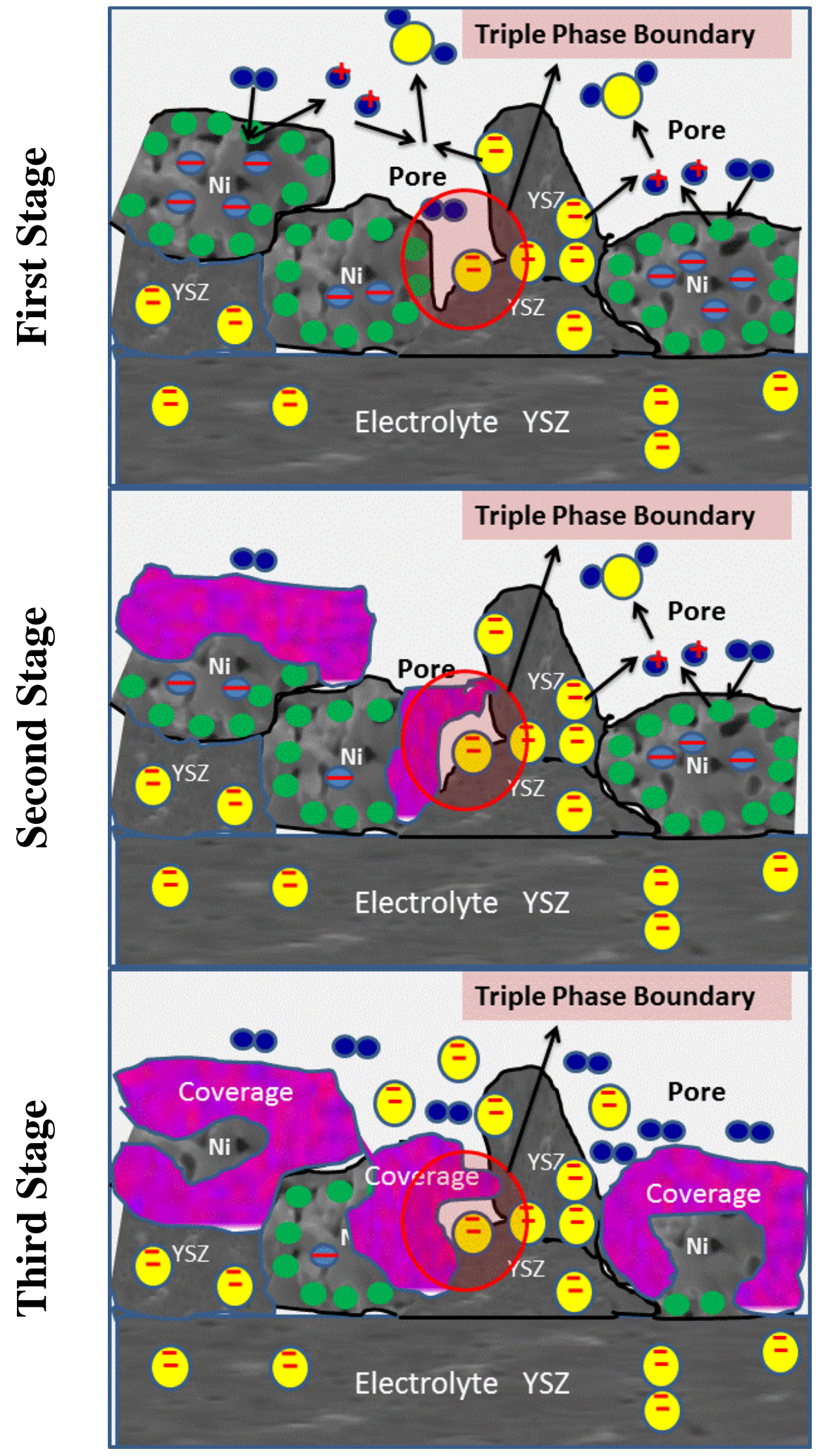

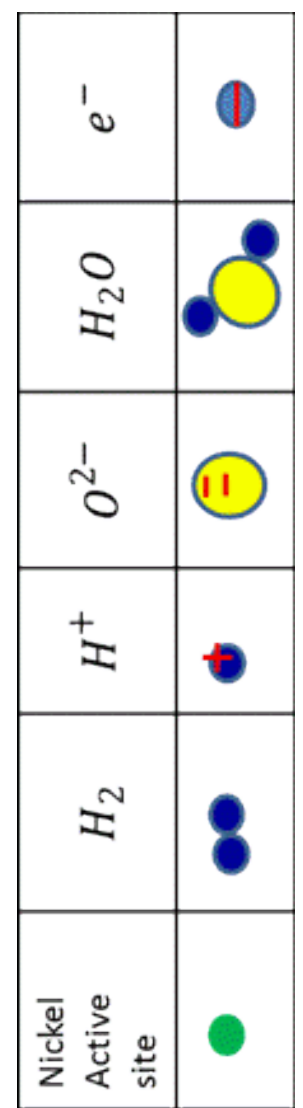

Figure 1.6: Contaminant transport and coverage formation for a SOFC exposed to fuel contaminant 
iv. Coverage changes the structure of the anode. This is believed to decrease the electronic conductivity of the cell, hence an increase in ohmic losses. This effect is neglected in this study, because work is focused on phosphine-induced degradation, and the secondary phases formed have high enough electronic conductivity to sustain electron conduction with a negligible increase in ohmic resistance.

Model equations, model parameters and their dependency are depicted in Figure 1.7. Degradation model is developed based on the following order. Initialization is performed on species concentration, coverages (contaminants and fuel), cell current density, and cell potential.

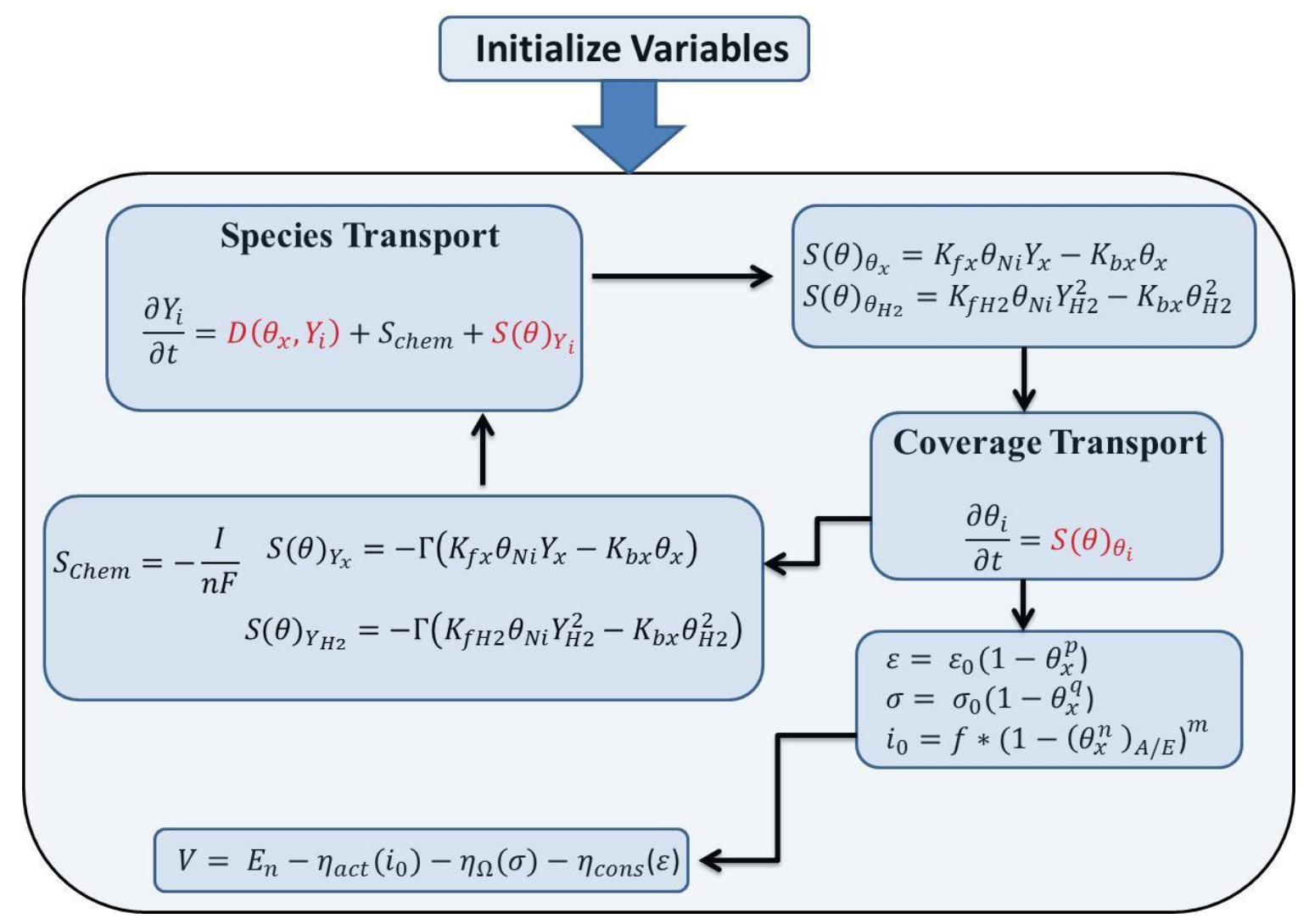

Figure 1.7: Fuel contaminant performance degradation model 
Next, species transport equations are solved. Then, the source terms for the coverage equation are calculated using species concentrations. Once coverage is solved, three model properties, porosity, conductivity and exchange current density are recalculated using new contaminant coverage by calibrated model parameters, $p, q, m$ and $n$. It should be noted here that, exchange current density is only a function of local impurity coverage at the active layer (anode/electrolyte interface). Further, the cell potential is calculated via the Poisson equation and polarization losses are calculated using these three model properties.

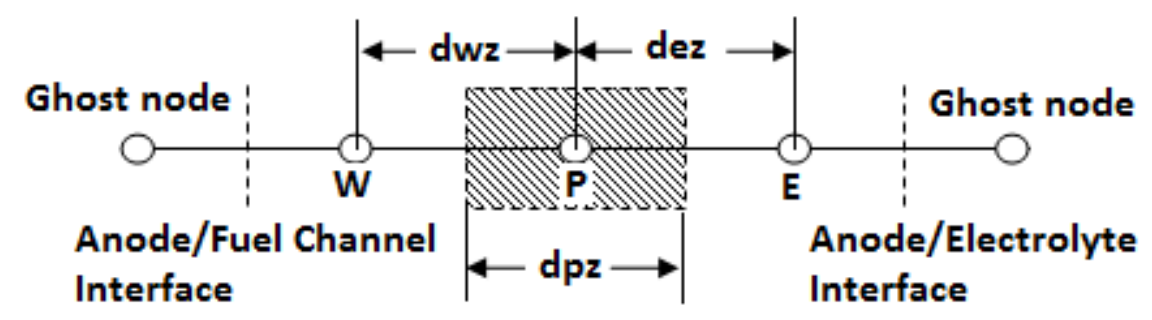

Figure 1.8: Computational domain for finite volume method (FVM). The terms, W, P, E, represent west, center and east nodes respectively. The terms, dwz, dez are the distance of west and east nodes from central node respectively and the term dpz is the length of control volume.

Species and coverage transport and electrical potential equations are solved using finite volume method (FVM). This method is a discretization technique that solves partial differential equations by integration over finite control volumes as depicted on Figure 1.8. The method is convenient for governing equations that are derived from conservation properties like mass, momentum and energy and it is highly recommended in fluid flow and heat transfer computational models. The right hand side of species transport equation is composed of three parts: diffusion, chemical and coverage source terms. The diffusion term is a function of contaminant's coverage via effective diffusion coefficient. The effective diffusion coefficient is a function of the porosity. The porosity 
decreases as contaminant coverage increases, hence diffusion losses increase. The electrochemical source term, proportional to current, is due to the consumption of the fuel at the electrochemically active area; therefore it is a negative source term for the fuel. The coverage source term is calculated from the adsorption-desorption surface reaction mechanism. It represents the amount of fuel and contaminant in the solid phase. Therefore, it is subtracted from fuel species concentrations in the transport equation. Both electrochemical and coverage source terms make the species transport equation stiff. Stiffness occurs often in scalar diffusion reaction equations. The Poisson equations for potential and coverage transport equations are not stiff; however, since contaminant coverage alters the porosity, conductivity and exchange current density, it causes stiffness also in these equations. 


\section{CHAPTER 2 - Literature review, background and objectives}

This chapter is divided in to four sections along associated with primary contributions of this study.

\subsection{One dimensional degradation model and polarization, impedance analysis}

Globally the amount of the coal reservoirs are much higher than the other fossil fuels such as petroleum and natural gas, therefore it has been estimated that there is going to be an increase of coal consumption into the next decades $[6,13]$. However, an alternative way to the conventional combustion process of the coal needs to be developed, to provide relatively cleaner energy from the coal. This is the reason why utilization of gasified coal in SOFCs has become a major research area. SOFCs are electrochemical devices that generate electricity directly from a wide variety of fuels such as biogas, hydrocarbons and coal syngas with high efficiency and reduced environmentally harmful emissions. They operate at relatively high temperatures $\left(\sim 800{ }^{\circ} \mathrm{C}\right)$ leading to the exhaust gas stream conditions that are ideal for use in combined heat and power applications and combined-cycle electric power plants [6,14]. However studies $[6,12,15,16,17,18,19,20,21]$ show that coal syngas operated SOFCs exhibit significant performance losses due to the impurities (phosphine, arsenic, zinc and selenium) in coal syngas. The impurities typically interact with the SOFC anode to form chemical species with nickel $[6,16,22]$ causing degradation of cell performance and eventual failure. The effects of the 
impurities vary with the type of the impurity molecule and other operating conditions of the fuel cell $[17,19,20,23,24]$. Recent studies on the effect of steam on phosphine induced degradation show that the degradation rate is lower when no steam is added to the fuel compared to when the fuel is humidified $[19,20]$

In this study, the experimentally observed influence of $\mathrm{PH}_{3}$ on the performance degradation under different steam concentrations is simulated using a phenomenological model. The mathematical model used in this study is an in-house code which solves for the distributions of gas phase specie concentrations, specie coverages on active sites and the electrical potential along the anode thickness. It is postulated that the impurity coverage on $\mathrm{Ni}$ sites marks the inception of performance degradation since it leads to subsequent formation of secondary phases with Ni thus altering the porosity, conductivity and the electrochemical activity of the anode. The effect on the porosity is modeled based on the experimental observations which show that the secondary phases block the pores. Reduced porosity could decrease the effective diffusivity of the fuel, thus the mass transport losses could increase due to the slow transport of the fuel to the active layer where electro chemical reactions occur. Although the electrical conductivity is also modeled as a function of the coverage, it is observed that the electrical conductivity does not have significant effect on the performance degradation. In order to simulate the experimentally observed effect of steam concentration, the available model is modified using the experiments conducted at National Institute of Fuel Cell Technology (NIFT), West Virginia University [19] as a guide. The experiments presented in $[15,20]$ show that the steam in the fuel accelerates the $\mathrm{PH}_{3}$ induced degradation process and causes changes in microstructure of the anode. Hence, the model developed in the present study is modified to account the presence of steam in the fuel. It was experimentally observed $[15,20]$ that the variation of porosity due to the coverage is higher when steam is added to the fuel. The second 
modification is the exchange current relation which is revised to make exchange current density a function of steam concentration as well based on studies $[25,26,27]$ which show that the exchange current density of hydrogen is a function of the steam concentration.

In order to assess the overall performance of the cell at different stages during the gradual degradation of the anode supported cells, a new simulation scheme is setup where the model predicts the V-I behavior and impedance response of the cell at any given time using the condition of the anode at that instant. These calculations are based on a cell level model which includes anode, electrolyte and cathode components of the button cell. The charge transfer inside composite electrodes is assumed to be occurring through the thickness of the electrode. The model treats each individual phase inside the composite electrolyte (electron conducting phase, ion conducting phase and pore phase) as separate continuums which are all mixed together and interact with each other at the local level through electrochemical reactions. The model treatment and assumptions are similar to those used in similar studies from literature [27,28].

The original model allows for charge transfer to occur away from the active interface, however this aspect is disabled for the simulations presented in this study and the charge transfer reaction is limited to the electrode/bulk electrolyte interfaces. This was done in order to be consistent with the electrochemistry model used in the code that predicts the cell degradation. After this modification, the parameters in the model are calibrated to obtain reasonable agreement with measured polarization and impedance curves of a real button cell [29]. The corresponding parameters in the degradation model are matched with the calibrated parameters and it is verified that the simulated anode polarization from the degradation model at zero time matched that from the button cell code. After this the predicted distributions of anode porosity and anode conductivity at different times obtained from the degradation code are input to the button cell code and the 
polarization and impedance curves at that stage are predicted following the procedure described above.

\subsection{Sensitivity analysis of the model parameters}

Sensitivity analysis can be used in design optimization using computational modeling, parameter estimation through calibration with experiments and assessment of uncertainty in the predictions. A commonly used measure of uncertainty is the partial derivative of the model output with respect to a model parameter which gives a 'local' sensitivity of the model near the nominal value of the parameter at which the derivative is evaluated.

Several methods are available for differentiating (i.e. evaluating derivatives of a dependent variable w.r.t model parameter used in the code) a computational model with respect to a given parameter, among which dual number automatic differentiation (DNAD) has attracted much interest lately [30-38]. DNAD utilizes the inherent compatibility of the chain rule of differentiation with the way computations are performed in a computer code, wherein all mathematical functions are successively broken down into a few basic math operations [39]. The implementation of DNAD requires attaching an additional component for storing the derivative (thus the terminology dual number) to all dependent variables and replacing all basic math operations involving the dual numbers with new ones, which calculate the derivative component. It is also possible to calculate derivatives with respect to more than one independent variable simultaneously by adding more components to the dependent variables. Several implementations of DNAD in different programming languages are available in literature which can be used to convert any existing code to a DNAD enabled version. In FORTRAN, for example, two popular open source libraries for adding DNAD capabilities to an existing code are ADIFOR [40] and AUTO_DERIV [41], which 
conform to FORTRAN 77 and FORTRAN 90 standards respectively. ADIFOR, especially, has been available for a long time and it has been successfully integrated with specialized research codes $[30,42]$ as well as with commercial $[33,34]$ CFD software.

When DNAD is applied correctly to a computer code the result usually is a code which simultaneously evaluates the dependent quantities and their derivatives with some additional cost of some additional computations and memory space [39]. The computed values are the precise derivatives, to the machine accuracy, of the mathematical functions evaluated to calculate the dependent quantities. In other words, the sensitivities from DNAD are those of the numerical solution to the mathematical model rather than those of the mathematical model itself. This aspect of DNAD was in fact addressed in some earlier literature on applications of DNAD [43,44]. But in recent years many studies are reporting the sensitivities of model parameters computed by DNAD without appropriately addressing the issue of them being a function of numerical scheme, the grid and the level of iteration convergence $[31,32,33,34,45]$. This omission, along with the use of terminology of 'exact derivatives,' gives the false impression that the computed sensitivities are highly accurate and can be used in subsequent analyses which leads to drawing of overarching conclusions. In reality, however, if the solution itself is not grid convergent or is not obtained using an accurate numerical scheme, the computed sensitivities may also incur significant errors. If used in applications such as design optimization and parameter estimation, these erroneous sensitivities could lead to faulty conclusions.

The objective of this study is to demonstrate clearly that the derivatives obtained from DNAD enabled computer codes are prone to numerical errors inherent to computational modeling. For this purpose a simple transport equation is formulated with appropriate boundary conditions such that it could be solved analytically. The exact sensitivity of the mathematical model to the 
parameters could thus be determined by symbolically differentiating the analytical solution. The chosen model equation is also solved numerically using finite volume discretization schemes. The derivatives of the numerical solution are evaluated using both DNAD and finite difference (FD) approaches and the values are compared with the exact derivatives.

Since the fuel contaminant performance degradation model has empirical parameters which are calibrated with the experiments, the DNAD model will be used to evaluate the sensitivity of the model to its empirical parameters.

\subsection{Extension to three dimensional planar cell}

An important advantage of the solid oxide fuel cells (SOFCs) that makes this technology an appealing source of energy conversion is its fuel flexibility. Throughout the world, the coal reserves are much higher than the other fossil fuels such as petroleum and natural gas. Therefore, the utilization of gasified coal (syngas) in SOFCs has become a major research area [46]. However, the drawback of using coal syngas in SOFCs is its contaminants such as phosphorus, arsenic, zinc, sulfur, mercury, selenium, and vanadium which are shown to form secondary phases with $\mathrm{Ni}$ inside SOFC anode such that the performance and durability of SOFCs diminish gradually during cell operation [6]. SOFC performance and durability during the exposure to phospine have been studied by several research groups $[6,12,16,17,18,19,20,24,46]$. Most of these studies report investigations made using button cells. Cayan et al. [12] developed a 1D phenomenological model to predict the performance and durability of button SOFCs under variety of operation conditions with the effect of different fuel contaminants including phosphine. The performance degradation calculations showed good agreement with the experimental studies. Even though button cell testing is a convenient technique for experimental studies, extrapolation of button cell result to practical 
design of planar cells may not be straight forward. Separate testing of planar cells is thus essential after initial screenings are performed with button cells. Numerical models are usually used for planar cell investigations which could provide accurate predictions and reduce the need for expensive experiments. A number of studies are available in literature on planar SOFCs modeling. Most of these studies focus on performance calculations of planar SOFCs [47-52]. However, performance of planar SOFCs due to fuel (syngas) contaminants have not been reported in the literature yet.

In this work, an in-house detailed three-dimensional computational model, DREAM SOFC $[48,53,47]$, is integrated with an in-house detailed one-dimensional computational model, [12], to simulate coupled transport processes, (i.e. heat, species, electric charge) and phosphine induced performance degradation of planar SOFC. The empirical model parameters in degradation model are calibrated with the one dimensional model [12] using button cell degradation experiments. The calibrated parameters from the one dimensional model are then used to perform simulations which predict fuel contaminant performance degradation of a planar cell. The implemented code takes into account the transport of fuel and oxidizer, chemical kinetics of hydrogen oxidation reaction catalyzed by nickel and formation of phosphine coverage which depletes the available nickel active sites and causes the performance degradation of the planar SOFCs. DREAM SOFC predicts the temperature and time dependent properties inside the SOFC, such as electrical potential, current density, porosity, coverage and spatial distribution of species inside anode and cathode. DREAM SOFC is verified and validated against benchmark problems and button cell experiments $[48,53]$. The details of the computational model are given in the numerical model section. Interested readers are referred to $[47,48,53]$ and $[6,12]$ for the detailed information about DREAM SOFC and onedimensional degradation model, respectively. 


\subsection{Objective}

The objective of this study is to use experimentally observed phenomena that are induced by fuel contaminants during the operation of anode supported SOFCs under a variety of operating conditions to predict durability and over-all gradual performance degradation of cell employing a model developed by Cayan et al. [12]. The model will be further refined, validated and extended to planar SOFC's.

The one dimensional model by Cayan et al. is refined to calculate the effect of steam concentration with the guidance of experimental observations. The current model is integrated with a supplementary impedance model to evaluate the overall cell performance during gradual degradation of the cell. Sensitivity analysis calculations with DNAD are performed to evaluate the sensitivity of the predictions to the empirical parameters involved. Then, the degradation model is integrated into the 3-D transient planar SOFC code DREAM-SOFC [54,55]. The threedimensional model which is modeled with the same physics will be used to verify PNNL experiment channel data and the phosphine induced degradation pattern in planar SOFC. Furthermore, a novel model is developed to investigate the nickel migration phenomena from anode/electrolyte interface to anode/fuel channel interface. These developments and applications are discussed further in the following sections. 


\section{CHAPTER 3 - One dimensional model and polarization, impedance analysis}

This work was presented and published in Fundamentals \& Development of Fuel Cells Conference FDFC2013, 16-18 April Karlsruhe, Germany [46].

\subsection{Background: Chapter 3}

The one-dimensional degradation model [12] solves for the distributions of gas phase specie concentrations, specie coverage on active sites and the electrical potential along the anode thickness. It is postulated that the impurity coverage on Ni sites marks the inception of performance degradation since it leads to subsequent formation of secondary phases with $\mathrm{Ni}$, thus altering the porosity, conductivity and the electrochemical activity of the anode. The effect on the porosity is modeled based on the experimental observations which show that the secondary phases block the pores. Reduced porosity could decrease the effective diffusivity of the fuel, thus the mass transport losses could increase due to the slow transport of the fuel to the active layer where electro chemical reactions occur. Although the electrical conductivity is also modeled as a function of the coverage, it is observed that the electrical conductivity does not have a significant effect on the performance degradation. In order to simulate the experimentally observed effect of steam concentration, the available model is modified using experiments as a guide. The experiments show that the steam in the fuel accelerates the $\mathrm{PH}_{3}$ induced degradation process and the formation of secondary phases. Based on this observation, the model is modified such that the variation of porosity due to the specie coverage is higher when steam is added to the fuel. The second modification is to the 
exchange current relation which is revised to make exchange current density a function of steam concentration as well based on available studies which show that the exchange current density of hydrogen is a function of the steam concentration. More details about the model will be discussed in later sections.

A supplementary computational model was integrated into the degradation model to evaluate performance of the cell at different instants during the gradual performance loss by simulating the V-I behavior and the impedance response of the cell using the instant states of anode supported SOFCs during performance degradation. These calculations are based on a cell level model which includes anode, electrolyte and cathode components of the button cell. The charge transfer inside composite electrodes is assumed to be occurring through the thickness of the electrode. The model treats each individual phase inside the composite electrolyte (electron conducting phase, ion conducting phase and pore phase) as separate continuums which are all mixed together and interact with each other at the local level through electrochemical reactions. The impedance model was

originally developed by Pakalapati et al. [56]. The model treatment and assumptions are similar to those used in similar studies from the literature [28,57]. Briefly in this model, three charge transport equations are solved: one for the electrolyte phase and one each for the electron conducting phases in the composite electrodes. Two mass transport equations are solved for concentration distributions of hydrogen and oxygen inside the anode and cathode, respectively. More details about the model will be discussed in subsequent sections.

\subsection{Degradation model}

The one dimensional computational model is developed to predict fuel contaminant induced performance degradation for SOFC anodes. This model is a refined/modified version of the previous model developed by Cayan et al. [12].The computational domain includes the thickness 
of the anode as shown in Figure 3.1a. As it is elucidated in Figure 3.1b, porosity, exchange current density and electrical conductivity are functions of the local contaminant coverage. It should be noted here that the electrochemical reactions are assumed to be occurring only at the anode/electrolyte interface and hence the exchange current is a function of contaminant coverage at this interface.
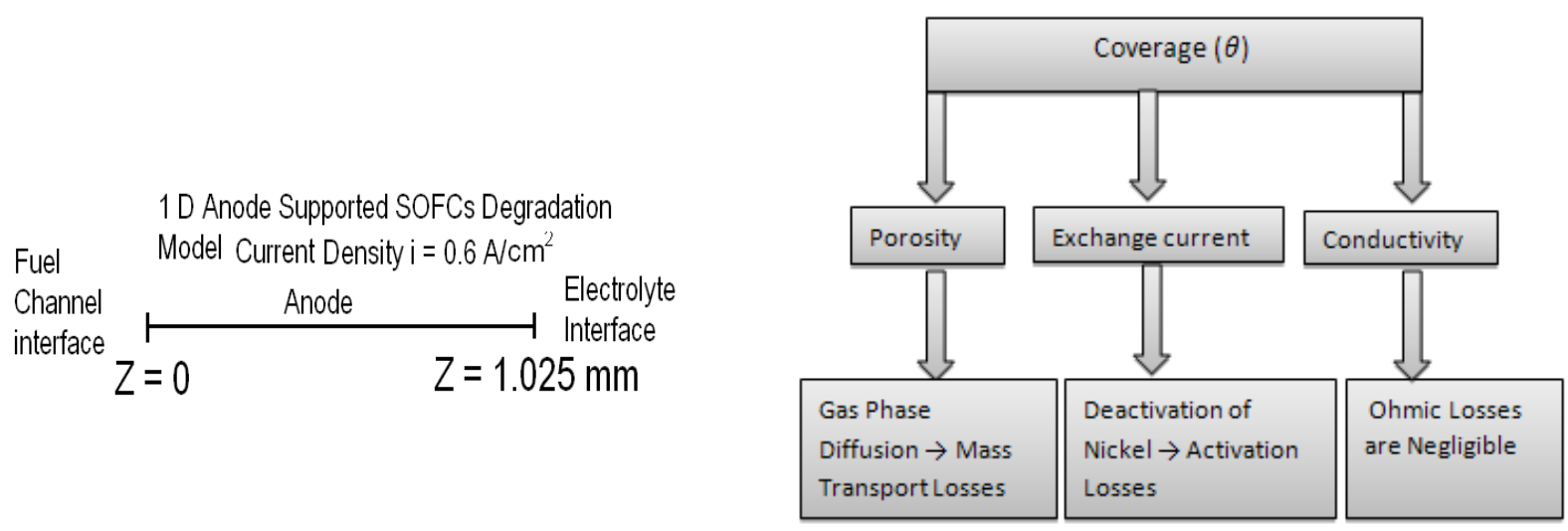

Figure 3.1: (a) 1D Anode supported SOFC (b) Model dependency to contaminant's coverage

While the original model [12] was successfully applied to predict performance degradation of SOFCs due to several fuel contaminants under different operating conditions, the experimental results [19] regarding the effect of steam on $\mathrm{PH}_{3}$ induced degradation were not readily reproduced by it. Particularly the model predictions matched well with one set of experimental data where the fuel is humidified ('wet fuel' case), the agreement was poor for the second set where no steam was added to the fuel ('dry fuel' case). Revision of the model equations is thus deemed necessary to account for the influence of steam concentration on degradation. From the experimental observations and Ellingham diagrams the presence of the steam seems to accelerate the formation of secondary phases $[19,20]$. Therefore, the functional dependence of the porosity on contaminant 
coverage for the dry fuel case is recalibrated separately using experimental results. Accordingly, Eq. 3.1 shows the calibrated functional dependency of porosity on the contaminant coverage.

$$
\varepsilon=\varepsilon_{0}\left(1-0.95 \theta^{p}\right)
$$

Where $\varepsilon_{0}, \varepsilon$, and $\theta$ are initial porosity, porosity, and contaminant coverage, respectively. It has been noted in the literature [25,26,27] that the exchange current density of hydrogen varies with the steam concentration and based on experimental observations at NIFT [15,20], it is observed that the Ni-P secondary phases formed under wet fuel conditions have a more profound effect on the kinetic properties of the anode than those formed under dry fuel conditions. As a consequence of these findings, the effect of steam concentration on hydrogen exchange current density is incorporated by modifying the exchange current density as follows:

$$
i_{0, H_{2}}=C_{1} y_{H_{2} O}^{3 / 4}\left(\frac{y_{H_{2}}}{y_{H_{2}, r e f}}\right) \exp \left(\frac{-E_{H_{2}, a c t}}{R_{u} T}\right)\left(1-\theta_{P H_{3}}^{m}\right)^{n}
$$

In eq. (2), $m$ and $n$ are the model parameters obtained from the available experimental data. The constant $C_{1}$ is $3.936 \times 10^{7}$, the hydrogen reference concentration, $y_{H_{2}}$,ref , is 0.333 , the hydrogen activation barrier, $E_{\mathrm{H}_{2}}$,act is $91.661 \mathrm{~kJ} / \mathrm{mol}$ [6]. The contaminant coverage can affect conductivity and hence the ohmic losses. However, it is postulated that the new phases which are the resultant products of contaminant attack have more or less the same electrical conductivity as nickel itself. Therefore, the ohmic losses due to secondary phase formation are assumed to be negligible. 


\subsection{Polarization and impedance model}

The supplementary numerical model used in this study is a cell level model which includes anode, electrolyte and cathode components of the button cell. It was originally developed by Pakalapati et al. [56] at the National Institute of Fuel Cell Technology (NIFT) center, WVU. The charge transfer inside composite electrodes is assumed to be occurring through the thickness of the electrode. The model treats each individual phase inside the composite electrolyte, viz electron conducting phase, ion conducting phase, and pore phase as separate continuums which are all mixed together and interact with each other at local level through electrochemical reactions. The model treatment and assumptions are similar to those used in similar studies from literature $[27,28]$.The electrolyte phase extends the entire computational domain wherein it is distributed as a well percolated porous network inside the composite electrode regions and is the exclusive phase inside the bulk electrolyte region. The anode and cathode on either side of the bulk electrolyte are composite regions which consist of an electron conducting phase and a pore phase in addition to the electrolyte. Three charge transport equations are solved: one for the electrolyte phase and one each for the electron conducting phases in composite electrodes. Two mass transport equations are solved for concentration distributions of hydrogen and oxygen inside the pore phase of the anode and cathode respectively. The charge transport equations for the electron and ion conducting phases in composite electrodes are given by Eqs. (3.3) and (3.4) respectively:

$$
\begin{aligned}
& a_{i n t} C_{D L} \frac{\partial}{\partial t}\left(\varphi_{e}-\varphi_{i}\right)+\nabla \cdot\left(-\sigma_{e} \nabla \varphi_{e}\right)=i_{F} \\
& -a_{i n t} C_{D L} \frac{\partial}{\partial t}\left(\varphi_{e}-\varphi_{i}\right)+\nabla \cdot\left(-\sigma_{i} \nabla \varphi_{i}\right)=-i_{F} \\
& \nabla \cdot\left(-\sigma_{i} \nabla \varphi_{i}\right)=0
\end{aligned}
$$


Here, $\varphi$ is the electric potential, $i_{F}$ is the Faradic current, $a_{i n t}$ is the specific interface area between the ion conducting and electron conducting phases, $C_{D L}$ is the double layer capacitance and subscripts ' $e$ ' and ' $i$ ' stand for electronic and ionic respectively. The first term in eqs (3.3) and (3.4) represents the charging of the double layer at the active interface. In the bulk electrolyte both double layer charging and faradaic currents disappear and the charge conservation reduces to eq. (3.5). The faradaic current is treated as a function of local over-potential. In lieu of a detailed elementary reaction mechanism to describe the electrochemistry, a simple global Butler-Volmer type rate equation is used to model the charge transfer across the electrode and electrolyte phases. The equations used for cathode and anode are Eqs. (3.6) and (3.7), respectively.

$$
\begin{gathered}
i_{F, c}=i_{0, O_{2}} \frac{\left(p_{O_{2}} / p_{O_{2}, *}\right)^{1 / 4}}{1+\left(p_{O_{2}} / p_{O_{2}, *}\right)^{1 / 2}} \times\left\{\frac{p_{O_{2}}}{p_{O_{2}, \text { in }}} \exp \left(\frac{0.5 F \eta_{a c t, c}}{R T}-\right)-\exp \left(-\frac{0.5 F \eta_{a c t, c}}{R T}\right)\right\} \\
i_{F, a}=i_{0, H_{2}}\left(p_{H_{2}}\right)^{1 / 4}\left(p_{H_{2} O}\right)^{1 / 2} \\
\times\left\{\frac{p_{H_{2}}}{p_{H_{2}, i n}} \exp \left(\frac{0.5 F \eta_{a c t, a}}{R T}-\right)-\frac{p_{H_{2} O}}{p_{H_{2} O, i n}} \exp \left(-\frac{0.5 F \eta_{a c t, a}}{R T}\right)\right\}
\end{gathered}
$$

Here $\eta_{c}$ and $\eta_{a}$ are local over-potentials and $i_{F, c}$ and $i_{F, a}$ the corresponding volumetric charge transfer rates inside cathode and anode, respectively. The partial pressure of the specie ' $i$ ' is denoted by $p_{i}$ and subscript ' $i n$ ' is used for inlet partial pressures. The model parameters are $i_{0, O_{2}}, i_{0, H_{2}}$, and $p_{O_{2}, *}$ Equations (3.6) and (3.7) are adopted from work of Zhu and Kee [27] and Bessler [57] 2006 respectively, by multiplying the exponential terms with normalized local concentrations of the reactants to explicitly add the concentration over-potential. Also here, the charge transfer rates are interpreted on per unit volume basis as was done by Bessler et al. [57] and thus the units of model parameters $i_{0, O_{2}}$, and $i_{0, H_{2}}$, accordingly modified and numerical values are higher. The 
reader is referred to [29] for more details of the model. The species transport equation solved for gas concentrations in pore phases of the anode and cathode is given by:

$$
\frac{\partial \varepsilon \phi}{\partial t}=\nabla \cdot\left(D_{e f f} \nabla \phi_{e}\right)+S_{\phi}
$$

Here $\phi$ is the specie concentration, $\varepsilon$ is the porosity, $D_{\text {eff }}$ is the effective diffusion coefficient of the specie in the electrode pores and $S_{\phi}$ is the source of the specie due to electrochemical equations. With appropriate boundary conditions Eqs. (3.3-3.8) are a closed set of equations that will give distributions of electric potential, charge transfer, and specie concentrations inside the button cell. Polarization curves are obtained by repeating the simulations for different applied voltages. For impedance simulations, a sinusoidal voltage signal is given as an input and the output current signal is used to determine the impedance at that frequency. The procedure is repeated for a range of frequencies to obtain the complete impedance curve.

Even though the original model allows for charge transfer to occur away from the active interface, this aspect is disabled for the simulations presented in this study and the charge transfer reaction is limited to the electrode/bulk electrolyte interfaces. This was done in order to be consistent with the electrochemistry model used in the code that predicts the cell degradation. After this modification, the parameters in the model are calibrated to obtain reasonable agreement with measured polarization and impedance curves of a real button cell $[29,58]$. The corresponding parameters in the degradation model are matched with the calibrated parameters and it is verified that the simulated anode polarization from the degradation model at zero time matched that from the button cell code. After this the predicted distributions of anode porosity and anode conductivity at different times obtained from the degradation code are input to the button cell code and the 
polarization and impedance curves at that stage are predicted following the procedure described above.

\subsection{Results: Chapter 3}

The model described above is used to simulate the effect of steam on the performance degradation of anode supported button SOFC due to the $\mathrm{PH}_{3}$ exposure. As it can be seen from the experimental degradation curve (Figure 3.2 a), the phosphine degradation is less severe in the absence of steam. Here, time zero corresponds to the time at which the $\mathrm{PH}_{3}$ injection is started. The parameters (Table 3.1) were thus calibrated separately for these two cases and the only difference between the two cases is the value of parameter ' $p$ ' in porosity equation (Eq. 3.1).

Simulations were performed for button cells with $1025 \mu \mathrm{m}$ thick anode, operating under $10 \mathrm{ppm}$ of PH3 contamination, at a constant current density of $0.6 \mathrm{~A} / \mathrm{cm}^{2}$ and fuel concentrations of $\quad 97 \%$ $\mathrm{H}_{2}, 3 \% \mathrm{H}_{2} \mathrm{O}$, and $100 \% \mathrm{H}_{2}, 0 \% \mathrm{H}_{2} \mathrm{O}$ for wet and dry cases respectively. With the new modifications to the model, reasonable agreement was achieved between the simulations and the experimental results (Figure 3.2 a) in terms of the effect of steam on degradation due to phosphine. Figure $3.2 \mathrm{~b}$ shows the losses during the gradual degradation. 
Table 3.1:Model parameters: $\boldsymbol{k}_{\boldsymbol{f}, \boldsymbol{x}}, \boldsymbol{k}_{\boldsymbol{b}, \boldsymbol{x}}$ are forward and backward reaction rate of species x respectively, $\mathrm{m}, \mathrm{n}, \mathrm{p}, \mathrm{q}$ are model parameters.

\begin{tabular}{cc}
\hline Parameter & $\mathbf{1 0} \mathbf{~ p p m}$ \\
\hline Temperature, ${ }^{\mathbf{0}}$ & 800 \\
${\text { Current density, } \mathbf{A} / \mathbf{c m}^{2}}_{\boldsymbol{k}_{\boldsymbol{f}, \boldsymbol{x}}, \mathbf{s}^{-1}}$ & 0.6 \\
$\boldsymbol{k}_{\boldsymbol{b}, \boldsymbol{x}}, \mathbf{s}^{-\mathbf{1}}$ & $5.0 \times 10^{2}$ \\
$\mathbf{m}$ & $9.1 \times 10^{-3} \exp \left(-9.23 \times 10^{4} / \mathrm{RT}\right)$ \\
$\mathbf{n}$ & 2.0 \\
$\mathbf{p}$ & 7.0 \\
$\mathbf{q}$ & Dry $\left(\% 0 \mathrm{H}_{2} \mathrm{O}\right): 0.5$, Wet $\left(\% 3 \mathrm{H}_{2} \mathrm{O}\right): 2.0$ \\
\hline
\end{tabular}

It can be seen from the simulated degradation curves that initially the degradation process is due to the mass transport and activation losses; however, the subsequent sharp performance losses are predominantly due to the activation losses (Figure $3.2 \mathrm{a} \& b$ ). Figure $3.3 \mathrm{a} \& \mathrm{~b}$ show the normalized activation losses and the contaminant coverage at the active interface as function of time. It is seen in Figure 3.3 that sharp increase in losses occurs once the contaminant coverage reaches the active layer (Figure $3.3 \mathrm{~b}$ ), as it deactivates the available nickel sites. Though the conductivity has a functional dependency on contaminant coverage as it was shown on Figure $3.1 \mathrm{~b}$, the losses due to the electrical conductivity are negligible which can be seen in Figure $3.2 \mathrm{~b}$.

The variation of normalized coverage and porosity distributions along the anode thickness at different exposure times are depicted on Figure $3.4 \mathrm{a} \& \mathrm{~b}$, respectively. The strong functional dependency of porosity on the coverage can be observed from Figure $3.4 \mathrm{a} \& \mathrm{~b}$. It is seen that as the trace element coverage steadily increases over the time and it penetrates from anode surface towards the active interface, the porosity decreases accordingly, thus mass transport losses increase. Figure $3.3 \mathrm{~b}$ shows the variation of phosphine-coverage in the active layer with time, this trend can also be extracted from Figure 3.4 at $\mathrm{z}=1.025 \mathrm{~mm}$. 

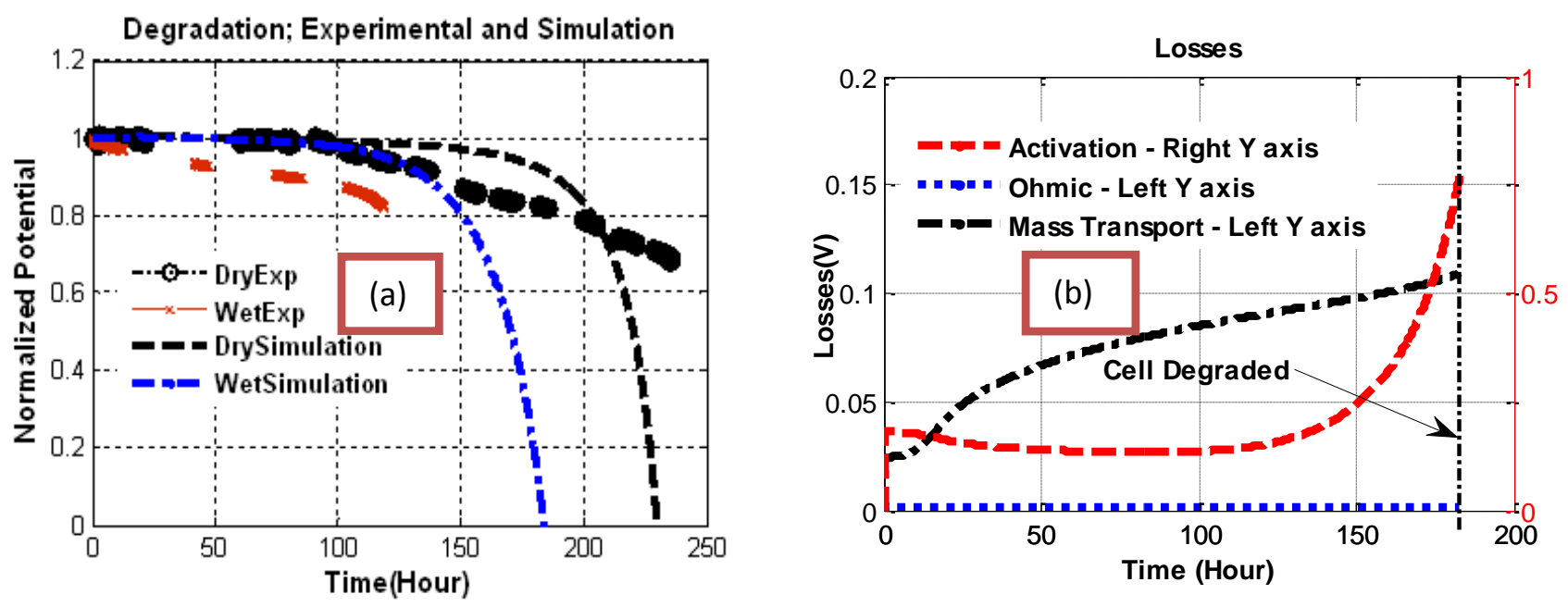

Figure 3.2: (a) Performance degradation due to $10 \mathrm{ppm} \mathrm{PH}_{3}$; comparison of computational model with experimental results for wet and dry fuel cases. (b) Losses during gradual degradation for wet fuel case predicted by the degradation model.

The V-I behavior and impedance response computational model is used to analyze a different cell with a relatively thinner anode $(250 \mu \mathrm{m})$. This was done since the base line impedance and polarization data for the cells used in experiments of [19] were not available. Thus, a cell with known baseline performance data is chosen and the parameters in the supplementary model are calibrated to match the experimental performance data. The corresponding model parameters in the anode degradation model are matched with the calibrated parameters of the supplementary model and it was verified that the predicted baseline anode performance is the same for these two models. The degradation of this second anode geometry is then simulated with the same functional dependence of cell properties on coverages that are given in Table 3.1 for wet fuel case. In order to assess overall performance of the cell during the gradual degradation, the predicted porosity profiles along the anode at different contaminant exposure times and the corresponding instantaneous exchange current density of the cell are used as input values to simulate V-I behavior and the impedance response of the cell at those instants. Electronic/ionic conductivity was kept 
constant during the performance degradation simulation due to its negligible effect on degradation process.

Figure 3.5 shows the predicted degradation curve for the second anode geometry under wet fuel conditions. Simulations (Figure $3.5 \mathrm{~b}$ ) with lower levels of $\mathrm{PH}_{3}(2 \mathrm{ppm} \& 5 \mathrm{ppm})$ were performed with the same parameters that are listed in Table 3.1. As expected; the contaminant level has a significant effect on the performance losses with lower concentrations causing slower degradation rates. The V-I behavior and the impedance response of cell simulated different degradation times are shown in (Figure $3.6 \mathrm{a} \& \mathrm{~b}$ ), respectively. The V-I curves obtained at different points on the degradation curve (Figure 3.5 a) clearly show that while $\mathrm{PH}_{3}$ coverage increases (Figure 3.4 a), the losses increase, slowly at first and sharply after some time. The impedance curves show the predicted impedance response of anode supported cell while anode is exposed to phosphine over time. In these impedance curves the smaller arc corresponds to the cathode polarization and the large arc is due the anode polarization. It is seen from the impedance curves that the increase in activation losses in the anode is mainly responsible for cell degradation and they increase abruptly after $8 \mathrm{~h}$ of exposure time. The impedance curve for 12 hour exposure time is not shown since the anode arc for that case is too large. Additionally Figure 3.6 a, also shows that the initial changes in V-I curves with exposure time were mostly due to increase in activation losses since the slope of V-I at low over-potentials is increasing. These observations are consistent with those made for the thicker anode geometry (Figure $3.3 \mathrm{~b}$ ) where it is shown the sharp increase in activation losses are mostly responsible for cell failure. Also the concentration losses seem to be even less of a factor in the performance degradation for the second anode geometry (Figure 3.6 a) since the anode thickness is significantly smaller. 

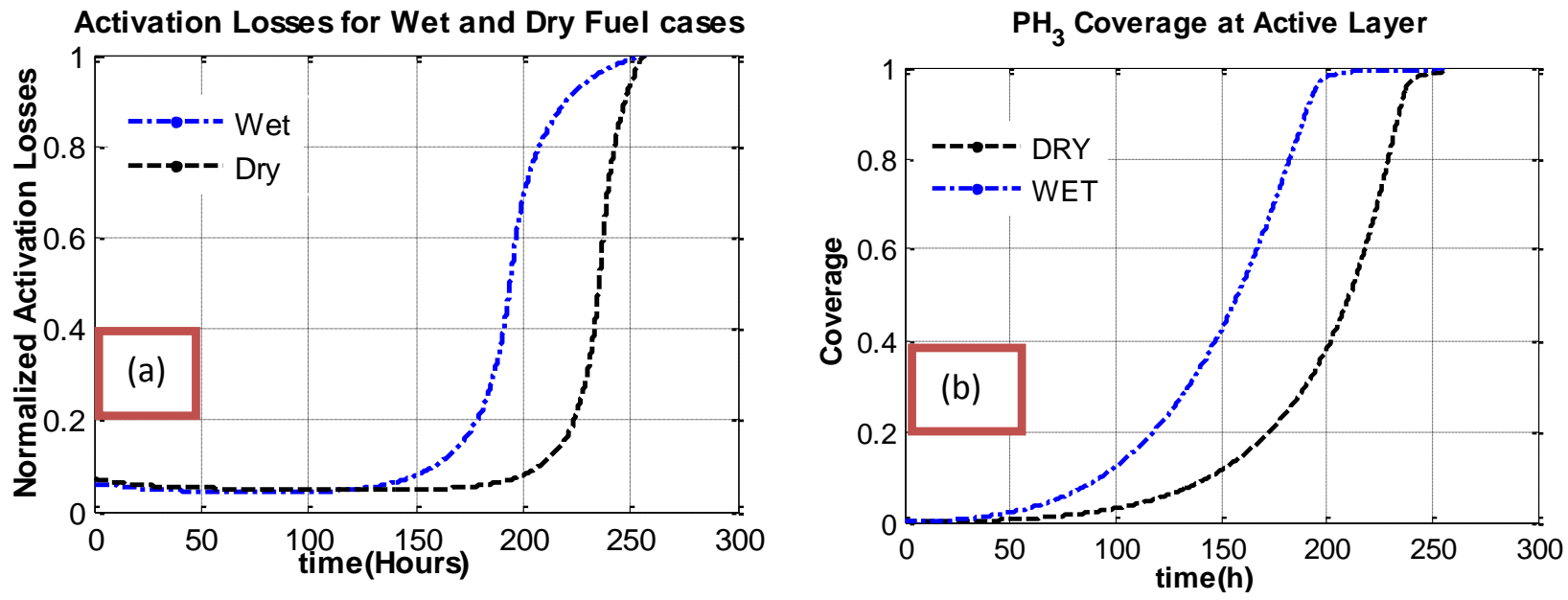

Figure 3.3: (a) Normalized activation losses for wet and dry fuel cases (b) $\mathrm{PH}_{3}$ coverage at active layer for wet and dry cases
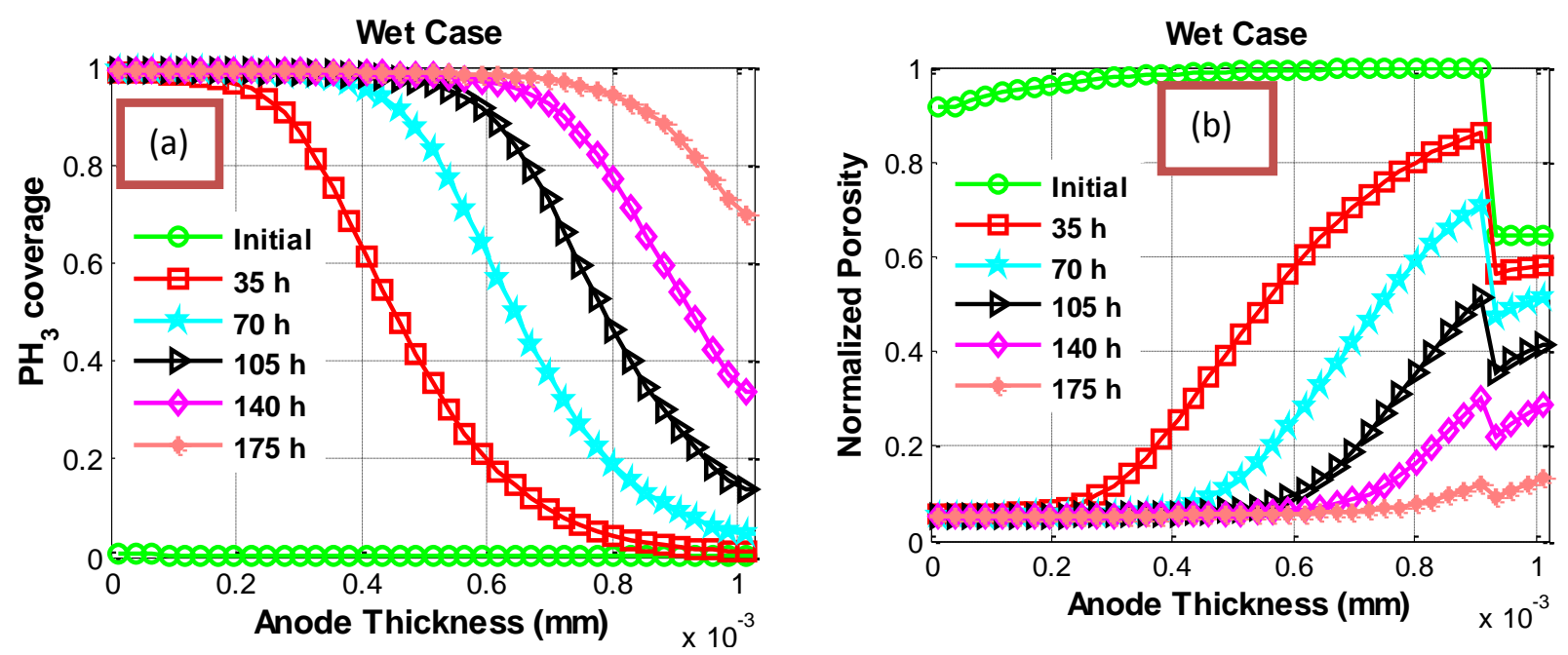

Figure 3.4: (a) Normalized $\mathrm{PH}_{3}$ coverage profile along the anode during gradual degradation time for wet fuel case. (b) Normalized porosity profile along the anode during the gradual degradation time for wet fuel case. 

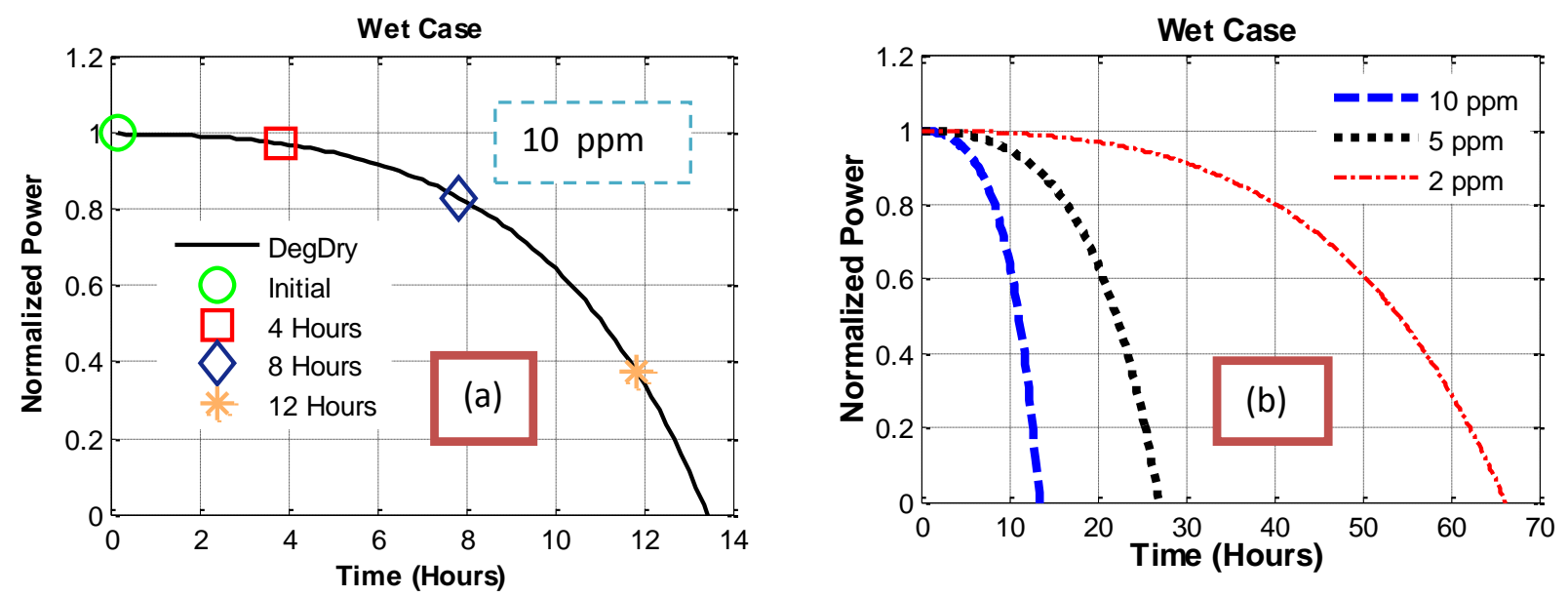

Figure 3.5: Predicted performance degradation for wet fuel case; performed for SOFCs with 250 $\mu \mathrm{m}$ thick anode exposed to (a) $10 \mathrm{ppm} \mathrm{PH}_{3}$ pointed for V-I and Impedance simulations (b) 10 ppm, 5 ppm and 2 ppm $\mathrm{PH}_{3}$
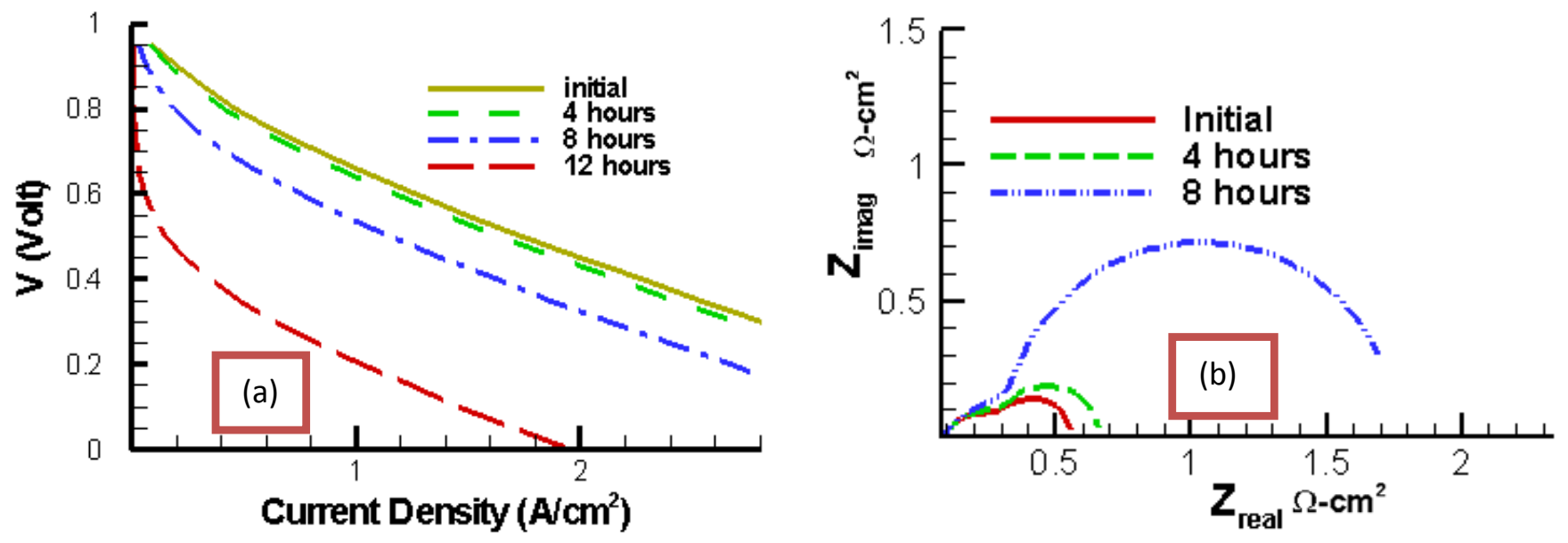

Figure 3.6: Predicted V-I (a) and impedance (b) curves at different times of phosphine exposure for wet hydrogen.

\subsection{Conclusions: Chapter 3}

An in house 1D performance degradation model is refined and then used to investigate the $\mathrm{PH}_{3}$ poisoning in the anode of SOFCs under different steam concentrations by examining the transport and reactions both in the gas phase and in the solid phase. The model is modified using the experiments conducted at NIFT, WVU as a guide to simulate the effect of steam concentration. 
Model parameters are calibrated according to experiments. The calibrated model was used to estimate performance degradation for both wet and dry fuel case exposure to $10 \mathrm{ppm} \mathrm{PH}_{3}$ with $1025 \mu \mathrm{m}$ and $250 \mu \mathrm{m}$ thick anodes. Good agreement was achieved between model and available experimental data. A supplementary computational model was integrated to the degradation model to evaluate performance of the cell at different times of the gradual performance loss, by simulating the V-I behavior and the impedance response of the cell using the instantaneous states of gradual anode degradation process. Simulations were also performed under lower level impurity concentrations. The results that are obtained with low level $\mathrm{PH}_{3}$ concentrations show expected trends with lower concentrations causing slower degradation rates. 


\section{CHAPTER 4 - Sensitivity analysis of the model parameters}

Part of this work was presented and published in Proceedings of the ASME 2013 Fluids

Engineering Summer Meeting FEDSM2013, July 7-11, 2013 [59]

\subsection{Introduction}

Sensitivity analysis of a computational model to any existing model parameter is the derivative of the model with respect to that parameter. In experimental works, evaluating the sensitivity of the experiment to the parameters involved is crucial for the accuracy of the results. In this study, the models that are proposed are empirically developed with the model parameters which are calibrated using experiments. In order to investigate the dependency of the model predictions on its parameters, a sensitivity analysis is performed by using Dual Numbers Automatic Differentiation (DNAD) and Finite Difference (FD) approach.

The DNAD method uses Dual number arithmetic to calculate the derivative of any mathematical functions or any numerical model. Dual number arithmetic is a well-known strategy [59] for automatic differentiation of computer codes which gives exact derivatives, to the machine accuracy, of the computed quantities with respect to any of the involved variables. The sensitivity to a model parameter calculated by DNAD is essentially that of the combination of the actual mathematical equations, the numerical scheme and the grid used to solve the equations, not that of the model equations alone as implied by some studies. This aspect of the sensitivity analysis of numerical simulations using DNAD is explored in this study by a simple one-dimensional 
convection- diffusion equation which is discretized using different schemes of finite volume method. Derivatives of the numerical solutions with respect to parameters are evaluated automatically using DNAD and finite differencing. The analytical solution was also found for the original PDE and derivatives of the true solutions are computed analytically to prove the power of DNAD method for sensitivity analysis. After validating this procedure it is further applied to the contaminant degradation model presented in chapter 3.

\subsection{Mathematical model}

The model equation whose sensitivity to the parametric constants is analyzed in this study is given by:

$$
u \phi_{x}=\Gamma \phi_{x x}+C \phi
$$

Equation (4.1) is chosen because it represents the kernel of any CFD model, the governing equation for transport of a generic scalar; albeit in a simplified form applicable to one-dimensional steady state problems. Accordingly, $\phi$ is the generic scalar, $u$ is the velocity, $\Gamma$ is the diffusivity of the scalar and the source term for the scalar is linearly proportional to the scalar value with $C$ being the proportionality constant. Here $u$, $\Gamma$ and $C$ are treated as parametric constants for the model and the sensitivity of the model predictions to these parameters is assessed using different methods as explained below.

In order to compare and assess different schemes of sensitivity analysis, it is desirable to know the analytical or exact values of sensitivity of the model to the parameters. This is the reason for

choosing the simplified version of the transport equation given by Eq. (4.1) which can be solved 
readily given appropriate boundary conditions. The following boundary conditions are adopted and are also used in the subsequent numerical solutions.

$$
\phi(x=0, u, \Gamma, C)=1 ; \quad \phi(x=1, u, \Gamma, C)=0
$$

With the boundary conditions given in Eq. (2), the exact solution of Eq. (1) is given by:

$$
\phi(x, u, \Gamma, C)=\frac{e^{\lambda_{2}}}{e^{\lambda_{2}-e^{\lambda_{1}}}} e^{\lambda_{1} x}-\frac{e^{\lambda_{1}}}{e^{\lambda_{2}-e^{\lambda_{1}}}} e^{\lambda_{2} x}
$$

where $\lambda_{1,2}=\frac{u \pm \sqrt{u^{2}-4 \Gamma C}}{2 \Gamma}$ when $u^{2}-4 \Gamma C>0$

Equation (3) can be differentiated with respect to each of the model parameters considered here to determine the exact sensitivity of the 'mathematical' model predictions.

\subsection{Numerical method}

Finite volume method approach is used to discretize Eq.4.1 with different schemes (upwind and exponential) [60]. The discretized equation for a representative finite volume (or computational cell) is given by:

$$
a_{P} \phi_{P}=a_{E} \phi_{E}+a_{W} \phi_{W}
$$

Where the coefficients for upwind and exponential schemes are given by Eqs. (4.5) and (4.6) respectively:

$$
a_{P}=a_{E}+a_{W}, \quad a_{E}=\Gamma / \Delta x, \quad a_{W}=u+\Gamma / \Delta x
$$




$$
a_{P}=a_{E}+a_{W}, a_{E}=\frac{u}{\exp (P)-1}, a_{W}=\frac{u \exp (P)}{\exp (P)-1}, P=\frac{u \Delta x}{\Gamma}
$$

The linear system of equations given by Eq. (4) is solved using a MATLAB program. Computations are performed for different grid sizes (11, 41, and 161 nodes).

\subsection{Sensitivity estimation}

Two different methods are used to determine the derivatives of the numerical solution with respect to the parameters in the model: finite difference method and dual number automatic derivation (DNAD). In the first method, in order to estimate the derivative with respect to a single parameter, the simulation is repeated twice with two values for the parameter differing by an arbitrarily small amount while keeping all other parameters fixed. The derivative is then calculated using the finite difference formula. For example, Eq. 4.7 shows the finite difference formula for derivative with respect to $\mathrm{C}$.

$$
\frac{\partial \phi(x, u, \Gamma, C)}{\partial u}=\frac{\phi^{n}(x, u, \Gamma, C+\Delta C)-\phi^{n}(x, u,\ulcorner, C)}{\Delta C}
$$

Clearly the estimated derivatives are strongly dependent on the step size ( $\Delta C$ in Eq. 4.7) used. However, a relatively small step size of 0.001 is used for each parameter in this study to maintain accuracy. This step size is deemed to be sufficiently small to minimize the truncation error while not incurring cancellation errors due to rounding off.

Derivatives using dual numbers are obtained by modifying the MATLAB code that solves the model equations to convert all the appropriate variables to dual numbers and overload all mathematical operations involving dual numbers with dual number arithmetic. The MATLAB 
class for dual numbers developed by Taylor [61] is used for this purpose. In this elegant method for calculating sensitivities, the derivatives of numerical solution with respect to all model parameters can be determined in a single execution of the program. The errors in derivations with respect to the parameters $(u, \Gamma, C)$ are calculated for finite differences derivatives and dual numbers automatic derivatives are evaluated as the difference between the approximate derivatives and the exact analytical derivatives of the true solution given by Eq. (4.3).

\subsection{Results}

The initial values of the parameters that the sensitivities are analyzed are 1.0, 0.1 and -1.0 for $u$, $\Gamma$ and $C$, respectively. It should be first noted that DNAD does indeed give the exact derivatives, to the machine accuracy, of the actual calculations performed in the solution algorithm. This is verified here by comparing the analytical derivatives of the solution given in Eq. (4.3) with the DNAD derivatives obtained when Eq. (4.3) is used in the computer code with dual number arithmetic. It is observed that the difference between these two derivatives is the order of the machine accuracy. For comparison, the exact solution given in Eq. (4.3) is also differentiated numerically using Finite Differences (FD) and the results for DNAD and FD derivatives of the exact solution are compared in Figure 4.1-3.

Figure 4.1 shows the derivative of the exact solution with respect to the parameter $u$ obtained using FD and DNAD, along with the exact derivatives obtained by differentiating Eq. (4.3) analytically. Figure $4.1 \mathrm{~b}$ shows the error in the derivatives obtained from FD and DNAD. It can be seen that while both FD and DNAD approaches show good agreement with the exact derivatives, DNAD derivatives are identical to the exact values with error levels as low as the same as the machine accuracy. 

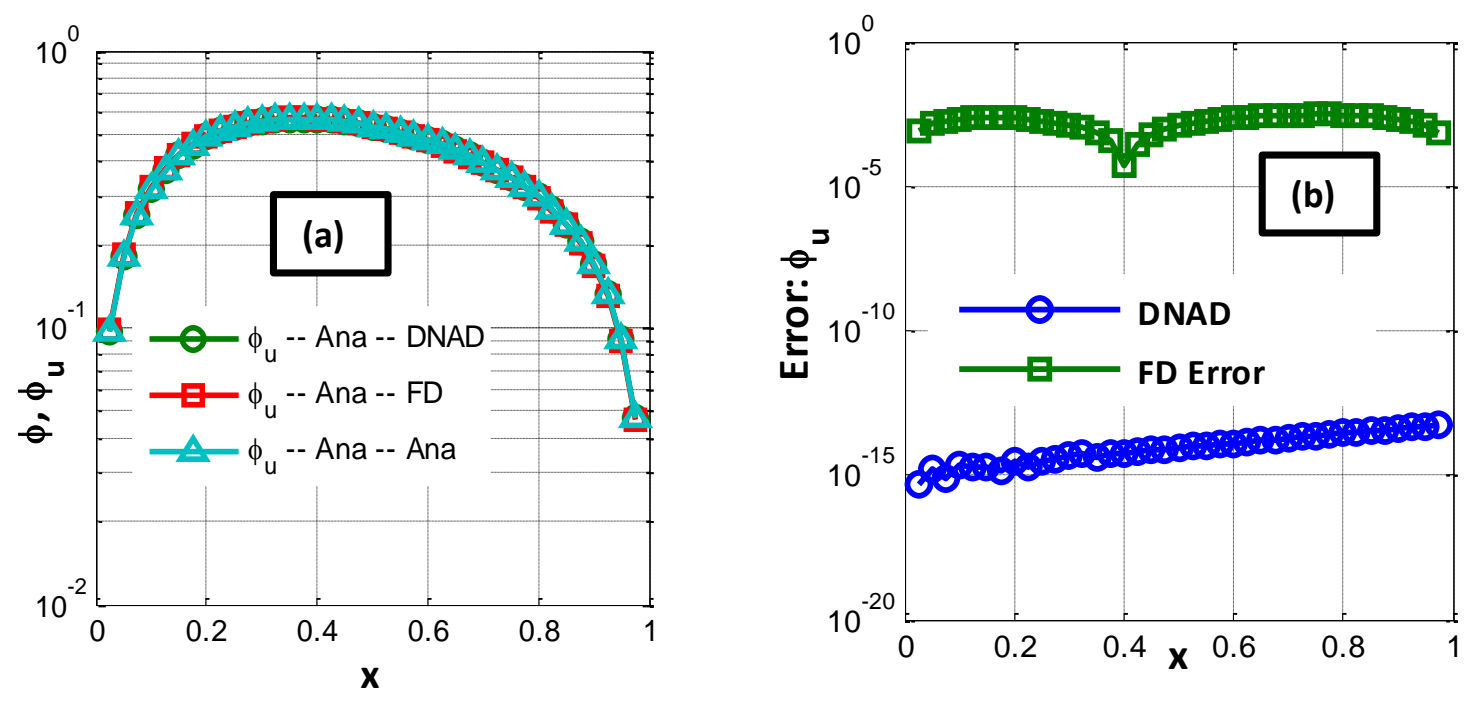

Figure 4.1: a) The first derivative of the analytic function, $\phi$, with respect to $u$ : using dual numbers (DNAD), finite differences (FD) and analytic derivative. b) the deviation of dual number (DNAD) and finite difference (FD) derivatives from analytic derivative.
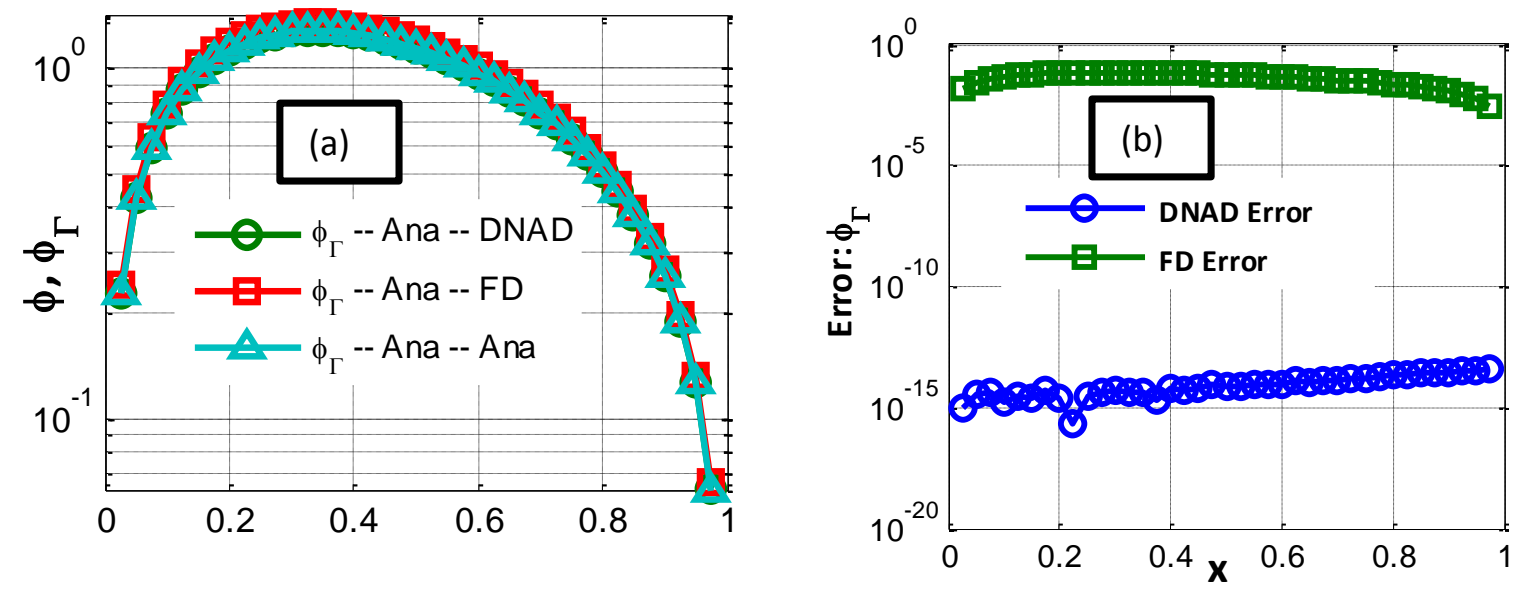

Figure 4.2: a) The first derivative of analytic function, $\phi$, with respect to $\Gamma$ : using dual numbers (DNAD), finite differences (FD) and analytic derivatives. b) The deviation of dual (DNAD) and finite difference (FD) derivatives from analytic derivative 
The error in the FD derivatives, on the other hand, is several orders of magnitude higher than that in the DNAD derivatives ( Figure 4.1b). Figure 4.2a and Figure 4.3a show that the derivatives of the analytical solution (Eq. 3) with respect to the parameters $\Gamma$ and $C$, respectively, are also estimated accurately by both FD and DNAD methods. The errors in FD and DNAD derivatives shown in Figure $4.2 \mathrm{~b}$ and Figure $4.3 \mathrm{~b}$ once again reveal that the DNAD gives nearly exact values whereas the FD approach gives only approximate values.

The results shown in Figure 4.1-4.3 show that the DNAD scheme is accurate to the machine accuracy in computing derivatives of analytical functions. However, derivatives of solutions to complex model equations, such as the species transport equations used in CFD, may not give true sensitivity of the model equations to the model parameters due to the numerical errors of the computational model. In other words, DNAD gives the derivative of the numerical solution of the model equations rather than the derivative of the exact solution. The accuracy of the numerical solution is strongly dependent on the numerical method and grid used for solving the equation. Thus it is to be expected that the sensitivities estimated using numerical solutions are also dependent on these factors. In what follows this aspect of DNAD and FD derivatives is illustrated for the numerical solution of the model. 

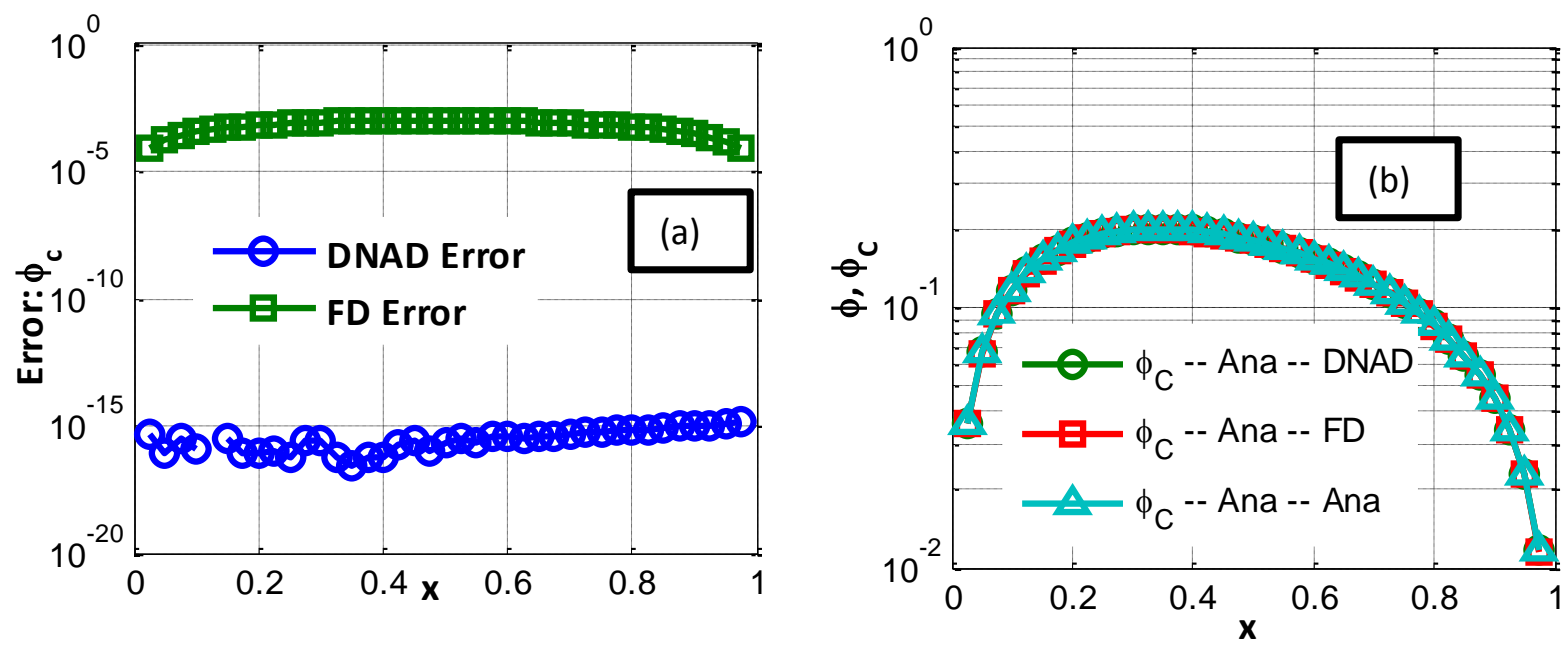

Figure 4.3: a) The first derivation of analytic function, $\boldsymbol{\phi}$, with respect to $\boldsymbol{C}$ : using dual numbers (DNAD), finite differences (FD) and analytic function. b) The deviation of dual numbers (DNAD) and finite differences (FD) derivatives from analytic derivative
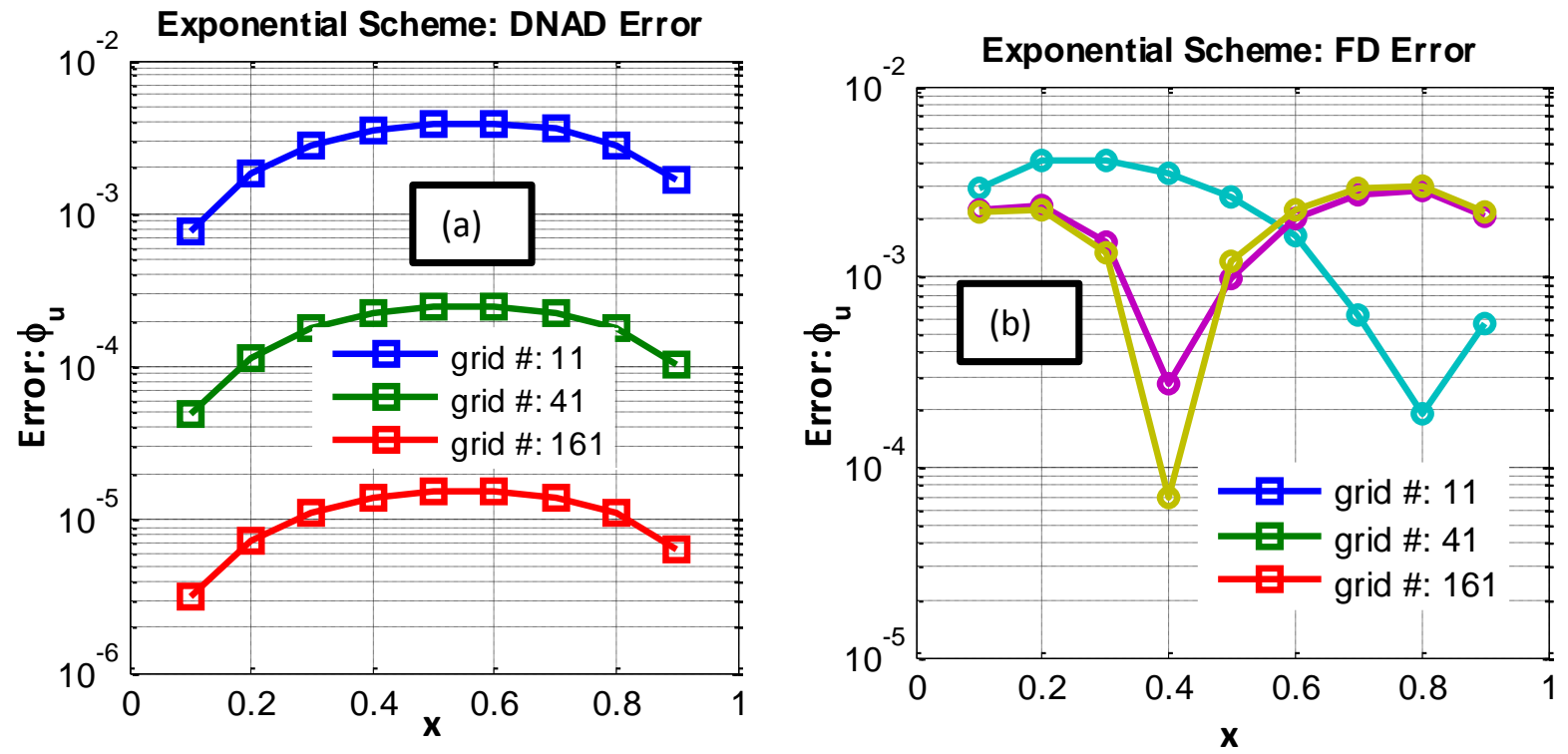

Figure 4.4: Error in derivative with respect to $\boldsymbol{u}$ of the numerical solution on different grids using finite volume method with exponential scheme: a) Error in the Dual numbers (DNAD), b) Error in the finite differences (FD) 
Figure 4.4 shows the error in the derivative of the solution with respect to parameter ' $u$ ' estimated by DNAD and FD schemes using three different grids. In these simulations, the exponential scheme is used for discretization of the model equation. The accuracy of derivatives estimated by DNAD seems to improve as the number of grid nodes is increased (Figure 4.4a). Consequently, exact derivatives of the numerical solution estimated by DNAD approach the analytical derivate of the real solution as the grid is refined. The derivative from FD method, however, showed improvement only between 11 and 41 grid nodes.

\subsubsection{Application to contaminant performance degradation model}

The DNAD, sensitivity analysis is implemented to a one-dimensional fuel contaminant performance degradation model of SOFCs anode with the parameters that are given on Table 4.1. The flow chart of the model is depicted in Figure 4.5. The transport equations (species transport, electrical potential, and contaminant coverage) in this model (Figure 4.5) are solved numerically by using finite volume discretization approach. Again two different methods are used to determine the derivatives of the numerical solution with respect to the parameters in the model: finite difference method and dual number automatic derivation (DNAD), method.

Since the one-dimensional performance degradation model is originally written in FORTRAN, the sensitivity using dual numbers is obtained by converting all the appropriate variables into dual numbers inside the FORTRAN code. In addition, the FORTRAN module for dual numbers, AUTO_DERIV [41], is implemented into the original one-dimensional performance degradation code. AUTO_DERIV is capable of overloading all mathematical operations involving dual numbers with dual number arithmetic. 
Table 4.1: Model parameters and cell operating conditions

\begin{tabular}{|c|c|}
\hline Temperature, ${ }^{\circ} \mathrm{C}$ & 800 \\
\hline Current density, $\mathbf{A} / \mathbf{c m}^{2}$ & 0.1 \\
\hline$k_{f, x}, \mathrm{~s}^{-1}$ & $5.0 \times 10^{2}$ \\
\hline$k_{b, x}, \mathrm{~s}^{-1}$ & $9.1 \times 10^{-3} \exp \left(-9.23 \times 10^{4} / \mathrm{RT}\right)$ \\
\hline $\mathbf{m}$ & 2.0 \\
\hline $\mathbf{n}$ & 7.0 \\
\hline $\mathbf{p}$ & 0.5 \\
\hline q & 2.0 \\
\hline Anode thickness $(\mu \mathrm{m})$ & 50 \\
\hline Fuel composition & $\% 99.99 \mathrm{H}_{2}, 1 \mathrm{e}-4 \mathrm{H}_{2} \mathrm{O}, 10 \mathrm{ppm} \mathrm{PH}_{3}$ \\
\hline
\end{tabular}

Using the FORTRAN module, AUTO_DERIV, the derivatives of numerical solution with respect to all model parameters can be determined in a single execution of the program. The derivatives with respect to the parameters ( $\mathrm{m}, \mathrm{n}, \mathrm{p}$ and $\mathrm{q}$ ) are calculated by using both finite differences and dual numbers automatic differentiation. The base values of the parameters at which the sensitivities are calculated are 2.0, 7.0, 2.0 and 2.0 for $\mathrm{m}, \mathrm{n}, \mathrm{p}$ and $\mathrm{q}$, respectively. For finite difference derivatives, $1 \%$ of base values of the parameters are used. The sensitivities of the model outputs to its parameters are calculated as follows,

$$
S_{\phi, V}=\frac{\Delta V}{\max (\phi)} \frac{d \phi}{d V}
$$

Where $S_{\phi, V}$, is the sensitivity of $\phi$ with respect to $V$. In this study, $\phi$ represents electrical potential, activation loss, mass transfer loss and ohmic loss. Parameters, m, n, p, and q are represented by $V$. Cell potential drop, polarization losses and ohmic loss (Figure $4.6 \mathrm{a} \& \mathrm{~b}$ ) at gradual degradation times are predicted under phosphine exposure by using the parameters that are listed on Table 4.1. The model parameters are calibrated using the experiments performed by Guo et al. [19]. In this 
case activation loss is much larger than the mass transfer and ohmic loss. The magnitude of ohmic loss is too low to affect the cell performance. Therefore the performance loss is virtually due to the activation over potential and mass transfer losses as it is depicted in Figure $4.6 \mathrm{~b}$. 


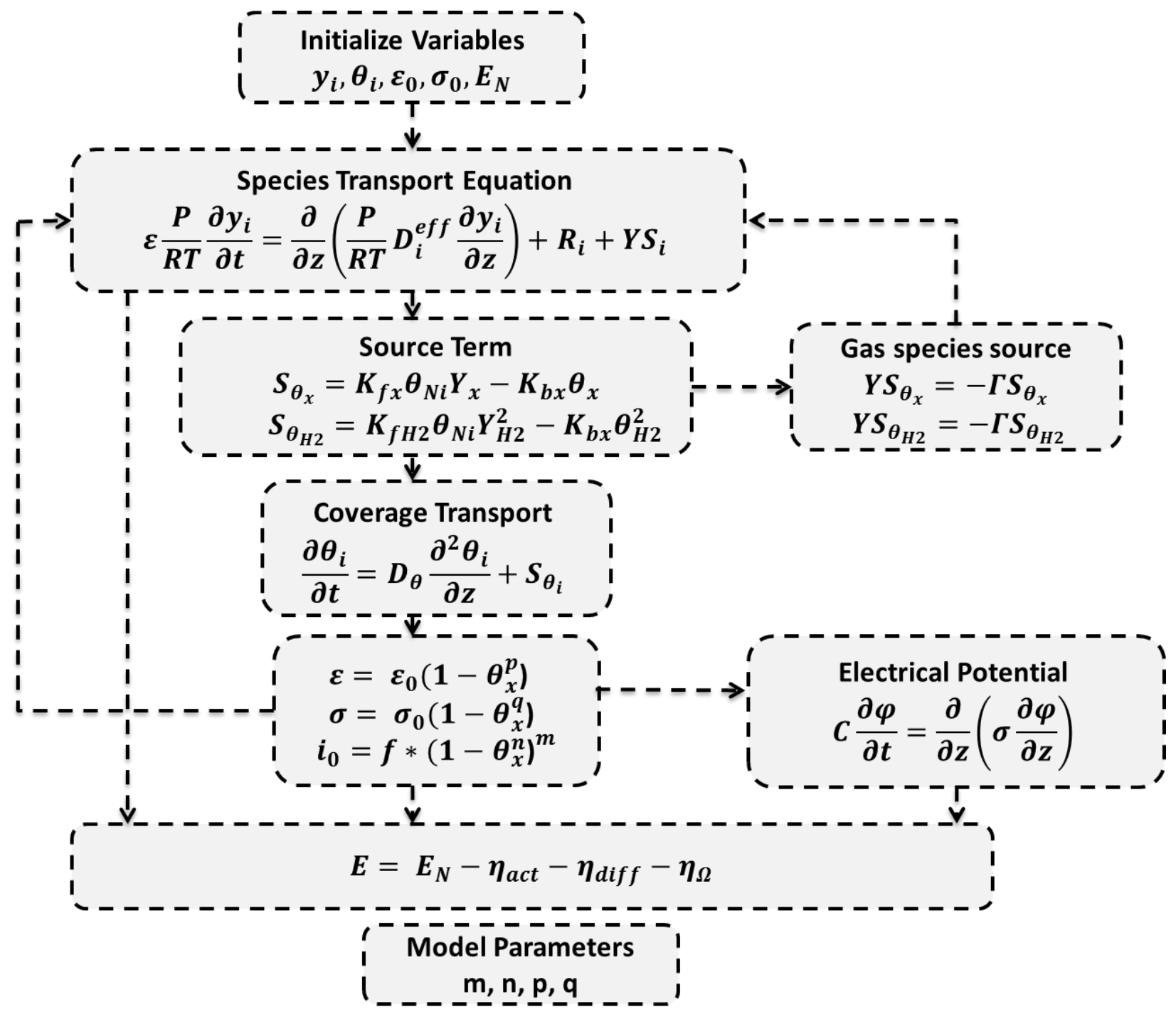

Figure 4.5: Flow chart of transient one-dimensional performance degradation model and its transport equations. 
Following the same procedure the DNAD and FD derivatives of the electrical potential, mass, activation and ohmic losses with respect to the model parameters $\mathrm{m}, \mathrm{n}, \mathrm{p}$, and $\mathrm{q}$ are obtained (Figure 4.7-10). As it is shown on model flow chart (Figure 4.5), $\mathrm{m}$ and $\mathrm{n}$ parameters are just related to the anode exchange current density. Therefore, the derivatives with respect to these parameters influence cell electrical potential and activation losses. As it can be seen in Figure 4.7, derivative of electrical potential with respect to parameter $m$ increased during gradual degradation, whereas the derivative of activation losses decreased. However, an opposite behavior is observed in Figure 4.8 for derivatives of electrical potential and activation losses with respect to parameter $\mathrm{n}$. This means that parameter $\mathrm{m}$ slows the degradation process, on the other hand, parameter $\mathrm{n}$ accelerates the degradation process. In addition, during the transient degradation of anode, the effect of parameters $\mathrm{n}$ and $\mathrm{m}$ increases. Therefore, it can be concluded that the cell rapid performance loss is very close to the cell failure time and it is due to the accelerated activation losses which are controlled by the parameter $\mathrm{m}$ and $\mathrm{n}$.

In Figure 4.9, derivatives with respect to parameter $\mathrm{p}$ are given. It is seen that electrical potential and all losses are influenced (Figure 4.5) by the parameter p, which is an exponent in the porosity equation. Actually this behavior is expected and it can be seen from the flow chart of the model. The change in porosity affects the pore volume fraction inside the anode. Therefore, the diffusion coefficients of each gaseous species are under the influence of parameter $\mathrm{p}$. Increasing parameter $\mathrm{p}$ decreases the effect of the coverage $(0<\theta<1)$ on the pore volume. Consequently, mass transfer loss decreases by increasing parameter $\mathrm{p}$. In addition, the diffusion coefficient of the phosphine is a function of the pore volume, therefore increasing parameter $\mathrm{p}$, which is indeed decreasing the influence of the coverage on pore volume, will have a positive contribution to the phosphine propagation. As a result of this fact, the coverage will reach the active layer faster. 
Namely, an increase in parameter $\mathrm{p}$ contributes a growing effect on ohmic and activation losses, hence electrical potential decreases. The influence of the parameter $\mathrm{q}$ is shown in Figure 4.10. Where it is seen that the effect of the parameter q on electrical potential is quite low compared with the other parameters; this is due to the effect of the ohmic loss on electrical potential. Even though, the ohmic loss is sensitive to the parameter $\mathrm{q}$, it does not affect the overall cell performance, because the ohmic losses inside the SOFC are mostly due to the ionic conductivity of the electrolyte. However, the phosphine exposure does not affect the ionic conductivity inside the electrolyte. Therefore in the 1D performance degradation model, the effect of the ohmic loss could be neglected. The magnitudes of the sensitivity of the model outputs, electrical potential and losses, with respect to listed parameters usually increased during the gradual degradation time. As it can be seen in Figure 4.6, the cell potential drop is sharp towards the cell failure time. At this sudden decrease in cell performance, the model is very sensitive to its parameters. As a result, one can say that the sensitivity analysis provide a better understanding of the model and its parameters effects. 

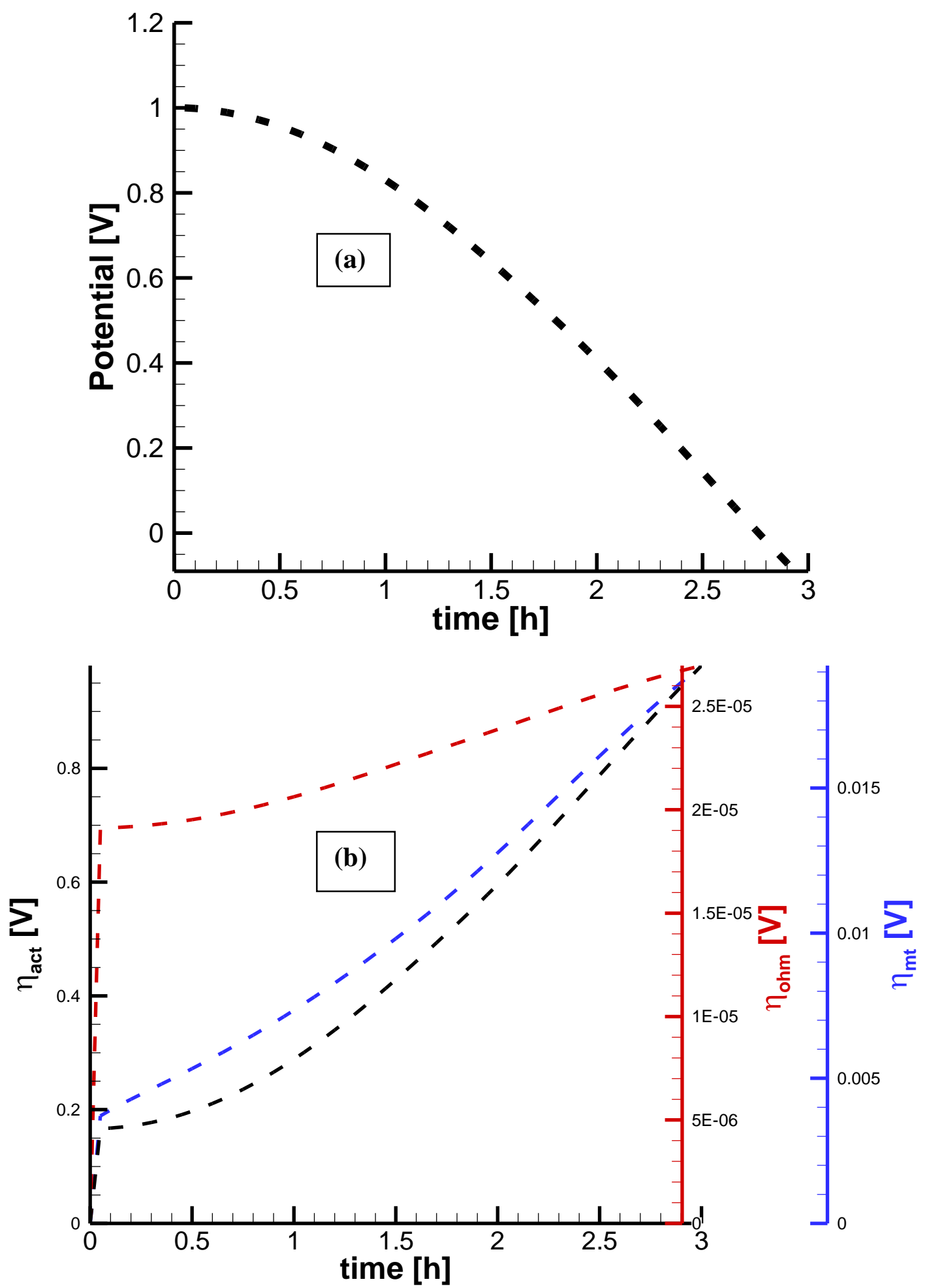

Figure 4.6: Performance degradation due to $10 \mathrm{ppm} \mathrm{PH}_{3}$ at $0.1 \mathrm{~A} / \mathrm{cm}^{2}$ current density. (b) Losses during gradual degradation for wet fuel case predicted by the degradation model. 

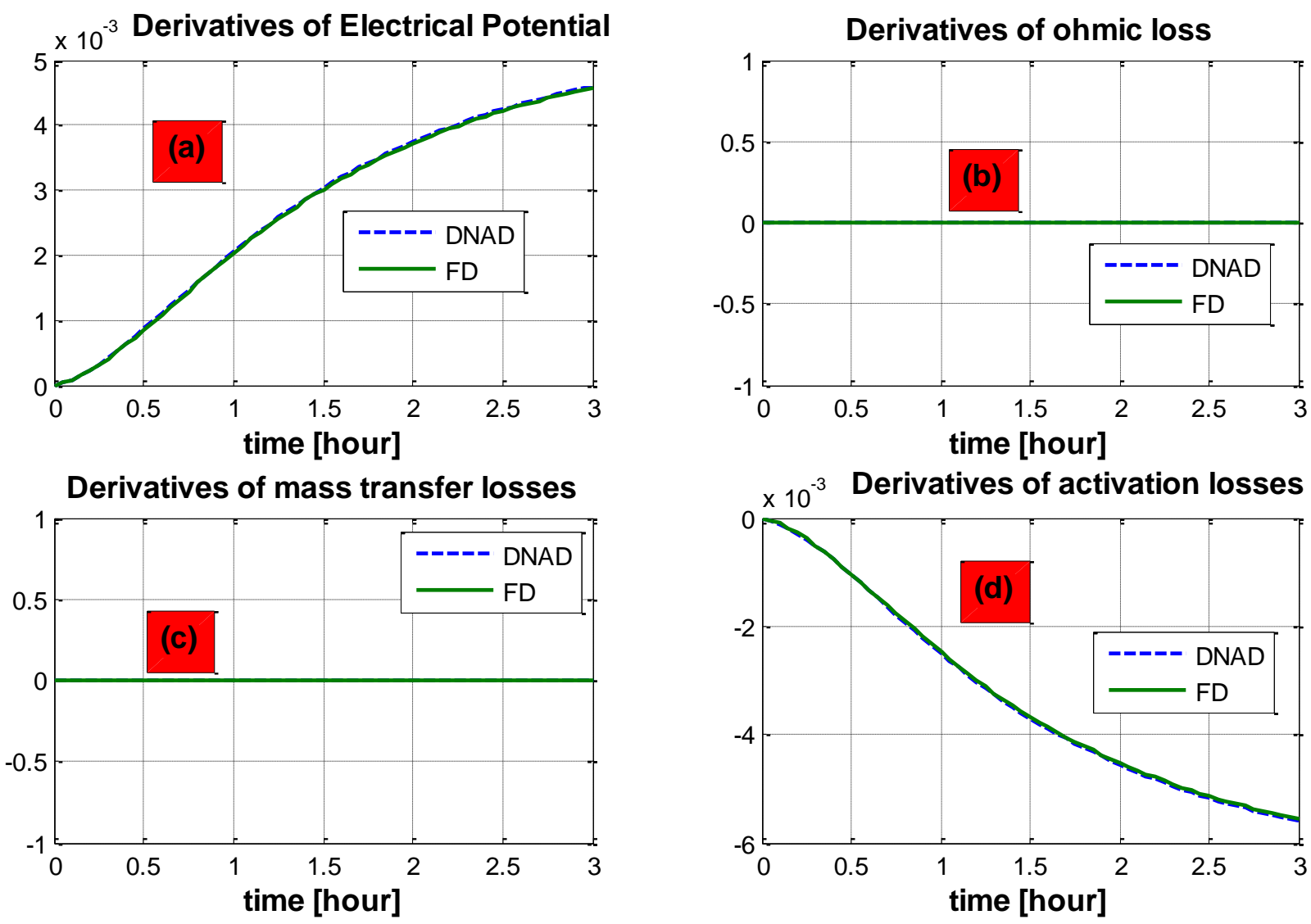

Figure 4.7: Dimensionless derivative of (a) electrical potential, (b) ohmic losses, (c) mass transfer losses (d) activation losses with respect to parameter $\mathrm{m}$ 


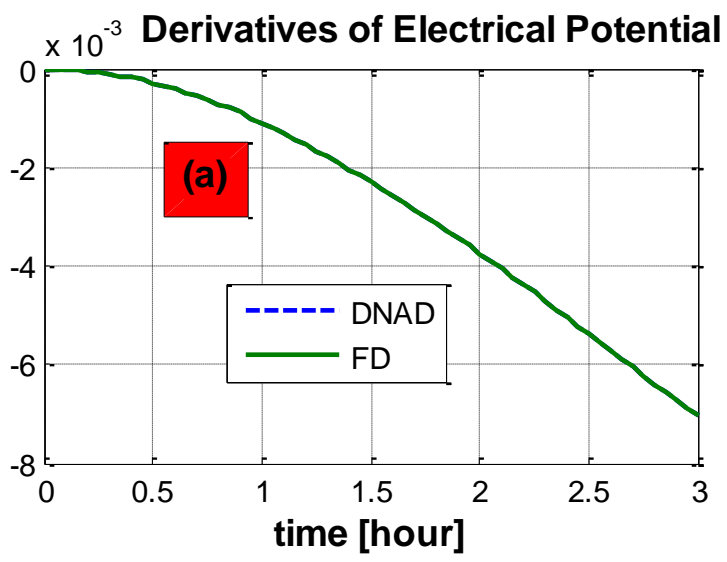

Derivatives of mass transfer losses

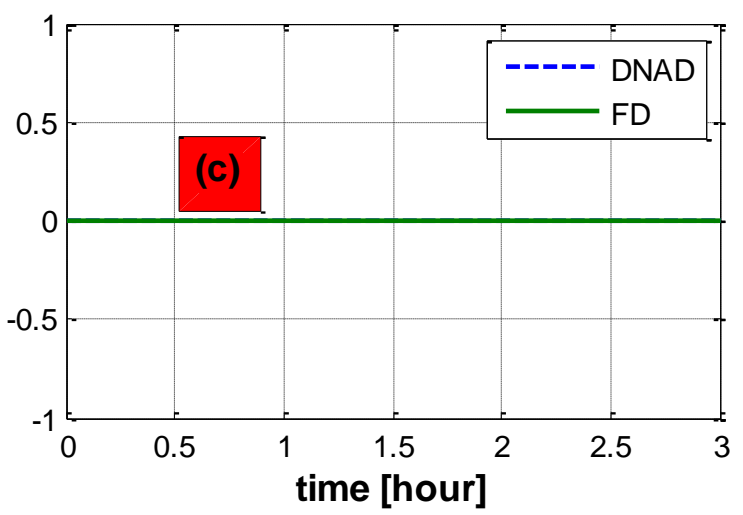

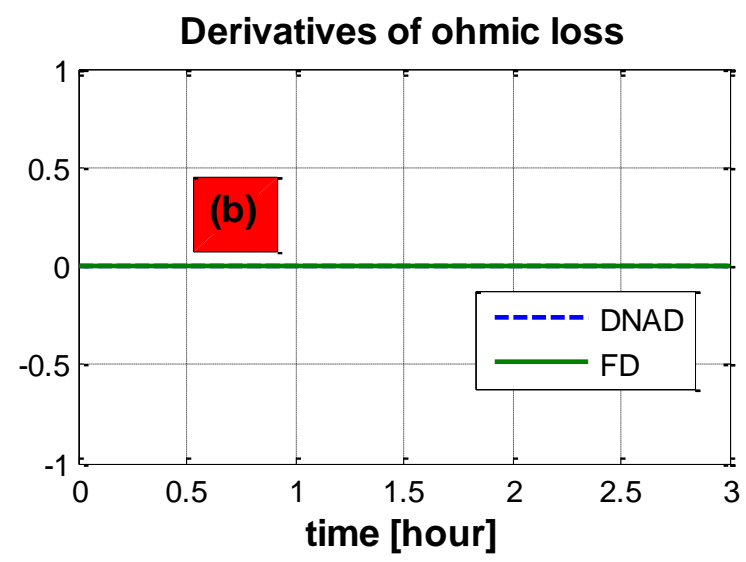

Derivatives of activation losses

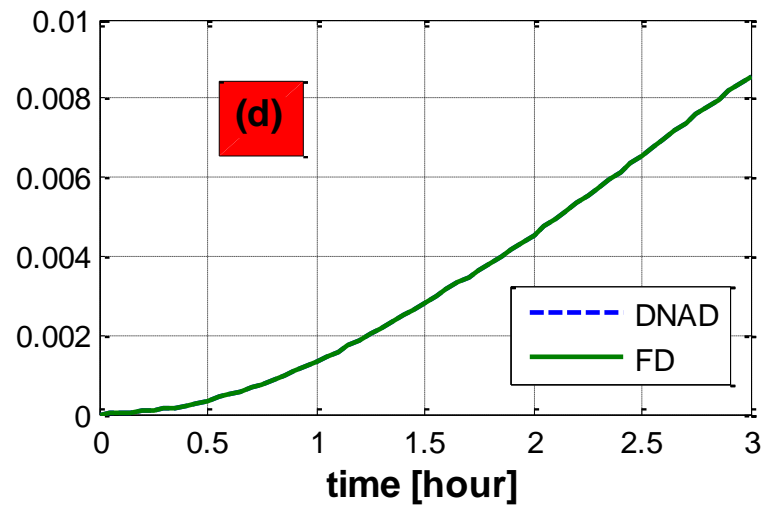

Figure 4.8: Dimensionless derivative of (a) electrical potential, (b) ohmic losses, (c) mass transfer losses (d) activation losses with respect to parameter $\mathrm{n}$ 

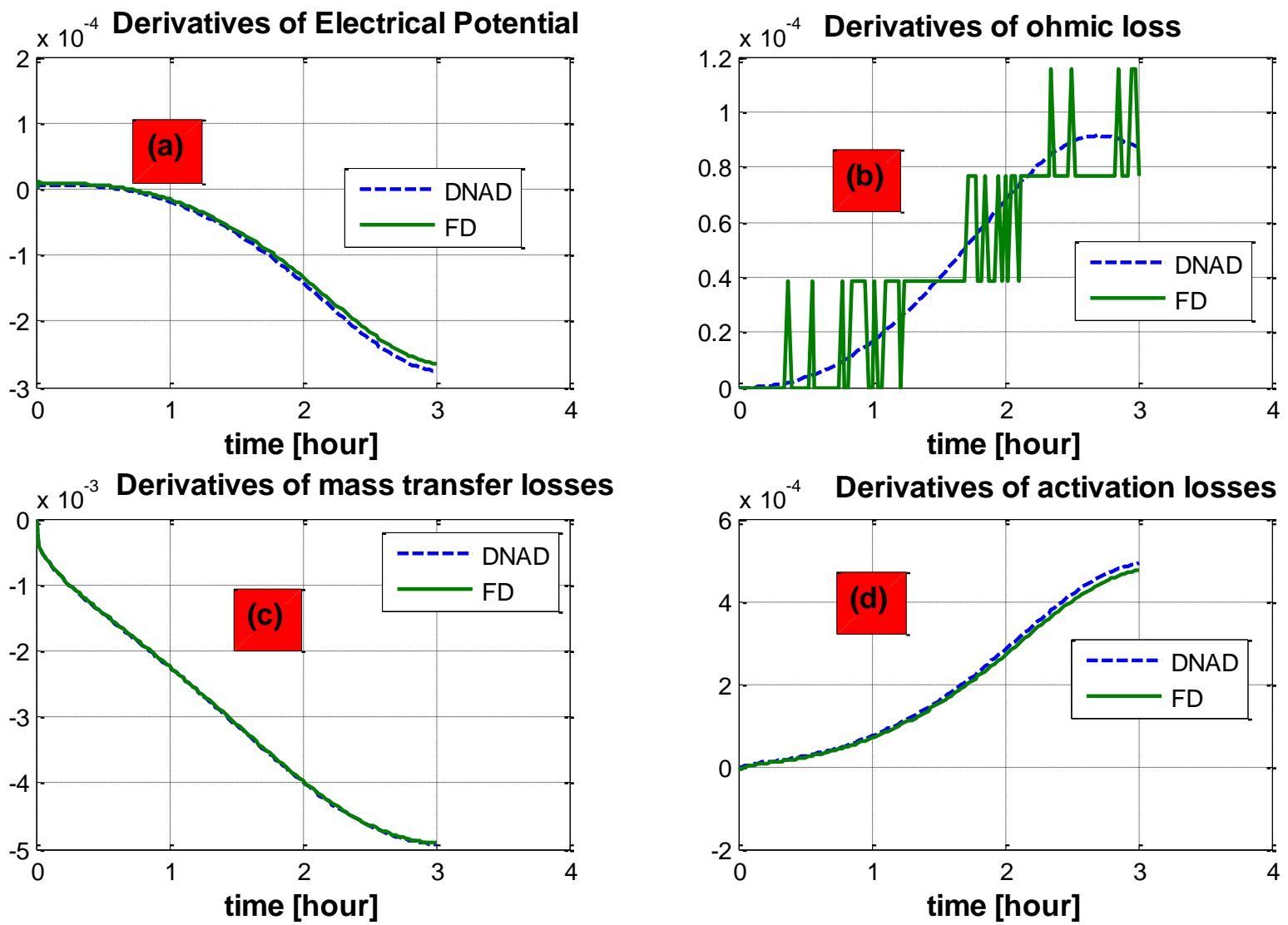

Figure 4.9: Dimensionless derivative of (a) electrical potential, (b) ohmic losses, (c) mass transfer losses (d) activation losses with respect to parameter $\mathrm{p}$ 

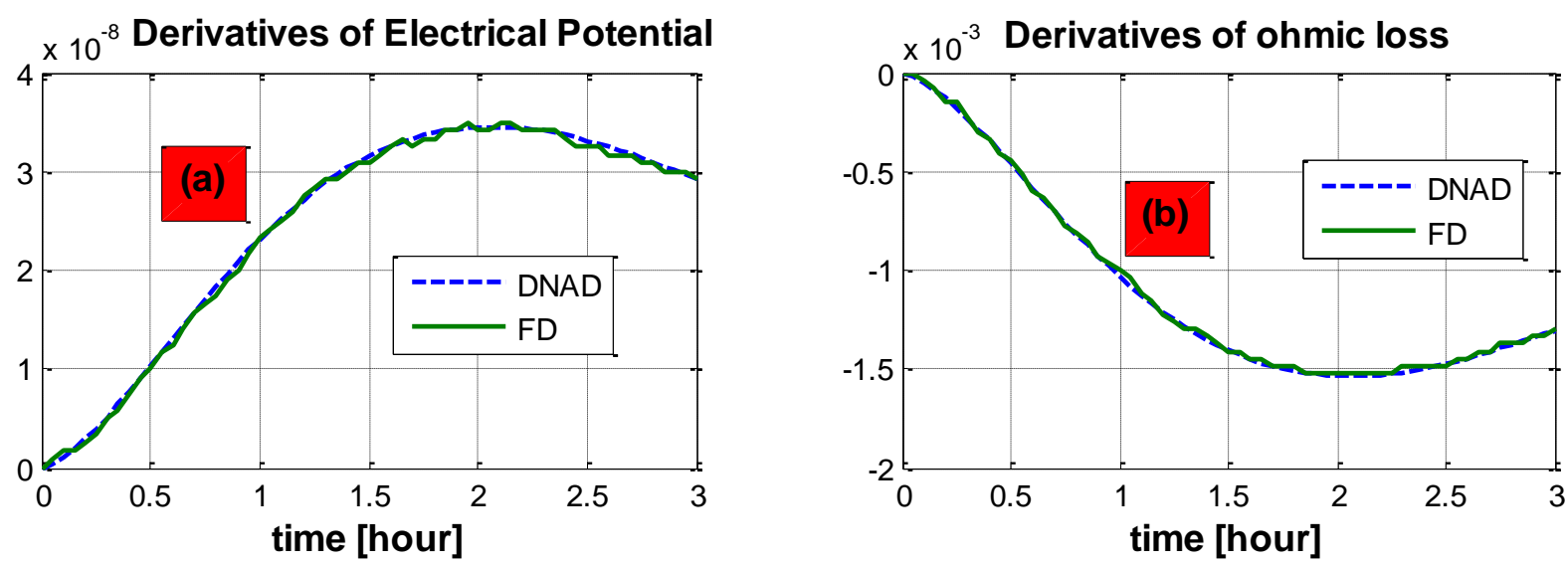

Derivatives of mass transfer losses

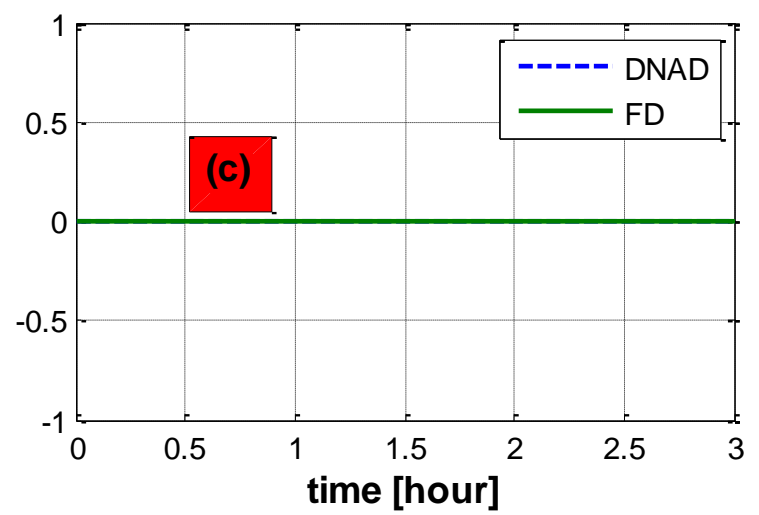

Derivatives of activation losses

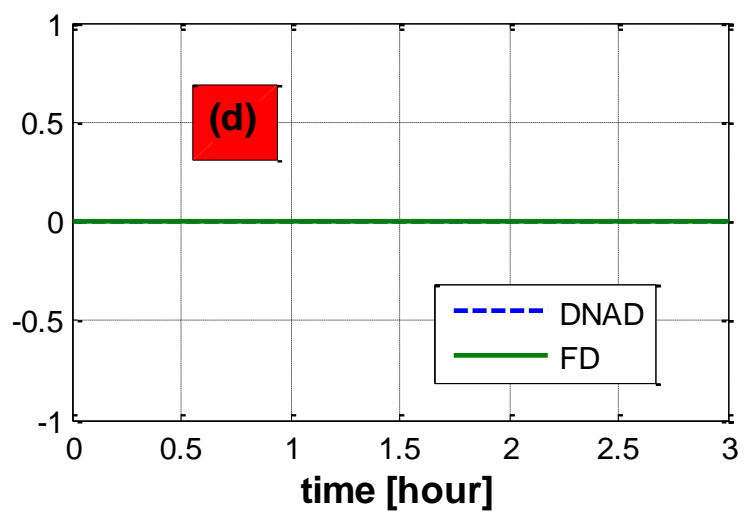

Figure 4.10: Dimensionless derivative of (a) electrical potential, (b) ohmic losses, (c) mass transfer losses (d) activation losses with respect to parameter $\mathrm{q}$ 


\subsection{Conclusions}

- Dual Number Automatic Differentiation (DNAD) is an elegant approach to finding derivatives of numerical solutions to model parameters, including material properties.

- The sensitivities obtained from DNAD could be different from the exact derivatives of the unknown true solution to the mathematical model due to inherent errors in numerical solution, in particular the discretization error.

- DNAD produces more accurate and smooth derivatives compared to the FD approach.

- It is possible to calculate derivatives with respect to more than one independent variable simultaneously by adding more components to the dependent variables with DNAD method.

The application of DNAD to calculate the sensitivity of a one-dimensional SOFC Degradation Model, to the empirical parameters has shown the following;

- The DNAD and FD results agree with each other closely

- Sensitivity to most parameters increases as the cell degradation increases.

- In transient calculations model sensitivity analyses should be performed as the state of the cell changes. In this regard, DNAD approach would be very advantageous. 


\section{CHAPTER 5 - Extension of the degradation model to three-dimensional planar cell}

Part of this work was published in ECS Trans 57(1): 2679-2689, 2013 [62]

\subsection{Introduction}

A three dimensional code developed in house (DREAM SOFC) which is capable of simulating the performance of SOFCs operating with a variety of fuel mixture was enhanced to simulate the fuel contaminant performance degradation of anode supported planar cell. A brief description of the code follows:

DREAM SOFC's calculations are based on a cell level model which includes current collectors, anode, electrolyte, cathode and gas and fuel channels. Temperature distribution is solved for each component of the cell. The mass transport of gas species is solved along the gas channels, and inside the porous anode and cathode. The specie coverage on active nickel sites is solved with the porous anode similar to the one dimensional degradation model. The charge transport equations are solved for the entire cell. Electrochemical reactions are solved for both $\mathrm{CO}$ and $\mathrm{H}_{2}$. The code is capable of simulating a mixture of $\mathrm{CO}$ and $\mathrm{H}_{2}, \mathrm{H}_{2} \mathrm{O}$ and $\mathrm{N}_{2}$ on the fuel side and for $\mathrm{O}_{2}$ and $\mathrm{N}_{2}$ on the cathode side. In this study, phosphine induced performance degradation of a planar cell will be investigated. The empirical parameters were first calibrated using the one dimensional model 
and then a sensitivity analysis of the model was performed to understand the behavior of the model with respect to its parameters. Eventually, the information that was revealed from the one dimensional model was used to predict fuel contaminant performance degradation of a planar cell. The details are discussed in subsequent sections.

The detailed information for the mathematical model used in DREAM SOFC can be found in $[47,48,53]$. DREAM SOFC is extended to enable calculation of phosphine induced performance degradation of a three-dimensional planar SOFC. As shown in Figure 5.1, porosity, exchange current density, electrical conductivity and transport of gaseous species inside the anode are functions of local contaminant coverage. These parameters are calculated by implementing the phenomenological performance degradation model [6,12] into current three-dimensional model. The electrochemical reactions are assumed to occur only at anode/electrolyte interface and hence the exchange current is a function of contaminant coverage in this region. It should be noted here that the transport of contaminant coverage also affects the transport properties inside the anode. The original, one-dimensional model $[6,12]$, was successfully applied to predict performance degradation of button cells. Incorporation of the same model into a 3D SOFC code made it possible to predict performance degradation in planar SOFC due to fuel contaminants. Unsteady threedimensional performance degradation of planar DREAM-SOFC model takes a very long computational time compared to one-dimensional degradation model in button cells. Thus, the empirical model parameters were calibrated with one dimensional model using data from button cell experiments. The calibrated parameters from the one dimensional model are then used to perform simulations to predict fuel contaminant performance degradation of a planar cell. 


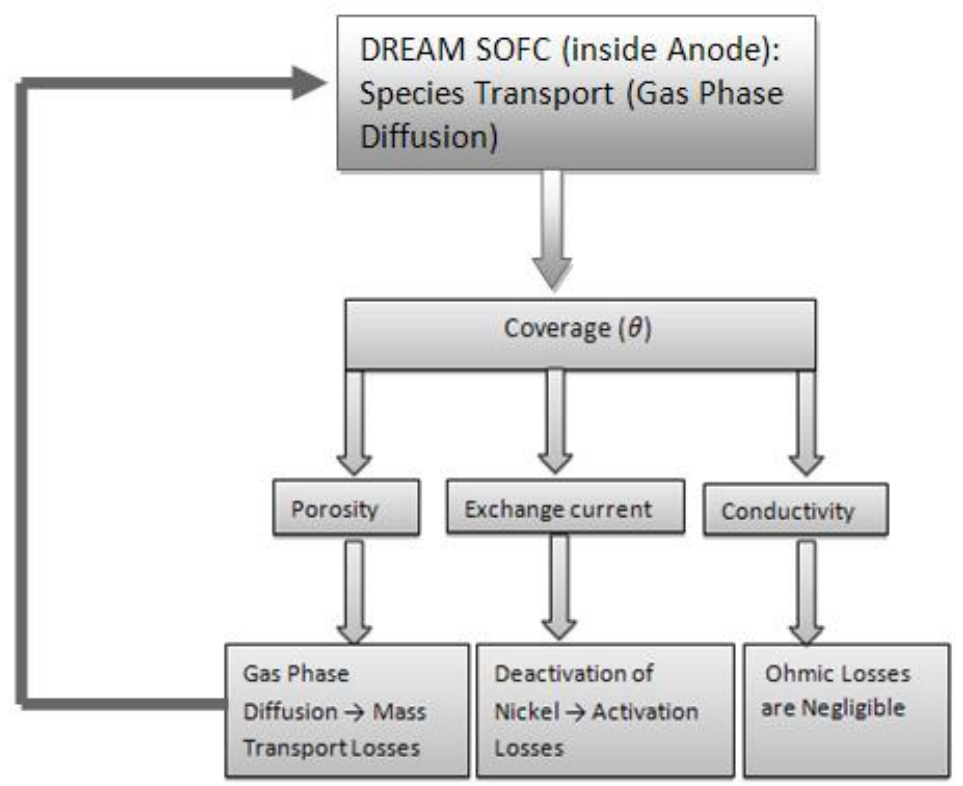

Figure 5.1: DREAM SOFC integration with degradation model

The degradation model employed in this work considers the competitive adsorption of hydrogen and the phosphine on the nickel catalyst surface inside the anode. The monolayer adsorption mechanism $[6,12]$ for the formation of secondary phases and hydrogen adsorption is described by

$$
\begin{aligned}
& P H_{3}+N i \stackrel{\stackrel{k_{f, P H_{3}}}{\longrightarrow}}{\stackrel{k_{b, P H_{3}}}{\longrightarrow}} N i-P H_{3} \\
& H_{2}+2 N i \stackrel{k_{f, H_{2}}}{\stackrel{k_{b, H_{2}}}{\longrightarrow}} 2(N i-H)
\end{aligned}
$$

In eqs. (5.1 and 5.2) $k_{f, \mathrm{PH}_{3}}, k_{b, P H_{3}}$ and $k_{f, H}, k_{b, H}$ are forward and backward reactions rates for formation of secondary phases and adsorption of hydrogen respectively. A three-dimensional 
phenomenological transport equation, $[6,12]$ for the coverage of phosphine propagation inside the anode is given as:

$$
\frac{\partial \theta_{i}}{\partial t}=D_{\theta} \nabla^{2} \theta_{i}+\omega_{\theta_{i}}
$$

where $\theta_{i}$ is the surface coverage of phosphine. $D_{\theta}$ is the transport coefficient for the coverage which is taken as $1 \times 10^{-19} \mathrm{~m}^{2} / \mathrm{s}[6,12] . \omega_{\theta_{i}}$ is the source term for formation of secondary phases. Detailed information on the performance degradation model can be found in Cayan et al. [12]. The functional dependency of porosity and exchange current density of hydrogen to local contaminant coverage is given as follows, [46,12];

$$
\begin{aligned}
& \varepsilon=\varepsilon_{0}\left(1-0.95 \theta^{p}\right) \\
& i_{o, H_{2}}=c_{1}\left(\frac{y_{H_{2}, \text { int }}}{y_{H_{2}, \text { ref }}}\right) \exp \left(-\frac{E_{a c t, H_{2}}}{R_{u} T}\right)\left(1-\theta_{N i-P H_{3}, \text { int }}^{m}\right)^{n}
\end{aligned}
$$

Where $\varepsilon_{0}, \varepsilon$, and $\theta$ are initial porosity, porosity, and phosphine coverage, respectively. In eq. (5.5), $\mathrm{m}$ and $\mathrm{n}$ are the model parameters obtained from the available experimental data for button cell. The constant $C_{1}$ is $3.936 \times 10^{7}$, the hydrogen reference concentration, $y_{H_{2}, r e f}$, is 0.333 , the hydrogen activation barrier, $E_{\mathrm{H}_{2}}$,act is $91.661 \mathrm{~kJ} / \mathrm{mol}$ [6], and $\mathrm{Ni}-\mathrm{PH}_{3}$, int is the phosphine coverage at the anode/electrolyte active interface. 


\subsection{Validation of the degradation model using PNNL experiments}

\subsubsection{Simulation approach}

SOFC anode performance degradation caused by fuel contaminants is altered by operating conditions (temperature, current load and fuel utilization) and fuel composition (contaminant concentrations). In this section, an attempt is made to simulate anode contaminant test that was performed in Pacific Northwest National Laboratory (PNNL) by Stevenson et al. [63]. The numerical simulation scheme presented in Figure 5.1 is used to predict experimentally observed elemental redistribution of $\mathrm{SOFC}$ anode exposed to $1 \mathrm{ppm} \mathrm{AsH}_{3}$ in $50 \mathrm{~h}$. Figure 5.2 represents the experimental setup used by Stevenson et al. Operating conditions, fuel concentrations, cell geometry parameters and calibrated degradation model parameters are listed in Table 5.1. The fuel utilization that is given on Table 5.1 corresponds to $0.8 \mathrm{~A} / \mathrm{cm}^{2}$ current density. But the experiment and simulations are performed at open circuit voltage (OCV). The cell is modeled in 3D along $5.08 \mathrm{~cm}$ long fuel channel and through a 50/50 \% vol Ni/YSZ 500 microns thick anode with $40 \%$ porosity. Gas phase transport along the fuel channel takes place via diffusion and convection. However, transports of gaseous species in the pore space in the solid phases occur via diffusion only and diffusion is modeled by Knudsen and binary diffusion mechanisms. Interested readers on the details of the diffusion model are referred to Cayan [6]. Simulations were performed with two different mass transfer coefficients of contaminant from fuel channel to porous anode. The

degradation model parameters for $1 \mathrm{ppm} \mathrm{AsH}_{3}$ in utilized fuel containing $\% 55 \mathrm{H}_{2}$ and $45 \% \mathrm{H}_{2} \mathrm{O}$ were taken from Cayan [6]. 
Table 5.1: Model parameters used for $\mathrm{AsH}_{3}$ degradation and test conditions for the PNNL experiments

\begin{tabular}{|c|c|}
\hline \multicolumn{2}{|c|}{ Geometry Parameters of the planar SOFC } \\
\hline Anode thickness & $500 \mu \mathrm{m}$ \\
\hline Electrolyte thickness & $20 \mu \mathrm{m}$ \\
\hline Cathode thickness & $10 \mu \mathrm{m}$ \\
\hline Fuel channel height & $2.5 \mathrm{~mm}$ \\
\hline Air Channel height & $2.5 \mathrm{~mm}$ \\
\hline Cell length & $5.08 \mathrm{~cm}$ \\
\hline Current collector width & $1.27 \mathrm{~cm}$ \\
\hline Channel width & $2.54 \mathrm{~cm}$ \\
\hline \multicolumn{2}{|c|}{ Conditions and parameters for simulation } \\
\hline $\begin{array}{l}\text { Fuel inlet composition } \\
\text { (Mole \%) }\end{array}$ & $55 \% \mathrm{H}_{2}+45 \% \mathrm{CO}_{2}+1 \mathrm{ppm} \mathrm{ASH}$ \\
\hline Fuel inlet temperature $(\mathrm{K})$ & 1073 \\
\hline $\begin{array}{l}\text { Air inlet Composition (Mole } \\
\text { fractions) }\end{array}$ & $21 \% \mathrm{O} 2$ \\
\hline Air inlet temperature $(\mathrm{K})$ & 1073 \\
\hline Pressure (atm) & 1.01325 \\
\hline External Boundaries & Adiabatic \\
\hline Current density $(\mathrm{A} / \mathrm{cm} 2)$ & 0.0 \\
\hline Fuel Utilization $(\%)$ & 65 \\
\hline Air Utilization (\%) & 1.25 \\
\hline Fuel mass flow rate $(\mathrm{kg} / \mathrm{sec})$ & $4.9967 \mathrm{e}-006$ \\
\hline Air mass flow rate $(\mathrm{kg} / \mathrm{sec})$ & $4.4180 \mathrm{e}-005$ \\
\hline Anode porosity & 0.480 \\
\hline Cathode porosity & 0.45 \\
\hline Mass transfer coefficient & Case 1: $1 \mathrm{e}-5$ \\
\hline \multicolumn{2}{|c|}{ Degradation Parameters } \\
\hline $\boldsymbol{k}_{\boldsymbol{f}, \boldsymbol{x}}, \mathrm{s}^{-1}$ & $5.0 \times 10^{3} \exp (-5.97 / \mathrm{RT})$ \\
\hline $\boldsymbol{k}_{\boldsymbol{b}, \boldsymbol{x}}, \mathrm{s}^{-1}$ & $9.1 \times 10^{-1} \exp \left(-9.27 \times 10^{4} / \mathrm{RT}\right)$ \\
\hline $\mathrm{m}$ & 0.3 \\
\hline $\mathrm{n}$ & 6.5 \\
\hline $\mathrm{p}$ & 2 \\
\hline$q$ & 2.0 \\
\hline
\end{tabular}




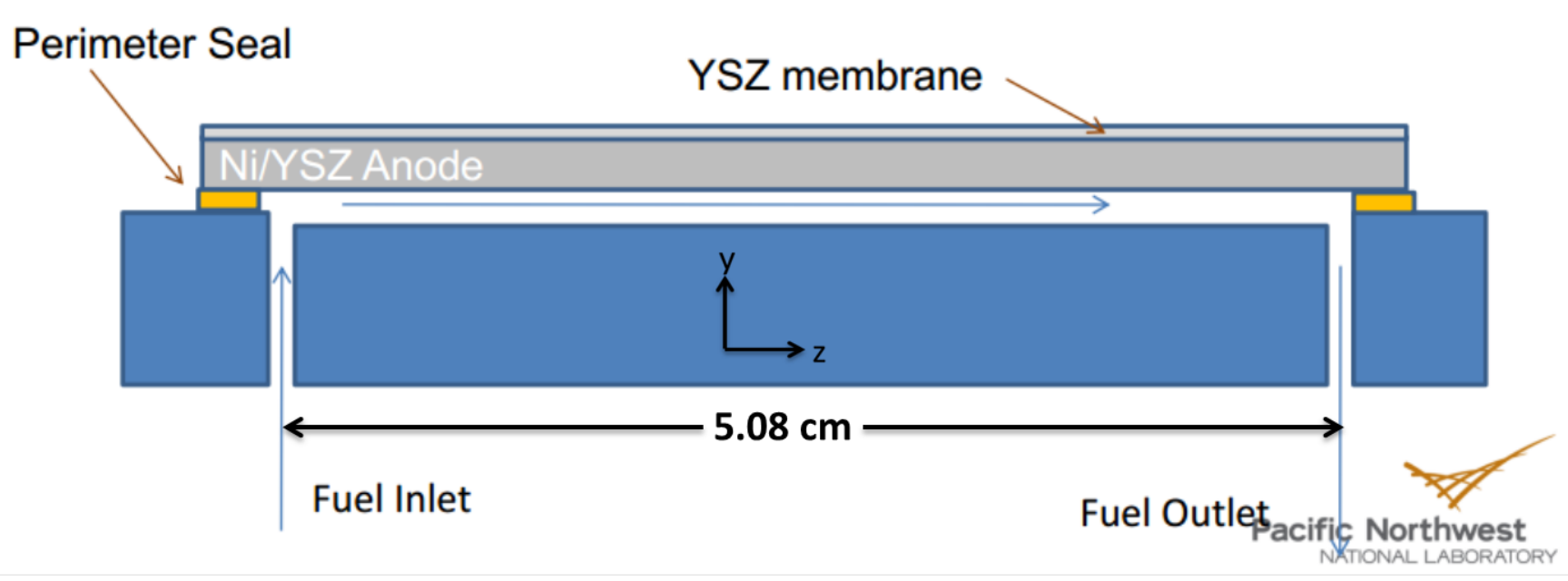

Figure 5.2: Geometrical representation of Channel and SOFC's anode by Stevenson et al. [63]

\subsubsection{Results and discussions}

Energy dispersion spectroscopy (EDS) area atomic concentrations profiles after $50 \mathrm{~h}$ of contaminant exposure are shown Figure 5.3 [63]. As it is depicted in EDS concentrations profiles the arsenide interaction with nickel is more effective near the surface and sharp boundaries occur between secondary phase formation regions and regions in which arsenide has not yet reacted with nickel yet. The results shown in Figure 5.4 and Figure 5.5 are obtained with the simulation performed by using mass transfer coefficient of arsine from fuel channel to porous anode, $h_{\text {mass }}=$ $1 \times 10^{-5} \mathrm{~m} / \mathrm{s}$.

In Figure 5.4, gaseous normalized arsenide mole fraction profiles inside the anode are depicted with contour plots. As it is elucidated from the arsenide profiles, a similar sharp boundary is created between the region near the surface and region far from the surface. 
The contaminant coverage in this model is designed as a function of local contaminant mole fraction and available nickel active sites. This functional dependency at different times of anode exposed arsine is depicted in Figure 5.5. Change in the trace element normalized coverage inside the anode is slightly smoother than that of the trace element mole fraction.

This smoothness can be explained by the functional dependency of coverage formation with the nickel active sites and trace element mole fraction. Upon arsine exposure, the nickel active sites on the surface layer are the first to react and form trace element coverage causing the nickel active sites to decrease further at the surface. This decelerates the rate of formation of the trace element coverage further near surface where the sharp boundary is located for arsine mole fraction. As it is seen from the simulated results, Figure 5.3 and Figure 5.5, the model is able to predict trends seen in the experimental data quite well.

In order to assess the predictions of the three-dimensional model (DREAM-SOFC) quantitatively, the normalized arsine atomic percentage obtained from EDS measurements is used. Here it should be noted that the arsine atomic percentage is the total arsine atoms, which are the compounds of the secondary phases only, but not the arsine gaseous phase. In this study, it is assumed that the secondary phases can be represented by the trace element coverage. Therefore, for the comparison between experiment and simulations, the normalized coverage profiles used are extracted from the simulations. As it is seen in Figure 5.6, the simulations by DREAM-SOFC show good agreement with the PNNL experiment. In both simulations and EDS analysis, the arsine nickel interaction near the surface is much stronger than the regions far from the surface. The simulation with lower arsine mass transfer coefficient has a better agreement with experiment than that of higher mass transfer coefficient. This parameter is difficult to determine either experimentally or theoretically, 
while the mass transfer from the channel to the porous electrode is altered significantly as secondary phases form and change the morphology of the electrode surface.

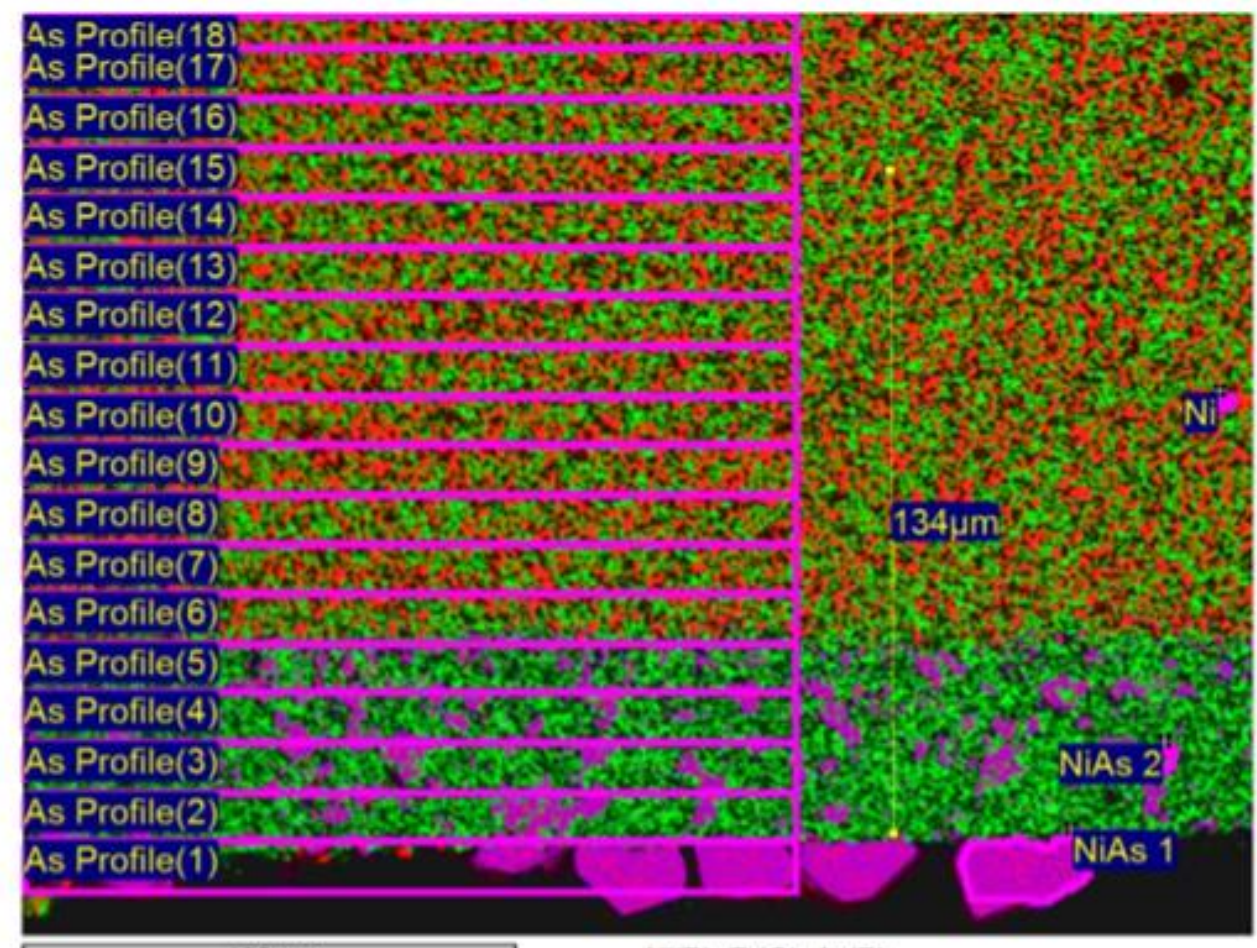

$100 \mu \mathrm{m}$

$\mathrm{Ni}(\mathrm{R}), \mathrm{Zr}(\mathrm{G}), \mathrm{As}(\mathrm{B})$

Profile height $=10 \mu \mathrm{m}$

Figure 5.3: (EDS) area atomic concentrations profiles after $50 \mathrm{~h}$ of $\mathrm{AsH}_{3}$ exposure by

Stevenson et al. [63]. 


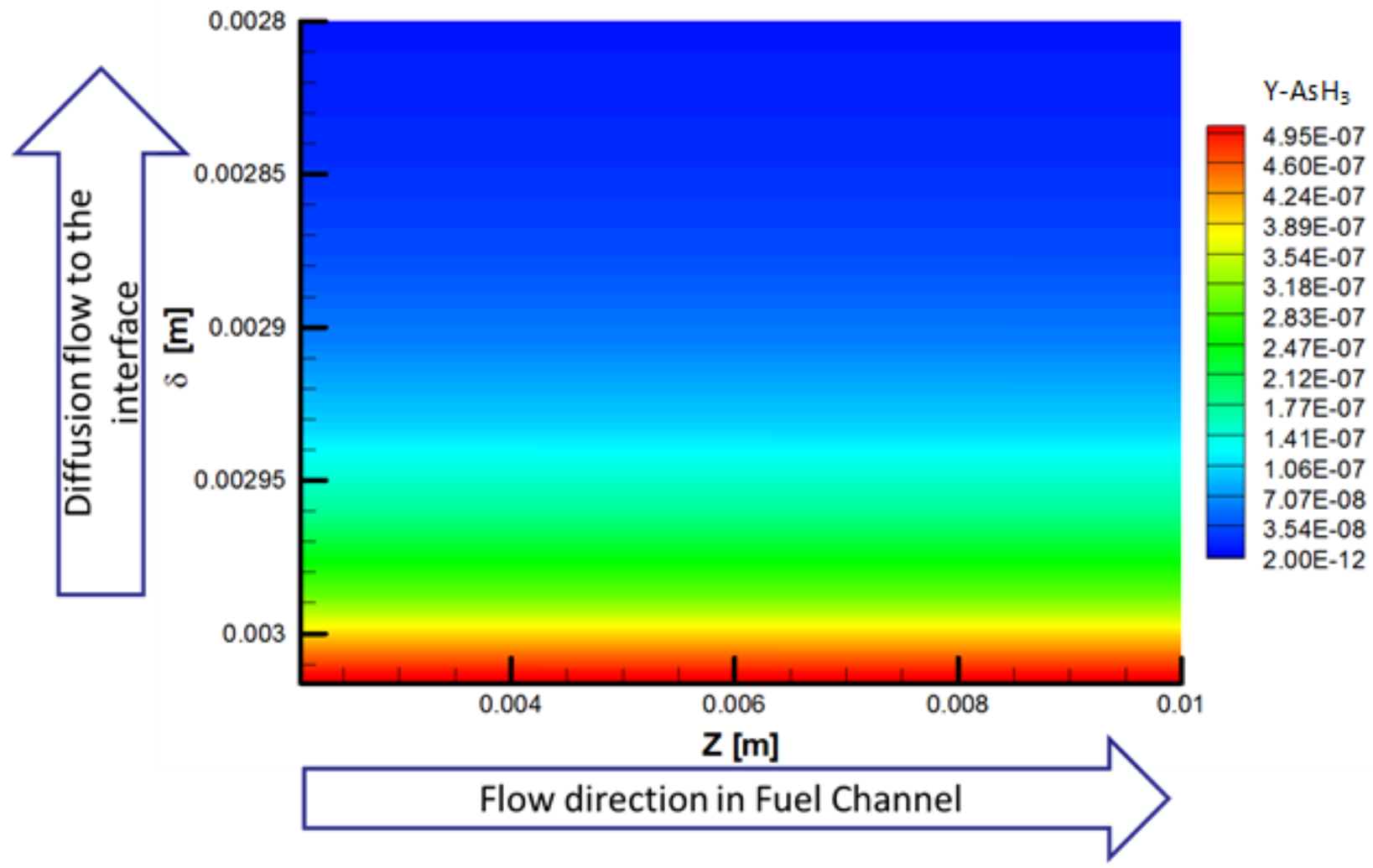

Figure 5.4: $\mathrm{AsH}_{3}$ concentration inside anode after $50 \mathrm{~h}$ of $1 \mathrm{ppm} \mathrm{AsH}_{3}$ 


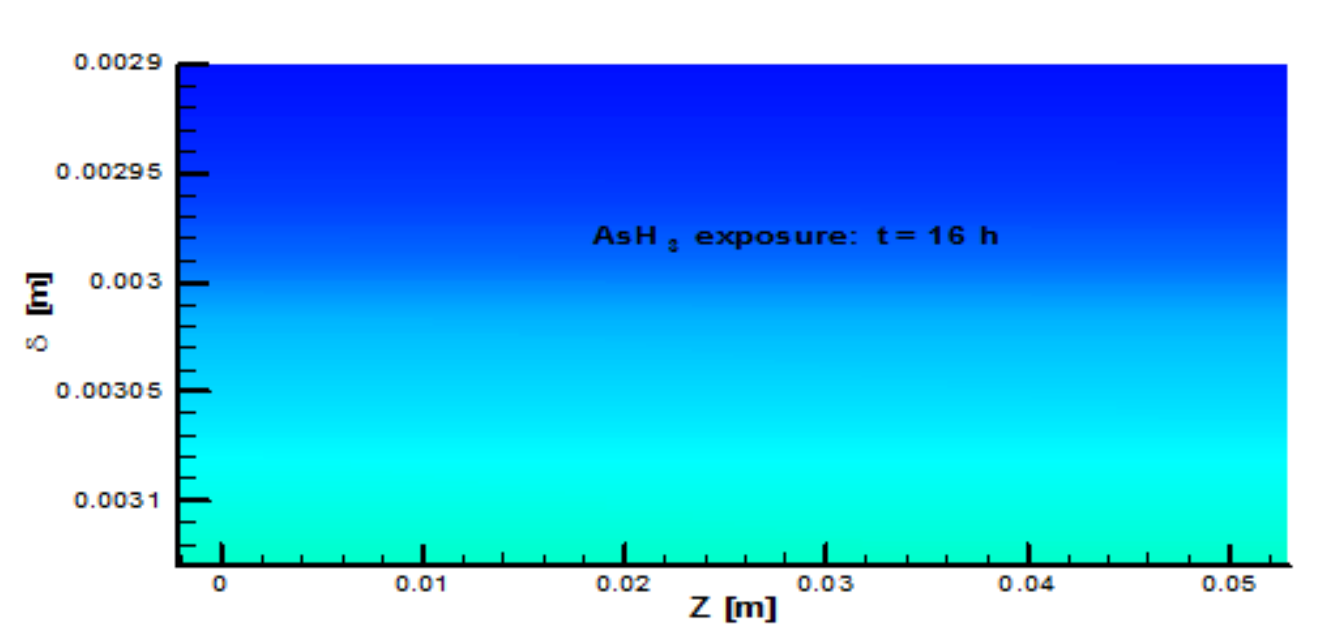

$\theta-\mathrm{AsH}_{3}$
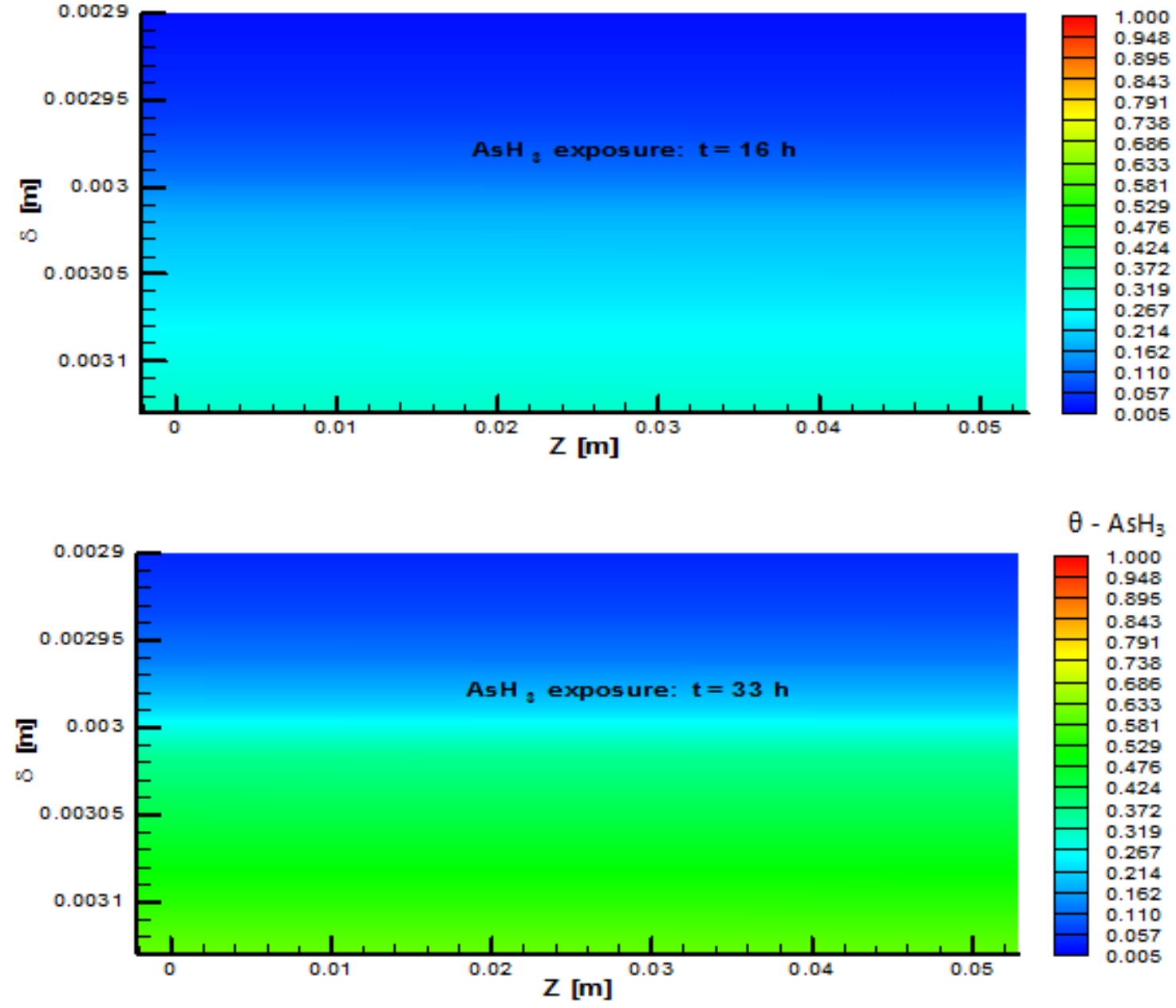

$\theta-\mathrm{AsH}_{3}$
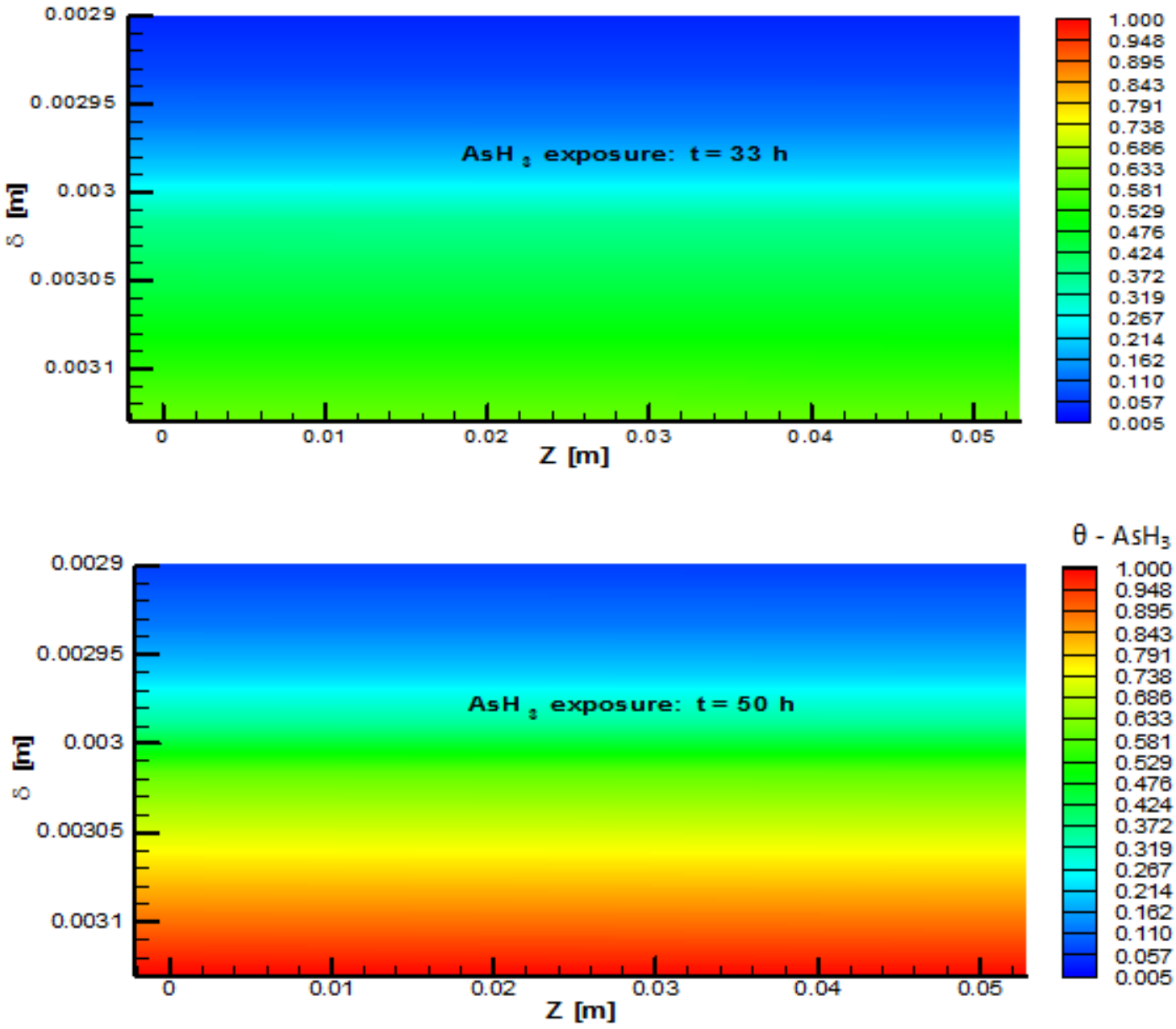

$\theta-\mathrm{AsH}_{3}$

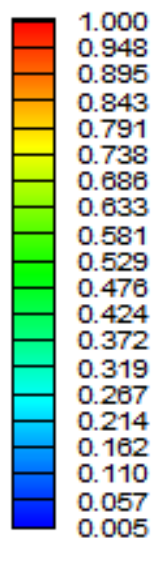

Figure 5.5: Normalized $\mathrm{AsH}_{3}$ coverage concentrations inside porous anode at 16, 33, and 50 $h$ of anode exposed to $\mathrm{AsH}_{3}$ 


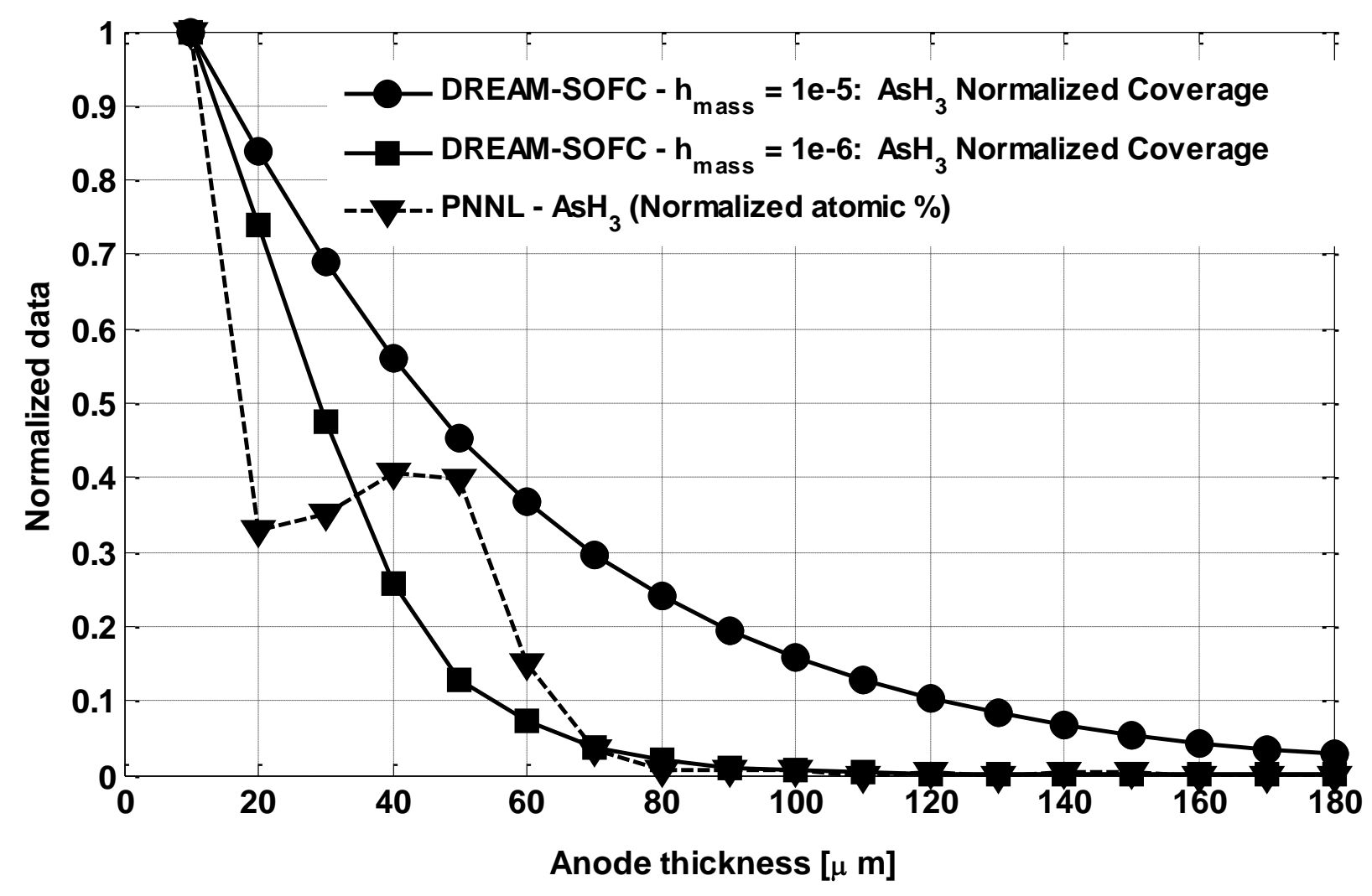

Figure 5.6: Normalized atomic concentrations profiles at $50 \mathrm{~h}$ of arsine exposure from EDS analyses by Stevenson et al. [63]. and normalized arsine coverage at $50 \mathrm{~h}$ of arsine exposure from simulations performed with two different arsine mass transfer coefficients $\left(h_{\text {mass }}=\right.$ $1 \times 10^{-5}$ and $1 \times 10^{-6}$ ) from fuel channel to porous anode

Here it should be noted that the transport of the arsine inside the fuel channel is solved by convection and diffusion, whereas inside the porous anode only diffusion is considered. At the boundary between fuel channel and porous anode the mass transfer coefficient is used to determine the mass flow of the arsine from the channel to the electrode. 


\subsubsection{Conclusions for simulations of PNNL $\mathrm{AsH}_{3}$ experiments}

Experimentally observed elemental redistribution of anode supported SOFC due to arsine exposure is predicted by using a multi-dimensional in-house code (DREAM-SOFC) designed for simulations of large planar SOFCs. Good agreement is achieved between EDS analysis and simulation results. Experimentally observed sharp boundaries for the secondary phases formation are obtained by simulations for the trace element coverage by $\mathrm{AsH}_{3}-\mathrm{Ni}$ interaction. Simulations and experimental analyses indicate that for fuels contain low contaminant concentrations $(\leq 1 \mathrm{ppm})$ trace element coverage can still occur, but the low rate of contaminant propagation can significantly reduce performance degradation rates of SOFCs anode. 


\subsection{Electrochemical evaluation of a planar SOFC under phosphine induced degradation}

\subsubsection{Introduction}

A multi-dimensional code (DREAM-SOFC) designed for simulation of large scale planar solid oxide fuel cells is extended by implementing a one-dimensional degradation model to predict the typical degradation patterns observed in SOFCs when the anode is exposed to phosphine. The empirical model parameters for the degradation part are calibrated using the accelerated button cell experiments as a guide. These parameters are then used in DREAM SOFC to predict phosphine induced performance degradation of the planar cell. For brevity, a planar cell with 10 channels is represented by only one channel cell which is exposed to phosphine so that the transient degradation due to phosphine exposure can be observed. In order to assess overall performance of the cell at different degradation levels, additional simulations are performed to predict the polarization behavior and the impedance response of the cell at the instantaneous of degradation levels. The objective of this chapter is to predict the experimentally observed patterns of phosphine induced degradation in planar cell SOFC utilizing hydrogen. The ultimate goal is to use the threedimensional transient code, DREAM-SOFC, to predict impedance and polarization response of the cell at different degradation levels. The following procedure is carried out to achieve this goal. A planar SOFC with 10 channels is used to calculate species, current and temperature distribution in the each component of cell until temperature and cell potential reach the steady state condition. Then with the same conditions a planar cell with one channel is chosen to represent the 10 channel cell. Further, the one channel planar cell is exposed to phosphine after the cell reached the steady state solution. The results are analyzed in the following sections. 


\subsection{Planar SOFC computational domain and operation conditions}

The computational domain used in the simulations is shown in Figure 5.7 and Figure 5.8 for 10 channel and one channel planar SOFC, respectively. The detailed geometric parameters and operation conditions used for the current simulations are given in Table 5.2. The only difference between 10 channels and one channel cell were the number of the channels and the width of the current collectors. One channel width is taken as $3 \mathrm{~mm}$ for both simulations whereas the current collectors width is $1.28 \mathrm{~mm}$ and $3 \mathrm{~mm}$ for one channel and 10 channel simulations, respectively.

Table 5.2: Geometry and operating conditions parameters for planar SOFC

\begin{tabular}{|l|r|}
\hline \multicolumn{2}{|c|}{ Geometry Parameters of the planar SOFC } \\
\hline Anode thickness & $50 \mu \mathrm{m}$ \\
\hline Electrolyte thickness & $170 \mu \mathrm{m}$ \\
\hline Cathode thickness & $50 \mu \mathrm{m}$ \\
\hline Fuel channel height & $2.5 \mathrm{~mm}$ \\
\hline Air Channel height & $2.5 \mathrm{~mm}$ \\
\hline Cell length & $5 \mathrm{~cm}$ \\
\hline Current collector width & $1.28 \mathrm{~mm}$ \\
\hline Channel width & $3 \mathrm{~mm}$ \\
\hline \multicolumn{2}{|c|}{ Conditions and parameters for simulation } \\
\hline \multicolumn{2}{|c|}{ (Mole \%) } \\
\hline Fuel inlet composition & $99.99 \% \mathrm{H} 2+10 \mathrm{ppm} \mathrm{PH3}$ \\
\hline \multicolumn{2}{|c|}{} \\
\hline Fuel inlet temperature (K) & 1073 \\
\hline Air inlet Composition (Mole fractions) & $21 \%$ O2 \\
\hline Air inlet temperature (K) & 1073 \\
\hline Pressure (atm) & 1.01325 \\
\hline External Boundaries & Adiabatic \\
\hline Current density (A/cm2) & 0.1 \\
\hline Fuel Utilization (\%) & 12.5 \\
\hline Air Utilization (\%) & 1.25 \\
\hline Anode porosity & 0.480 \\
\hline Cathode porosity & 0.45 \\
\hline
\end{tabular}




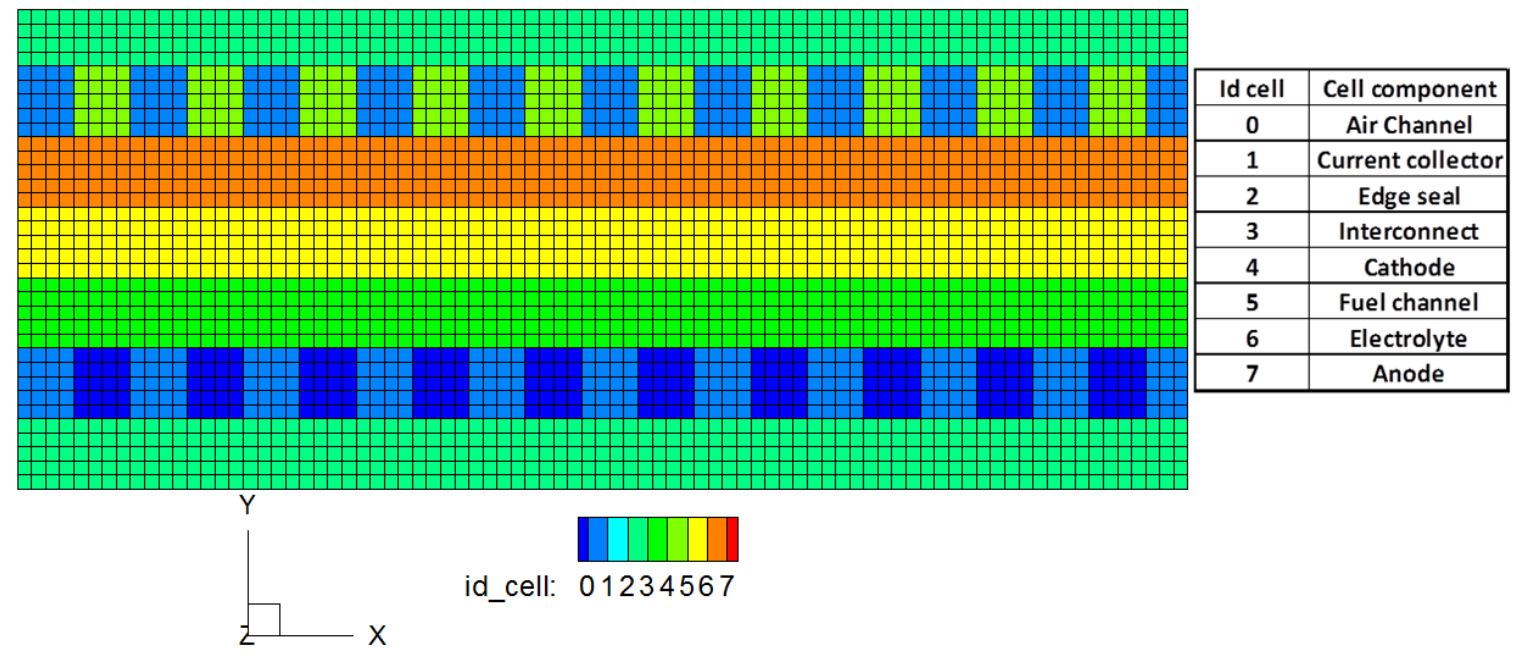

Figure 5.7: 3D-Schematic representation of the computational domain for three-dimensional planar SOFCs with 10 channels.

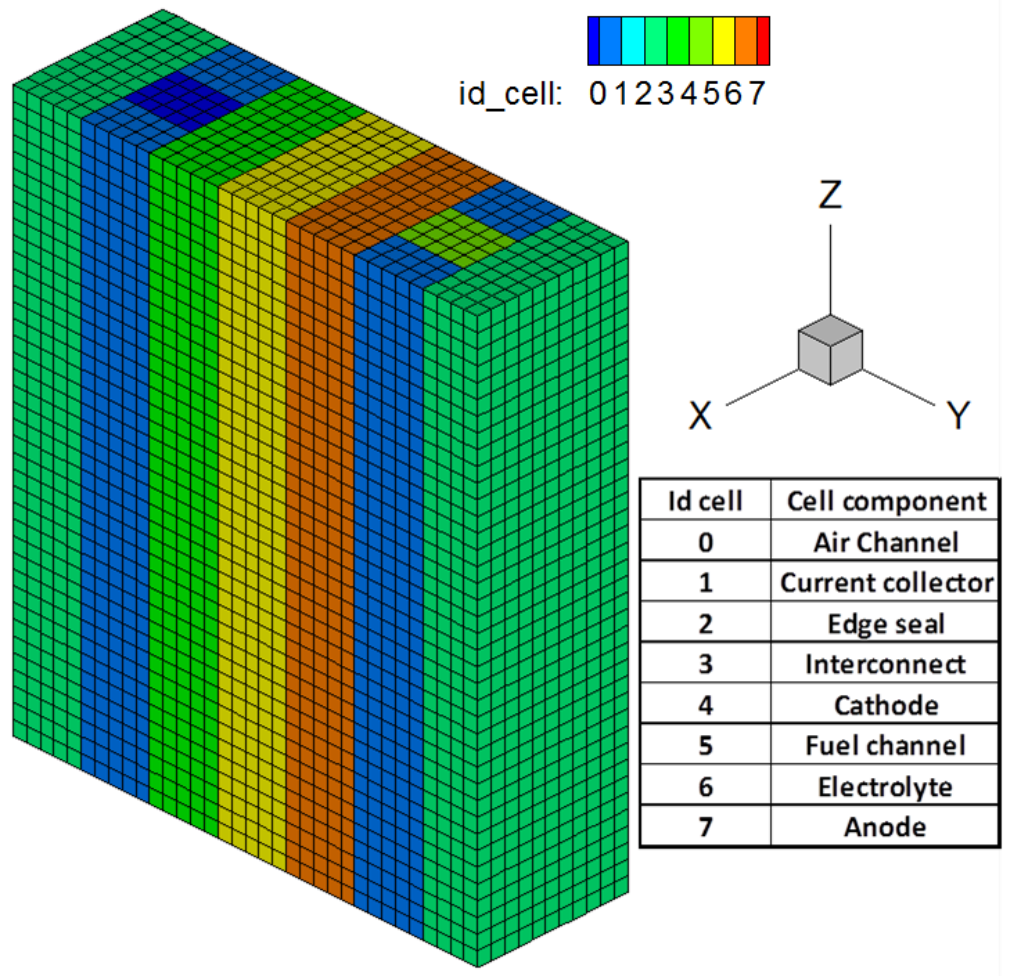

Figure 5.8: 3D-Schematic representation of the computational domain for three-dimensional planar SOFCs with one channel. 


\subsection{Results and discussions: Chapter 5}

\subsubsection{Simulations with ten fuel and ten air channels}

The transient 3-D computational code, Dream SOFC, is used to predict the temperature, species and current distribution inside a large SOFC with 10 air/fuel channels. The SOFC is utilized with dry hydrogen at $800{ }^{\circ} \mathrm{C}$ under $0.1 \mathrm{~A} / \mathrm{cm}^{2}$. Figure 5.9 shows the predicted transients of overall cell voltage (colored by green) and temperature (colored by red) at a monitoring point in the center of the anode active layer. Initially there is a slight increase in the cell voltage as the cell temperature increases due to heat produced inside the cell. After the temperature reaches a steady state, the cell potential remains constant. The contours of current density at anode electrolyte (top) and cathode electrolyte (bottom) interfaces shown in (Figure 5.10) show that there is a periodic variation in the channel-current collector alignment direction. This is because the current follows higher electrical conductor paths. The current distribution at cathode active layer shows slightly different behavior than that of anode active layer. This is due to the low air utilization inside the air channel. Low air utilization is used to decrease the cell temperature. The contours of current density at inlet (top) and out let (bottom) cross sectional interfaces (Figure 5.11) show that the magnitude of the current density is higher at the inlet than the outlet. This is due to the variation of the fuel and air utilizations along the channels. Contours of hydrogen and oxygen mass fraction at anode/electrolyte interface (top) and cathode/electrolyte interface (bottom) at steady state are shown in Figure 5.12. Similar periodic variation along channel-current collector direction is observed for hydrogen mass fraction whereas, a sharp periodic variation is observed for oxygen

mass fraction. This is due to the relatively higher diffusion coefficient of the hydrogen. It should be noted that this variation is not due to the periodic variation of current density at active layer. In this simulation the variations of the properties (temperature, current density, and species) from 
channel to current collector were small from channel to channel, which are only noticeable in the first and last channel (Figure 5.10-5.12). Therefore, in order to predict the phosphine induced performance degradation of a large planar cell with 10 channels, a planar cell with one channel is exposed to phosphine in the subsequent section.

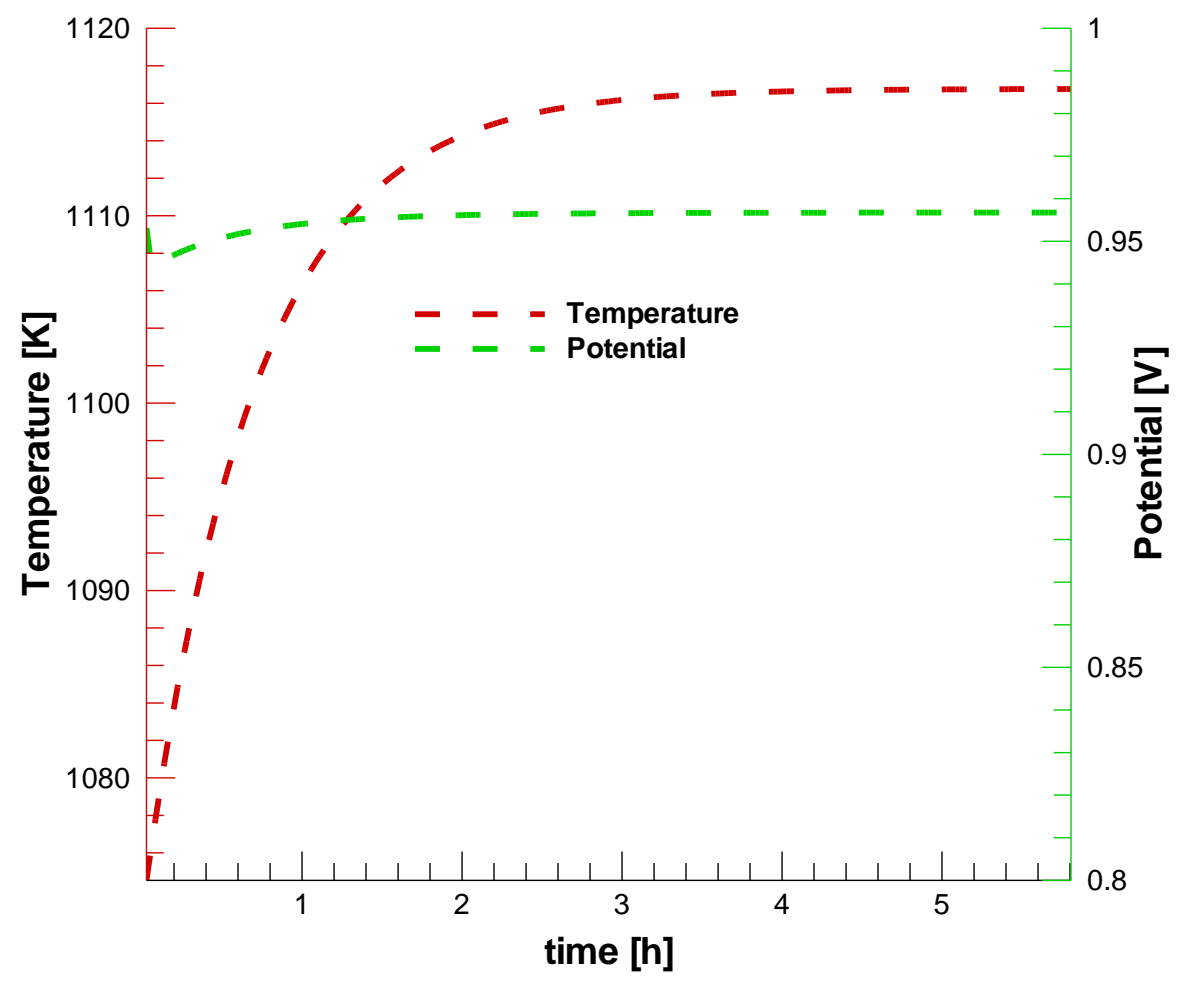

Figure 5.9: Transient variation of temperature (colored by red) and potential (colored by green) 

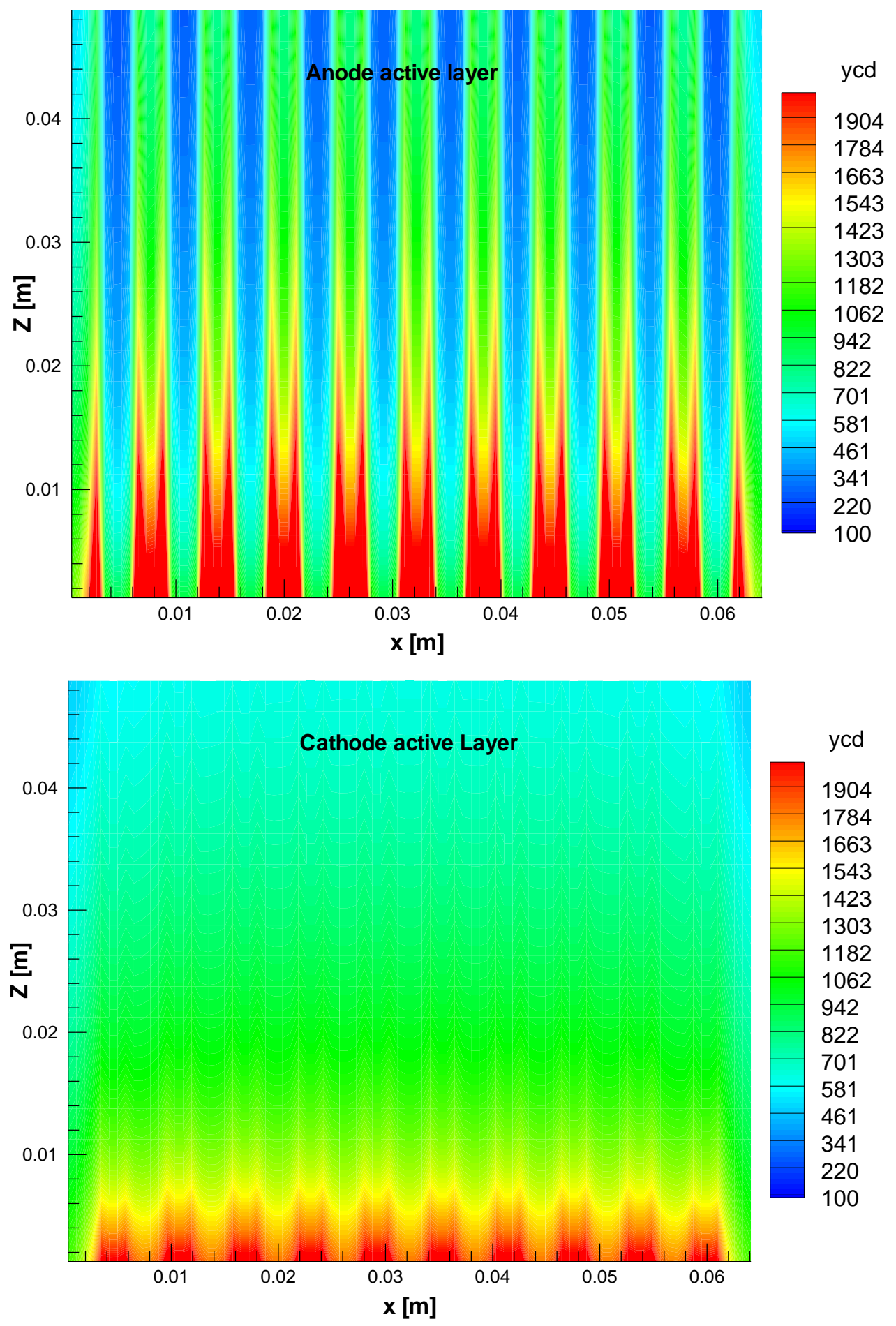

Figure 5.10: Contours of current density at anode/electrolyte interface (top) and cathode/electrolyte interface (bottom) at steady state without phosphine exposure 

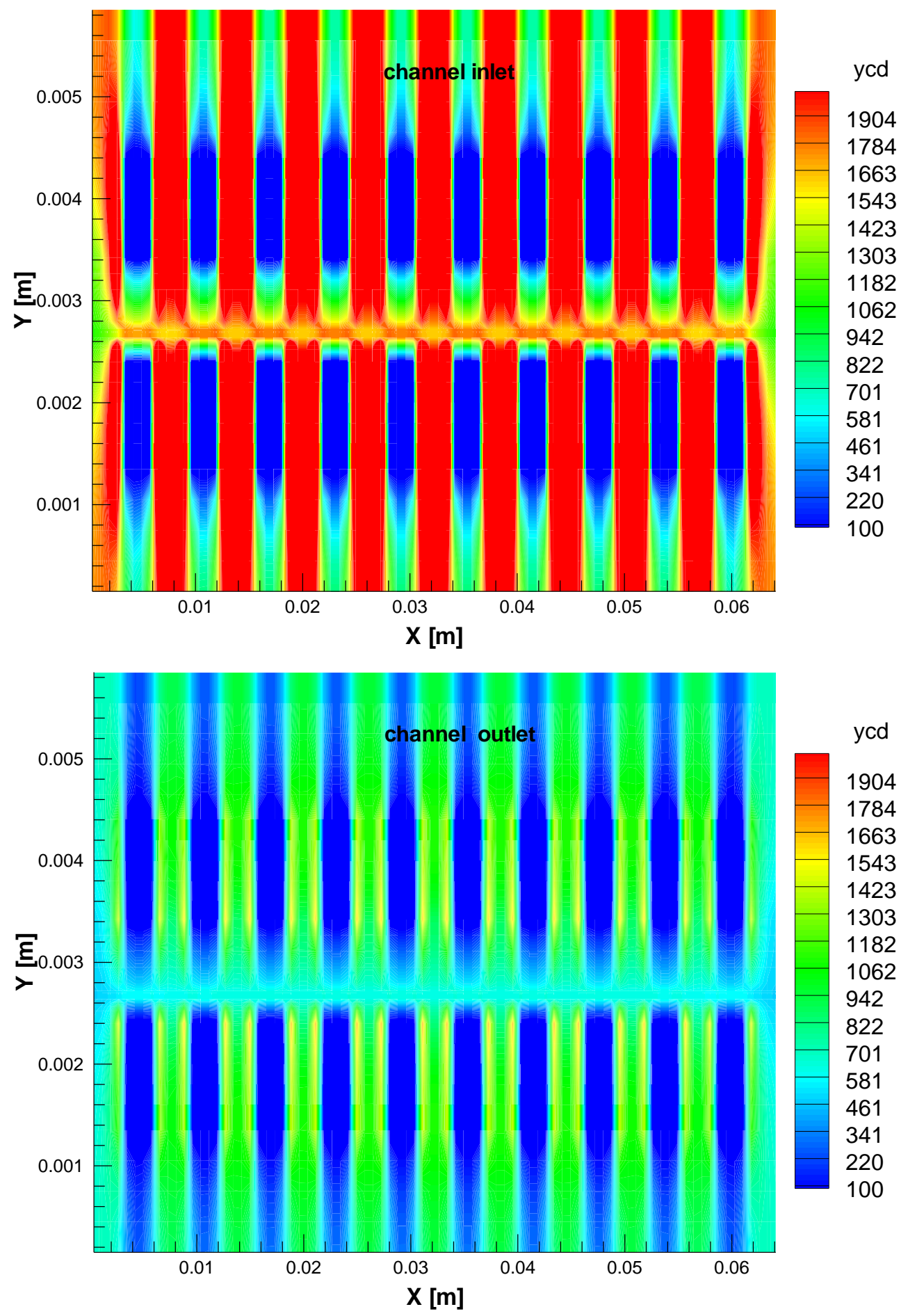

Figure 5.11: Contours of current density $\left(\mathrm{A} / \mathrm{cm}^{2}\right)$ at inlet (top) and at outlet (bottom) in steady state without phosphine exposure 

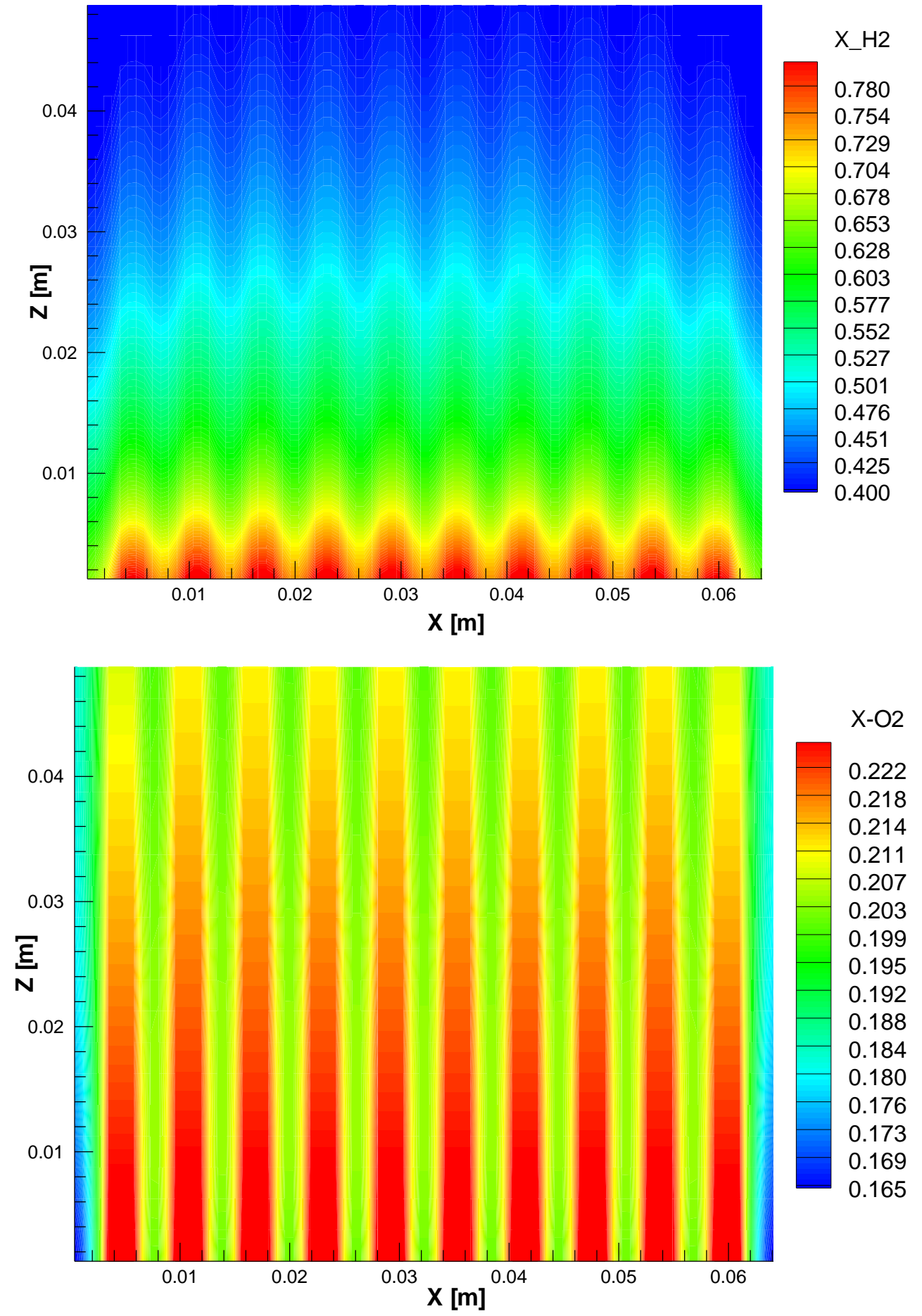

Figure 5.12: Contours of hydrogen and oxygen mass fraction at anode/electrolyte interface

(top) and cathode/electrolyte interface (bottom) respectively at steady state without phosphine exposure 


\subsubsection{Simulations with one air and one fuel channel}

A mixture of hydrogen, steam and phosphine was supplied to the anode side, and air was supplied to the cathode side. The computational domain (see Figure 5.8) is chosen such that it represents a practical repeat unit, containing one air and one fuel channel, in a planar cell of length $5 \mathrm{~cm}$. The choice of the computational domain thus makes the simulations more manageable in terms of computer time and memory while providing accurate predictions for a representative repeat unit of the cell. Figure 5.13(top) shows the predicted history of voltage produced by the cell operating at $0.1 \mathrm{~A} / \mathrm{cm}^{2}$ with dry hydrogen containing $10 \mathrm{ppm} \mathrm{PH}_{3}$ as fuel. Initially, there is a slight increase in the cell voltage as the cell temperature increases due to heat produced inside the cell. After that there is a steady state behavior until phosphine exposure. Further, once anode is exposed to phosphine, there is a decrease in the cell voltage due to the degradation caused by phosphine. The voltage dropped by $32 \%$ within $6.4 \mathrm{~h}$. Beyond that time the simulation became unstable due to instabilities in hydrogen transport equation. These instabilities exist due to the decrease in the hydrogen diffusion coefficient, which is altered by the impurity coverage. The coverage variation at a monitor point in the anode active layer, after phosphine exposure is shown in Figure 5.13 (bottom). Figure 5.14 and Figure 5.15 show the distribution of hydrogen and oxygen mass fractions, respectively, at different stages of the degradation process. Initially, hydrogen is being consumed near the inlet. As the porosity and the activity changes by increasing nickel deactivation and secondary phase formation, utilization near the inlet decreases and shifts downstream. This in turn, causes redistribution of oxygen on the cathode side. 

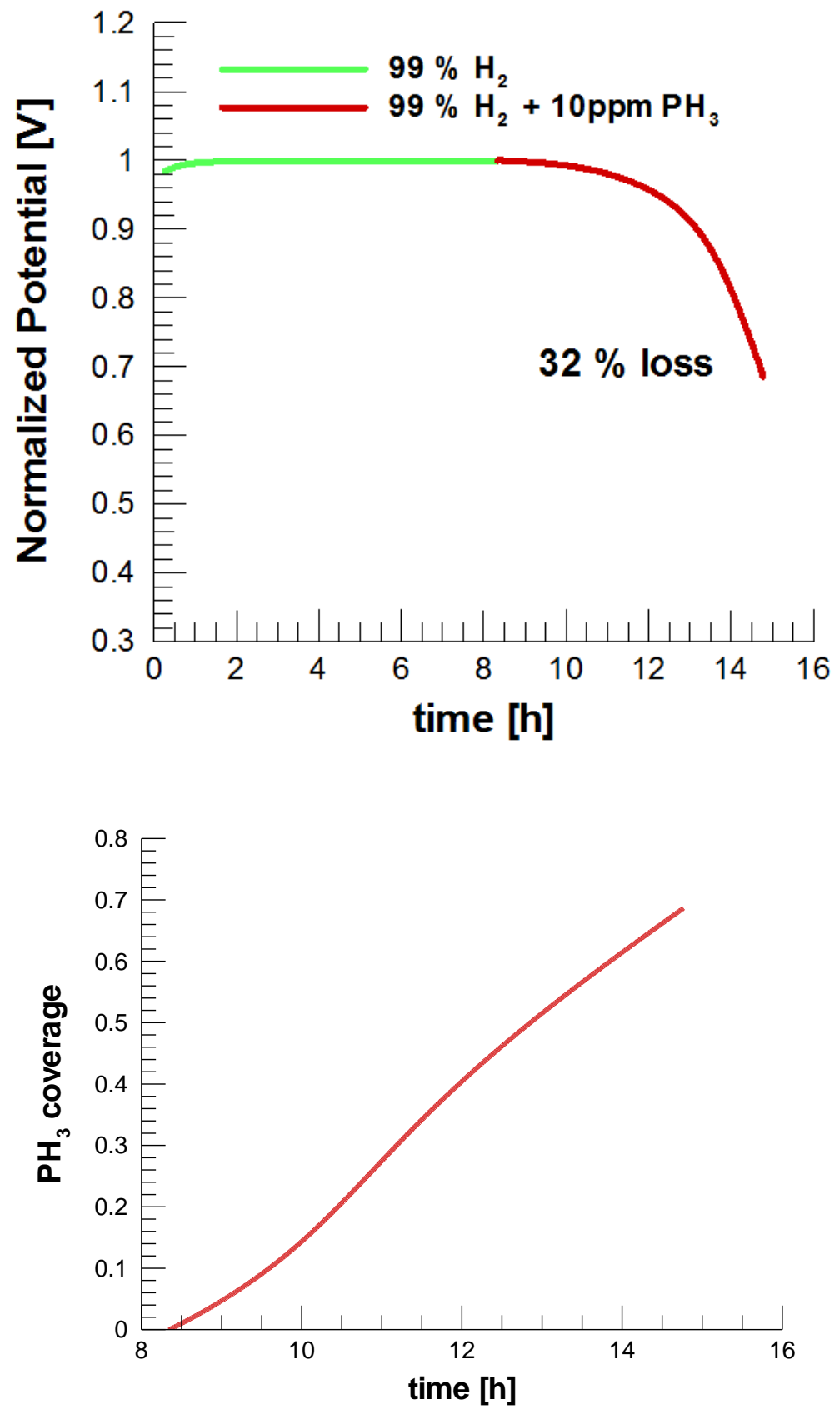

Figure 5.13: Cell voltage drop (top) and phosphine coverage formation at electrolyte/anode interface (bottom) during phosphine induced performance degradation 
The anode regions directly underneath the inlet region are initially exposed to the phosphine introduced in the fuel and the active nickel sites in that region are thus consumed first by phosphine attack. This can be seen from contours of $\mathrm{PH}_{3}$ coverage at anode/electrolyte active interface in Figure 5.16 for three different degradation stages. It is seen that the $\mathrm{PH}_{3}$ coverage in the regions underneath the inlet region is much higher compared to that in the regions near the outlet and the coverage is also increasing with time. As the coverage of $\mathrm{PH}_{3}$ builds up near the active interface it reduces the electrochemical activity of those regions. In addition, the coverage also decreases (by design of the model) the porosity of the affected regions and reduces the effective gas diffusion coefficient. The local charge transfer kinetics are thus altered by the $\mathrm{PH}_{3}$ coverage and the current distribution inside the cell changes with time as coverage distribution evolves with time. Figure 5.17 shows the current density distribution at the active anode/electrolyte interface at four different stages. It can be seen from Figure 5.17 that during the initial stages there is relatively high charge transfer rates in the regions underneath the channel inlet, but as the electrochemical activity is diminished due to contaminant coverage, the current densities in this region are reduced. Consequently, in order to maintain the applied constant average current density, the current is redistributed and the local current densities increased with time in the regions underneath the ribs and channel outlet. This redistribution, however, is not sustainable since the regions underneath the rib are hard to replenish with reactants especially when the effective gas diffusivity in the regions underneath the channel is lowered due to contaminant coverage. 

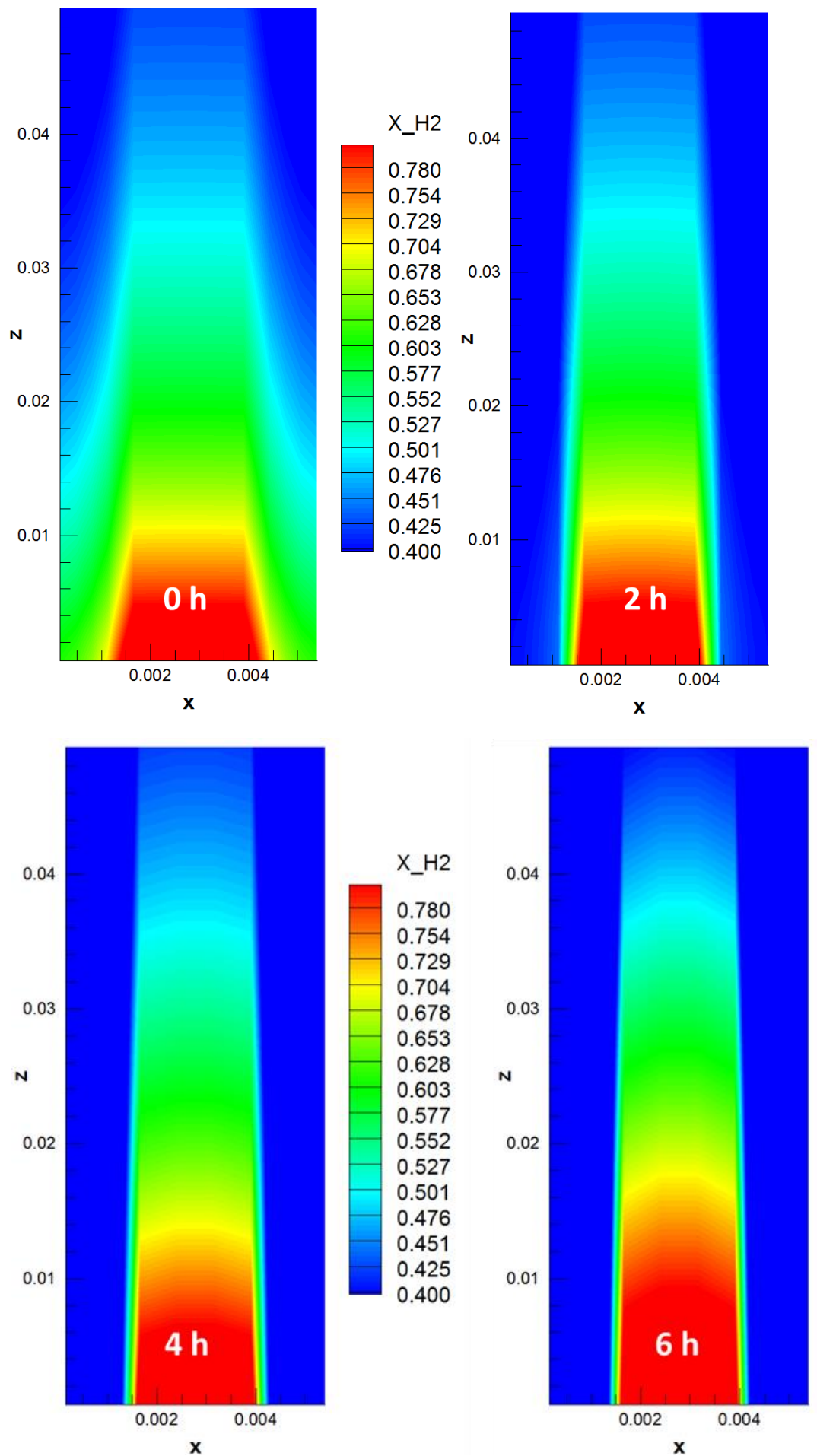

Figure 5.14: Contours of hydrogen mass fraction at anode/electrolyte interface at steady state (top-left) after $2 \mathrm{~h}$ (top-right), $4 \mathrm{~h}$ (bottom-left) and $6 \mathrm{~h}$ (bottom-right) of phosphine exposure 

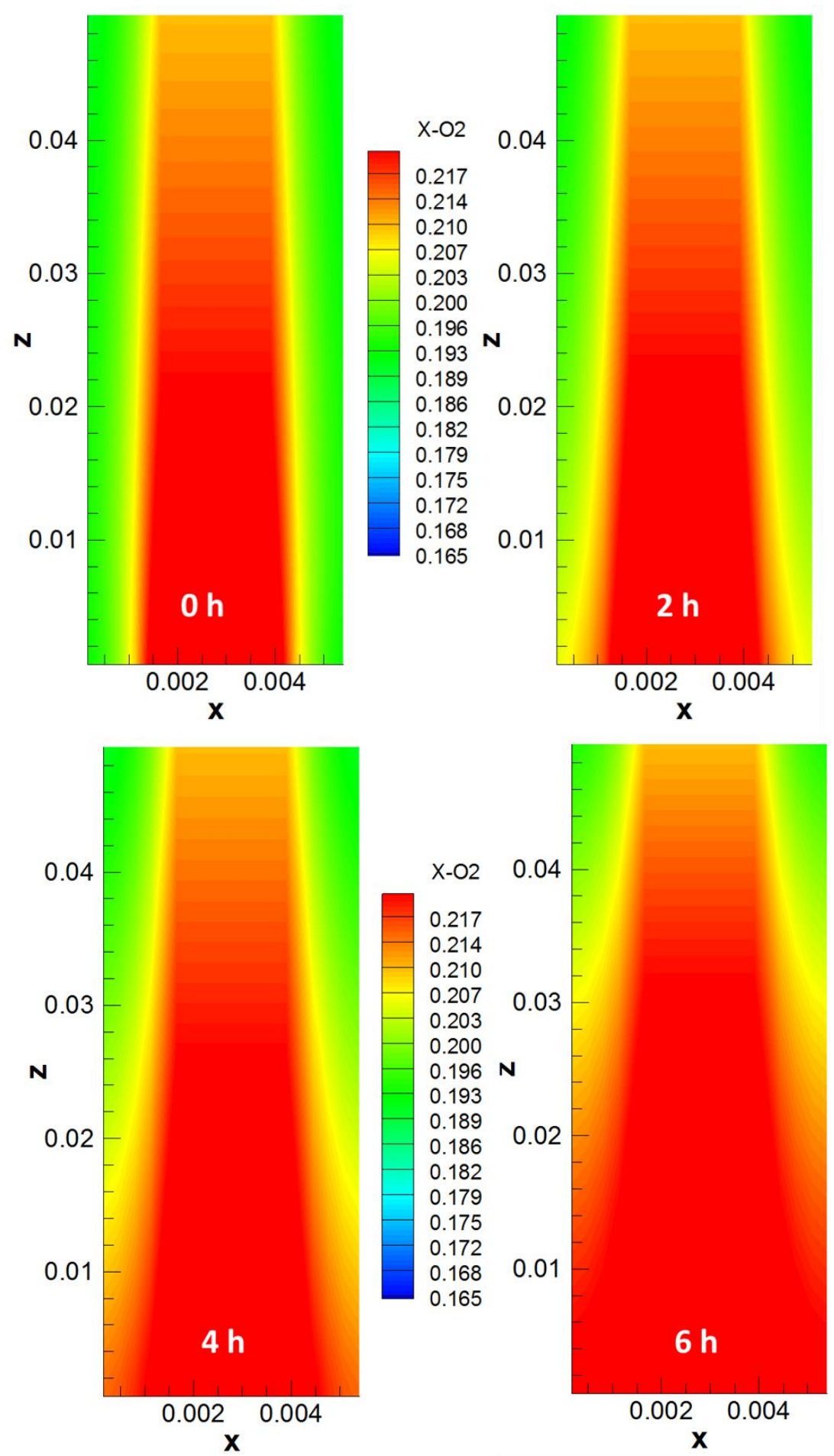

Figure 5.15: Contours of oxygen mass fraction at cathode/electrolyte interface at steady state (top-left) after $2 \mathrm{~h}$ (top-right), $4 \mathrm{~h}$ (bottom-left) and $6 \mathrm{~h}$ (bottom-right) of phosphine exposure 

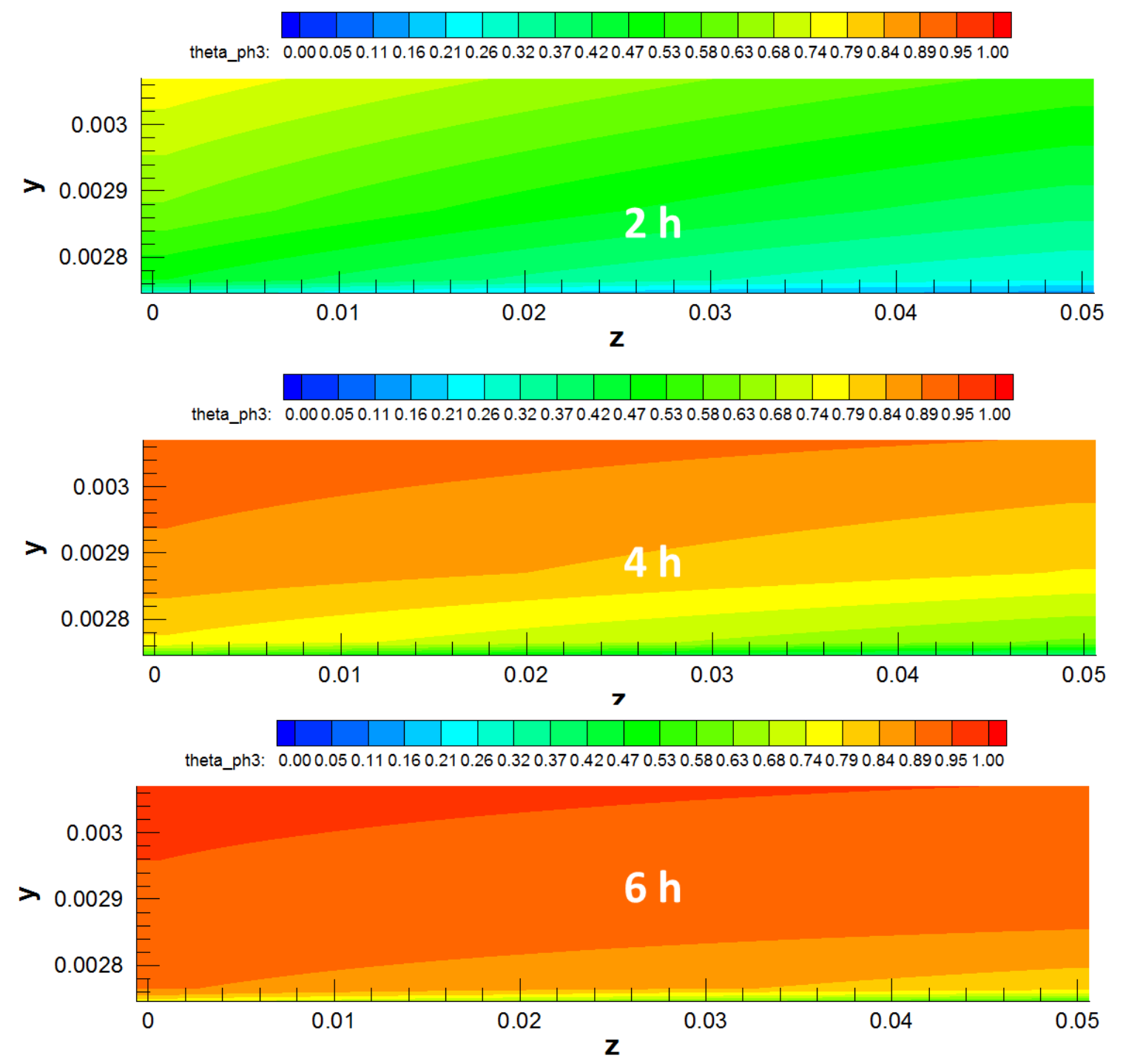

Figure 5.16: Contours of phosphine coverage at $\mathrm{x} / 2$ ( $\mathrm{y}-\mathrm{z}$ plane) after $2 \mathrm{~h}$ (top), $4 \mathrm{~h}$ (middle) and $6 \mathrm{~h}$ (bottom). 

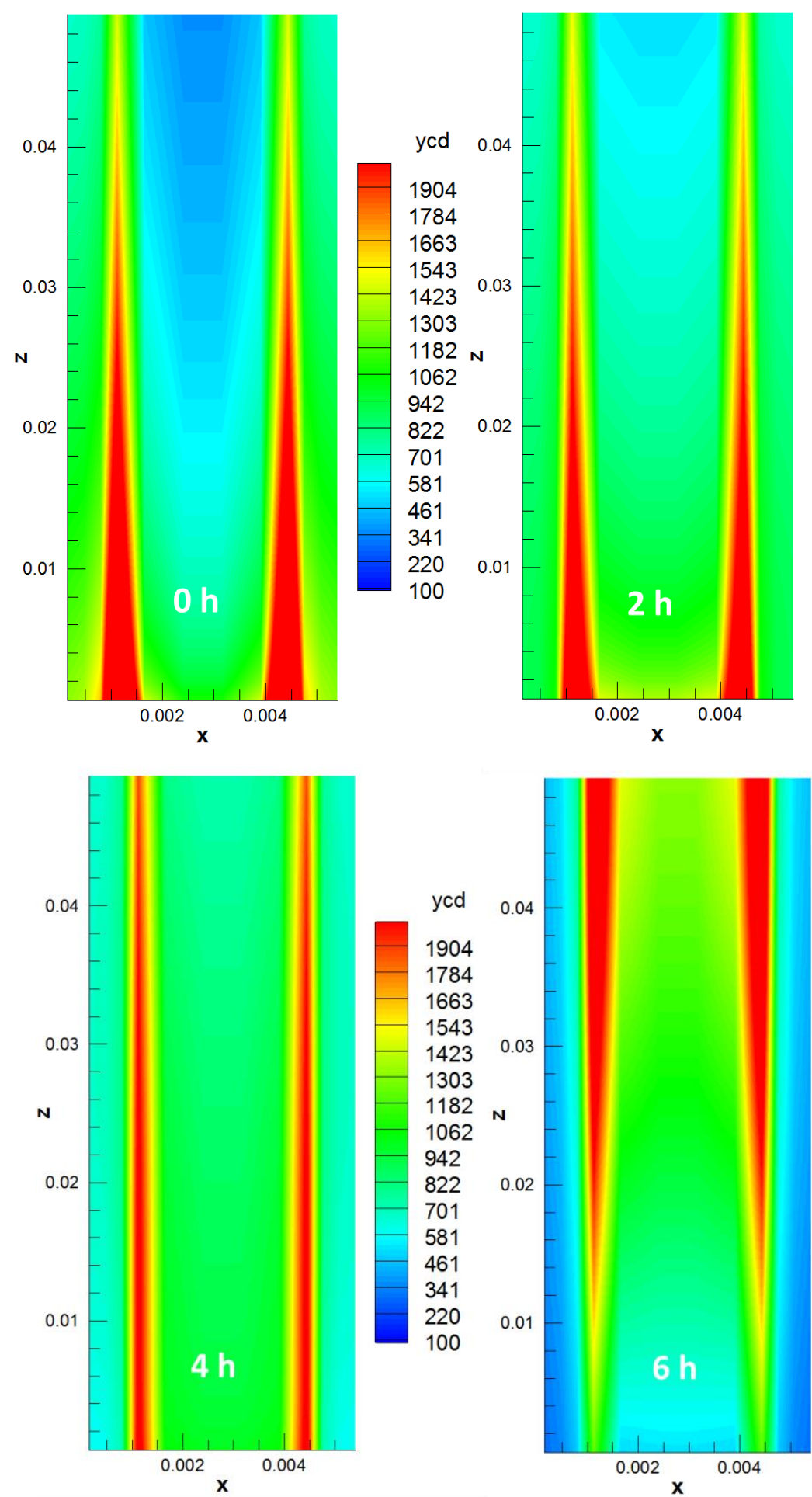

Figure 5.17: Contours of current density $\left(\mathrm{A} / \mathrm{m}^{2}\right)$ at anode/electrolyte interface at steady state (top-left) after $2 \mathrm{~h}$ (top-right), $4 \mathrm{~h}$ (bottom-left) and $6 \mathrm{~h}$ (bottom-right) of phosphine exposure 
As depicted in Figure 5.14, the hydrogen is also re-distributed at the anode/electrolyte interface as the degradation progresses. The simulation thus becomes unstable (indicative of cell malfunctioning) when insufficient reactants are available in the regions where there is high electrochemical activity (Figure 5.14 and Figure 5.17). In the current simulations the occurrence of insufficient reactants is handled by re-scaling the electrochemical potential in the starving computational cells using the ratio of available hydrogen to hydrogen required for the predicted charge transfer rate in the cell. In reality, however, the oscillations observed in current simulations could also mean that the cell experiences a catastrophic failure about the time at which the oscillations have started. In a real cell, if some regions become starved of hydrogen, the charge transfer redistributes to other regions. But in the current simulations, the regions where hydrogen is available are electrochemically inactive (due to contaminant coverage) and thus the cell may not be able to produce the applied current causing the voltage to drop suddenly. 


\subsubsection{Electrochemical evaluation of the degraded cell}

In this sub section, additional simulations are performed to diagnose the gradually degraded cell by analysis of impedance and voltage-current behavior at various degradation levels. The planar cell conductivity varies with time during the transient simulations. This is due to the important role of the ohmic losses in resulting V-I curves and impedance. In planar cell simulations, the electrical conductivity and the ionic conductivity of the cell components have functional dependency on temperature. Since the temperature changes with time during phosphine exposure, these properties are altered by the temperature variation. The influence of the temperature on each cell component conductivity is given as follows;

$$
\begin{aligned}
& \sigma_{N i-Y S Z}^{e}=-0.38 T+824.97 \quad \text { Anode } \\
& \sigma_{L S M-Y S Z}^{e}=0.0254 T-1.7542 \quad \text { Cathode } \\
& \sigma_{Y S Z}^{i}=A \exp \left(-\frac{E_{a}}{R T}\right) \quad \text { Electrolyte }
\end{aligned}
$$

Where $\sigma, i$ and $e$ indicate conductivity, ionic and electronic, respectively. The pre-exponential coefficient $\mathrm{A}$ and the activation energy are given as 61456 Siemens $/ \mathrm{m}$ and $85634.2 \mathrm{~J} / \mathrm{mole}$, respectively. The curious readers for these functional dependencies are referred to the $\mathrm{PhD}$ thesis by Elizalde-Blancas [53].

V-I curves at instant degradation levels: In order to assess the electrochemical behavior of the cell, additional simulations are performed to estimate V-I behavior of one channel planar cell at 
steady state condition without trace element exposure and with the trace element exposure at different instants. To produce a V-I curve, simulations were started with $0.005 \mathrm{~A} / \mathrm{cm}^{2}$ current density and continued with increment of $0.01 \mathrm{~A} / \mathrm{cm}^{2}$ until limiting current is reached. The V-I behavior of the cell predicted by the additional simulations performed with DREAM-SOFC code for different degradation times are shown in Figure 5.18. The V-I curves obtained at different times of phosphine exposure clearly show that while phosphine coverage increases (Figure 5.16), the polarization resistance increases, slowly at first and sharply after some time. Additionally, Figure 5.18 also indicates that the initial changes in polarization resistance with exposure time were mostly due to increase in activation losses since the slope of VI at low current densities is increasing. Further, due to the current re-distribution during gradual degradation, the temperature redistributes accordingly. This change in temperature alters (usually decreasing) the ohmic resistance. Therefore, the changes in the V-I curves are mostly due to the activation over potentials. This is consistent with the button cell simulations. However, it is important to mention that in button cell simulations temperature is assumed to be constant at all times. Additional simulations were performed to analyze the polarization behavior of the cell during phosphine degradation along the channel in different region of the cell (See Appendix A). 


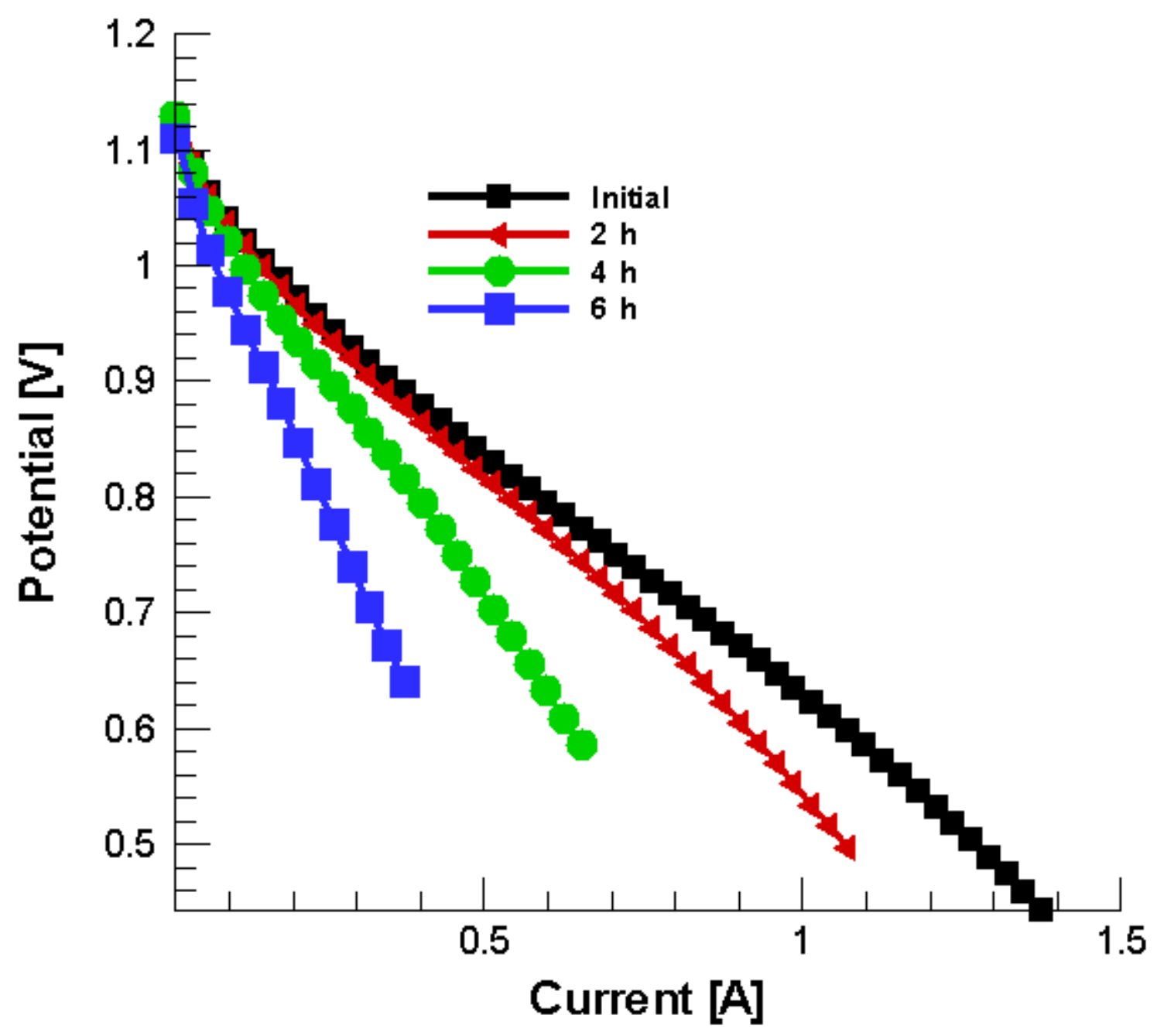

Figure 5.18: Predicted V-I curves of planar cell at different times of phosphine exposure for dry hydrogen. 


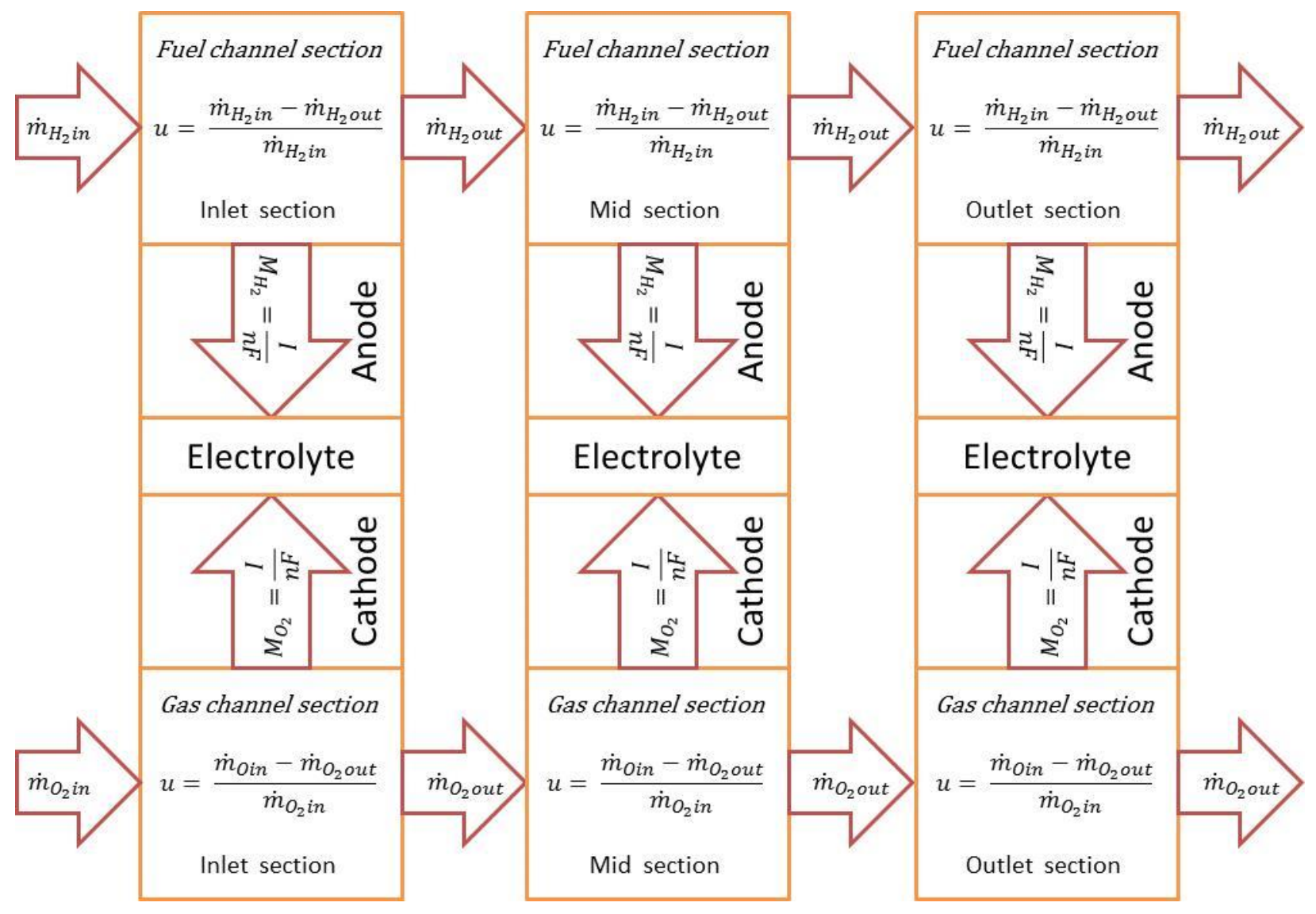

Figure 5.19: Planar cell partitioning for detailed impedance analysis 
Impedance response at instant degradation levels: Impedance analysis is carried out by performing additional simulations using DREAM-SOFC. Due to the variation in species distribution inside the fuel channel, the degradation patterns of the planar cell show differences along the fuel flow direction inside the channel. Therefore, in order to analyze the electrochemical behaviors of the planar cell exposed to phosphine, the cell impedance is calculated for the whole geometry and also for three different divisions of the cell as shown in Figure 5.19.

The impedance simulation approach used in this study is similar to the one presented by Bessler's rapid impedance modeling [64]. The impedance simulations are based on the transient numerical simulation of DREAM-SOFC; microsecond current step followed by the potential step. The current excitation and potential relaxation process is carried out by the following equations;

$$
\begin{gathered}
I(t)=I_{\text {base }}-\left[1-\exp \left(-\frac{t}{\tau}\right)\right] I_{\text {step }} \\
I_{f}=-(I(t)-I(t=0)) \\
\left.E_{f}=E(t)-E(t=0)\right)
\end{gathered}
$$

Where $I_{\text {base }}$ is the total cell current before the current excitation and $I_{\text {step }}$ is the magnitude of the step which is taken $1 \%$ of the $I_{\text {base }} . \tau$ is defined as the relaxation time, which is chosen as $10^{-5} \mathrm{~s}$. The time step used for the transient impedance simulations was $10^{-6}$. The simulations were performed for $3 \mathrm{~s}$. Finally, the calculated time trace of the exciting current, $I_{f}$, and the potential relaxation, $E_{f}$, are calculated from Eq. (5.7) and as in Figure 5.20 for whole cell. The time traces of exciting current and potential relaxation for the different regions during phosphine degradation are presented in Appendix A. Further, the calculated transient current-voltage values are reconstructed by applying a discrete fast Fourier transform (DFFT) for uniformly spaced points in the time and frequency domain. 


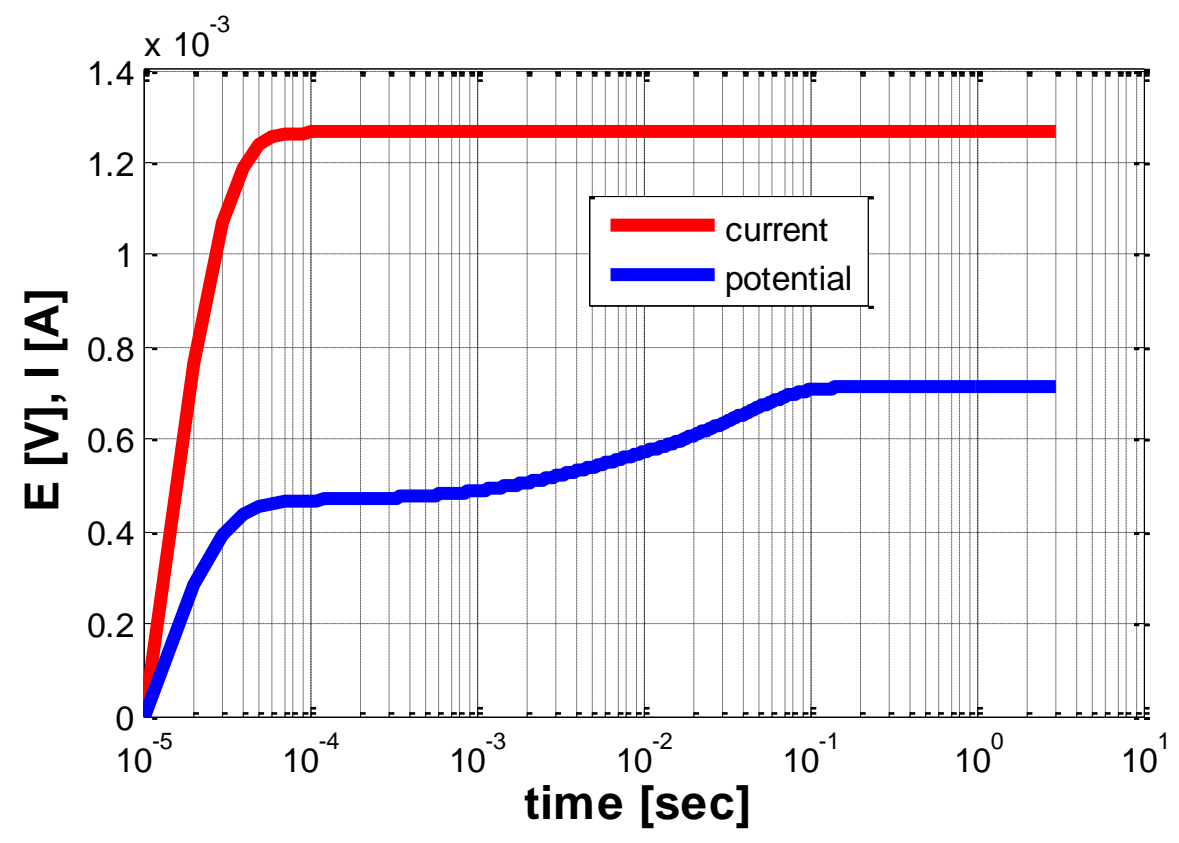

Figure 5.20: Time trace of exciting current and potential relaxation (exponential step $\boldsymbol{\tau}=$ $\mathbf{1 0}^{-5}$ ) for impedance analysis of planar cell simulated by DREAM-SOFC

The impedance response of the one channel planar cell for the whole cell geometry and the different parts of the cell during different times of phosphine exposure shown in Figure 5.21-24. The impedance of the cell without phosphine exposure, by Nyquist (top) and Bode (bottom) representations are shown in the Figure 5.21. As it can be seen from the impedance curves, the overall cell resistance, ohmic and polarization, is smaller than that of the cell inlet middle and outlet sections. This is an expected behavior. For the ohmic resistance, cell sections have lower ionic and electronic conductivities due to the lower current effective area than that of the whole cell geometry. It can be consider that the cell sections are connected parallel to form complete cell. The impedance of a parallel connected circuit is the reciprocal of the sum of the reciprocals of the impedance of each circuit elements and it can be calculated from Eq 8 . 


$$
Z_{\text {cell }}=\frac{1}{\frac{1}{Z_{\text {in }}}+\frac{1}{Z_{\text {mid }}}+\frac{1}{Z_{\text {out }}}}
$$

In order to assess the validity of the sectional impedance calculations Eq. 5.8 is used as a test. The results shown in Table 5.3 indicated that the impedance calculations by cell portioning are in good agreement with the one calculated from the simulation of the whole cell.

Table 5.3: Roughly impedance calculations at low and high frequencies

\section{Impedance}

\begin{tabular}{lccccc}
\hline & Inlet & Middle & Outlet & $\begin{array}{c}\text { equivalent } \\
\text { impedance }\end{array}$ & Cell \\
\hline Low frequency & 1.44 & 1.58 & 1.77 & 0.53 & 0.50 \\
High frequency & 0.92 & 0.92 & 0.89 & 0.30 & 0.29 \\
\hline
\end{tabular}

In Figure 5.21, the impedance curves from inlet to outlet show that the impedance decreases in flow direction. Actually, this is due to the decrease in fuel utilization and the temperature increase along the fuel and air channels. Fuel utilization along the channel is directly proportional to the slope of mole fraction of hydrogen curve along the channel. The mole fraction of the hydrogen at different times of phosphine exposure is shown in Figure 5.25. As it can be seen in Figure 5.25, the slope of hydrogen mole fraction curve at initial time of phosphine exposure is decreasing from inlet to outlet direction, hence the fuel (hydrogen) utilization behaves similarly. It is important to mention that this behavior is due to the current redistribution during phosphine exposure time. Higher utilizations increase the polarization resistance due to the fuel starvation at electrochemically active area. This increase in polarization resistance can be observed from Nyquist and Bode representation of the impedance at initial time of phosphine exposure (Figure 5.21). The polarization resistance is a function of the temperature and it decreases with increasing 
temperature. The temperature variation is due to the fuel and air utilization gap from inlet to outlet direction in the channels and the current redistribution. Usually, this difference in the temperature can be significant when the cell is operated with high utilizations. However, in this study, a small air and fuel utilization is used (Table 5.2). Even this small air and fuel utilization affects the temperature distribution along the channels. Therefore, the change in the impedance of the cell sections at the same degradation level is also due to the temperature variation at that instant degradation level.

As it is depicted in Figure 5.25, the fuel utilization increases from the inlet to outlet at further times $(6 \mathrm{~h})$ of the phosphine exposure due to the current redistribution inside the cell. However the impedance of the cell still decreases from inlet to outlet direction. This behavior can be explained by the phosphine coverage propagation inside the anode at different degradation levels. As it can be seen in Figure 5.16, phosphine coverage increases first at the inlet region, therefore the polarization resistance increases at this region.

As it is seen in Figure 5.21-24, the ohmic resistance varies in the different cell sections especially for long phosphine exposure time ( $4 \mathrm{~h}$ and $6 \mathrm{~h})$. This is due to the current redistribution, which yields the temperature redistribution inside the cell. Mathematical dependency of the electronic and ionic conductivities on the temperature leads to the variation of the ohmic resistance.

The temperature profiles at different times of the degradation along the fuel channel are depicted in Figure 5.25. It is seen that the temperature increases with increasing phosphine exposure time. Also Figure 5.25 indicates that the temperature variation from inlet to outlet of the channel at a degradation level is significant. The temperature difference between cell sections also increases as a function of the phosphine exposure time. Increasing the temperature gap between the cell 
increases the ohmic resistance changes between cell sections. The difference in ohmic resistance is significant at further time of the degradation. In addition, the overall cell ohmic resistance slightly varies during the degradation time. Such behavior is not possible to be observed in button cell degradation simulations. This is due to the constant temperature assumption in button cell degradation simulations.

Another important feature of the impedance analysis is the magnitude of the polarization resistance during the gradual degradation process. Total cell resistance, polarization and ohmic resistance can be calculated from V-I curves via Eq. 9 and the values are listed in Table 5.4

$$
\left.R_{\Omega}+R_{p}=\frac{d V}{d i}\right)_{I=0.278 A}
$$

Table 5.4: Polarization and ohmic resistance of the cell at operating current load in different times of degradation

\begin{tabular}{|c|c|c|c|c|}
\hline & $\Delta V$ & $\Delta I$ & $\begin{array}{c}R_{\Omega}+R_{p} \\
(V-I)\end{array}$ & $\begin{array}{c}R_{\Omega}+R_{p} \\
\text { (Impedance) }\end{array}$ \\
\hline initial & 0.013 & 0.027 & 0.48 & 0.50 \\
\hline 2 h & 0.014 & 0.027 & 0.52 & 0.53 \\
\hline 4h & 0.019 & 0.027 & 0.70 & 0.64 \\
\hline $6 h$ & 0.036 & 0.027 & 1.33 & 1.19 \\
\hline
\end{tabular}

As it is summarized in Table 5.4, the overall cell resistance increases with increasing phosphine exposure time. This trend is expected due to the phosphine propagation and poisoning effect on the SOFC's anode by the time of the degradation. As it can be seen in Figure 5.13 (top) the cell performance decreases as the anode is exposed to phosphine, therefore the cell resistant increases as a consequence of the phosphine poisoning. The overall cell resistance that is calculated with the 
impedance response at different degradation times is in good agreement with that of the polarization behavior. 

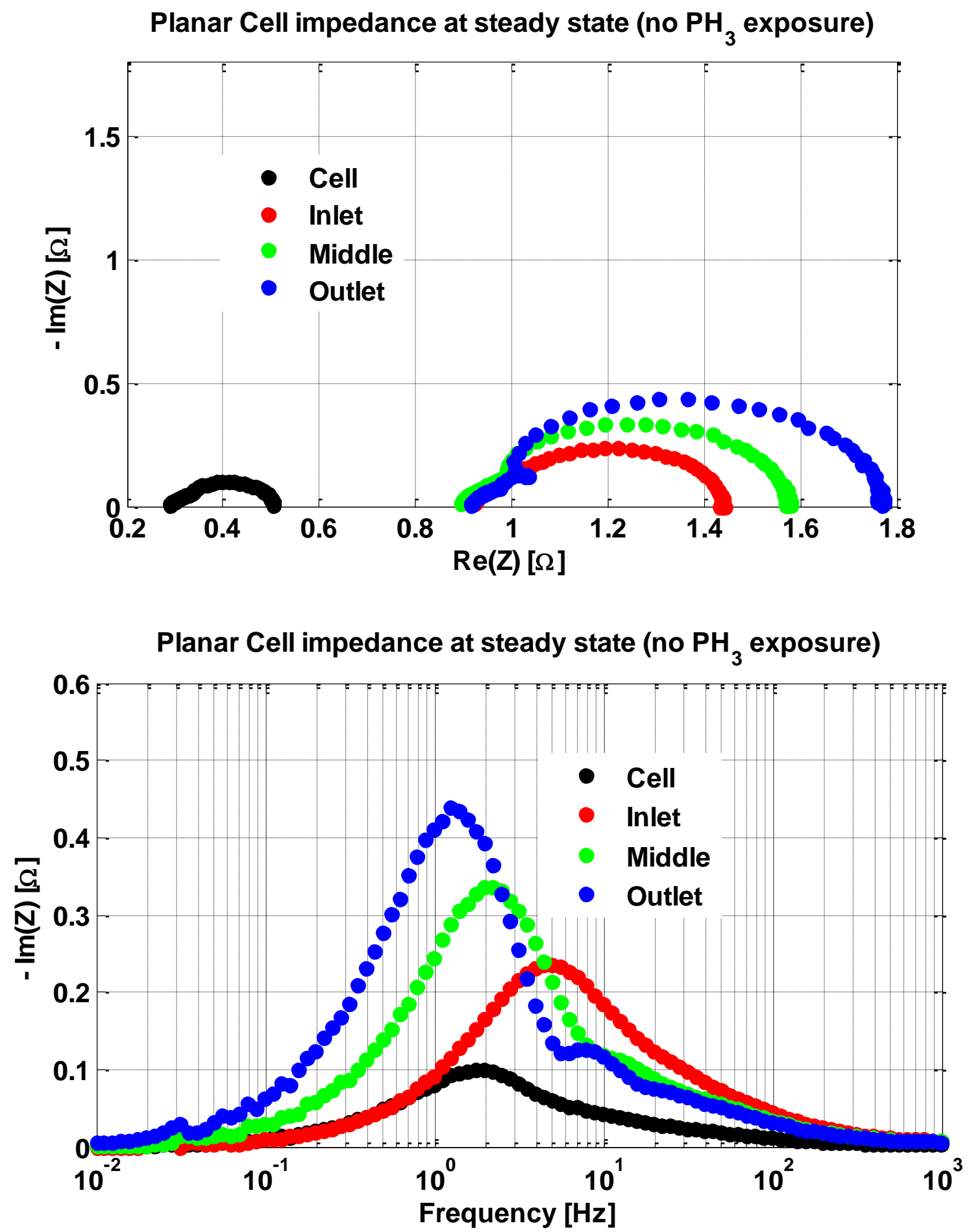

Figure 5.21: Predicted impedance curves for planar cell in different regions (inlet, middle and outlet) at steady state condition of the cell: Nyquist representation (top) and Bode representation (bottom) 


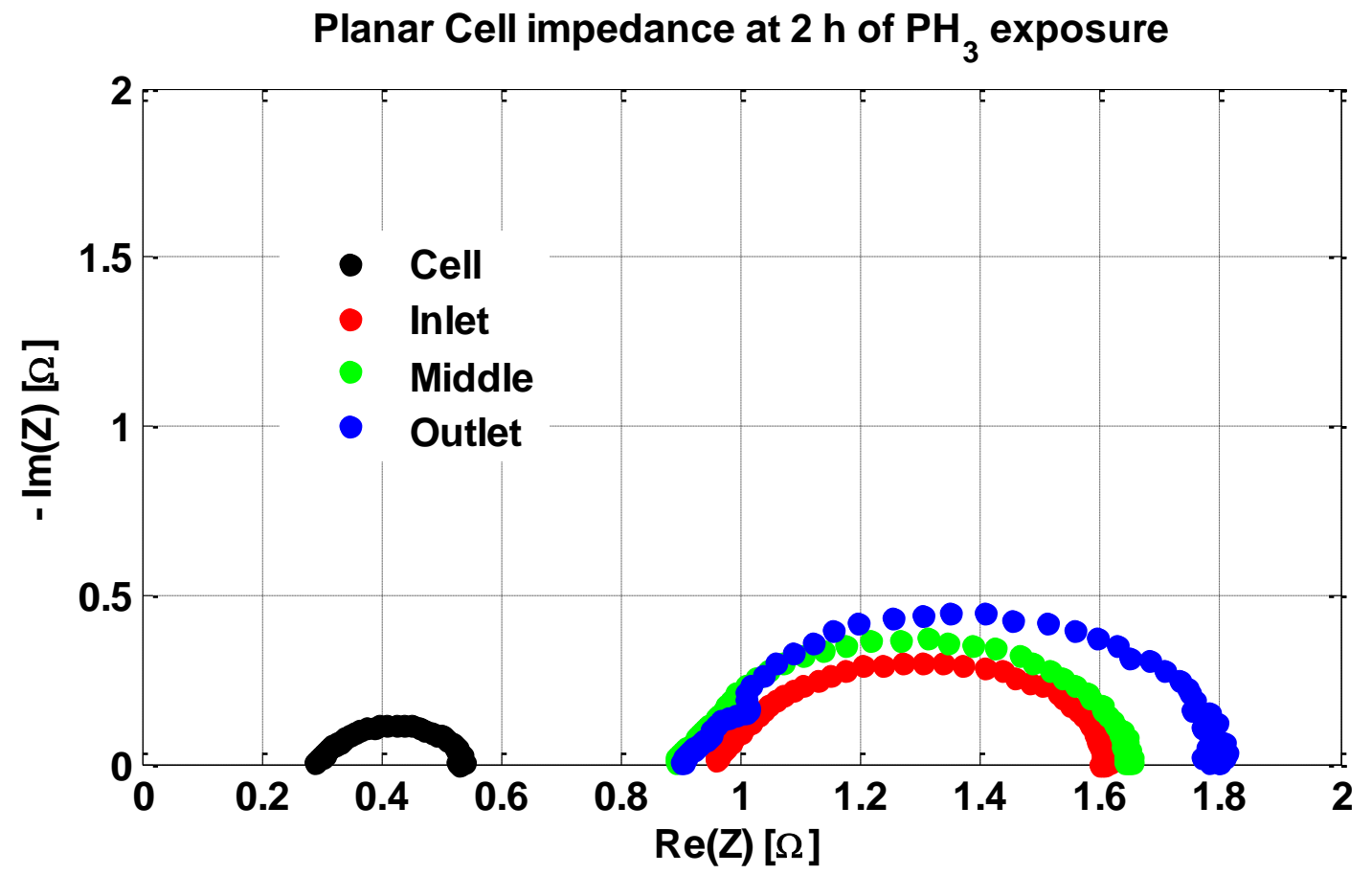

Planar Cell impedance at $\mathbf{2} \mathrm{h}$ of $\mathrm{PH}_{3}$ exposure

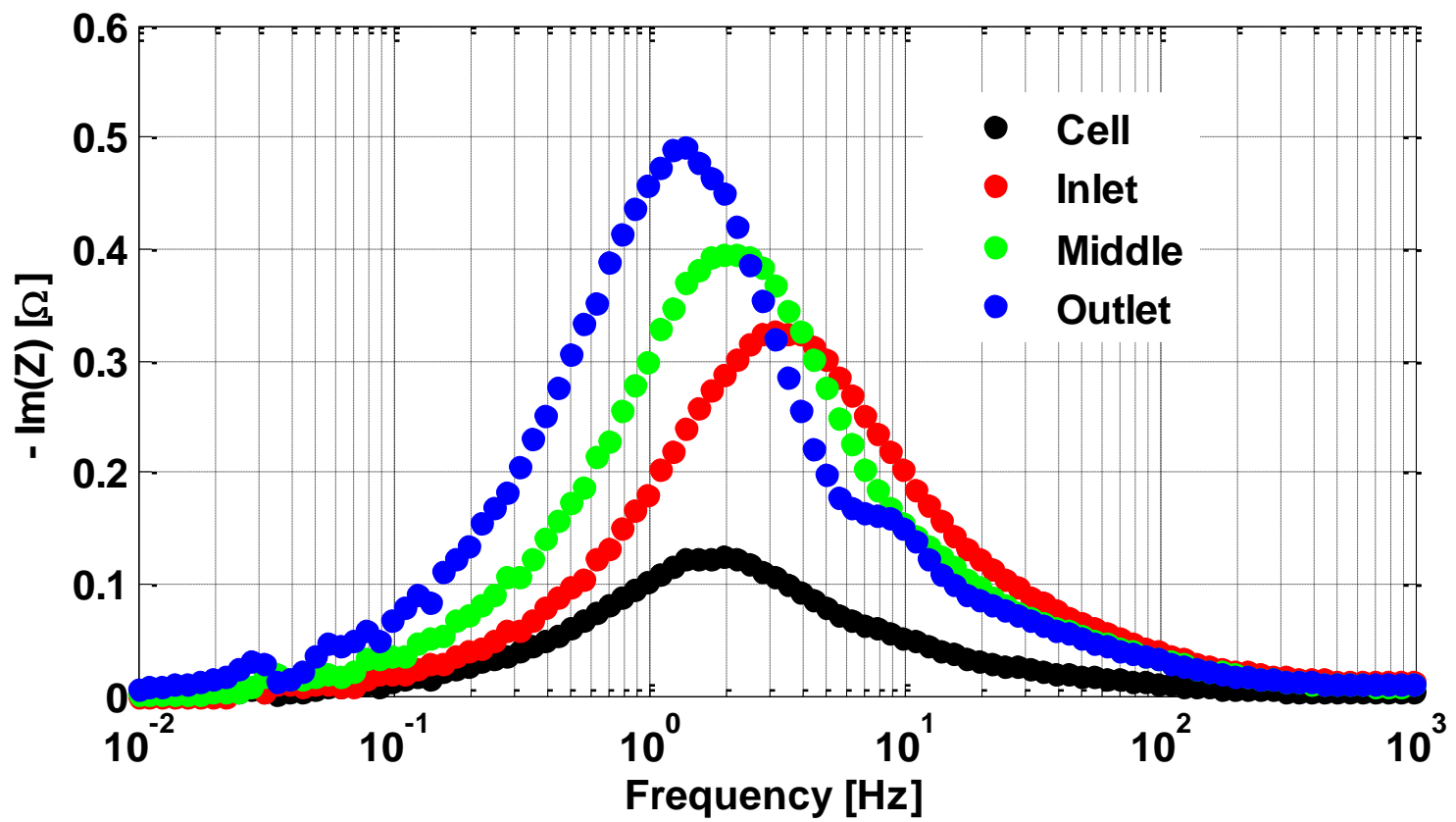

Figure 5.22: Predicted impedance curves for planar cell in different regions (inlet, middle and outlet) at $2 \mathrm{~h}$ of phosphine exposure: Nyquist representation (top) and Bode representation (bottom) 


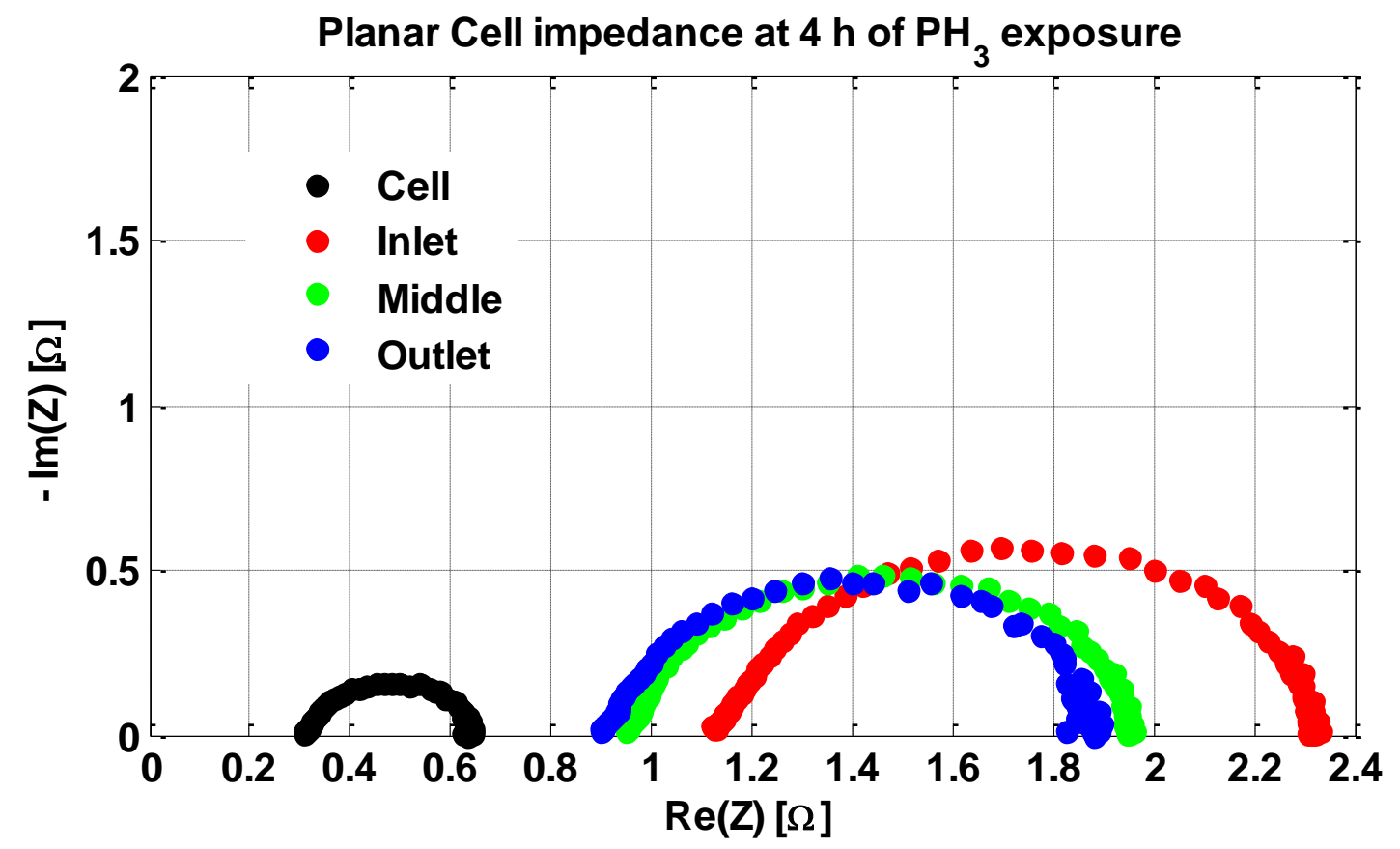

Planar Cell impedance at $4 \mathrm{~h}$ of $\mathrm{PH}_{3}$ exposure

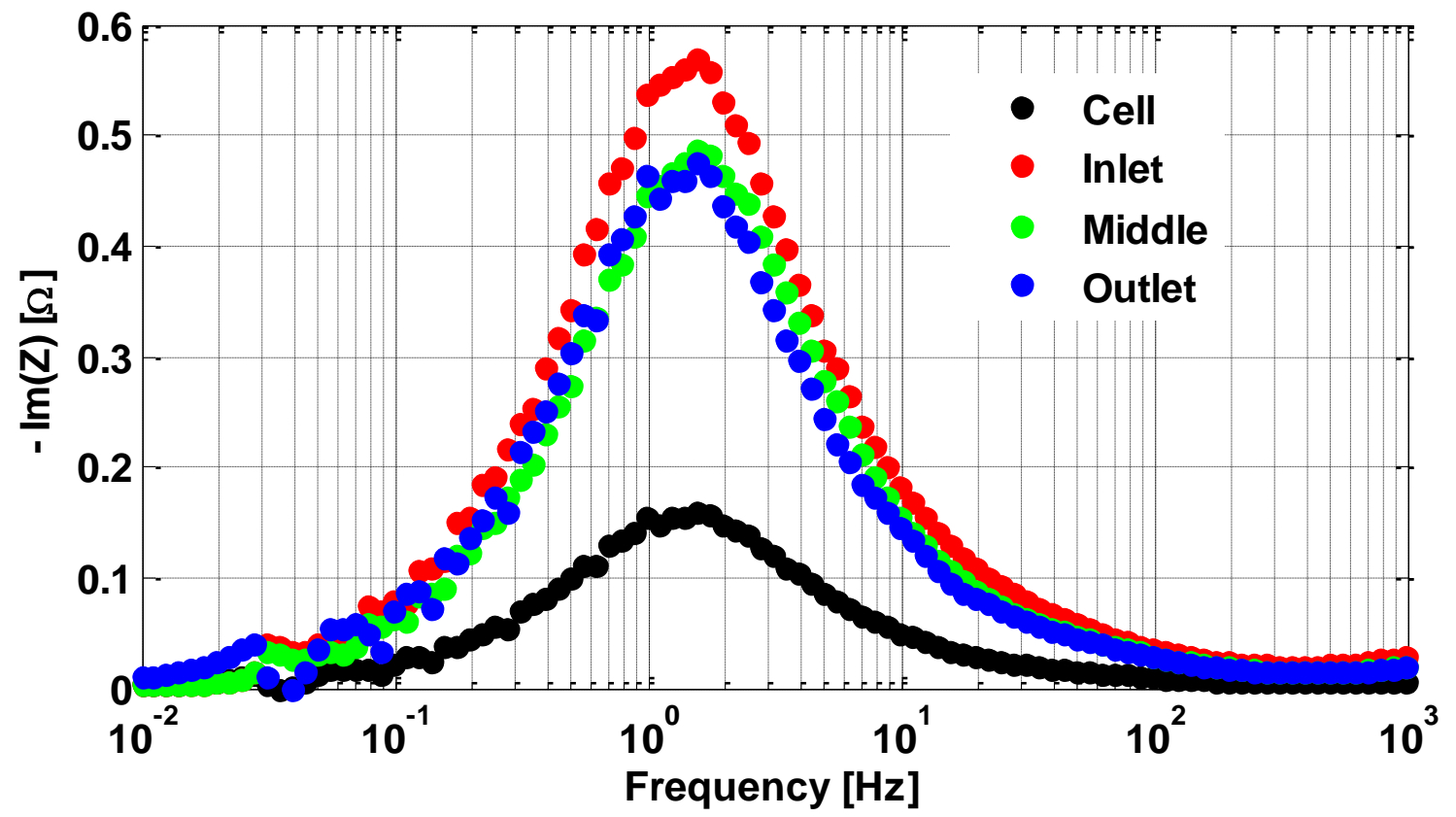

Figure 5.23: Predicted impedance curves for planar cell in different regions (inlet, middle and outlet) at $4 \mathrm{~h}$ of phosphine exposure: Nyquist representation (top) and bode representation (bottom) 

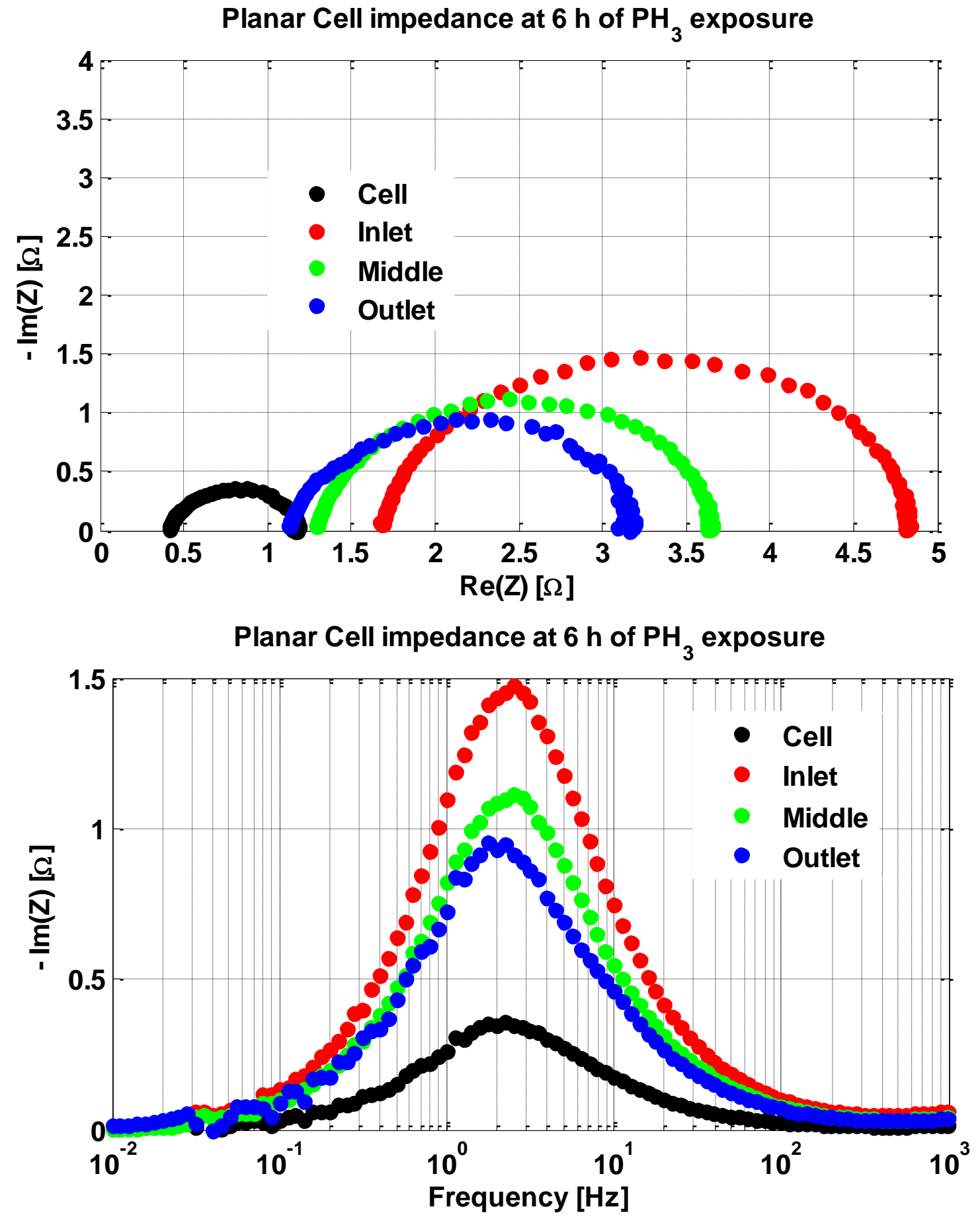

Figure 5.24: Predicted impedance curves for planar cell in different regions (inlet, middle and outlet) at $6 \mathrm{~h}$ of phosphine exposure: Nyquist representation (top) and bode representation (bottom) 

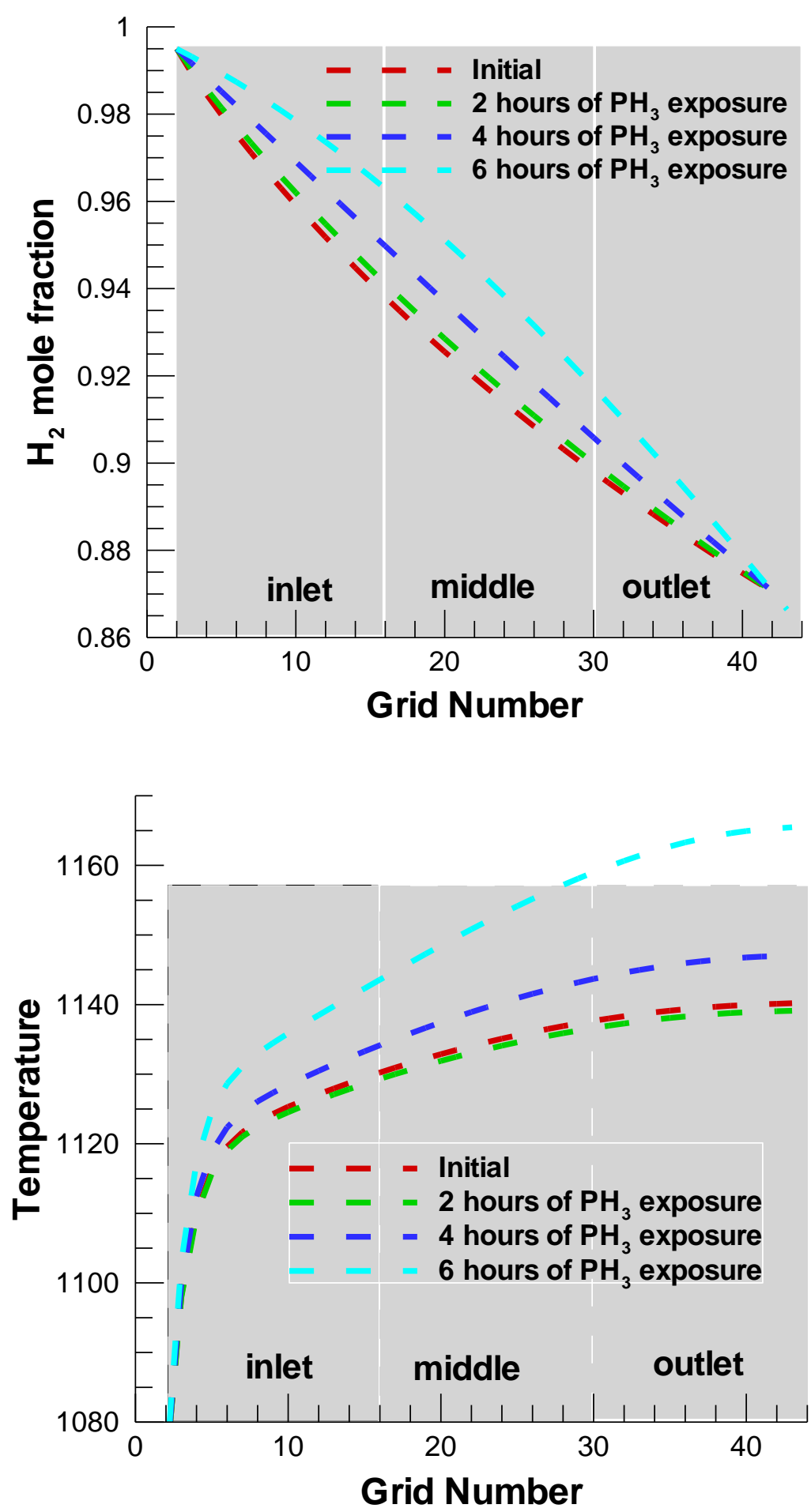

Figure 5.25: Hydrogen mole fraction (top) and Temperature (bottom) variation along the fuel channel at different times of phosphine exposure 


\subsection{Conclusions}

An existing phenomenological model for contaminant induced SOFC anode degradation is incorporated into a three dimensional SOFC modeling code. A representative repeat unit of a large planar cell is chosen as the computational domain and simulations are performed for a cell operating at $0.1 \mathrm{~A} / \mathrm{cm}^{2}$ and exposed to $10 \mathrm{ppm}$ of phosphine in the fuel channel. Results show that after some initial transients, there is a rapid degradation until circa $35 \%$ performance loss after which spurious oscillations are observed in the simulations. Normally, a $50 \%$ performance loss can be considered as total cell failure. Current redistribution caused by contaminant induced deactivation of some regions of the cell is shown to result in hydrogen starvation in the active regions which in turn leads to the oscillations in the voltage and the current. The flexibility of planar cells being able to redistribute the electrochemical activity is in stark contrast to what is usually observed in button cells where all the distributions are essentially one dimensional. Additional simulations are performed to diagnose the electrochemical behavior of the cell at different times of the gradual performance loss, by simulating the V-I behavior and the impedance response of the cell using the current states of gradual anode degradation. The results show that the initial changes in V-I curves with exposure time were mostly due to increase in activation losses. These observations are approximately consistent with the impedance analyses. In addition, a comparison between button and planar cell was presented. The current redistribution and the temperature variation in planar cell simulation are the key physical behaviors that make the planar cell perform better than the button cell. 


\section{CHAPTER 6 - Conclusions and future work}

\subsection{Conclusions}

A one-dimensional (1D) performance degradation model is used to investigate the $\mathrm{PH}_{3}$ poisoning in the anode of SOFCs under different steam concentrations by examining the transport and reactions both in the gas phase and in the solid/surface phase. The model parameters are calibrated using the experiments conducted at NIFT (National Institute of Fuel-Cell Technology), WVU as a guide to simulate the effect of steam concentration. The calibrated model was used to estimate performance degradation for both wet and dry fuel case with $10 \mathrm{ppm} \mathrm{PH}_{3}$. A supplementary computational model was integrated into the degradation model to evaluate performance of the cell at different times of the gradual performance loss, by simulating the V-I behavior and the impedance response of the cell using the instantaneous states of gradual anode degradation process.

Using the 1D degradation model, a sensitivity analysis was performed to investigate the influence of empirical model parameters on model outputs, electrical potential, ohmic and polarization losses. Both dual number automatic differentiation (DNAD) and finite difference (FD) method were used in this analysis. Good agreement between DNAD and FD methods builds confidence the present analysis. The sensitivities obtained from DNAD could be different from the exact derivatives of the unknown true solution to the mathematical model due to inherent errors in numerical solution, such as discretization errors. The sensitivity to most parameters increased as the cell degradation increases. 
For further validation of the degradation model, experimentally observed elemental redistribution of anode supported SOFC due to arsine exposure is simulated by using a multi-dimensional inhouse code (DREAM-SOFC) designed for simulations of large planar SOFCs. Experimentally observed sharp boundaries for the secondary phases formation are obtained by simulations for the trace element coverage. Simulations and experimental analysis indicate that, for fuels contain low contaminant concentrations $(\leq 1 \mathrm{ppm})$, degradation can still occur, albeit at much more reduced degradation rates.

A multi-dimensional code (DREAM-SOFC) designed for simulation of large scale planar solid oxide fuel cells is extended by integrating the contaminant degradation model to predict the degradation patterns observed in SOFCs when anode is exposed to phosphine. A representative repeat unit of a large planar cell is chosen as the computational domain and simulations are performed for a cell operating at $0.1 \mathrm{~A} / \mathrm{cm}^{2}$ and exposed to $10 \mathrm{ppm}$ of phosphine in the fuel channel. It is found that there is a steady degradation until approximately $35 \%$ performance loss after which spurious oscillations are observed in the simulations. Normally a $50 \%$ performance loss can be considered as total cell failure. Current redistribution caused by contaminant induced deactivation of some regions of the cell is shown to result in hydrogen starvation in the active regions which in turn leads to instabilities in the calculations. The flexibility of planar cells being able to redistribute the electrochemical activity is in stark contrast to what is usually observed in button cells where all the distributions are essentially one dimensional. The electrochemical characteristic of the phosphine induced planar cell degradation is diagnosed by performing additional simulations for V-I behavior and impedance response at different degradation levels under continuous $\mathrm{PH}_{3}$ exposure. 
In addition a transport model for nickel migration is formulated based on mechanisms such as electro-migration, diffusion and formation of secondary phases which are believed to be responsible for Ni migration and it is integrated with SOFC anode contaminant degradation model. This model is capable of predicting experimentally observed Ni-Migration patterns.

\subsection{Future work}

- Sensitivity analysis using DNAD approach can be implemented into DREAM-SOFC directly.

- The run time of DREAM-SOFC code, should be accelerated to simulate SOFC stacks.

- Mechanical stresses due to temperature variation can be incorporated into DREAM-SOFC

- Sinusoidal voltage excitation technique for the impedance analysis should be implemented into 3D planar cell code to assess the accuracy of the rapid impedance technique.

- The one-dimensional performance degradation model can be extended to include the detailed kinetics of formation for secondary phases inside the anode.

- Impedance and polarization analysis for the 10 channel planar cell 


\section{REFERENCES}

[1] Roper, L. D. (2014, Feb.) Genealogy Web Page of L. David Roper. [Online]. http://www.roperld.com/science/energyfuture.htm

[2] W. C. Association. (2014, Feb.) World Coal Org. [Online]. http://www.worldcoal.org/coal/where-is-coal-found/

[3] Tverberg, G. (2014, Feb.) The Oil Drum. [Online]. http://www.theoildrum.com/node/9452

[4] J.P. Trembly, R.S. Gemmen, and D.J. Bayless, "The effect of coal syngas containing AsH3 on the performance of SOFCs: Investigations into the effect of operational temperature, current density and AsH3 concentration," Journal of Power Sources, vol. 171, pp. 818825, 2007.

[5] J. Zhang, H. Wang, D.P. Wilkinson, D. Song, J. Shen, and Z. Liu, "Model for the contamination of fuel cell anode catalyst in the presence of fuel stream impurities," Journal of Power Sources, vol. 147, pp. 58-71, 2005.

[6] Cayan, N., "A degradation model for solid oxide fuel cell anodes due to impurities in coal syngas," PhD Thesis, West Virginia University, Morgantown, WV, USA, 2010.

[7] J.B. Hansen, "Correlating sulfur poisoning of SOFC nickel anodes by a Temkin isotherm," Electrochemical and Solid-State Letters," vol. 11, pp. B178-B180, 2008.

[8] J. Bao, G.N. Krishnan, P. Jayaweera, J. Perez-Mariano, and A. Sanjurjo, "Effect of various coal contaminants on the performance of solid oxide fuel cells: Part I. Accelerated testing," Journal of Power Sources, vol. 193, pp. 607-616, 2009. 
[9] A.I. Marquez, T.R. Ohrn, J.P. Trembly, D.C. Ingram, and D.J. Bayless, "Effects of coal syngas and H2S on the performance of solid oxide fuel cells Par 2. Stack tests," Journal of Power Sources, vol. 164, pp. 659-667, 2007.

[10] Y. Matsuzaki and I. Yasuda, "The poisoning effect of sulfur-containing impurity gas on a SOFC anode: Part I. Dependence on temperature, time and impurity concentration," Solid State Ionics, vol. 132, pp. 261-269, 2000.

[11] DoITPoMS. (2006) DoITPoMS, University of Cambridge. [Online]. http://www.doitpoms.ac.uk/tlplib/fuel-cells/sofc_electrolyte.php?printable=1

[12] Cayan, F. N., Pakalapati, S. R., Celik, I., Xu, C., Zondlo, J., "A Degradation Model for Solid Oxide Fuel Cell Anodes due to Impurities in Coal Syngas: Part I Theory and Validation," Fuel Cells, vol. 12, no. 3, p. 464-473, 2011.

[13] Zittel, W., Bölkow, L., Schindler, J., "Energy Watch Group Coal: Resources and Future Production," July, 2007.

[14] Fossil Energy Office of Communications. (2011, Feb.) U. S. Department of Energy. [Online]. http://www.fossil.energy.gov/programs/powersystems/fuelcells/fuelcells_solidoxide.html

[15] Chen, Y., Chen, S., Hackett, G., Finklea, H., Zondlo, J., Celik, I., Song, X., Gerdes, Kirk,G., "Microstructure degradation of YSZ in Ni/YSZ anodes of SOFC operated in phosphine-containing fuels," Solid State Ionics, pp. 25-32, 2013.

[16] Cayan, F. N., Zhi, M., Pakalapati, S. R., Celik, I., Wu, N. Q., Gemmen, R., "Effects of coal syngas impurities on anodes of solid oxide fuel cells," Journal of Power Sources, vol. 185, p. 595-602, 2008.

[17] Haga, K., Shiratori, Y., Nojiri, Y., Ito, K. and Sasaki, K., "Phosphorus Poisoning of NiCermet Anodes in Solid Oxide Fuel Cells," Journal of the Electrochemical Society, vol. 157, no. 11, pp. 1693-1700, 2010.

[18] Marina O.A., Coyle C.A., Thomsen E.C., Edwards D.J., CoffeyG.W., PedersonL.R., "Degradation mechanisms of SOFC anodes in coal gas containing phosphorus," Solid State Ionics, 181, pp. 430-440, 2010. 
[19] Guo, H., Iqbal, G. and Kang, B., "Investigation of secondary phases formation due to ph3 interaction with sofc anode," International Journal of Applied Ceramic Technology, vol. 8, no. 1, pp. 68-73, 2011.

[20] Celik I. B., Wu, N., Finklea, H., Kang, B., Liu, X., Zondlo, J., Song, X., Simirnow, A., Bajura R. A., "Utilization of Coal Syngas in High Temperature Fuel Cells: Degradation Mechanisms and Lifetime Prediction," Office of Fossil Energy Fuel Cell Program III. B. 2, Morgantown, Annual Report, 2012.

[21] Zhi, M., Cayan, F. N., Celik, I., Gemmen, R., Pakalapati, S. R., Wu, N. Q., "Temperature and Impurity Concentration Effects on Degradation of Nickel/Yttria-stabilized Zirconia Anode in PH3-Containing Coal Syngas," Fuel Cell, vol. 10, no. 1, pp. 174-180, 2010.

[22] Heuer, J. K., Okamoto, P. R., Lam, N. Q., Stubbins, J. F., "Relationship between segregation-induced intergranular fracture and melting in nickel sulfur system," Applied Physics Letter, 76, 23, 2000.

[23] Kishimoto, H., Yamaji, K., Brito, M. E., Horita, T. and Yokokawa, H., "Generalized Ellingham Diagrams for Utilization in Solid Oxide Fuel Cells," Journal of Mining and Metallurgy 44 B, pp. 39-48, 2008.

[24] Kengo Haga, Yusuke Shiratori, Kohei Ito, Kazunari Sasaki, "Chemical Degradation and Poisoning Mechanism of Cermet Anodes in Solid Oxide Fuel Cells," in 216th ECS Meeting, , Vienna, 2009, p. Abstract\#1333.

[25] Blancas, F. E., Pakalapai, S. R., Escobar, J. A., Celik, I. B., "Numerical evaluation and comparison of different reduced mechanisms for predicting the performance of a sofc operating on coal syngas," in ASME Fluids Engineering Division Summer Conference, Jacksonville, Florida, USA, 2008.

[26] Zhu, H., Kee, R. J., Janardhanan, V. M., Deutschmann, O., Goodwinc, D. G., "Modeling Elementary Heterogeneous Chemistry," Journal of The Electrochemical Society, 152, pp. A2427-A2440, 2005.

[27] Zhu, H., Kee, R. J., "Modeling Distributed Charge-Transfer Processes in SOFC," Journal of The Electrochemical Society, 155, pp. B715-B729, 2008. 
[28] Stefan, G., Wolfgang G. Bessler, W. G., "Physically based impedance modeling of Ni/YSZ cermet anodes," Journal of The Electrochemical Society, vol. 155, no. 9, pp. B937-B952, 2008.

[29] Finklea, H., Chen, X., Gerdes, K., Pakalapati, S., and Celik, I. B., "Analysis of SOFCs using reference electrodes,"Journal of Electrochemical Soc., 160(9) , F1055-F1066, 2013.

[30] L. L. Green, P. A. Newman, and K. J. Haigler, "Sensitivity derivatives for advanced CFD algorithm and viscous modeling parameters via automatic differentiation," Journal of Computational Physics, vol. 125, no. 2, pp. 313-324, 1996.

[31] C. H. Bischof, H. M. Bucker, and A. Rasch, "Sensitivity analysis of turbulence models using automatic differentiation," SIAM Journal on Scientific Computing, vol. 26, no. 2, pp. 510-522, 2004.

[32] N. J. Brown and K. L. Revzan, "Comparative sensitivity analysis of transport properties and reaction rate coefficients," International journal of chemical kinetics, vol. 37, no. 9, pp. 538-553, 2005.

[33] C. H. Bischof, H. M. Bucker, A. Rasch, E. Slusanschi, and B. Lang, "Automatic differentiation of the general-purpose computational fluid dynamics package FLUENT," Journal of fluids engineering, vol. 129, no. 5, pp. 652-658, 2007.

[34] Bardow, A.; Bischof, C.H.; Bucker, H. Martin; Dietze, G.; Kneer, R.; Leefken, A.; Marquardt, W.; Renz, U.; Slusanschi, E., "Sensitivity-based analysis of the k-epsilon model for the turbulent flow between two plates," Chemical Engineering Science, vol. 63, no. 19, pp. 4763-4775, 2008.

[35] Alexe, M.; Roderick, O.; Utke, J.; Anitescu, M.; Hovland, P.; Fanning, T.; others, "Automatic differentiation of codes in nuclear engineering applications," Technical report, Mathematics and Computer Science Division. Argonne National Lab, Tech. rep., 2009.

[36] A. Carle, L. Green, P. Newman, and C. Bischof, "Applications of automatic differentiation in CFD," Argonne National Lab., IL (United States), Tech. rep., 1994. 
[37] Kioutsioukis, Ioannis; Melas, Dimitrios; Zerefos, Christos; Ziomas, Ioannis, "Efficient sensitivity computations in 3D air quality models," Computer Physics Communications, vol. 167, no. 1, pp. 23-33, 2005.

[38] Tomlin, A.S., "The role of sensitivity and uncertainty analysis in combustion modelling," Proceedings of the Combustion Institute, 2012.

[39] A. Griewank and A. Walther, "Evaluating derivatives: principles and techniques of algorithmic differentiation". Society for Industrial and Applied Mathematics (SIAM), 2008.

[40] C. Bischof, P. Khademi, G. Pusch, and A. Carle, "Automatic differentiation: Obtaining fast and reliable derivatives--fast," Argonne National Lab., IL (United States), Tech. rep., 1994.

[41] Stamatiadis, S.; Prosmiti, R.; Farantos, SC, "AUTO\_DERIV: Tool for automatic differentiation of a FORTRAN code," Computer Physics Communications, vol. 127, no. 23, p. 343, 2000.

[42] Sherman, L.L.; III, A.C. Taylor; Green, L.L.; Newman, P.A.; Hou, G.W.; Korivi, V.M., "First-and second-order aerodynamic sensitivity derivatives via automatic differentiation with incremental iterative methods," Journal of Computational Physics, vol. 129, no. 2, pp. 307-331, 1996.

[43] Hwang, D.; Byun, D.W.; Odman, M. Talat, "An automatic differentiation technique for sensitivity analysis of numerical advection schemes in air quality models," Atmospheric Environment, vol. 31, no. 6, pp. 879-888, 1997.

[44] G. R. Carmichael, A. Sandu, and others, "Sensitivity analysis for atmospheric chemistry models via automatic differentiation," Atmospheric Environment, vol. 31, no. 3, pp. 475489, 1997.

[45] Spall, R.E.; Yu, W., "Imbedded Dual-Number Automatic Differentiation for Computational Fluid Dynamics Sensitivity Analysis," Journal of Fluids Engineering, vol. 135, p. 014501, 2013. 
[46] Sezer, H., Pakalapati, S. R., Celik, I., "The effect of steam concentration on phosphine induced sofc anode degradation: a computational study," in 5th International Conference on Fundamentals \& Development of Fuel Cells, Karlsruhe, 2013.

[47] Elizalde-Blancas, F., Celik, I. B.,Rangel-Hernandez, V., Hernandez-Guerrero, A., RiescoAvila, J., "Numerical modeling of SOFCs operating on biogas from biodigesters,"

International Journal of Hydrogen Energy, no. 38, pp. 377-384, 2013.

[48] R., Pakalapati S., "A new reduced order model for solid oxide fuel cells," PhD Thesis, West Virginia University, Morgantown, 2006.

[49] Mahcene, H., Moussa, H. B., Bouguettaia, H., Bechki, D., Babay, S., Meftah, M. S., "Study of species, temperature distributions and the solid oxide fuel cells performance in a 2-D model," International Journal of Hydrogen Energy, no. 36, pp. 4244-4252, 2011.

[50] Recknagle, K. P., Ryan, E. M., Koeppel, B. J., Mahoney, L. A., Khaleel, M. A., "Modeling of electrochemistry and steam-methane reforming performance for simulating pressurized solid oxide fuel cell stacks," Journal of Power Sources, no. 195, pp. 6637-6644, 2010.

[51] Huang, C. M., Shy, S. S., Lee, C. H., "On flow uniformity in various interconnects and its influence to cell performance of planar SOFC," Journal of Power Sources, no. 183, pp. 205-213, 2008.

[52] Qu, Z., Aravind, P. V., Boksteen, S. Z., Dekker, N. J. J., Janssen, A. H. H., Woudstra, N., Verkooijen, A. H. M., "Three-dimensional computational fluid dynamics modeling of anode-supported planar SOFC," International Journal of Hydrgoen Energy, no. 36, pp. 10209-10220, 2011.

[53] Elizalde-Blancas, F., "Modeling Issues for Solid Oxide Fuel Cells Operating with Coal Syngas," PhD Thesis, West Virginia University, Morgantown, 2009.

[54] Celik, I. B. and Pakalapati S. R., "A modular Approach to SOFC Modeling," OAK Ridge Associated Universities (ORAU), OAK Ridge, TN, Research report, 2003.

[55] Pakalpati, S. R., "Numerical Study of Current Distribution Inside the Cathode and Electrolyte on a Solid Oxide Fuel Cell," M.Sc. Thesis, Mechanical and Aerospace Engineering Department, West Virginia University, Morgantown, West Virginia, 2003. 
[56] Pakalapatia, S., Kirk Gerdes, K., Finkleaa, H., Gonga, M., Liua, X., Celik, I. B. , "Micro scale dynamic modeling of LSM/YSZ composite cathodes," Solid State Ionic, vol. 258, p. 45-60, May 2014.

[57] Bessler, W. G., "Gas concentration impedance of solid oxide fuel cell anodes I. Stagnation point flow geometry," Journal of The Electrochemical Society, vol. 153, no. 8, pp. A1492A1504, 2006.

[58] T. Yang; I. B. Celik; S. R. Pakalapati; H. O. Finklea; K. Gerdes, "Validation of Various Oxidation and Reduction Models for SOFC Electrodes," in 225th ECS Meeting May 11-16, Orlando, FL, 2014.

[59] Pakalapati, R., Sezer, H., Celik, I., "IMPLICATIONS OF USING DUAL NUMBER DERIVATIVES WITH A NUMERICAL SOLUTION," in Proceedings of the ASME 2013 Fluids Engineering Summer Meeting FEDSM2013, Incline Village, Nevada, USA, 2013.

[60] S. Patankar, Numerical heat transfer and fluid flow. Taylor $\ \&$ Francis, 1980.

[61] Taylor, C R N. [Online]. https://gist.github.com/2005955

[62] Sezer, H., Pakalapati, S. R., Celik, I., "Phosphine Induced Anode Performance Degradation in a Planar SOFC: A Numerical Study," ECS Trans., vol. 57, no. 1, pp. 2679-2689, 2013.

[63] J.W. Stevenson, O.A. Marina, C.A. Coyle, and D.J. Edwards, "Effects of Phosphorus and Arsenic on SOFC Anodes," 12 th Annual SECA Workshop, 2011.

[64] Wolfgang G. Bessler, "Rapid Impedance Modeling via Potential Step and Current Relaxation Simulations," Journal of The Electrochemical Society, vol. 154, no. 11, pp. 1186-1191, 2007.

[65] Lussier, A., Sofie, S., Duorak, J., Idzerda, Y. U., "Mechanism for SOFC anode degradation from hydrogen sulrfure exposure," International Journal of Hydrogen Energy, vol. 33, pp. 3945-3951, 2008.

[66] Demircan, O., Zhang, W., Xu, C., Zondlo, J., Finklea, H. O., "The effect of over potential on performance degradation of the solid oxide fuel cell Ni/YSZ anode during exposure to 
syngas with phosphine contaminant," Journal of Power Sources, vol. 195, pp. 3091-3096, 2010 .

[67] Xu, C., Zondlo, J. W., Finklea, H. O., Demircan, O., Gong, M., Liu, X., "The effect of phosphine in syngas on Ni-YSZ anode-supported solid oxide fuel cells," Journal of Power Sources, vol. 193, pp. 739-746, 2009.

[68] Zhi, M., Chen, X., Finklea, H., Celik, I., Wu, N. Q., "Electrochemical and microstructural analysis of nickel-yttria-stabilized zirconia electrode operated in phosphorus-containing syngas," Journal of Power Sources, vol. 183, no. 2, pp. 485-490, Sep. 2008.

[69] Silva, Kandaudage Channa R. De, "A High Temperature Planar Solid Oxide Fuel Cell Operating on Phosphine," PhD Thesis, the Russ College of Engineering and Technology of Ohio University, 2011.

[70] Krumbein, S. J., "Metallic Electromigration Phenomena," in 33rd Holm Conference on Electrical Contacts, Chicago, Illinois, 1987.

[71] Yost, D.E., "Silver Migration in Printed Circuit," in in Proc. Symp. on Printed Circuits, Philadelphia, 1955.

[72] Kohman, G. T., Hermance, H. W., Downes, G. H., "Silver Migration in Electrical Insulation," Bell Syst. Tech. J., vol. 34, p. 1115, 1955.

[73] Jenson, K.V., Wallenberg, R., Chorkendorff, I., Mogensen, M., "Effect of impurities on structural and electrochemical properties of the Ni-YSZ interface," Solid State Ionics, vol. 160, pp. 27-37, 2003.

[74] Popovich, M., Mezhiba, A. V., Friedman, E. G., "Electromigration," in Power Distribution Networks with On-Chip Decoupling Capacitors. New York: Springer Science, 2008, ch. 4, pp. 71-86.

[75] Brophy, J. H., Rose, R. M., and Wulff, J., The Structure and Properties of Materials. New York, USA: John Wiley \& Sons, Inc, 1964, vol. Thermodynamics of Structure.

[76] Tu, K. N., "Recent advances on electromigration in very-large-scale-integration of interconnects," Journal of Applied Physics, vol. 94, no. 7, pp. 5451-5473, Nov. 2003. 
[77] Pierce, D. G., Brusius, P. G., "ELECTROMIGRATION: A REVIEW," Microelectron. Reliab., vol. 37, no. 7, pp. 1053-1072, 1997.

[78] Zhang, J. Electromigration and Related Integrated Circuit (IC) Failure. Materials Engineering Dept. Drexel University, PA. Jul. 2001.

[79] Otsuka, K., Kawanobe, T., "Metal Migration in Electronic Components," in in Proc. Electronic Components Conf., 1982, p. 220.

[80] Steppan, J. J., Roth, J. A., Hall, L. C., Jeannotte, D. A., and Carbone, S. P., "A Review of Corosion Failure Mechanisms During Accelerated Tests: Electrolytic Metal Migration," $J$. Electrochem. Soc., vol. 134, p. 175, 1987.

[81] Krumbein, S. J., and Reed, A. H., "New Studies of Silver Electromigration," in Proc. 9th ht. Conf. on Electric Contact Phenomena, 1978, p. 145.

[82] DiGiacomo, G., "Metal Migration ( $\mathrm{Ag}, \mathrm{Cu}, \mathrm{Pb}$ ) in Encapsulated Modules and Time-ToFail Model as a function of the Environment and Package Properties," in in Proc. ht. Reliability Physics Symp., 1982, p. 27.

[83] Buerke, A., Wendrock, H., Wetzig, K., "Study of Electromigration Damage in Al Interconnect," Cryst. Res. Technol., vol. 35, p. 721-730, 2000.

[84] Krumbein, S., J., "Tutorial: Electrolytic Models for Metallic Electromigration Failure Mechanisms," IEEE Transactions on Reliability, vol. 44, no. 4, Dec. 1995.

[85] Finklea, H., "Diffusion mechanism in SOFC anode under operating condition," West Virginia University ,Private communication, Aug. 2014. 


\section{ApPendix A}

\section{A.1 Comparison of planar cell and button cell}

Simulations are performed with the same calibrated parameters and similar operating conditions that are listed in Table 5.2 for button and planar cells. As it is depicted in Figure A.1, there is a significant difference between button cell and planar cell performance. Planar cell exposed to phosphine performs better than the button cell. This behavior could be explained by the current redistribution and the temperature increase in the planar cell. As it was earlier indicated that the phosphine coverage propagation along the anode yields the current redistribution inside the planar cell, however this type of behavior is not possible to occur in button cell simulations. This is just due to the geometric orientation of the button cells. It also should be noted that, the energy equation is not solved for the button cell degradation simulations. Therefore the temperature is kept constant

during the phosphine exposure. Constant temperature does not yield any variation in the cell polarization and ohmic resistance. 

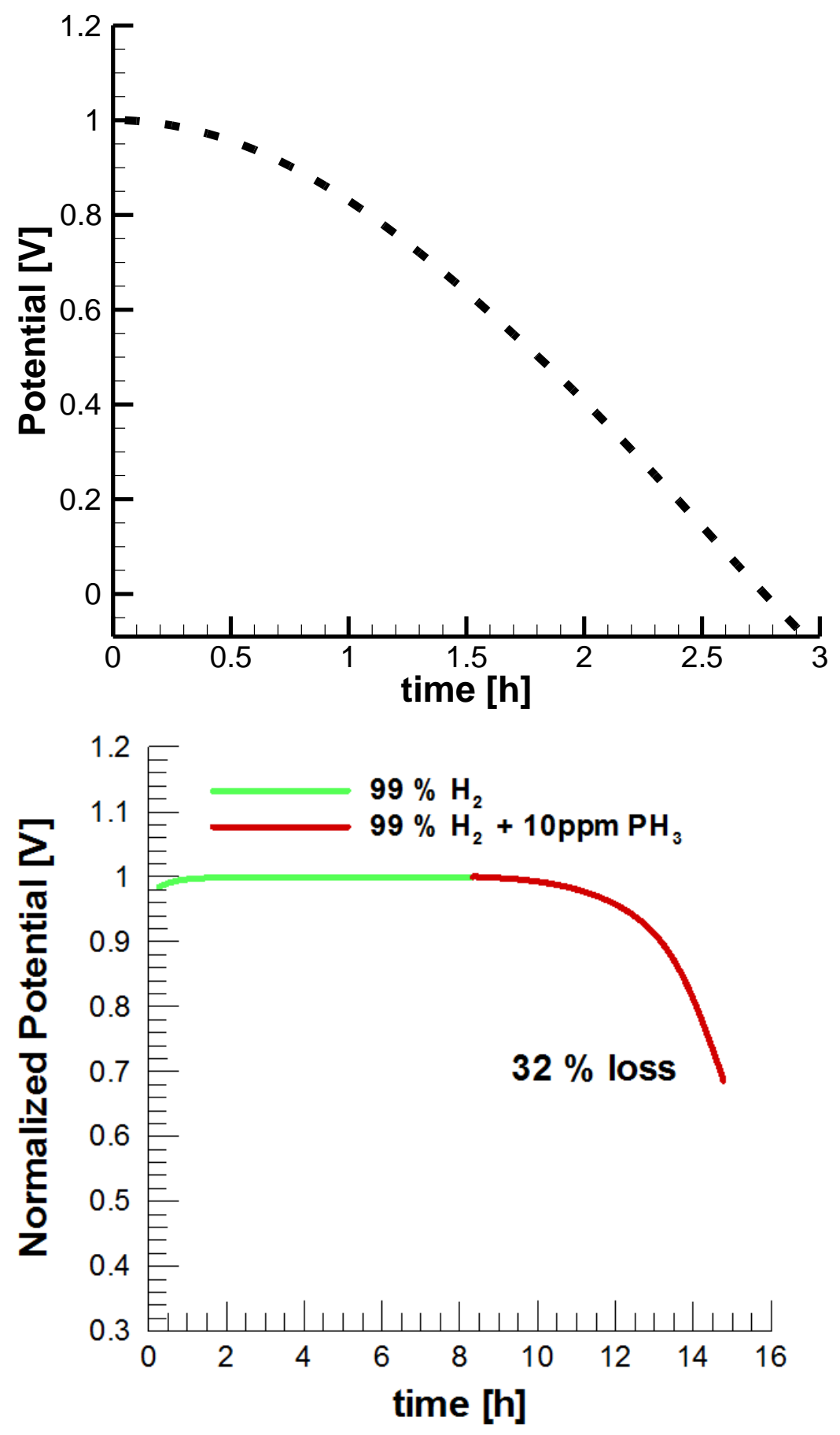

Figure A.1: Predicted cell voltage under $10 \mathrm{ppm} \mathrm{PH}_{3}$ exposure: button cell (top), planar cell (bottom) 


\section{A.2 Additional V-I curves for different part of the cell}
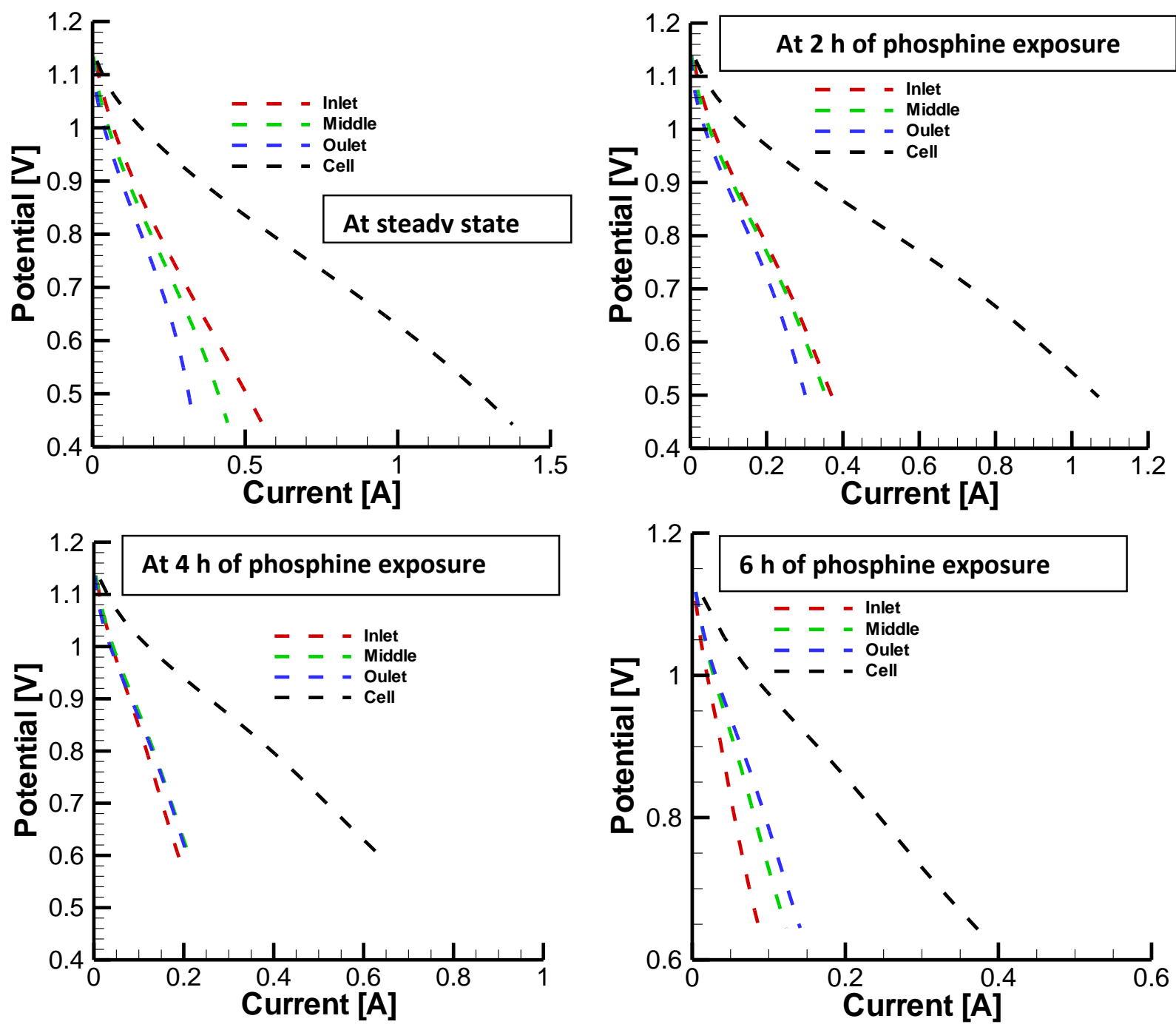

Figure A.2: Predicted V-I curves of planar cell at different times of phosphine exposure for dry hydrogen in different sections of the cell along the channel. 


\section{A.3 Simulated time-traces of exciting current and resulting potential relaxation plots}
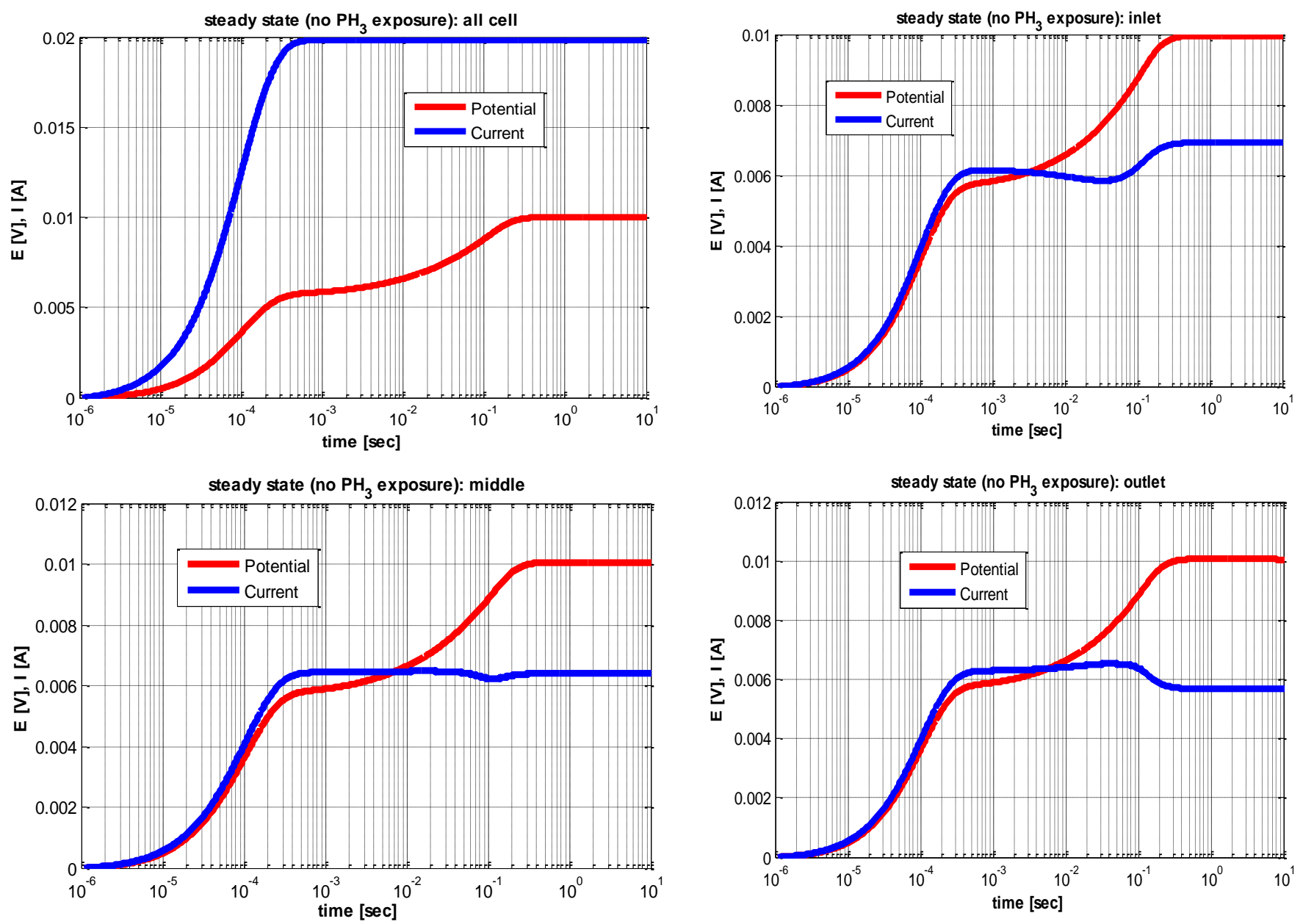

Figure A.3: Time trace of exciting current and potential relaxation (exponential step $\boldsymbol{\tau}=\mathbf{1 0}^{\mathbf{- 4}}$ ) for impedance analysis of planar cell simulated by DREAM-SOFC in different regions (inlet, middle and outlet) at steady state condition of the cell 

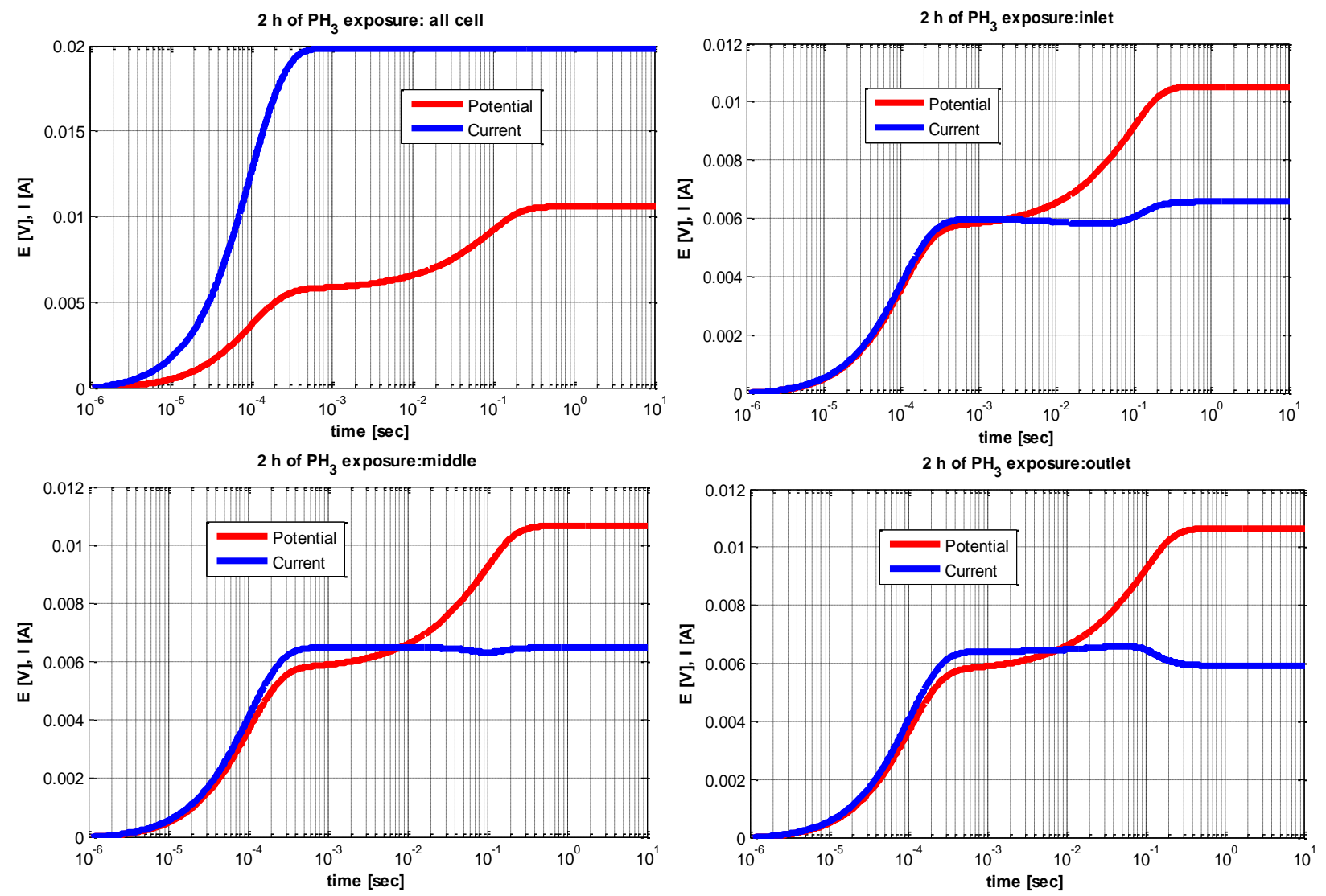

Figure A.4: Time trace of exciting current and potential relaxation (exponential step $\boldsymbol{\tau}=$

$\mathbf{1 0}^{-4}$ ) for impedance analysis of planar cell simulated by DREAM-SOFC in different regions

(inlet, middle and outlet) at $2 \mathrm{~h}$ of phosphine exposure 

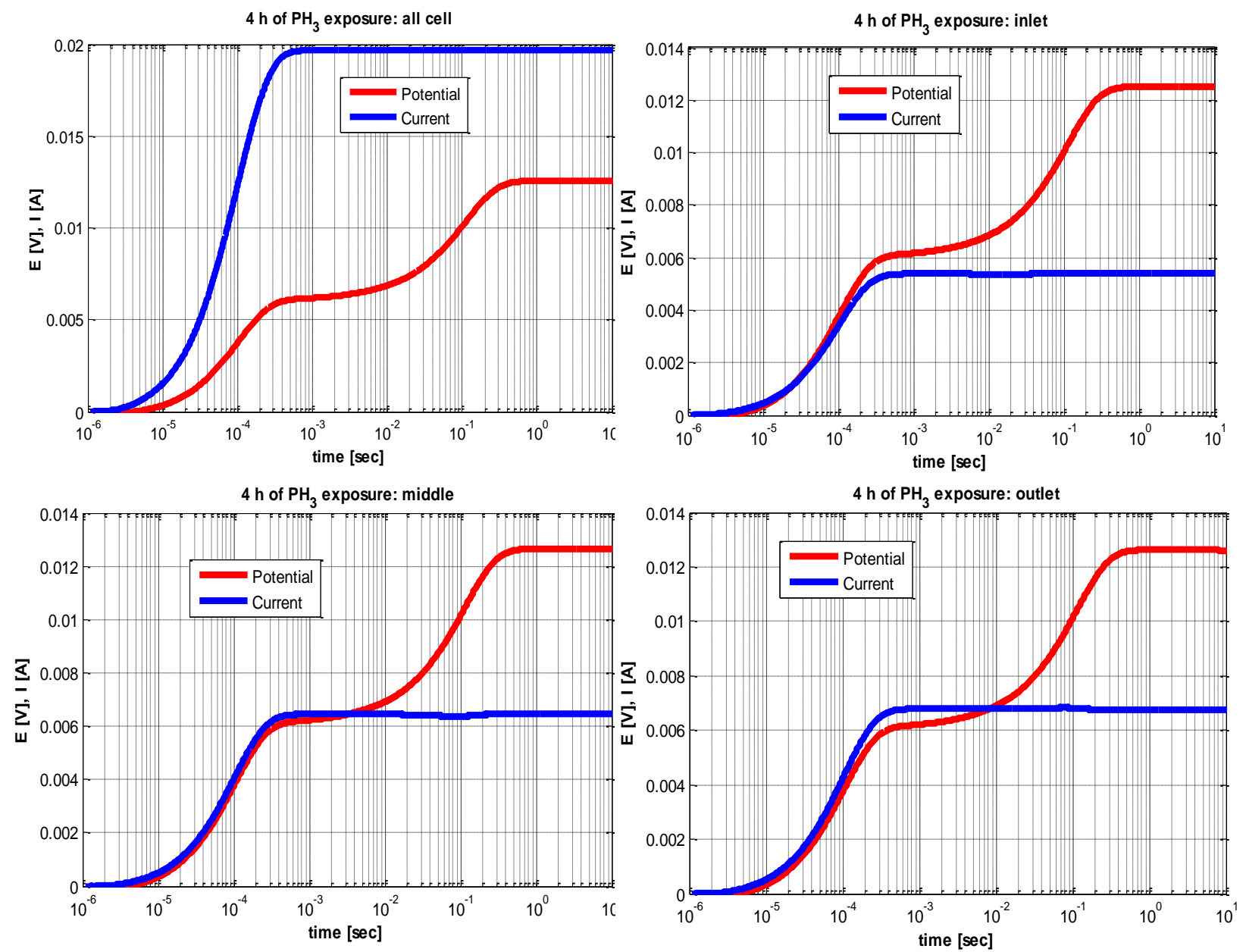

Figure A.5: Time trace of exciting current and potential relaxation (exponential step $\boldsymbol{\tau}=$

$\mathbf{1 0}^{-4}$ ) for impedance analysis of planar cell simulated by DREAM-SOFC in different regions

(inlet, middle and outlet) at $4 \mathrm{~h}$ of phosphine exposure 

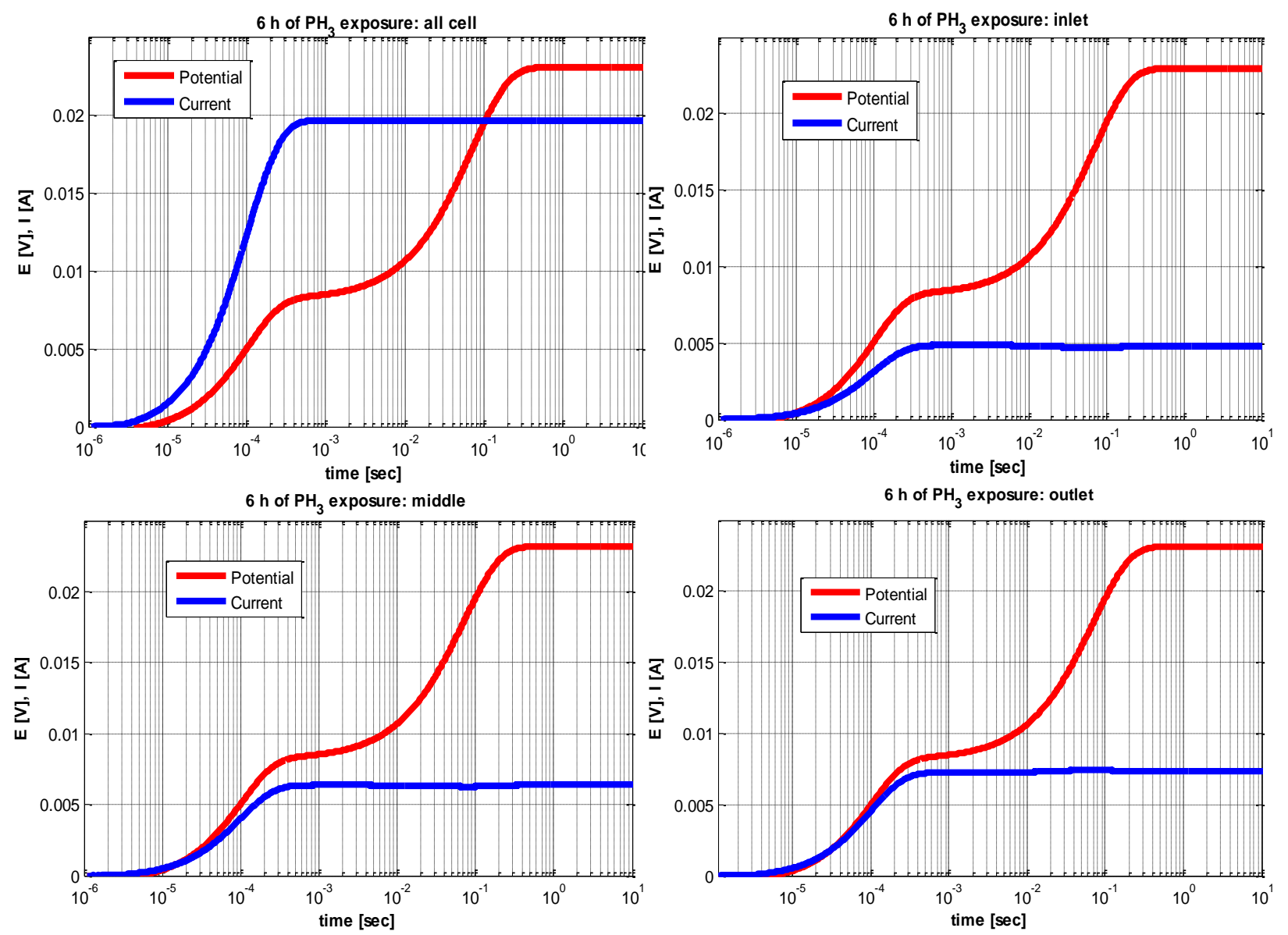

Figure A.6: Time trace of exciting current and potential relaxation (exponential step $\boldsymbol{\tau}=$ $\mathbf{1 0}^{-4}$ ) for impedance analysis of planar cell simulated by DREAM-SOFC in different regions (inlet, middle and outlet) at $6 \mathrm{~h}$ of phosphine exposure 


\section{A.4 Over all cell impedance}

A comparison between simulation and experimental results at steady state without phosphine exposure is shown in Figure A.7. The experiment was performed under $1 \mathrm{~A}$ of total current load. The corresponding current density is $0.031 \mathrm{~A} / \mathrm{cm}^{2}$. The impedance calculations for the planar cell are simulated under $0.1 \mathrm{~A} / \mathrm{cm}^{2}$. As it can be observed in the Figure A.7, the most significant difference occurs in the region of low frequencies. This difference is due to the different current conditions. However, similar results are obtained with the experimental data for ohmic resistance. Overall, as it can be seen in Figure A.7, a good agreement is achieved between model and experimental data.

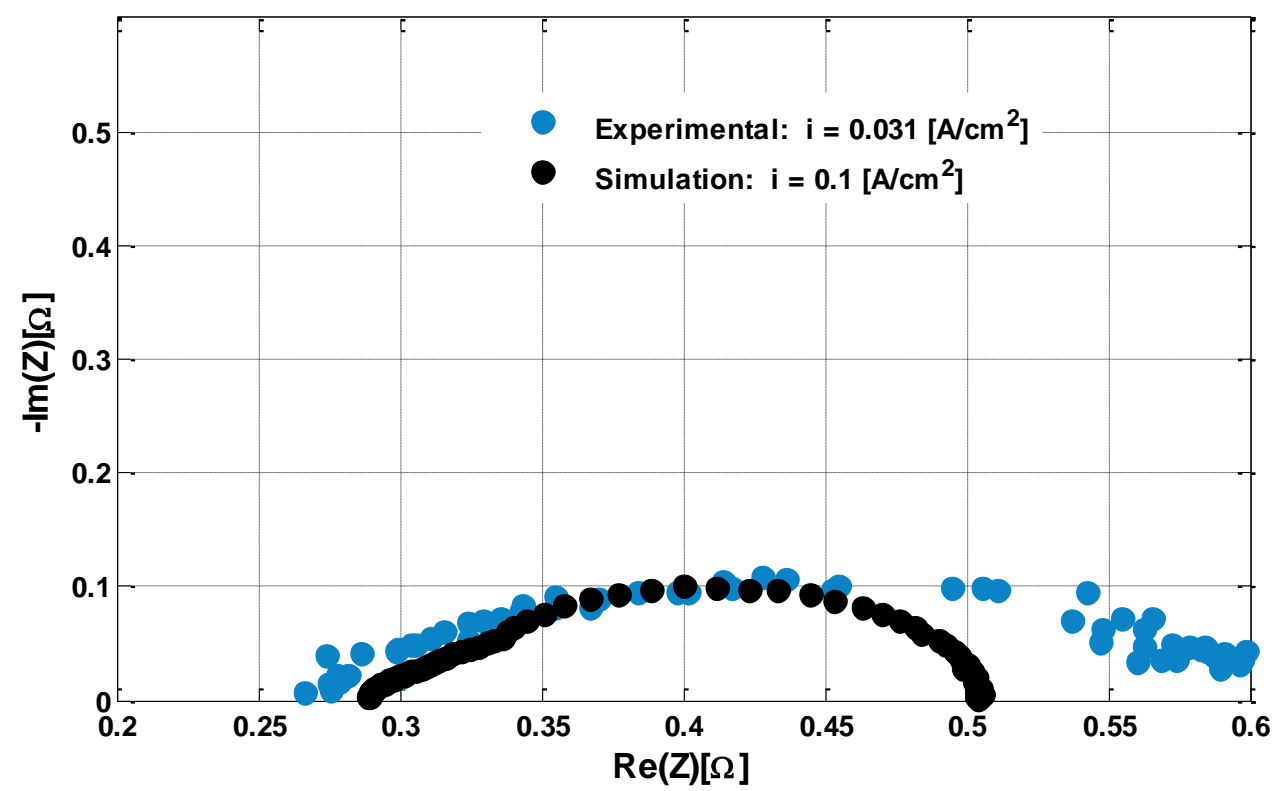

Figure A.7: Comparison of simulation and experiment at steady state condition 

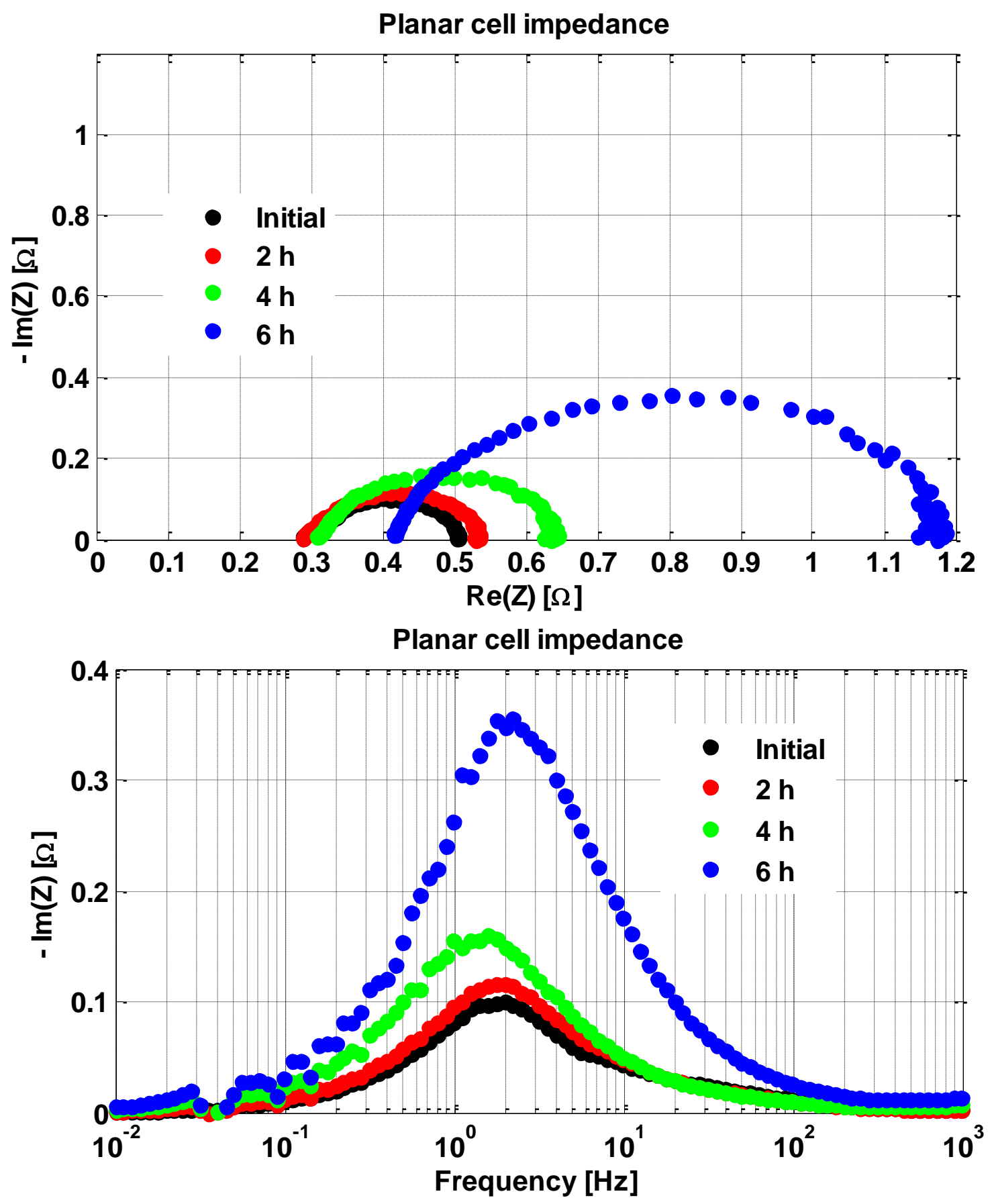

Figure A.8: Predicted impedance curves for planar cell at different time of phosphine exposure (initial, 2h, 4h, 6h): Nyquist representation (top) and bode representation (bottom) 


\section{APPENDIX B}

This work is submitted to journal of electrochemical acta

\section{Phosphine induced Nickel Migration in SOFC Anodes: A Computational Study}

\section{B.1 Abstract}

The poisoning of Solid Oxide Fuel Cell (SOFC) anode materials by impurities in coal syngas is a significant problem for utilization of coal syngas in SOFCs. One such impurity, phosphine is known to cause catastrophic failure of SOFC anode at ppm level concentrations. A significant phenomenon observed in SOFC anodes, made of Ni-YSZ cermets, when exposed to phosphine is migration of the nickel from porous matrix towards the surface, which is believed to be one of the reasons for performance degradation. The mechanisms responsible for the experimentally observed nickel migration are not well understood. A possible mechanism, which considers nickel diffusion and the formation of secondary phases (Ni-P compounds) as responsible phenomenon of nickel migration, was proposed by our group. However this consideration does not allow us to predict the experimentally observed effect of the applied current and steam concentration in the fuel. In this study, a more plausible mechanism is proposed to reveal the effect of electrical current and steam concentration on nickel migration in SOFC anodes. We postulate that the applied electrical current could possibly cause the transport of nickel by generating an electrical force, which is also a function of steam concentration, in the current flow direction. In SOFCs, the 
electrical current flows from anode/electrolyte interface to anode/fuel interface. Therefore the direction of the nickel transport is from anode active layer towards the anode surface. In addition, the source term of the secondary phases is proposed to be a function of the steam concentration guided by the experimental observations. Along these lines a physics based transport model for nickel migration is formulated and it is integrated into a readily available one dimensional in house code for predicting SOFC anode degradation due to fuel impurities. Simulations show that the proposed mechanism of $\mathrm{Ni}$ diffusion driven by secondary phase formation, the electrical force, and humidity can reveal the experimentally observed accumulation of $\mathrm{Ni}$ and secondary phases on the SOFC anode surface.

\section{B.2 Introduction}

Solid oxide fuel cells (SOFCs) are electrochemical devices which are operated at elevated temperatures $\left(700^{\circ} \mathrm{C}-900^{\circ} \mathrm{C}\right)$ with various fuels including gasified coal (syngas). Utilization of coal syngas in SOFCs can play a critical role in future power generation, with much less environmentally harmful emissions than conventional coal combustion [65]. However, the inherent contaminants found in coal syngas such as phosphorus, antimony, arsenic, zinc, sulfur, mercury, selenium, and vanadium may degrade the performance and durability of SOFC significantly even at low ppm levels [6]. The standard SOFC's anode composition is Ni-YSZ cermet which is affected by coal syngas contamination during cell operation. The effect of gasified coal contaminants on the Ni-YSZ anode, thus on the performance and durability of SOFCs has been an important research subject $[6,65,16]$. A significant phenomenon in SOFC Ni-YSZ cermets anodes exposed to phosphine is migration of the nickel from porous matrix to the surface, which is believed to be one of the reasons for performance degradation $[65,66,67]$. The performance 
degradation and/or Ni migration of Ni-YSZ anode due to the phosphine in syngas at low level (ppm levels) has been investigated by many research groups. Demircan et al. [66] reported the possible mechanism of phosphine induced performance degradation with two different mechanisms: (i) The loss of $\mathrm{Ni}$ in the active layer of the anode via nickel migration from anode active layer to the anode surface (ii) the production of Ni-P compounds decrease the number of active sites in Ni catalyst. Xu et al. [67] reported that, Ni can react with phosphine under cell operation conditions and form a liquid nickel phosphide phase which may possibly increase $\mathrm{Ni}$ migration from active layer to anode surface. Lussier et al. [65] reported the similar mechanism for Ni migration for hydrogen sulfide exposure. In addition to the formation of Ni-P phases, it has been reported that moisture and the electrical current also affect the nickel accumulation at anode surface $[20,68,69]$. Zhi et al. [68] \& Silva [69] had investigated the effect of the current on the nickel transport. They reported that the one possible cause of the nickel accumulation under the applied current at the anode surface is the electro migration. Celik et al. [20] run the cell under different applied current and steam concentration. It was observed by Celik et al. that both applied current and steam concentration accelerated the nickel transport towards the anode surface. However, the mechanism responsible for the experimentally observed Ni migration is still not well understood. The steam concentration has a direct effect on the electro migration [70] and the formation of the secondary phases $[19,20]$. Therefore, the formation of secondary phases and the electro migration due to the applied current seem to be the most reasonable mechanisms, which are possibly responsible for nickel migration.

Formation of secondary phases has been investigated by different groups. Lussier et al. [65] and Demircan et al. [66] observed that the chemical compound on anode surface can be $\mathrm{Ni}$ and $\mathrm{Ni}$ compound with impurities such as $\mathrm{H}_{2} \mathrm{~S}$ and $\mathrm{PH}_{3}$. Marina et al. [18] reported that the most possible 
species of Ni-P compounds are $\mathrm{Ni}_{3} \mathrm{P}, \mathrm{Ni}_{5} \mathrm{P}_{2}, \mathrm{Ni}_{12} \mathrm{P}_{5}$, and $\mathrm{Ni}_{2} \mathrm{P}$. Nickel conversion to nickel phosphide in the anode was considered to be one possible mechanism of SOFC performance degradation by Marina et al. [18]. Guo et al. [19] investigated the possible secondary phases formed due to phosphine exposure. Guo et al. [19] revealed the formation of secondary phases inside the anode and the dominant P-based phases were found to be nickel phosphates such as $\mathrm{Ni}\left(\mathrm{PO}_{3}\right)_{2}$ for a cell under load (e.g. $\left.0.3 \mathrm{~V}\right)$, however under open circuit voltage condition(OCV) the most possible secondary phase was found to be $\mathrm{Ni}_{3} \mathrm{P}$.

Electro migration has been recognized as an important mechanism which may lead to failure in electrical and electronic systems [70,71,72]. Electro migration was suggested to be one of the responsible mechanisms for the redistribution of elements in the Ni-YSZ SOFC anode under applied current $[68,73]$.

Based on the information found in the literature, we postulate that the nickel concentration gradients inside the anode could potentially cause $\mathrm{Ni}$ diffusion. Upon phosphine exposure, the $\mathrm{Ni}$ on the surface layer is the first to react and form secondary phases causing the Ni concentration to decrease further at the surface. This will drive the diffusion of Ni from the bulk of the porous anode to the surface. The melting point for some secondary phases that are reported by Marina et al. and Guo et al. $[19,18]$ is close to the usual cell operating temperature of $800^{\circ} \mathrm{C}$ causing them to soften and thus be more conducive for Ni migration. The effect of the current can be approximated as a convective term included by the electro migration mechanism as explained by Popovich et al. [74] for electrical and electronic systems.

Along these lines, a transport model for nickel migration is formulated and it is integrated with an existing one dimensional code [12] for predicting SOFC anode degradation due to fuel impurities. 
Interested readers are referred to $[12,16]$ for detailed information about $1 \mathrm{D}$ degradation model. In this work, an attempt is made to understand the elemental redistribution within the SOFC anode under $\mathrm{PH}_{3}$ exposure and the mechanism of Ni migrations by performing simulations at different current loading conditions. In addition, simulations were performed on SOFC anode to understand the influence of the steam concentration on the Ni migration phenomena.

\section{B.3 Theory and model equations}

\section{B.3.1 Integration with one dimensional degradation model}

The degradation model employed in this work considers the competitive adsorption of hydrogen and the phosphine on the nickel catalyst surface inside the anode. The assumed monolayer adsorption mechanism for the formation of secondary phases and hydrogen adsorption is described in $[12,16]$ and based on the following reactions:

$$
\begin{aligned}
& P H_{3}+N i \stackrel{\stackrel{k_{f, P H_{3}}}{\longrightarrow}}{\stackrel{k_{b, P H_{3}}}{\longrightarrow}} N i-P H_{3} \\
& H_{2}+2 N i \stackrel{k_{k_{b, H_{2}}} \stackrel{k_{f, H_{2}}}{\longrightarrow}}{\rightleftarrows} 2(N i-H)
\end{aligned}
$$

In eq. (B.1 and B.2) $k_{f, P H_{3}}, k_{b, P H_{3}}$, and $k_{f, H}, k_{b, H}$ are forward and backward reactions rates for formation of secondary phases and adsorption of hydrogen respectively. The one-dimensional phenomenological transport equation, $[12,16]$ for the coverage of phosphine transport inside the anode is:

$$
\frac{\partial \theta_{i}}{\partial t}=D_{\theta} \frac{\partial^{2} \theta_{i}}{\partial t^{2}}+\omega_{\theta_{i}}
$$


where $\theta_{i}$ is the surface coverage of phosphine. $D_{\theta}$ is the transport coefficient for the coverage which is taken as $1 \times 10^{-9} \mathrm{~m}^{2} / \mathrm{s}$ [12]. $\omega_{\theta_{i}}$ is the source term for formation of secondary phases. Detailed information on the performance degradation model can be found in Cayan et al. [12]. The dependency of porosity, electrical conductivity and exchange current density of hydrogen to local contaminant coverage is given as follows, [12,46];

$$
\begin{aligned}
& \varepsilon=\varepsilon_{0}\left(1-0.95 \theta^{p}\right) \\
& \sigma=\sigma_{0}\left(1-0.95 \theta^{p}\right) \\
& i_{o, H_{2}}=c_{1}\left(\frac{y_{H_{2}, \text { int }}}{y_{H_{2}, \text { ref }}}\right) \exp \left(-\frac{E_{a c t, H_{2}}}{R_{u} T}\right)\left(1-\theta_{N i-P H_{3}, \text { int }}^{m}\right)^{n}
\end{aligned}
$$

Where $\varepsilon_{0}, \sigma_{0}, \varepsilon, \sigma$ and $\theta$ are initial porosity, initial conductivity, porosity, conductivity and phosphine coverage, respectively. In eq. (B.5), $\mathrm{m}$ and $\mathrm{n}$ are the model parameters obtained from the available experimental data for button cells. The constant $C_{1}$ is taken as $3.936 \times 10^{7}$, the hydrogen reference concentration, $y_{H_{2}, r e f}$, is 0.333 , the hydrogen activation barrier, $E_{H_{2}, a c t}$ is $91.661 \mathrm{~kJ} / \mathrm{mol}$ [16], and $\mathrm{Ni}-\mathrm{PH}_{3}$, int is the phosphine coverage at the anode/electrolyte active interface. 


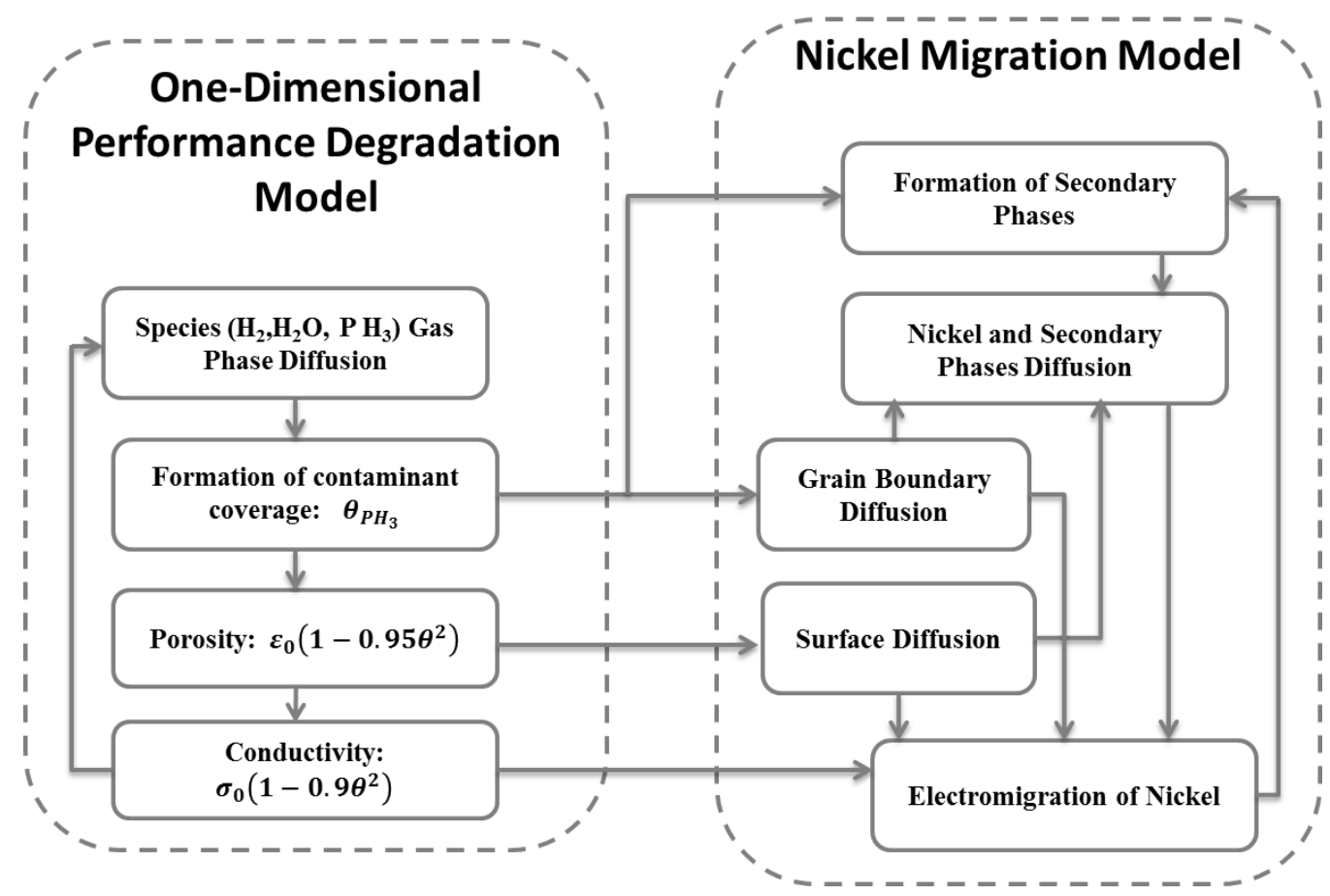

Figure B.1: Integration of nickel migration and one dimensional performance degradation code

A transport model developed to predict fuel contaminant induced elemental redistribution in SOFCs anodes is integrated into the one dimensional impurity induced degradation model developed by Cayan et al. [12]. The newly developed nickel migration model is composed of three parts (Figure B. 1), formation of secondary phases, species diffusion and transport by electro migration. The first part, formation of secondary phases, is interlinked with transport of contaminant coverage which is calculated from one dimensional degradation model. Porosity is calculated from Eq. B.1 and it is used to calculate the diffusion of nickel and secondary phases inside the anode. 


\section{B.3.2 Diffusion and formation of secondary phases}

The random motion of atoms of a solid material become intense at the temperatures close to melting point. This random motion can happen in three different regions: surface, grain boundary, volume (lattice), inside the solids.

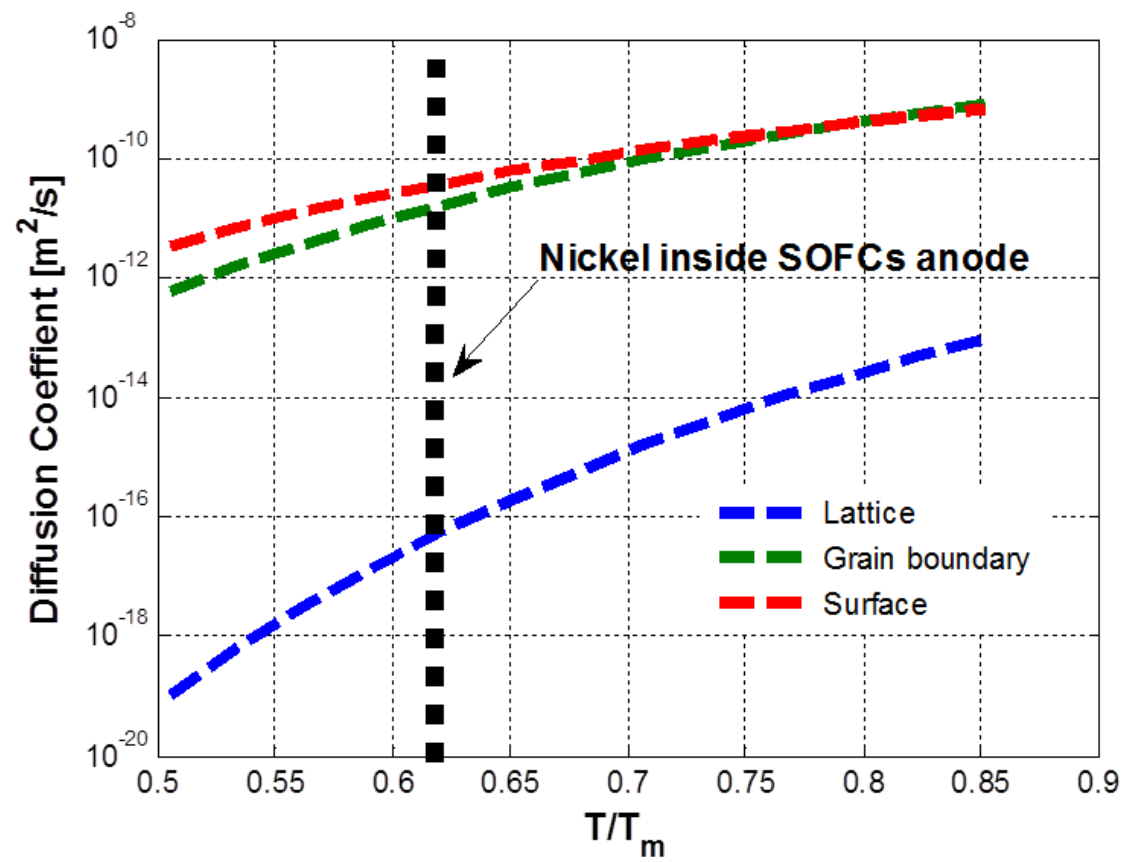

Figure B.2: Nickel diffusion coefficients from lattice, grain boundaries and surfaces at high temperatures.

At elevated temperatures $\left(0.3 \mathrm{~T}_{\mathrm{m}}<\mathrm{T}<\mathrm{T}_{\mathrm{m}}\right)$ grain boundary and surface diffusion are sufficiently high to provoke the transport of the metals inside the solids due to the atomic concentration gradients [75]. In Figure B. 2 the magnitudes of different diffusion coefficients are given. In this study, it is assumed that SOFCs operating temperature (T/T $\mathrm{m}$ Nikel $=0.62$, Figure B. 2) promote grain boundary and surface diffusion inside the porous anode; hence it is counted as one of responsible mechanisms from nickel migration. Diffusion coefficients for face centered cubic 
(FCC) metals, $D_{\text {lattice }}, D_{\text {grain boundary }}, D_{\text {surface }}$, are calculated and plotted in Figure B. 2 based on the following formulations that are given by $\mathrm{Tu}$ et al. [76];

$$
\begin{gathered}
D_{\text {lattice }}=5 \times 10^{-5} e^{\left(-34 T_{m} / R T\right)} \\
D_{\text {grain boundary }}=3 \times 10^{-5} e^{\left(-17.8 T_{m} / R T\right)} \\
D_{\text {surface }}=1.4 \times 10^{-7} e^{\left(-13 T_{m} / R T\right)}
\end{gathered}
$$

Where $\mathrm{T}_{\mathrm{m}}, 1728^{\circ} \mathrm{K}$, is the melting point of nickel, $\mathrm{R}\left(\mathrm{Cal} / \mathrm{mole}-{ }^{\circ} \mathrm{K}\right)$ is universal gas constant and $\mathrm{T}\left({ }^{\circ} \mathrm{K}\right)$ is the SOFC operating temperature. The effective diffusion coefficient of the $\mathrm{i}^{\text {th }}$ specie, $D_{i}^{\text {eff }}$, is calculated as a combination of both surface and grain boundary diffusions from

$$
D_{i}^{e f f}=D_{i}^{g b}+D_{i}^{s}
$$

Where $D_{i}^{g b}$ is the diffusion coefficient for grain boundary diffusion; it is calculated from

$$
D_{g b}^{e f f}=\theta_{x} D_{g b 0}
$$

Here $\theta_{x}$ is contaminant coverage and $D_{g b 0}$ is the initial diffusion coefficient for grain boundary diffusion. As it is shown in Figure B. 2, surface diffusion is of the same order of magnitude as the grain boundary diffusion. The SOFC anode is highly porous; therefore the porous surfaces and open surface of anode can be considered as surface diffusion paths. The effect from the lateral open surface is neglected. With this assumption $D_{s}^{\text {eff }}$, the surface effective diffusion can be calculated from,

$$
D_{s}^{e f f}=\left(1-\frac{Z}{L}\right)^{m} D_{s 0}+\varepsilon D_{s 0}
$$


Where $\mathrm{z}$ is the distance from anode surface and $\mathrm{L}$ is the anode thickness. $D_{s 0}$ is the initial diffusion coefficient for the surface diffusion. By using Eqs. B.7-9, diffusion flux for any specie can be calculated as a function of mass concentration gradient and diffusion coefficient as follow;

$$
J_{D i f f}=-D_{i}^{e f f} \frac{\partial C_{N i}}{\partial z}
$$

This diffusion mechanism is an empirical treatment to capture the experimental observation [85]. The following solid state reaction is proposed for the formation of secondary phases. Here it should be noted that, formation of one mole of Ni-P phase consumes only one mole nickel. Namely, mass of the secondary phases is assumed to be equal to the mass of the nickel content inside the secondary phases.

$$
\left.N i+\theta_{x} \underset{k_{b}}{\stackrel{k_{f}}{\rightarrow}} N i-P \quad \text { (Secondary Phases }\right)
$$

The source term, $S_{N i-P}$, for the formation of the secondary phases can be written as a function of local Nickel mass concentration and $\mathrm{H}_{2} \mathrm{O}$ mole fraction inside the anode;

$$
S_{N i-P}=C_{N i} k_{f} *\left(y_{H_{2} O}\right)^{\alpha} \theta_{x}-C_{N i} k_{b}
$$

where $k_{f}$ and $k_{b}$ are the forward and backward reaction rate constants. $y_{\mathrm{H}_{2} \mathrm{O}}$ is mole fraction of the steam and $\alpha(=0.5$ in this study $)$ is a model parameter.

\section{B.3.3 Electro-migration}

Electro-migration is the transport of the charged atoms in a conductor due to the local electrical field and the momentum transfer between charged atoms and electrons, which are flowing inside 
the conductive medium $[70,74,77]$. It can cause failure of the electronic circuits by creating opens and shorts between the interconnects and the circuit elements [70] . Electro-migration in thin films and integrated circuit interconnects has been an intense study area, both theoretical and experimental, over the past three decades years [77]. In many studies [77,72,78], it is mentioned that the electro-migration can be effective under high current densities (i.e. $\mathrm{i}>10^{4} \mathrm{~A} / \mathrm{cm}^{2}$ ). Krumbein [70] and Tu [76] have studied the effect of the operating conditions on the electromigration. According to Krumbein [70] the following operating conditions may accelerate the electro-migration: 1) moisture, 2) contaminant on the insulator surface, 3) voltage difference between conductors, 4) narrow spacing widths and 5) elevated temperatures. The effect of these factors on electro-migration is discussed in $[79,80,70]$. Krumbein stated that at elevated temperature, contaminants and under the steam concentration (humidity or visible moisture, i.e. wet electro-migration), the electro-migration can happen at low current densities (i.e. $\mathrm{i}<1$ $\left.\mathrm{mA} / \mathrm{cm}^{2}\right)[72,81,82]$. SOFCs are operated at aforementioned operating conditions, which can promote the electro-migration at low current densities.

The electro-migration phenomenon is primarily derived by two physical forces, electron wind force due to the momentum transfer and electrical force due to the electrical field [70]. The schematic illustration of these two forces on charged particles is shown in Figure B. 3. Electrons move under a potential difference, which generate an electric field on the reverse direction of the electron flow. The electrons move faster under high potential difference and temperature conditions. High velocity electrons interact with the metal ions in the conductive medium and due to this interaction electrons transfer a part of their momentum to the metal ions and this is the so called electron wind force. Positive charged metal ions in an electric field moves by electrical force on the direction of applied electric field. Electrons under the electric field move in the reverse 
direction of the electric field (Figure B. 3). The net force is the difference between these two forces. Because of the shielding effect of the electrons interaction with metal ions, the electron wind force dominates $[83,78]$. This means that in an electron conductive medium a mass flux of ions exists and it is parallel to the electron wind force. This mass flux is the driving force behind the electromigration and it is given as a function of mass concentration, current density, steam concentration and effective diffusion coefficient expressed by the following equation [74]:

$$
J_{N i}=-C_{N i} a\left(\frac{Z e \rho j}{R T} D_{N i}^{e f f}\right)\left(y_{H_{2} O}\right)^{\beta}
$$

Where $J_{i}$ and $C_{i}$ are the net ion mass flux and mass per volume of ith specie, respectively. $Z e, \rho$, and $j$ are charge of metal ions, electrical resistivity of the conducting medium (SOFCs anode) and current density, respectively. $D_{i}^{e f f}$ is the effective diffusion, which is given in the previous section as a combination of grain boundary and surface diffusion for each specie. The constant coefficient, a (in the range: $10^{3}-10^{4}$ ), represents the effect of the SOFC's operating conditions, (i.e. elevated temperature, porous structure, contaminants, steam, mechanical stresses), $[70,84])$ which promote severe electro-migration. The exponent, $\beta(=1)$, is a model parameter used to account for the influence of the steam concentration. These parameters can be calibrated against experiments if necessary. Using dimensional analysis on the mass flux equation, a velocity term, usually called drift velocity for atomic flux, can be can be calculated as;

$$
u=a\left(\frac{Z e \rho j}{R T} D_{N i}^{e f f}\right)\left(y_{H_{2} O}\right)^{\beta}
$$

Using eq.14, the mass flux equation yields;

$$
J_{N i}=-u C_{N i}
$$




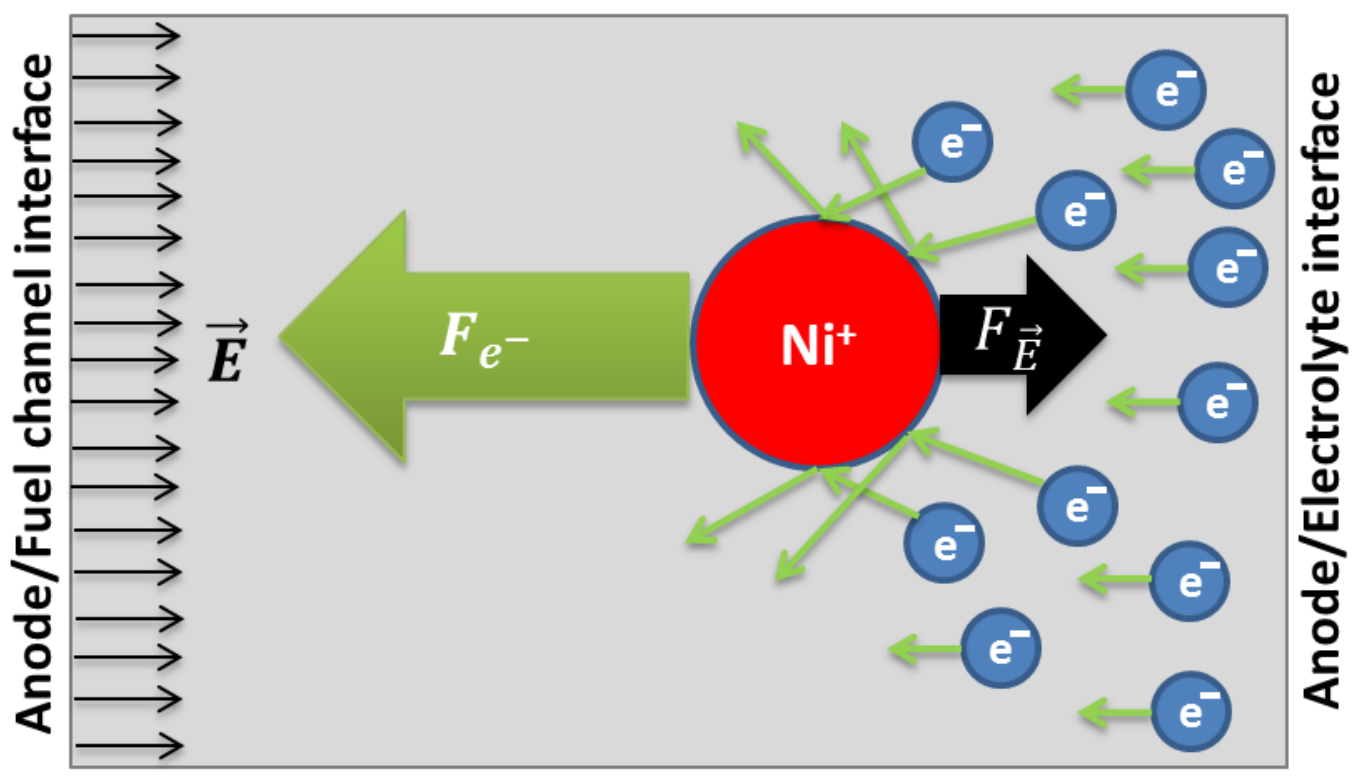

Figure B.3: Charged ions transport mechanism under the electron wind force due to the momentum transfer and electrical force due to the electrical field

\section{B.3.4 Solids Transport Equation}

The porous anode of a SOFC undergoes through four main stages as it is shown in Figure B. 4 The initial stage is Ni-YSZ without exposure to any contaminant Figure B. 4a. In the second stage (Figure B. 4b) anode is exposed to contaminant and the contaminant coverage propagates inside anode. Figure B. $4 \mathrm{c}, 3^{\text {rd }}$ stage, shows the formation of secondary phases which are compounds of $\mathrm{Ni}, \mathrm{P}, \mathrm{H}$, and $\mathrm{O}$. The last stage of SOFC's anode is that causes the eventual cell failure (Figure B. 4d). As it is illustrated in Figure B. 4d, the amount of secondary phases and nickel at open surface is higher than the rest of anode. It should be noted that nickel migration phenomenon is considered to be with the nickel atomic diffusion, electro-migration, and the formation of the secondary phases inside the anode. In the current model, spatial variation of nickel along the anode is calculated as the summation of atomic $\mathrm{Ni}$ and $\mathrm{Ni}$ contained within the secondary phases. 


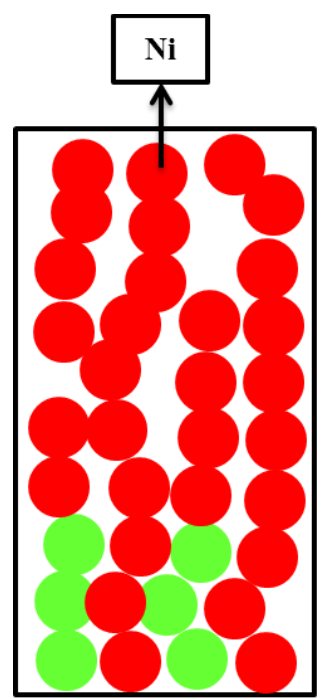

(a)

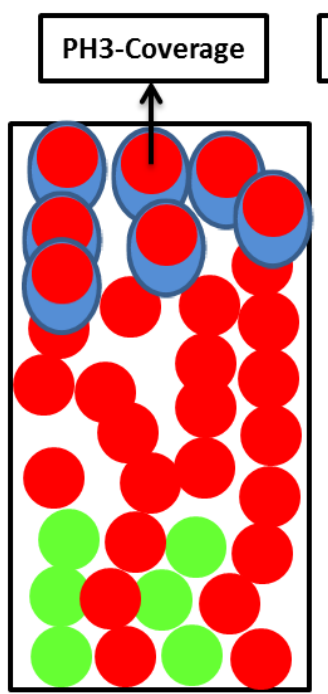

(b)

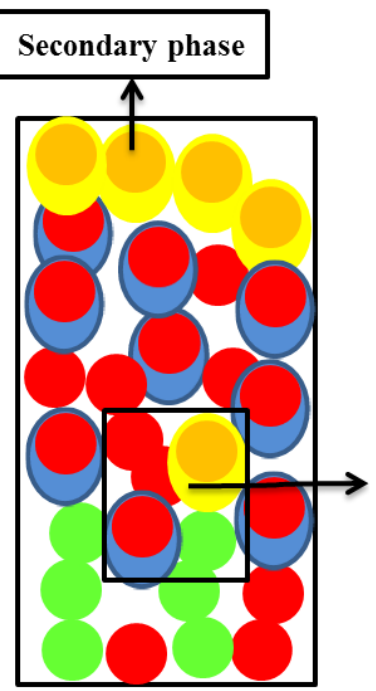

(c)

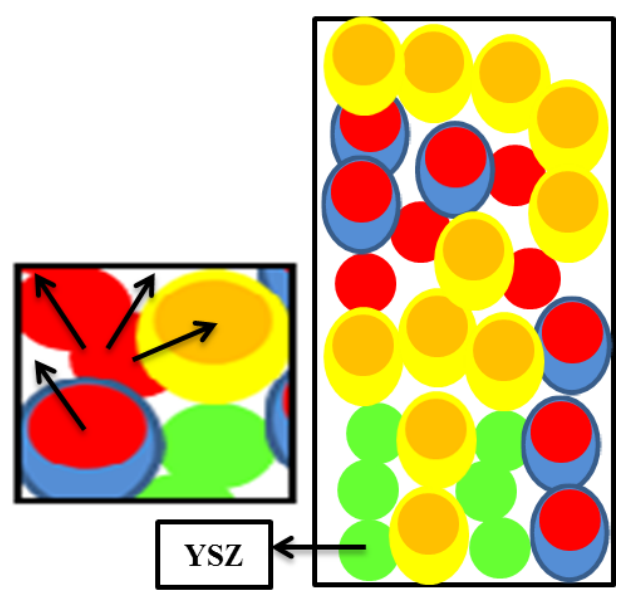

(d)

Figure B.4: (a) First stage of Ni-YSZ anode (b) second stage: formation of coverage (c) third stage: formation of secondary phases (d) fourth stage: nickel and secondary phases diffusion

Based on the observation that the front of the nickel and secondary phases concentrations move with time, a one dimensional phenomenological diffusion equation for the mass concentration propagation along the thickness of the anode for secondary phases is used in the form given in Eq. (16-17).

$$
\begin{array}{ll}
\frac{\partial C_{N i-p}}{\partial t}=\frac{\partial}{\partial z}\left(D_{N i-P}^{e f f} \frac{\partial C_{N i-P}}{\partial z}\right)+S_{N i-P} & \text { Secondary Phases } \\
\frac{\partial C_{N i}}{\partial t}+\frac{\partial}{\partial z}\left(u C_{N i}\right)=\frac{\partial}{\partial z}\left(D_{N i}^{e f f} \frac{\partial C_{N i}}{\partial z}\right)-S_{N i-P} & \text { Nickel(Diffusion+Electro-migration) }
\end{array}
$$

Where, $S_{N i-P}$, source term is due to the formation of the secondary phases which works as a sink source for nickel. The sources terms, $\mathrm{S}$, are a function of contaminant concentration which can be identified with one-dimensional degradation [12] model along the anode. The effect of the electromigration is added as a convective term for the nickel mass transport Eq. 17. Zirconium ions migration was observed experimentally by Celik et al. [20]. However the elemental redistribution 
of the zirconium atom was relatively small compared to the nickel elemental redistribution under the SOFC operating conditions. Therefore the transport of the zirconium is neglected in this study.

\section{B.3.5 Computational approach}

The one dimensional SOFC's anode geometry used in the simulations is shown in Figure B. 5a. A finite volume method approach is used to discretize the transport equation for the model [60]. Discretized computational control volume cell is given in Figure B. 5b. It should be noted that the effective diffusion coefficient and the drift velocity in the transport equations vary with the spatial distance and the time. Diffusion coefficient is calculated at each finite volume node and time step during the simulation. Since the mass flux due to the convection term is defined at faces of computational cell, the drift velocity is calculated at those faces.

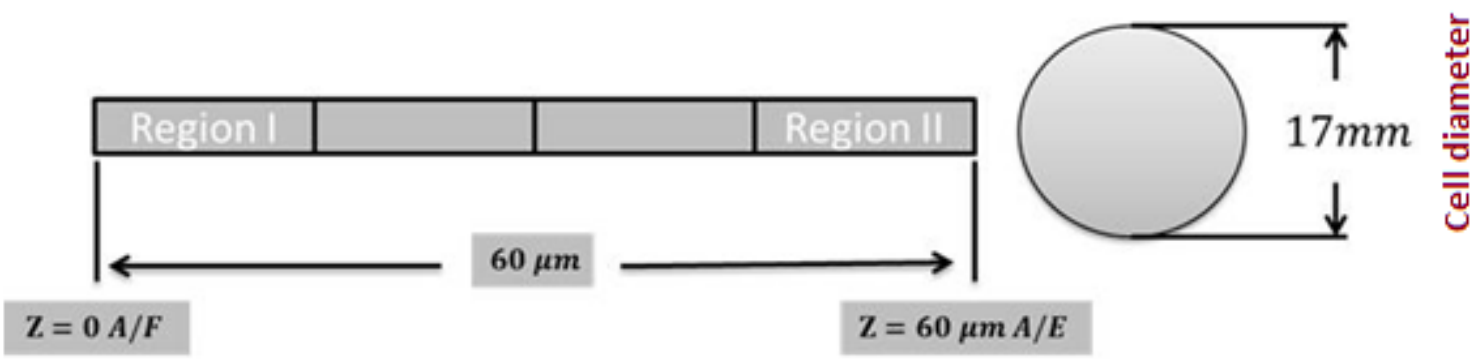

(a)

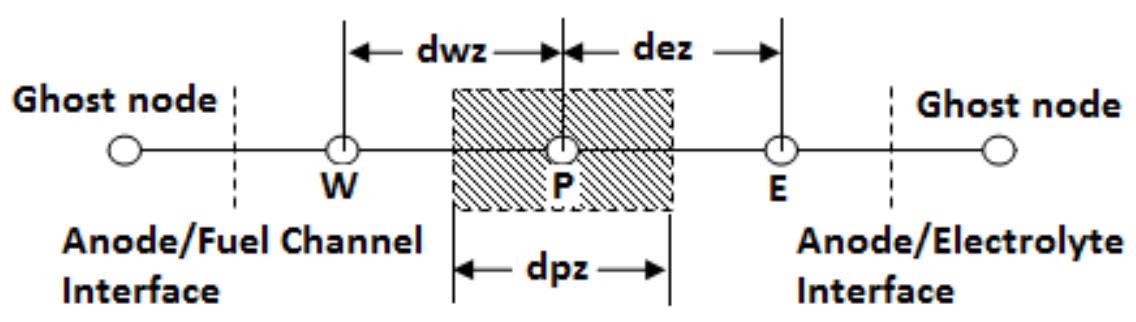

(b)

Figure B.5: (a) One dimensional SOFC's anode geometry, (b) Discretization of computational domain

The detailed geometric parameters and operation conditions for the all simulations in this study are given in Table B. 1. Simulations have been performed under different operating conditions to 
understand the influence of the current and steam concentration on elemental redistribution. A $60 \mu \mathrm{m}$ thick Ni/YSZ cermet (\%50 wt) SOFC's anode was considered. Initial species and porous volume variation along the anode is calculated based on the following equations;

$$
\varepsilon_{0}=\left\{\left(1-\frac{Z}{L}\right)^{c_{1}}\right\}^{c_{2}}\left(\varepsilon_{0, i s l}-\varepsilon_{0, i a l}\right)+\varepsilon_{0, i a l}
$$

Where $\varepsilon_{0, i s l}$ and $\varepsilon_{0, i a l}$, are initial porosity at support and active layers and $\varepsilon_{0}$ is initial porosity of anode. $\mathrm{z}$ is the distance from Anode/fuel interface and $\mathrm{L}$ is anode thickness. $\mathrm{c}_{1}=13.0$ and $\mathrm{c}_{2}=9.0$ are constants, which are used to smooth the step between anode support layer and active layer porosities.

Table B.1: Conditions and parameters for simulations

\begin{tabular}{|c|c|c|c|}
\hline \multicolumn{4}{|c|}{ Model Parameters and operating conditions } \\
\hline Temperature, ${ }^{\circ}$ & 800 & $\mathrm{q}$ & 2 \\
\hline $\mathrm{m}$ & 2 & $\mathrm{a}$ & $10^{4}$ \\
\hline $\mathrm{n}$ & 7 & $\mathrm{a}_{1}, \mathrm{a}_{2}$ & $13.0,9.0$ \\
\hline $\mathrm{p}$ & 2 & $\alpha$ & 0.5 \\
\hline$\gamma$ & 5 & $\beta$ & 1 \\
\hline \multicolumn{2}{|c|}{$\mathrm{k}_{\mathrm{f}}, \mathrm{k}_{\mathrm{b}}, \quad \operatorname{gram} /\left[\mathrm{sec} \cdot \mathrm{m}^{3}\right]$} & \multicolumn{2}{|c|}{$10^{-4}, \quad 5.48 \times 10^{-11}$} \\
\hline \multicolumn{2}{|c|}{ Anode support layer initial porosity (\%) } & \multicolumn{2}{|c|}{0.3616} \\
\hline \multicolumn{2}{|c|}{ Anode active layer initial porosity (\%) } & \multicolumn{2}{|c|}{0.1616} \\
\hline \multicolumn{2}{|c|}{ Initial Electrical Conductivity (S/m) } & \multicolumn{2}{|c|}{520.97} \\
\hline \multicolumn{2}{|c|}{ Initial YSZ mass (gram) } & \multicolumn{2}{|c|}{0.031767} \\
\hline \multicolumn{2}{|c|}{ Initial Ni mass (gram) } & \multicolumn{2}{|c|}{0.031767} \\
\hline \multirow{4}{*}{ Case studies } & $\operatorname{wet}\left(0.25 \mathrm{~A} / \mathrm{cm}^{2}\right)$ & \multicolumn{2}{|c|}{$75 \% \mathrm{H}_{2}, 25 \% \mathrm{H}_{2} \mathrm{O}, 10 \mathrm{ppm} \mathrm{PH}_{3}, \mathrm{i}=0.25 \mathrm{~A} / \mathrm{cm}^{2}$} \\
\hline & wet $(\mathrm{OCV})$ & \multicolumn{2}{|c|}{$75 \% \mathrm{H}_{2}, 25 \% \mathrm{H}_{2} \mathrm{O}, 10 \mathrm{ppm} \mathrm{PH}_{3}, \mathrm{i}=0 \mathrm{~A} / \mathrm{cm}^{2}$} \\
\hline & $\operatorname{dry}\left(0.25 \mathrm{~A} / \mathrm{cm}^{2}\right)$ & \multicolumn{2}{|c|}{$100 \% \mathrm{H}_{2}, 0 \% \mathrm{H}_{2} \mathrm{O}, 10 \mathrm{ppm} \mathrm{PH}_{3}, \mathrm{i}=0.25 \mathrm{~A} / \mathrm{cm}^{2}$} \\
\hline & $\operatorname{dry}(\mathrm{OCV})$ & \multicolumn{2}{|c|}{$100 \% \mathrm{H}_{2}, 0 \% \mathrm{H}_{2} \mathrm{O}, 10 \mathrm{ppm} \mathrm{PH}_{3}, \mathrm{i}=0 \mathrm{~A} / \mathrm{cm}^{2}$} \\
\hline
\end{tabular}




\section{B.4. Results and Discussions}

Initial species volume, Ni, YSZ, and pores, and nickel mass distribution is depicted in Figure B. 6a. A mixture of hydrogen, steam and phosphine was supplied to the anode side, and initial nickel mass along the anode shown in Figure B. $6 \mathrm{~b}$ was used. The values for the model parameters, $m, n, p, q$, involved in the one-dimensional performance degradation model were obtained from our previous work [46] in which $\mathrm{SOFC}$ anode was exposed to $10 \mathrm{ppm} \mathrm{PH}_{3}$. Cell potential drop and phosphine coverage at gradual degradation times are predicted under phosphine exposure by using these set of the parameters (Figure B. 7a\&b) in the degradation model.
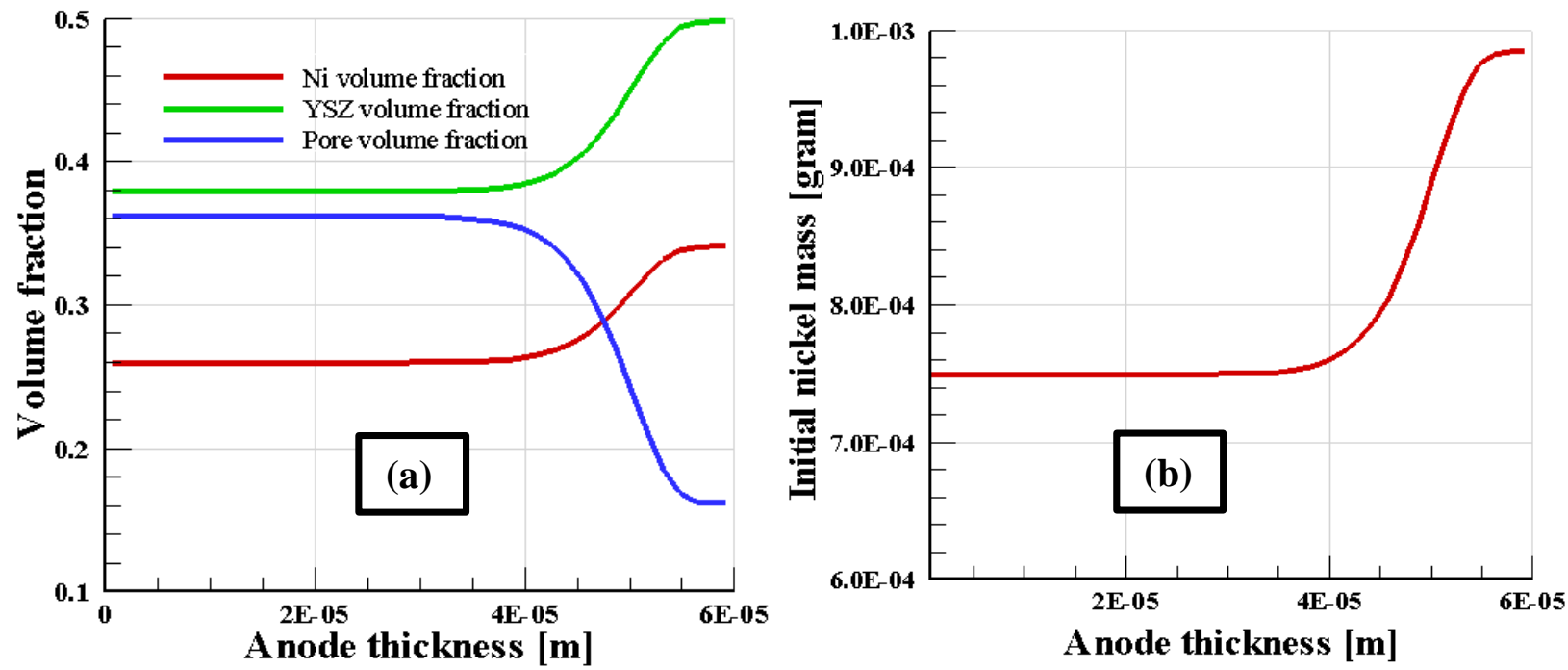

Figure B.6: Initial conditions of anode (a) initial spatial species and porous volume distribution along anode. (b) Initial spatial Nickel mass (gram) distribution along anode.

Here time $\mathrm{t}=0$ is the time at which the $\mathrm{PH}_{3}$ injection has started. It can be seen from the simulated degradation curve (Figure B. 7a) that initially the degradation process is slow, however the subsequent sharp potential drop started due to the coverage acceleration at the active layer, which causes severe activation losses (Figure B. 7a\&b). 

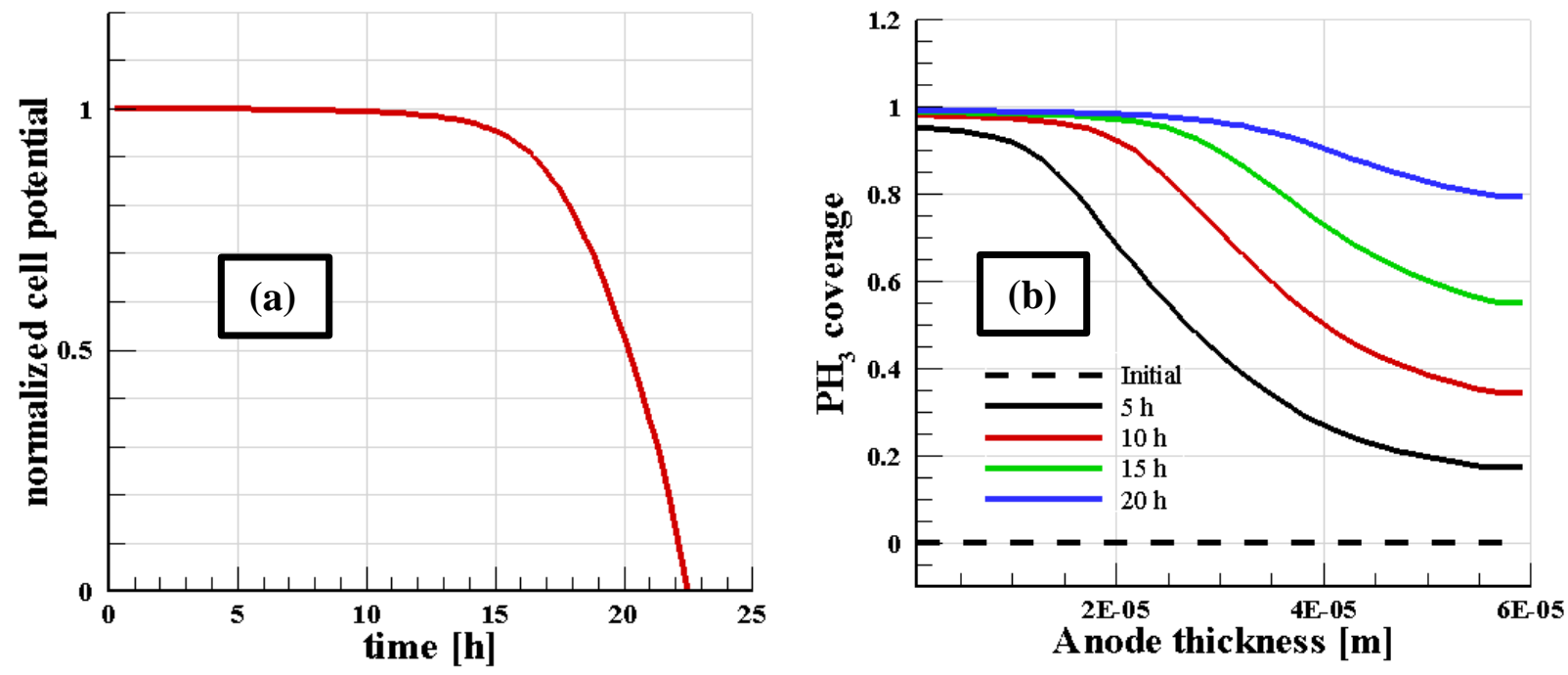

Figure B.7: Wet (\%25) fuel case; performed for SOFCs with $60 \mu \mathrm{m}$ thick anode exposed to 10 ppm $\mathrm{PH}_{3}$ under $0.25 \mathrm{~A} / \mathrm{cm}^{2}$ current loading (a) Predicted cell potential drop (b) Phosphine coverage distribution at different gradual degradation time

The variation of porosity and electrical conductivity along the anode thickness at different exposure times are depicted on Figure B. 8a\&b respectively. The strong functional dependency of porosity and electrical current on the coverage can be observed from Figure B. $7 \mathrm{~b}$ and Figure B. $8 \mathrm{a} \& \mathrm{~b}$. The trace element coverage steadily increases over the time, it penetrates from anode surface towards the active interface, and the porosity decreases accordingly, thus surface diffusion of $\mathrm{Ni}$ element decreases. In contrast, grain boundary diffusion, a function of contaminant coverage, increases (Figure B. 9a\&b). In addition, electrical conductivity decreases accordingly when the trace element coverage propagate towards to anode interface, hence the electrical resistance, reciprocal of the electrical conductivity, increases. The atomic nickel drift velocity at different exposure times, as a function of the loading current, steam concentration, electrical resistance, grain boundary and surface diffusion, is depicted in Figure B. 10a. 

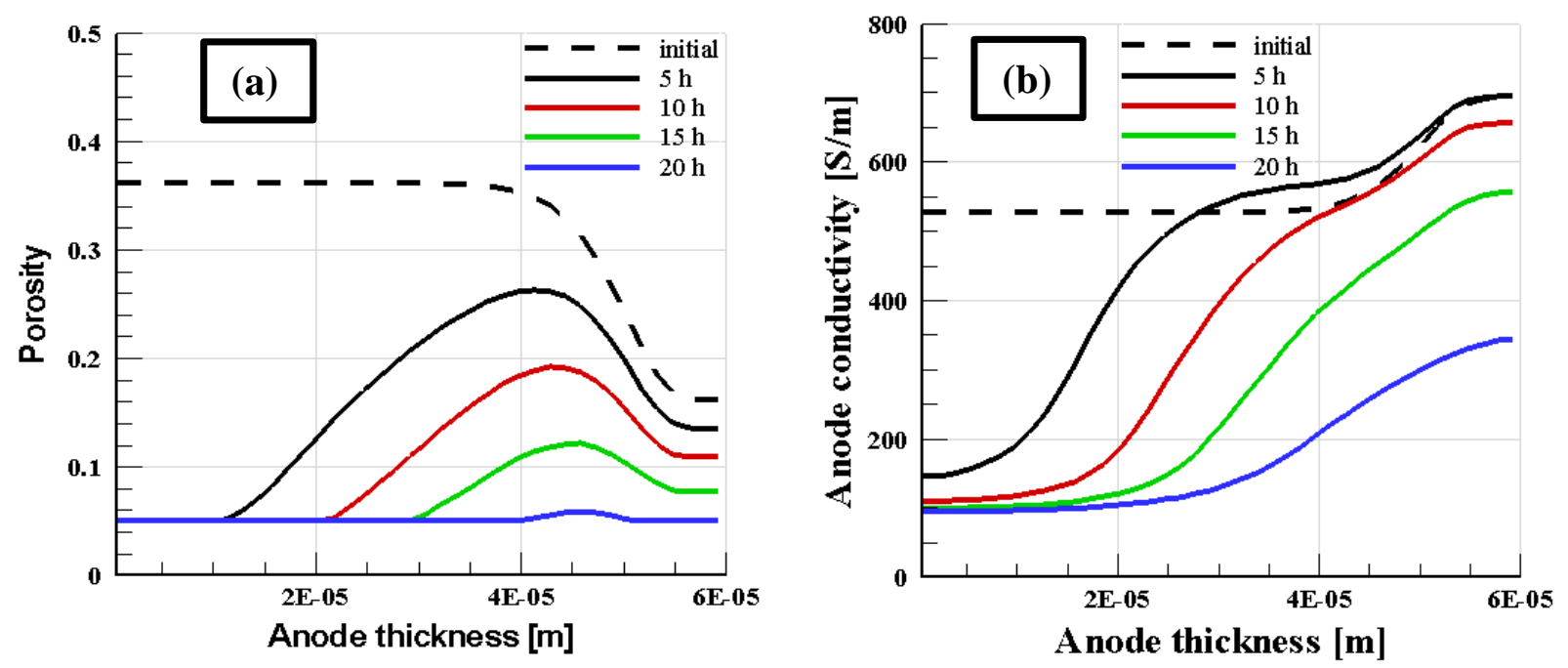

Figure B.8: Wet (\%25) fuel case; performed for SOFCs with $60 \mu \mathrm{m}$ thick anode exposed to 10 ppm $\mathrm{PH}_{3}$ under $0.25 \mathrm{~A} / \mathrm{cm}^{2}$ current loading (a) anode porosity and (b) anode conductivity distribution at different gradual degradation time

As it can be seen (Figure B. 8b), when electrical conductivity decreases upon phosphine exposure, the electrical resistance, hence the drift velocity, which is a convection term in scalar transport equation, increases as $\mathrm{PH}_{3}$ propagate inside anode in time (Figure B. $8 \mathrm{~b} \&$ Figure B. 10a). The functional dependency of drift velocity on the diffusion coefficients, surface and grain boundary, can be observed from Figure B. 9a\&b and Figure B. 10a. It is also shown that as grain boundary diffusion steadily increases over the time, the surface diffusion is dominant towards to anode/fuel interface, the drift velocity increases steadily and it is dominant near the anode/electrolyte interface. The Nickel atom accumulation under the calculated drift velocity is shown in (Figure B. 10b). Electro-migration was considered as a possible mechanism for the elemental redistribution of the anode under the phosphine exposure in [67] and [73]. In Figure B. 10a\&b, it is seen that the drift velocity, which is the main factor in electro-migration, influences the nickel accumulation near the anode/fuel interface steadily over the degradation time. 

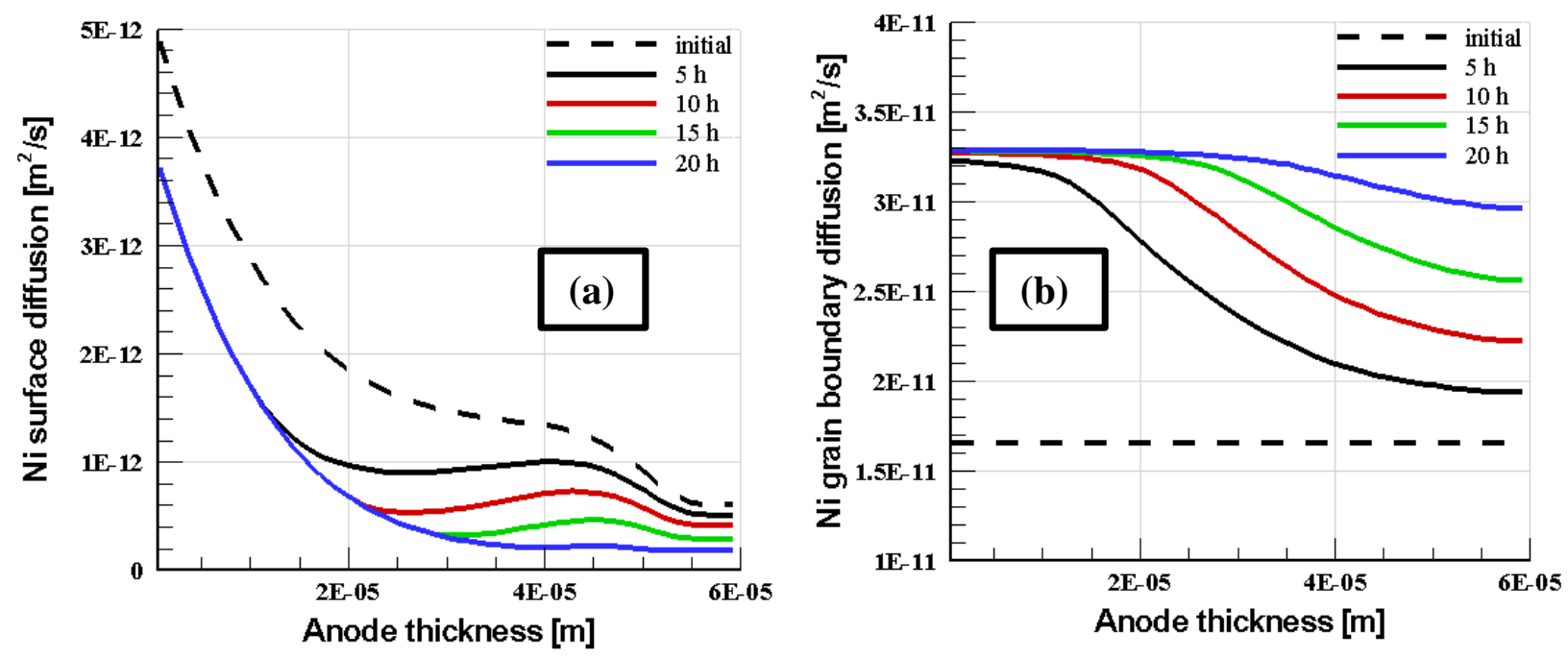

Figure B.9: Wet (\%25) fuel case; performed for SOFCs with $60 \mu \mathrm{m}$ thick anode exposed to $10 \mathrm{ppm}$

$\mathrm{PH}_{3}$ under $0.25 \mathrm{~A} / \mathrm{cm}^{2}$ current loading (a) surface diffusion inside anode (b) Spatial distribution of grain boundary diffusion at different gradual degradation time
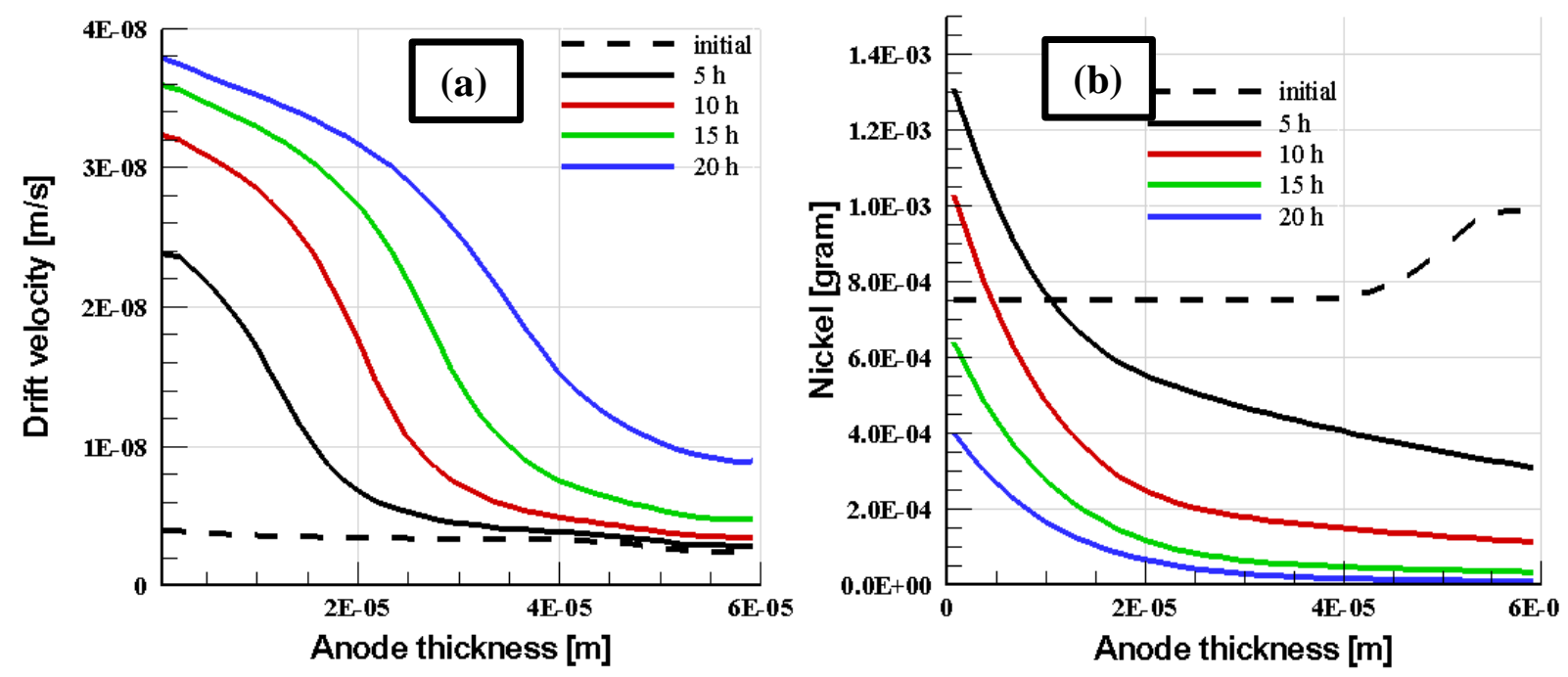

Figure B.10: Wet $(\% 25)$ fuel case; performed for SOFCs with $60 \mu \mathrm{m}$ thick anode exposed to 10 ppm $\mathrm{PH}_{3}$ under $0.25 \mathrm{~A} / \mathrm{cm}^{2}$ current loading (a) variation of drift velocity inside anode (b) Spatial distribution of Nickel at different gradual degradation time 

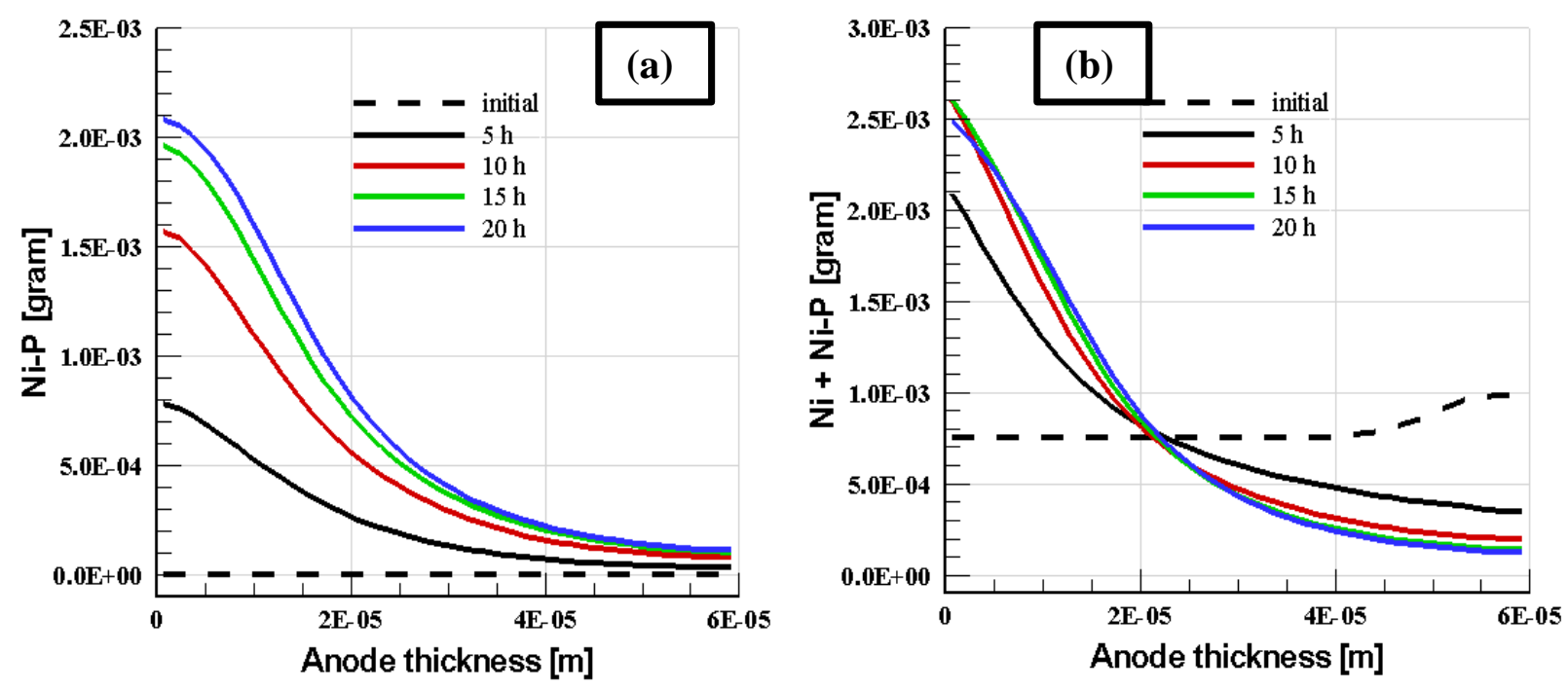

Figure B.11: Wet (\%25) fuel case; performed for SOFCs with $60 \mu \mathrm{m}$ thick anode exposed to $10 \mathrm{ppm} \mathrm{PH}_{3}$ under $0.25 \mathrm{~A} / \mathrm{cm}^{2}$ current loading (a) Ni-P phases (b) sum of $\mathrm{Ni}$ and $\mathrm{Ni}-\mathrm{P}$ phases at different gradual degradation time along anode thickness

In Figure B. 10 b, it is also seen that the spatial distribution of nickel has become more uniform, namely the concentration gradient of the $\mathrm{Ni}$ decreases, during the operation time, which means nickel has been also redistributed by nickel diffusion inside the anode. Formation of the secondary phases (Ni-P) and the sum of the $\mathrm{Ni}$ and $\mathrm{Ni}$ atoms in Ni-P phases over the time of phosphine exposure along the anode thickness is shown in Figure B. 11a\&b. Ni-P phases source term is a function of the available active Ni element, phosphine coverage, and present steam concentration. These functional relations between active Ni elements and Ni-P phases formation are clearly shown as the Ni elements move towards anode/fuel interface and is depleted by the Ni-P phases formation steadily over the degradation time. Ni consumption by the formation of the secondary phases decelerates the Ni-P phases formation along the anode with exposure time (Figure B. 10b \& Figure B. 11a). In addition, as the phosphine coverage increases, the rate of formation of Ni-P increases accordingly (Figure B. 11 a\& Figure B. 7b). However, the rate of the coverage is higher 
than that of the Ni-P phases. This difference on the rate of the formation is due to aforementioned $\mathrm{Ni}$ consumption. In addition, as it is expected, the sum of the $\mathrm{Ni}$ and $\mathrm{Ni}-\mathrm{P}$ phases show both the influence of the Ni-P formation and Ni elemental transport and depletion (Figure B. 10b, Figure B. 11a\&b). As it is seen in Figure B. 11b, Ni content near the anode free surface almost reaches a steady state trend. This behavior is due to the diffusion of the secondary phases. The amount of secondary phases increases rapidly towards to anode surface; hence the concentration gradient of these phases increases, therefore the diffusion of Ni-P phases play a role. This effect will be further discussed.

\section{B.4.1 The influence of the Steam Concentration and Loading Current}

In order to reveal the effect of the steam concentration and loading current, additional simulations, dry at $0.25 \mathrm{~A} / \mathrm{cm}^{2}$, wet $\left(25 \% \mathrm{H}_{2} \mathrm{O}\right)$ at $0.25 \mathrm{~A} / \mathrm{cm}^{2}$ and $\mathrm{OCV}\left(25 \% \mathrm{H}_{2} \mathrm{O}\right)$ and wet $\left(3 \% \mathrm{H}_{2} \mathrm{O}\right)$ at 0.25 $\mathrm{A} / \mathrm{cm}^{2}$ and $\mathrm{OCV}\left(3 \% \mathrm{H}_{2} \mathrm{O}\right)$, were performed. Inlet fuel concentration and model parameters used are listed in Table B. 1.

For these calculations, the SOFC anode is divided into 4 sections (Figure B. 5a) to calculate the accumulation and depletion of the Ni, Ni-P compounds, and Ni+Ni-P at anode/fuel (region I) and anode/electrolyte (region II) interfaces. The percentage accumulation of each species and the sum of the species in depicted regions are calculated as follows;

$$
\begin{aligned}
& \% N i_{I, I I}=\frac{\sum_{i=1}^{n} C_{N i}}{\sum_{i=1}^{N} C_{N i_{\text {init }}}} \\
& \% N i-P_{I, I I}=\frac{\sum_{i=1}^{n} C_{N i-p}}{\sum_{i=1}^{N} C_{N i_{\text {init }}}} \\
& \% N i+N i-P_{I, I I}=\frac{\sum_{i=1}^{n} C_{N i+N i-p}}{\sum_{i=1}^{N} C_{N i_{\text {init }}}}
\end{aligned}
$$


Here $C_{N i}, C_{N i-p}, C_{N i+N i-p}$ are the mass concentrations of the Ni, Ni-P and Ni+Ni-P compounds, respectively. $C_{N i_{i n i t}}$ represents the initial nickel mass concentration inside the anode. The sum of the initial Ni mass concentration is equivalent to the mass concentration of the $\mathrm{Ni}+\mathrm{Ni}-\mathrm{P}$ compound at any instant during degradation time inside the whole anode. This is assured by using a conservative finite volume method for the numerical solution of the scalar transport equation. Symbols I and II represent region I and II. In Eq. B.20, N (=41), and n (=10), are the number of the computational grid nodes in the whole anode and each region, respectively. The accumulation of $\mathrm{Ni}, \mathrm{Ni}-\mathrm{P}$ and $\mathrm{Ni}+\mathrm{Ni}-\mathrm{P}$ compounds in region I and II after phosphine exposure at different current and steam concentration conditions are presented in Figure B. 12-17 a\&b.
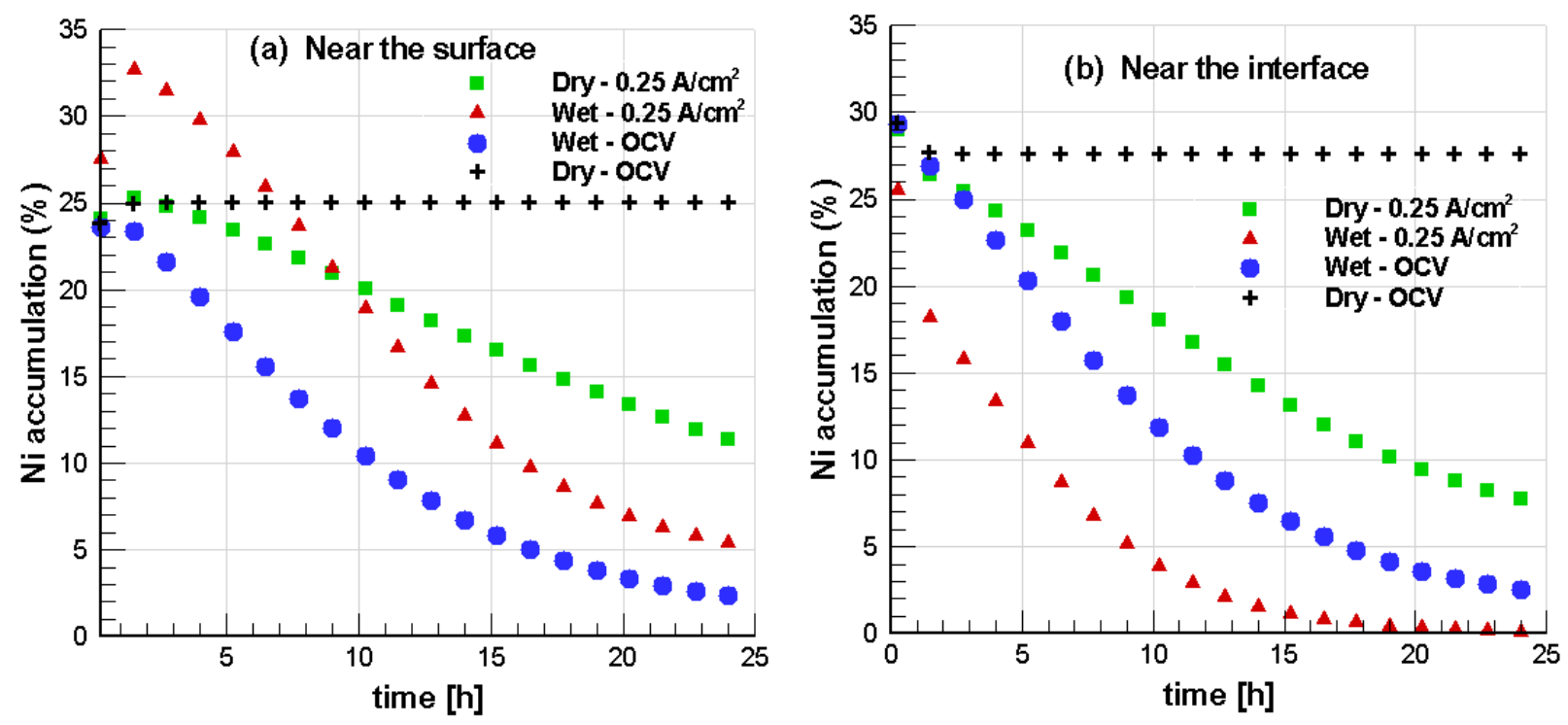

Figure B.12: Comparison of the sum of $\mathrm{Ni}$ accumulation under wet $\left(25 \% \mathrm{H}_{2} \mathrm{O}\right)$ at $\mathrm{OCV}$ and $0.25 \mathrm{~cm}^{2}$ current load, and dry at $0.25 \mathrm{~cm}^{2}$ current load: (a) Region I (b) Region II

In Figure B. 12a, it is seen that the Ni accumulation of region I under $0.25 \mathrm{~A} / \mathrm{cm}^{2}$ current density and with large $\mathrm{H}_{2} \mathrm{O}$ concentration ( $25 \%$ ) increases from its initial stage up to 5 hours of phosphine exposure. However, it decreases rapidly afterwards. This behavior can be explained by the effect 
of current load and large steam concentration due to the functional relation of drift velocity with moisture content and current density in electro-migration model, namely the current load and steam concentration facilitated the $\mathrm{Ni}$ migration towards region $\mathrm{I}$. The $\mathrm{Ni}$ consumption and migration is shown in Figure B. 12b. As it is seen in Figure B. 12b, Ni depletion under large steam concentration at OCV and wet-current conditions is much severe than that of dry-current condition.
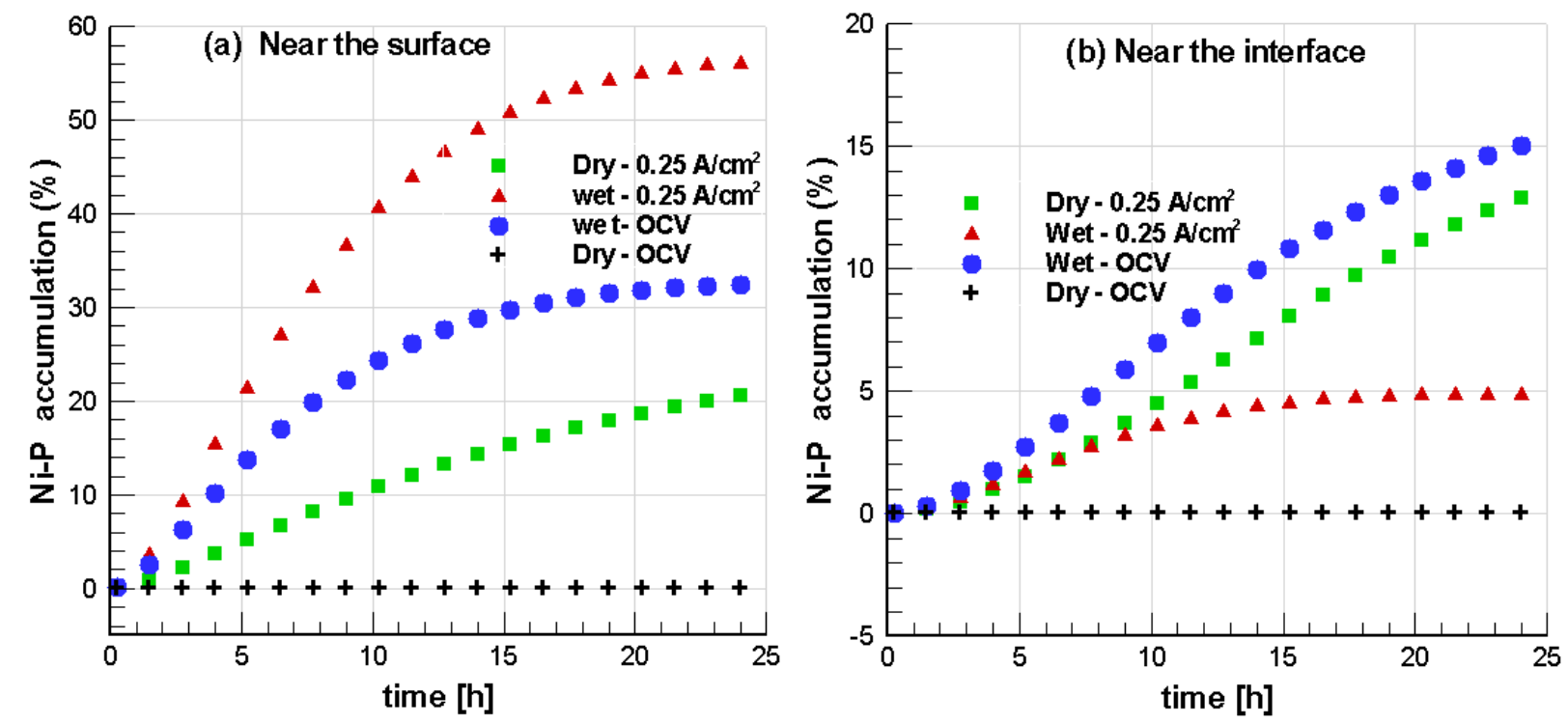

Figure B.13: Comparison of the sum of Ni-P (secondary phases) accumulation under (25\% $\mathrm{H}_{2} \mathrm{O}$ )at OCV and $0.25 \mathrm{~cm}^{2}$ current load, and dry at $0.25 \mathrm{~cm}^{2}$ current load: (a) Region I, (b) Region II 

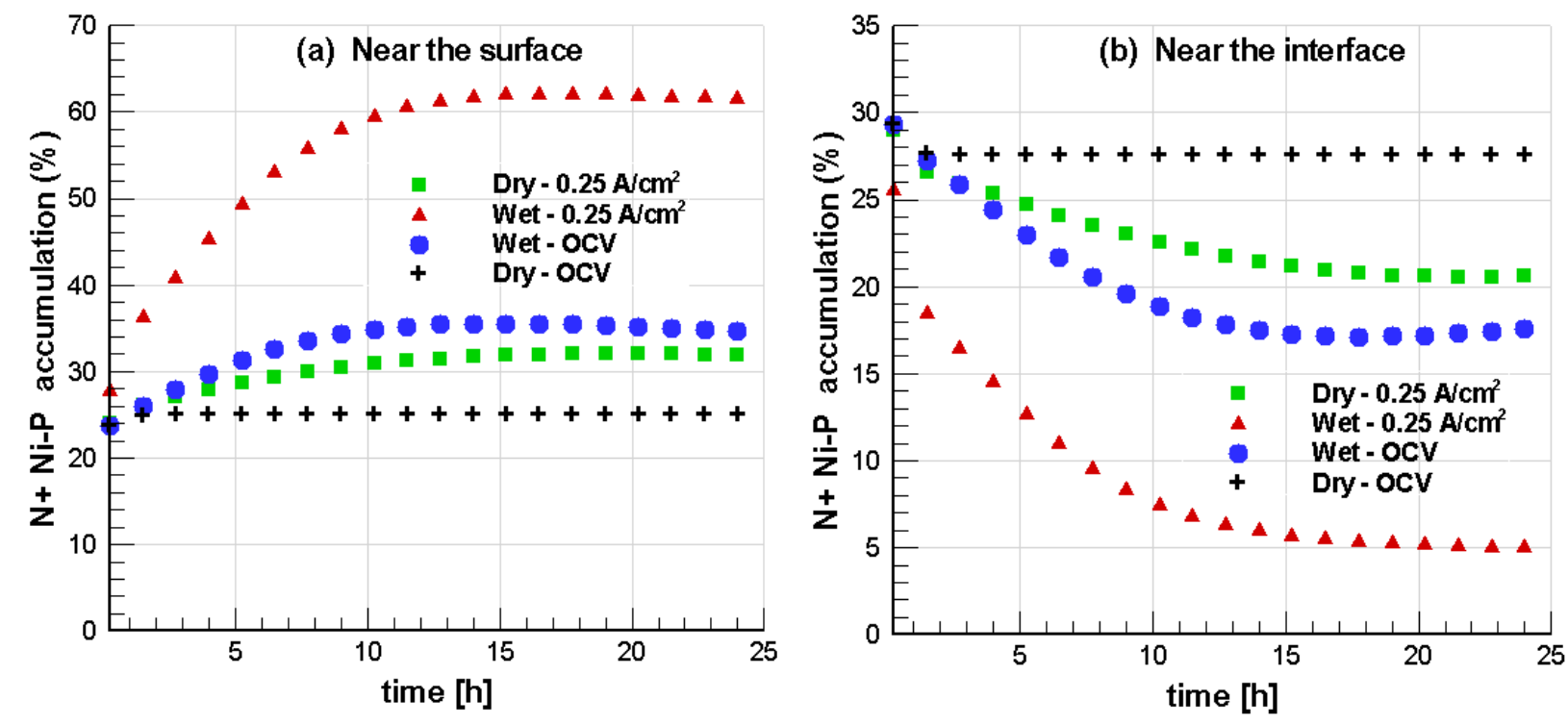

Figure B.14: Comparison of the sum $\mathrm{Ni}+\mathrm{Ni}-\mathrm{P}$ (secondary phases) accumulation under wet $\left(25 \% \mathrm{H}_{2} \mathrm{O}\right.$ ) at $\mathrm{OCV}$ and $0.25 \mathrm{~cm}^{2}$ current load, and dry at $0.25 \mathrm{~cm}^{2}$ current load: (a) Region I,

(b) Region II

This is due to influence of the large steam concentration on Ni-P formation in that region (Figure B. 13b). At $\mathrm{OCV}\left(25 \% \mathrm{H}_{2} \mathrm{O}\right)$ and $\operatorname{dry}\left(0.25 \mathrm{~A} / \mathrm{cm}^{2}\right)$ condition, the amount of $\mathrm{Ni}$ did not increase in region I from its initial stage because electro-migration does not have any effect on OCV condition and the magnitude of electro-migration is low in dry condition. In Figure B. 13a, Ni-P compound accumulation is shown. Ni-P compound source term is a function of available $\mathrm{Ni}$, steam and phosphine coverage. It is not directly a function of current load in our model. However, it is linked with Ni-P phases via amount of elemental Ni. Therefore, as it can be revealed in Figure B. 13a, under large steam concentration and loading current, Ni-P formation reached its maximum value. In contrast, at $\mathrm{OCV}\left(25 \% \mathrm{H}_{2} \mathrm{O}\right)$ and $\operatorname{dry}\left(0.25 \mathrm{~A} / \mathrm{cm}^{2}\right)$ condition, the amount of $\mathrm{Ni}-\mathrm{P}$ is smaller than wet condition with current. In other words, large steam concentration influences Ni-P formation more than current load. In region II, Ni-P phase formation at wet-OCV and dry condition with 
current increase faster than that of wet condition. This behavior is due to the severe elemental Ni migration from region II.
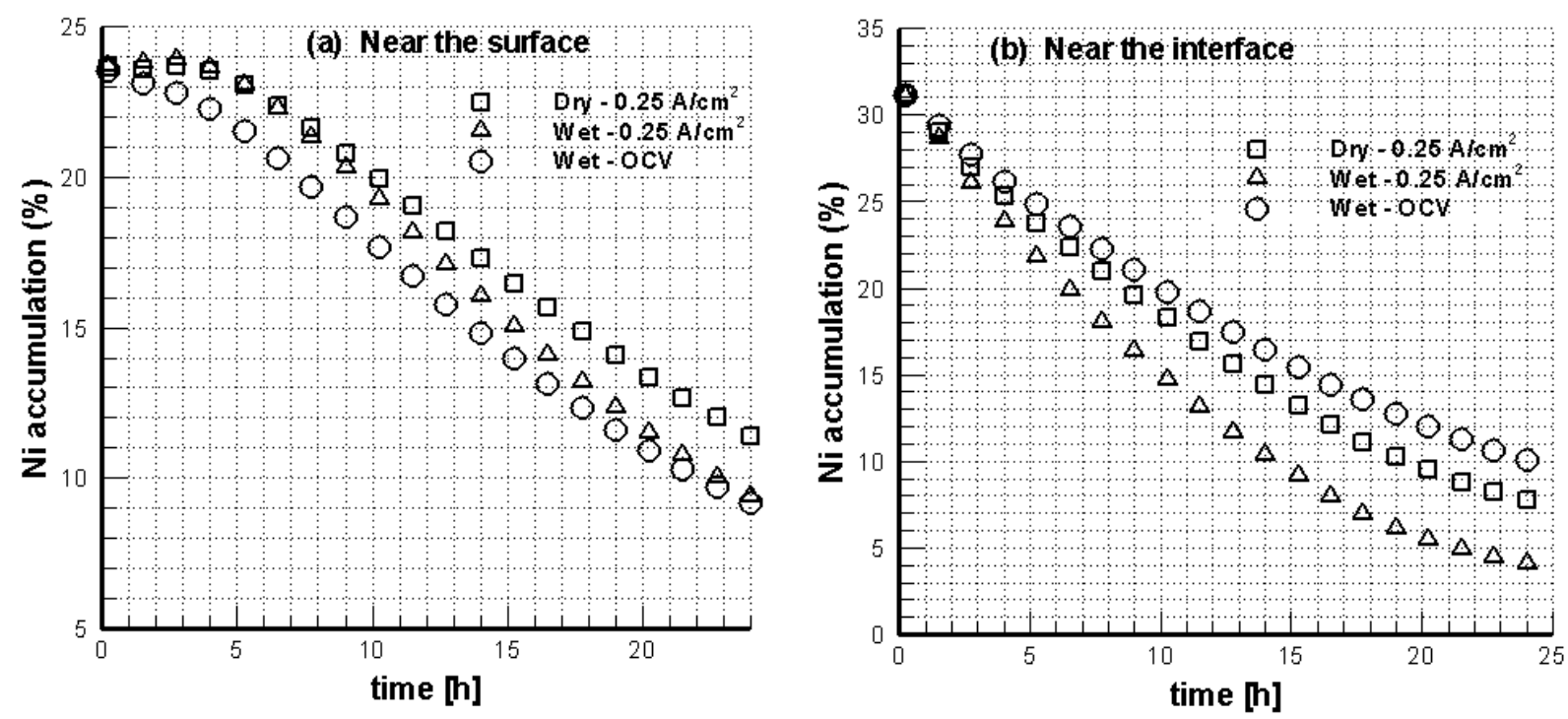

Figure B.15: Comparison of the sum of $\mathrm{Ni}$ accumulation under wet $\left(3 \% \mathrm{H}_{2} \mathrm{O}\right)$ at $\mathrm{OCV}$ and $0.25 \mathrm{~cm}^{2}$ current load, and dry at $0.25 \mathrm{~cm}^{2}$ current load: (a) Region I, (b) Region II
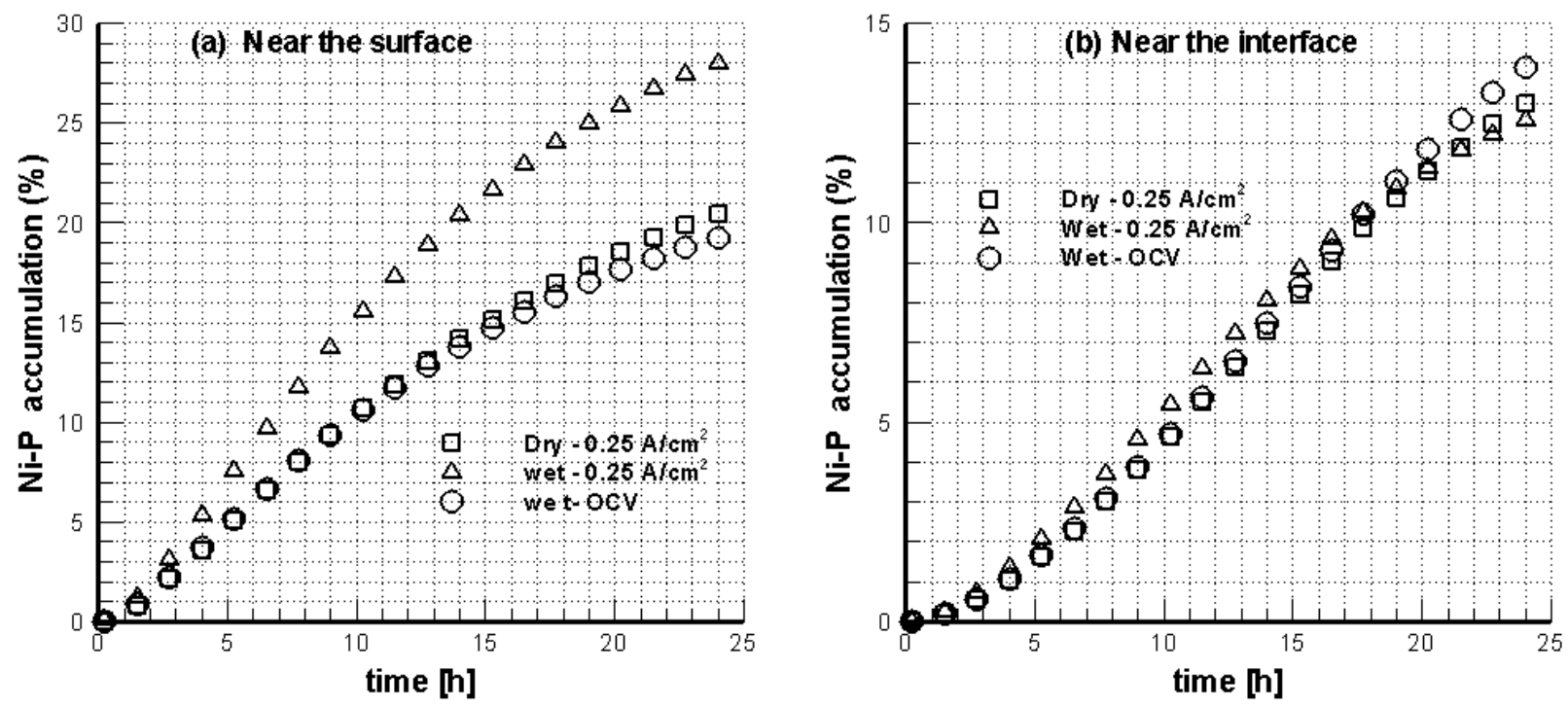

Figure B.16: Comparison of the sum of Ni-P accumulation under wet $\left(3 \% \mathrm{H}_{2} \mathrm{O}\right)$ at $\mathrm{OCV}$ and $0.25 \mathrm{~cm}^{2}$ current load, and dry $0.25 \mathrm{~cm}^{2}$ current load: (a) Region I, (b) Region II 
We quantify the Ni migration phenomena as the total available $\mathrm{Ni}$, elemental $\mathrm{Ni}$ and $\mathrm{Ni}$ present in Ni-P compounds. The amount of Ni+Ni-P compound in region I and II are depicted in Figure B. $14 \mathrm{a} \& \mathrm{~b}$. The $\mathrm{Ni}+\mathrm{Ni}-\mathrm{P}$ is observed to increase with the time of anode exposed to $\mathrm{PH}_{3}$. The $\mathrm{Ni}+\mathrm{Ni}-$ $\mathrm{P}$ compound of the anode operated at current density of $0.25 \mathrm{~A} / \mathrm{cm}^{2}$ shows faster increase than that without current load and without steam concentration. This illustrates that electric current and large steam concentration promote $\mathrm{Ni}+\mathrm{Ni}-\mathrm{P}$ accumulation in region I. In contrast, in region II, The $\mathrm{Ni}+\mathrm{Ni}-\mathrm{P}$ is observed to decrease with the degradation time. The $\mathrm{Ni}+\mathrm{Ni}-\mathrm{P}$ compound of the anode operated at current density of $0.25 \mathrm{~A} / \mathrm{cm}^{2}$ shows faster migration than that without current load and without steam concentration. In other words, the large steam concentration effect is larger than that of current load.
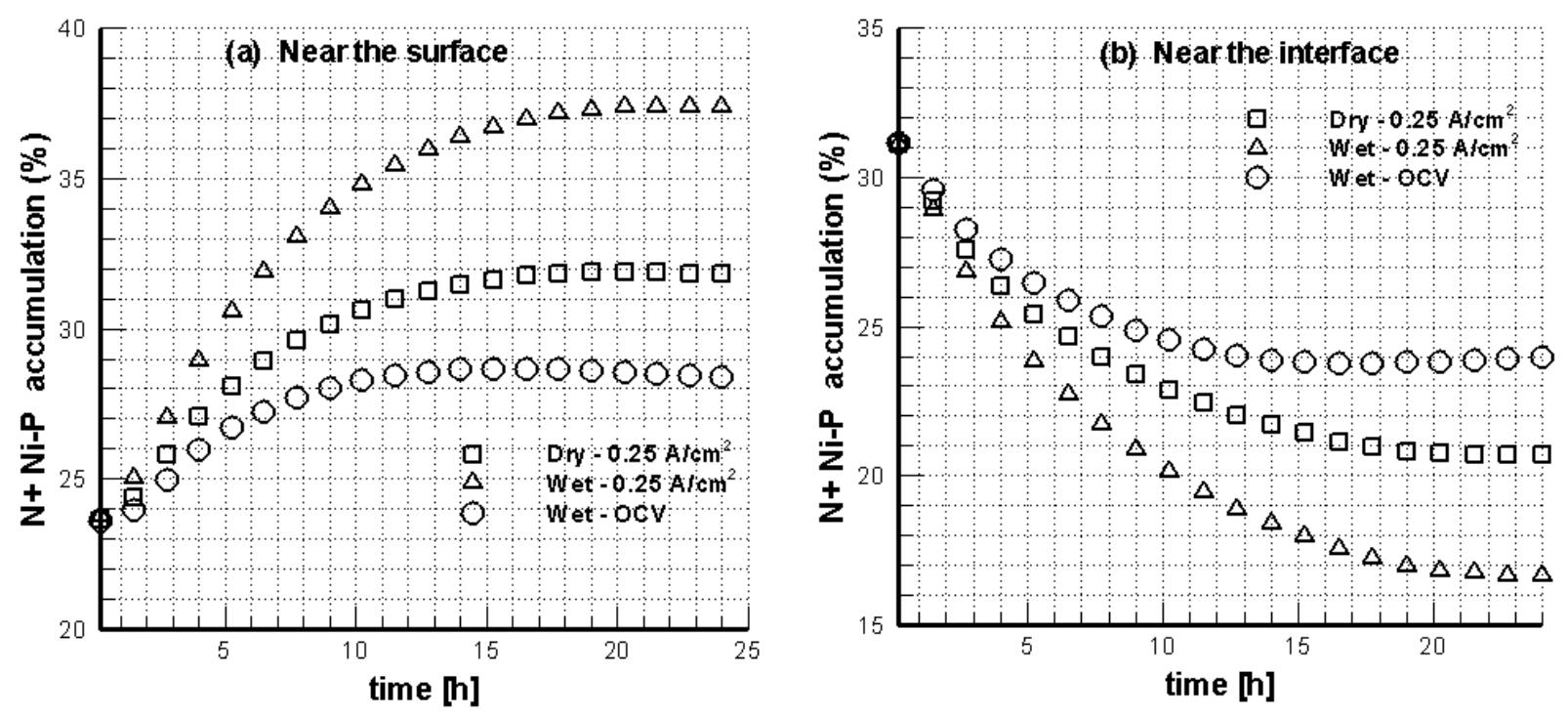

Figure B.17: Comparison of the sum $\mathrm{Ni}+\mathrm{Ni}-\mathrm{P}$ accumulation under wet $\left(3 \% \mathrm{H}_{2} \mathrm{O}\right)$ at $\mathrm{OCV}$ and $0.25 \mathrm{~cm}^{2}$ current load, and dry $0.25 \mathrm{~cm}^{2}$ current load: (a) Region I, (b) Region II Additional simulations were performed for low steam concentration. To compare the influences of current and low steam concentration and determine which factor is dominant in elemental redistribution of SOFC anode exposed to phosphine. In Figure B. 15-17a\&b, the influences of low 
steam and $0.25 \mathrm{~A} / \mathrm{cm}^{2}$ current density is shown for $\mathrm{Ni}, \mathrm{Ni}-\mathrm{P}$ phases, and $\mathrm{Ni}+\mathrm{Ni}+\mathrm{P}$ in region $\mathrm{I}$ and region II. It is seen from the results, the effect of the steam and current is still significant on elemental redistribution of SOFC anode. However, it can be concluded that, the effect of current and steam is almost at the same level for the Ni elemental accumulation. Even though the current seems to be dominant over the phosphine exposure time on $\mathrm{Ni}+\mathrm{Ni}-\mathrm{P}$ accumulation in region $\mathrm{I}$.

In Figure B. 18, Ni-P accumulation is illustrated. Experimental observation by Celik et al. [20] for Ni-P compound layer thicknesses on the anode surface after $\mathrm{PH}_{3}$ exposure at different current conditions were similar to that of the results shown in Figure B. 18. The secondary phases layer thickness (from experimental results by Celik et al. [20]) and Ni-P accumulation (in the current study) at current density of $0.25 \mathrm{~A} / \mathrm{cm}^{2}$ shows faster increase than that without current load. This observation indicates that the simulations and the experimental results are in good agreement.

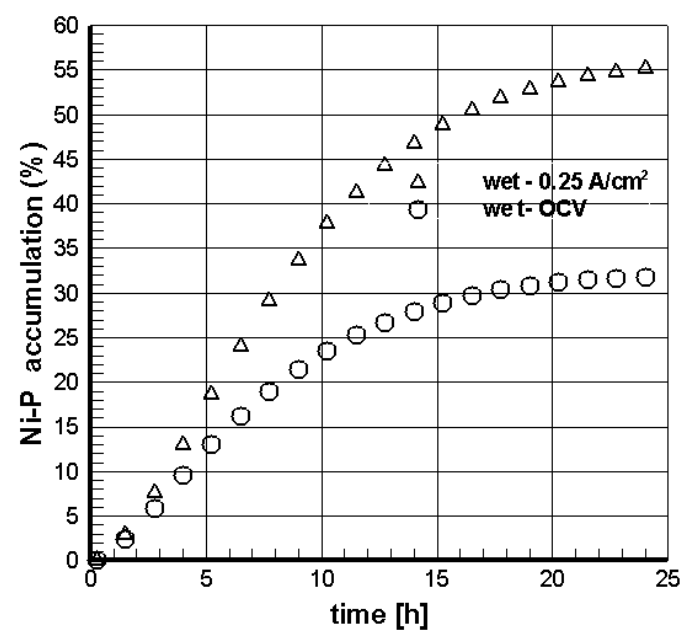

Figure B.18: The sum of Ni-P (secondary phases) accumulation under $\left(25 \% \mathrm{H}_{2} \mathrm{O}\right)$ at OCV and $0.25 \mathrm{~cm}^{2}$ current load, and dry at $0.25 \mathrm{~cm}^{2}$ current load in region I. 


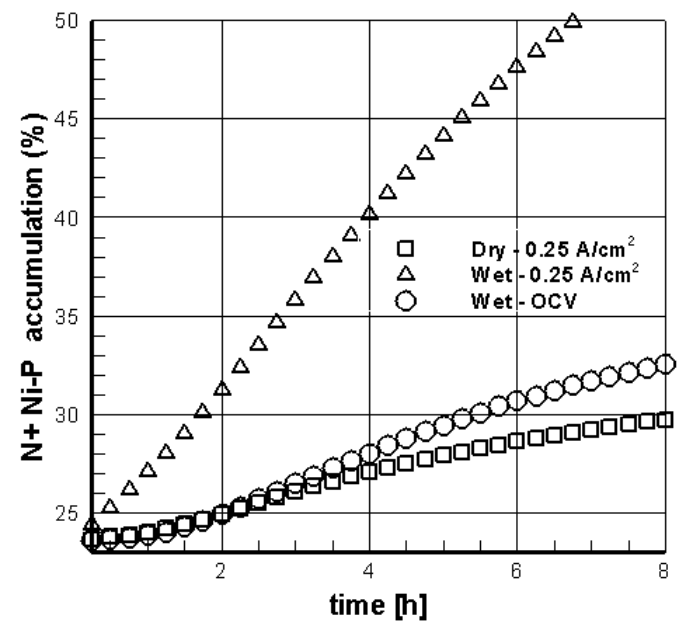

Figure B.19: The sum $\mathrm{Ni}+\mathrm{Ni}-\mathrm{P}$ (secondary phases) accumulation under wet $\left(25 \% \mathrm{H}_{2} \mathrm{O}\right)$ at OCV and $0.25 \mathrm{~cm}^{2}$ current load, and dry at $0.25 \mathrm{~cm}^{2}$ current load in Region I

In addition, the polarization resistance increase during the phosphine degradation was investigated by Celik. et al [20]. Polarization resistance is composed of mass transfer losses and activation losses. Mass transfer losses are due to the fuel diffusion limitation at triple phase boundaries. With the formation of secondary phases the diffusion of the fuel decreases during the phosphine exposure. In addition, secondary phases deactivate the available Ni sites which are related to the activation losses. Furthermore, elemental Ni migration and diffusion from active layer, where electrochemical reaction occurs, accelerate the activation losses. Therefore, the polarization resistance measured by Celik et al. [20]. could be proportional to the total $\mathrm{Ni}$ migration ( $\mathrm{Ni}$ elemental transport and Ni-P phases formation). In this context, the results that are obtained from simulation show similar behaviors with the experimental results (Figure B. 19). 


\section{B.5 Conclusions}

A phenomenological transport model for nickel migration in SOFC's exposed to low levels of phosphine is formulated based on mechanisms of electro-migration, diffusion and formation of secondary phases which are most likely responsible for Ni migration. This model is integrated with an in-house one-dimensional code for predicting SOFC anode degradation due to fuel impurities. The degradation model parameters were obtained as described in our previous work [46]. The over-all model is used to investigate the influence of current load and humidity in the elemental redistribution of SOFC anode exposed phosphine. Simulations were performed under different operating conditions. Results show that the Ni+Ni-P accumulation is much severe under high steam concentrations $\left(25 \% \mathrm{H}_{2} \mathrm{O}\right)$ than that of current load $\left(0.25 \mathrm{~A} / \mathrm{cm}^{2}\right)$. In contrast, under low steam concentrations $\left(3 \% \mathrm{H}_{2} \mathrm{O}\right)$, the influences of both steam and current load are of the same order of magnitude. Under high steam concentrations the influence of current load is higher than that of low steam concentration. This is due to the functional relation of drift velocity with steam concentration. Results also show that the steam concentration affects the formation of secondary phases and the electro-migration. Good agreement was achieved between model and available experimental data by Celik et al. 